Maja Pech

\title{
Local Methods for Relational Structures and their Weak Krasner Algebras
}

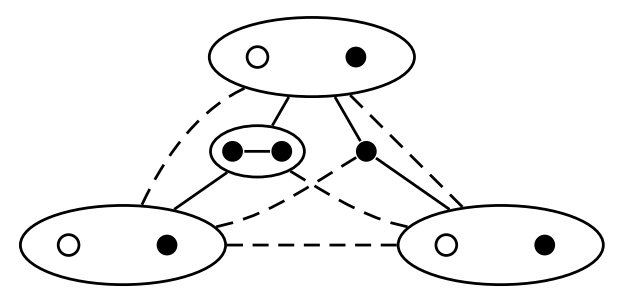

Novi Sad, 2009 



\section{Foreword}

It was a long journey, and at the end of the day it is time to sum up the impressions. The work on this thesis began in the spring 2005 during my stay at the Institute of Algebra in Dresden, Germany, in the frame of the programme GK 334: Specification of discrete processes and systems of processes by operational models and logics, and it continued at the same place in the next school year, thanks to the financial support from DAAD. My host and scientific father there was Professor Reinhard Pöschel, whom I owe my deepest gratitude for the influence that he made on this work, with many discussions, suggestions and useful ideas. The Institute of Algebra in Dresden was always a place where I was welcome, and I use this opportunity to thank all its members on scientific collaboration and many reinvitations to visit them and present my current work. There is no place like home, and all these years for me it was the Department of Mathematics and Informatics in Novi Sad, which provide me the opportunity to interrupt my teaching assistant work from time to time and to make many scientific visits abroad. One of them, that was of the great importance for this thesis, I made in the summer 2008. During two months at the Department of Mathematics at the Ben-Gurion University, Be'er Sheva, Israel, my host was Professor Mikhail Klin, whom I thank on several very interesting discussions and directions for the future work. Despite to many changes of workplaces and work conditions, one factor has stayed stable. Professor Dragan Mašulović is my supervisor since my very first scientific step, and I thank him on all advices, support and critics that improved my work.

A few people made all this possible, although they had nothing to do with the scientific part of this thesis. My mother-in-law was on many occasions taking the role of the Hotel Mamma, allowing me to concentrate on mathematics and to forget about everyday housework. Without any doubt, a great role played the quartet of dear people, my parents and my grandparents on my mother's side. They gave me all their love, support and the opportunity to freely choose my way in life. As a small sign of my gratitude, this thesis is dedicated to the memory of them.

My last and the greatest thanks go to my husband, for being both my retreat and the first line of the battle during the fight to obtain the results that are written on the pages that follow.

Novi Sad, March 2009

Maja Pech 



\section{Contents}

Introduction vii

Preliminaries

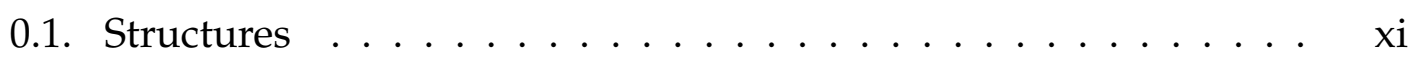

0.2. Logics and model theory . . . . . . . . . . . . . xii

0.3. Galois connections . . . . . . . . . . . . . xvii

0.4. Clone theory . . . . . . . . . . . . . . . . xviii

1. Local methods for relational structures 1

1.1. Locality Principle . . . . . . . . . . . . . . . . . . 2

1.2. Local methods for Rosenberg relations . . . . . . . . . . . . . 5

1.3. Applications of local methods (Examples) . . . . . . . . . . 17

2. Endolocal relational structures 21

2.1. Towards the notion of endolocality . . . . . . . . . . . . 21

2.2. Endolocality . . . . . . . . . . . . . . . 25

2.3. Model-theoretical characterizations of endolocality . . . . . . 32

2.4. Main Theorem . . . . . . . . . . . . . . . . 41

2.5. Endolocality and weak Krasner algebras . . . . . . . . . . . . . 43

3. Homomorphism-homogeneous relational structures 53

3.1. Minimal witness criterion . . . . . . . . . . . . . 53

3.2. Binary homomorphism-homogeneous relations . . . . . . . . 60

A. Small homomorphism-homogeneous tolerance relations 83

A.1. Relations on 4 elements . . . . . . . . . . . . . . . . . . 84

A.2. Relations on 5 elements . . . . . . . . . . . . . . . . . . 84

A.3. Relations on 6 elements . . . . . . . . . . . . . . . . . . 84

A.4. Relations on 7 elements . . . . . . . . . . . . . . . . . . . . 85

A.5. Relations on 8 elements . . . . . . . . . . . . . . 85

A.6. Relations on 9 elements . . . . . . . . . . . . . . . . 87

B. Sažetak 93

$\begin{array}{ll}\text { Bibliography } & 101\end{array}$ 



\section{Introduction}

Clone theory is a modern mathematical discipline that studies functional and relational algebras. The ideas that are built in its foundations are coming from several sources. One of them is the elementary theory of $0 / 1$ functions, introduced by George Boole in 1847 by proposing to handle propositional connectives as discrete truth-functions (cf. [45]). This led to the study of closed classes of truth functions in two-valued and multivalued logics. The other important source is the classical Galois theory. Inspired by Felix Klein's Erlangen program from 1872, it evolved into the general Galois theory for functions and relations. The pioneers in this field were Marc Krasner (cf. [21, 47]) and José Sebastião e Silva (cf. [50, 48]). Their research has to be seen in the context with the struggle of the early twentieth-century mathematics to come up with an abstract concept of structures, which ultimately led to the development of general algebra and model theory. The theory of functional algebras and the general Galois theory for functions and relation developed gradually into what is today known as clone theory (see e.g. [19, 20],[15, 2, 52, 35]).

The main problem of clone theory is to describe the clone lattice for the given basic set. The first major result in this area was obtained by Emil Leon Post in [42, 43], where he was describing the clone lattice on the two-element basic set. So far, this is the only completely studied clone lattice. Already on the three-element basic set the full structure of the lattice is still unknown. Since the complete description of the clone lattices in general is considered to be hopeless, the focus of research goes to the study of its substructures and its approximations.

One way of approximating the clone lattice is to look at the $k$-ary parts of the clones and to describe their mutual inclusions. One of the starting points of this research was to study their unary parts, i.e. transformation monoids. The first class of relations whose endomorphism monoids and their mutual relationships were studied was the class of Rosenberg relations (cf. [36, 30, 34, 38]). The choice was not surprising, since they describe maximal clones. It turned out that the unary parts of maximal clones are almost always incomparable, except for a few classes of relations (e.g. central, $h$-regular relations). However, ad hoc methods that were used in this study were not able to bring a breakthrough in some of the cases where inclusion between the endomorphism monoids tend to exist. This raised a need to find new methods for solving problems of the mentioned kind, which led to the development of local methods. They appeared as a tool for the first time in [37], where they were used in the study of the poset of endomorphism monoids of central relations. After that, they were successfully applied to a few more classes of relations in [33]. In [29], a surprising relationship between local methods and homomorphism-homogeneous relations was pointed out. 
In particular, whenever we deal with a homomorphism-homogeneous relation, local methods can be used. This suggested to study more deeply the connection between homomorphism-homogeneity and the goals of our research.

The concept of homomorphism-homogeneity of relational structures was introduced by Peter Cameron and Jaroslav Nešetřil in [5], as an interesting and natural generalization of the classical notion of homogeneity. There are many combinatorial results on the subject of the classification of homogeneous relational structures (cf. [14, 24, 49, 23, 7, 3, 16]). This motivated Cameron and Nešetřil to ask and study similar questions about homomorphism-homogeneity of graphs, and binary relations in general. Meanwhile, the first results on this subject appeared. The strict orders that are homomorphism-homogeneous were characterized by Cameron and Debbie Lockett in [4] and the partial orders with this property were characterized by Dragan Mašulović in [28]. After that, the attention was turned back to graphs which led to the characterizations of homomorphismhomogeneous finite tournaments with loops in [18].

The study of the classical homogeneity is an area of high interest because of its connections to the quantifier elimination property, the property of theories to be $\omega$-categorical, etc. Moreover, there is a well-developed theory about constructions of homogeneous structures due to the work of Roland Fraïssé (cf. [10, 11]). On the other hand, the study of homomorphism-homogeneous structures was, until now, more a combinatorial problem driven by curiosity.

In connection with previously presented facts, we can now formulate the goals of this thesis. First of all, we want to make local methods available as a tool to study the unary parts of clones in general. Secondly, it is our aim to develop a link between homomorphism-homogeneous relational structures and local methods. Finally, our third goal is to develop a systematic theory for the classification of homomorphism-homogeneous structures.

The thesis starts with a chapter about local methods. There we define the Locality Principle and introduce local methods. In the sequel we show how they can be applied in the study of the structure of weak Krasner algebras and we make local methods available for all classes of Rosenberg relations. The applications of local methods are demonstrated by a couple of examples.

In Chapter 2 we systematize the use of the Locality Principle and create a connection with logics. The notion of $k$-endolocality is introduced, and by this the relational structures are put into a hierarchy. In the next step, $k$-endolocal relational structures are studied from the point of view of model theory. Modeltheoretical notions that are playing a role in the systematic study of homogeneous structures are adapted to make them useful for the study of $k$-endolocal relational structures. For instance, the notion of oligomorphic structures is adapted to the notion of weakly oligomorphic structures. Also, the corresponding notion of weak homogeneity is the one-point extension property, while the elimination sets are adopted to the positive existential elimination sets. This chapter culminates with the Main Theorem that describes the mutual connections between all these notions. This creates a link between homomorphism-homogeneous relational 
structures and model-theoretical notions, and it opens the ways for a systematic study of homomorphism-homogeneous structures using both model-theoretical and algebraic methods. The Main Theorem has also important consequences for clone theory. For instance, weakly oligomorphic weak Krasner algebras on a countable basic set are always locally closed. This generalizes a part of the main theorem of clone theory (cf. [40, Hauptsatz 2.1.3.(ii)]) to weakly oligomorphic relational structures. On the other hand, our Main Theorem enables us to use local methods for all weak Krasner algebras on finite basic sets.

Chapter 3 uses the theory that was developed in Chapter 2 in order to derive a systematic method for characterizing homomorphism-homogeneous relational structures. The key notion in this part of the thesis is the notion of minimal witnesses. These are special subconfigurations that are forbidden in all relational structures that have the one-point extension property. If for a given class of relational structures all minimal witnesses can be found up to isomorphism, then this, in a sense, characterizes all structures from the given class that have the one-point extension property. We proceed by describing all minimal witnesses up to isomorphism for the class of antisymmetric transitive binary relations (i.e. for the structures of the shape $(A, \varrho)$, where $\varrho$ is a binary antisymmetric transitive relation.). These results are used to characterize all transitive binary relations with one-point extension property. This generalizes the results by Cameron, Locket and Mašulović about strict orders and partial orders (cf. [4, 28]). The minimal witnesses for tolerance relations are also described in some way. There can appear every possible configuration as a witness. However, this is expected because, as it was shown in [46], the problem of deciding homomorphism-homogeneity for symmetric binary relations is co-NP complete. Yet, homomorphism-homogeneous tolerance relations over finite sets are relatively rare. In Appendix A, we list, up to isomorphism, all nontrivial homomorphism-homogeneous relations on up to 9 elements. 



\section{Preliminaries}

In this chapter we will fix the notions and notation from logics, model theory, clone theory and formal concept analysis that will be used throughout the thesis. The goal is not to give a comprehensive introduction into the respective fields. Rather, it is to make the exposition of this treatise mostly self-contained. Additional references will be given, where appropriate.

\subsection{Structures}

Structures represent one of the most fundamental notions in mathematics. The central object of the study in this thesis are relational structures, so we start by introducing them formally. We say that a set $R$ of relational symbols together with a mapping ar : $R \rightarrow \mathbb{N}$ that maps every symbol to its arity is a relational signature. For a given relational signature $R$ and a given basic set $A$, we define the notion of relational structure $\mathbf{A}$ over the signature $R$ (or, for short, $R$-structure) as an ordered pair $\left(A,\left(\varrho_{\mathbf{A}}\right)_{\varrho \in R}\right)$, where $\varrho_{\mathbf{A}}$ is a relation of arity $\operatorname{ar}(\varrho)$ over $A$ that interprets in $\mathbf{A}$ the relational symbol $\varrho \in R$. With $R^{(n)}$ we will denote the set of all relational symbols from $R$ of arity $n$. The elements of $\varrho_{\mathbf{A}}$ will be denoted by $\bar{a}, \bar{b}, \bar{c}, \ldots$ In general, if $\mathbf{A}$ is a relational structure, then with $A$ we will denote the basic set of $\mathbf{A}$. We call an element $\bar{x}=\left(x_{1}, \ldots, x_{\operatorname{ar}(\varrho)}\right)$ reflexive if there are $i \neq j$ such that $x_{i}=x_{j}$. Otherwise, we say that $\bar{x}$ is irreflexive. According to this, $\varrho_{\mathbf{A}}{ }^{\text {irr }}$ will represent the set of all irreflexive elements of the relation $\varrho_{\mathrm{A}}$. By $\Delta_{A}$ we denote the diagonal relation on $A$, i.e.

$$
\Delta_{A}:=\{(x, x) \mid x \in A\} .
$$

For a given relational signature $R$ and two relational structures $\mathbf{A}=\left(A,\left(\varrho_{\mathbf{A}}\right)_{\varrho \in R}\right)$ and $\mathbf{B}=\left(B,\left(\varrho_{\mathbf{B}}\right)_{\varrho \in R}\right)$, we call a mapping $f: A \rightarrow B$ a homomorphism if for every $\varrho \in R$ holds that from $\left(x_{i_{1}}, \ldots, x_{i_{\text {ar }(\rho)}}\right) \in \varrho_{\mathbf{A}}$, it follows that $\left(f\left(x_{i_{1}}\right), \ldots, f\left(x_{i_{\text {ar }(o)}}\right)\right) \in \varrho_{\mathbf{B}}$. Further, a mapping $f: A \rightarrow B$ is called a full homomorphism if for all $\varrho \in R$ and all $\left(x_{i_{1}}, \ldots, x_{i_{\text {ar }(\varrho)}}\right) \in A^{\operatorname{ar}(\varrho)}$ we have

$$
\left(x_{i_{1}}, \ldots, x_{i_{\operatorname{ar}(\varrho)}}\right) \in \varrho_{\mathbf{A}} \Leftrightarrow\left(f\left(x_{i_{1}}\right), \ldots, f\left(x_{i_{\operatorname{ar}(\varrho)}}\right)\right) \in \varrho_{\mathbf{B}} .
$$

Injective homomorphisms are called monomorphisms, while surjective homomorphism are called epimorphisms. A homomorphism $f: \mathbf{A} \rightarrow \mathbf{A}$ will be called an endomorphism of $\mathbf{A}$. Full monomorphisms are called embeddings and bijective embeddings are called isomorphisms. A local homomorphism of $A$ is a homomorphism from a finite substructure of $\mathbf{A}$ to $\mathbf{A}$. 
We say that $\mathbf{B}$ is a substructure of $\mathbf{A}$ (and write $\mathbf{B} \leq \mathbf{A}$ ) if $B \subseteq A$ and the inclusion map $\iota: B \rightarrow A$ is an embedding. Substructures are fully determined by their basic sets. If $\mathbf{B} \leq \mathbf{A}$ and $C \subseteq A$, then $\mathbf{B} \cup C$ shall denote the substructure of $\mathbf{A}$ with the basic set $B \cup C$. A retraction is an epimorphism with a right inverse. In particular, an epimorphism $r: \mathbf{A} \rightarrow \mathbf{B}$ is a retraction if there exists an embedding $i: \mathbf{B} \hookrightarrow \mathbf{A}$ such that $r \circ i$ is the identity mapping on $B$. In this case $\mathbf{B}$ is called $\mathbf{a}$ retract of $\mathbf{A}$. We say that $\mathbf{A}$ is a full homomorphic preimage of $\mathbf{B}$ if there exists a full homomorphism $f: \mathbf{A} \rightarrow \mathbf{B}$.

\subsection{Logics and model theory}

Throughout the thesis we will use notions from logics and model theory. In this section we fix only those notions and notation from model theory that will be needed in the sequel. For a detailed treatment we refer to [17] and [6].

0.2.1. Syntax and semantics. Let $R$ be a relational signature and let $X=\left\{x_{i} \mid\right.$ $i \in \mathbb{N}\}$ be a set of distinct variable symbols. An atomic formula is a formula of the shape $\left(x_{i_{1}}=x_{i_{2}}\right)$ or $\varrho\left(x_{i_{1}}, \ldots, x_{i_{\operatorname{ar}(\varrho)}}\right)$, where $\varrho \in R$ and $x_{i_{1}}, \ldots, x_{i_{\text {ar }(\rho)}} \in X$. In order to obtain more complex formulae from already defined ones we introduce the following rules:

AND If $\Psi$ is a finite set of formulae, then $\bigwedge \Psi$ is a formula.

OR If $\Psi$ is a finite set of formulae, then $\bigvee \Psi$ is a formula.

EXISTS If $\psi$ is a formula and $x \in X$, then $(\exists x) \psi$ is a formula.

The set of primitive positive formulae is the smallest set of words that contains all atomic formulae and that is closed with respect to rules AND and EXISTS. We denote it by $\Phi(\exists, \wedge,=, R)$. The set of positive existential formulae is the smallest set of words that contains all atomic formulae and that is closed with respect to rules AND, OR and EXISTS. We denote it by $\Phi(\exists, \wedge, \vee,=, R)$. For a certain kind of formulae we introduce a special notation:

\begin{tabular}{|c|c|}
\hline Formula & Abbreviation \\
\hline$\bigwedge \emptyset$ & $\mathrm{F}$ \\
\hline$\bigvee \emptyset$ & $\mathrm{T}$ \\
\hline$\bigwedge\left\{\psi_{i} \mid i \in I\right\}$ & $\bigwedge_{i \in I} \psi_{i}$ \\
\hline$\bigvee\left\{\psi_{i} \mid i \in I\right\}$ & $\bigvee_{i \in I} \psi_{i}$ \\
\hline$\bigwedge\left\{\psi_{i_{1}}, \psi_{i_{2}}\right\}$ & $\psi_{i_{1}} \wedge \psi_{i_{2}}$ \\
\hline$\bigvee\left\{\psi_{i_{1}}, \psi_{i_{2}}\right\}$ & $\psi_{i_{1}} \vee \psi_{i_{2}}$ \\
\hline
\end{tabular}

A variable $x$ appears freely in formula $\psi$ (i.e. it is a free variable) if it appears in $\psi$ unquantified. To make this more precise, we define a function free : $\Phi(\exists, \wedge, \vee,=$ $, R) \rightarrow \mathcal{P}(X)$ that assigns to each formula the set of variable symbols that appear freely in it. We proceed by structural induction: 
- If $\psi$ is of the shape $\left(x_{i}=x_{j}\right)$, then we define free $(\psi):=\left\{x_{i}, x_{j}\right\}$.

- If $\psi$ is of the shape $\varrho\left(x_{i_{1}}, \ldots, x_{i_{\operatorname{ar}(\varrho)}}\right)$ then we define free $(\psi):=\left\{x_{i_{1}}, \ldots, x_{i_{\operatorname{ar}(\varrho)}}\right\}$.

- If we are given a finite set $\Psi$ of formulae then we define free $(\bigwedge \Psi)=$ free $(\bigvee \Psi):=\bigcup_{\psi \in \Psi} \operatorname{free}(\psi)$.

- If we are given a formula $\psi$, then free $((\exists x) \psi):=$ free $(\psi) \backslash\{x\}$.

We define $\Phi_{m}(\exists, \wedge, \vee,=, R)$ as the set of all formulae $\varphi \in \Phi(\exists, \wedge, \vee,=, R)$ such that free $(\varphi) \subseteq\left\{x_{1}, \ldots, x_{m}\right\}$. The set $\Phi_{m}(\exists, \wedge,=, R)$ is defined analogously.

Let $\mathbf{A}=\left(A,\left(\varrho_{A}\right)_{\varrho \in R}\right)$ be a relational structure. We define what means that a structure A models a formula $\psi$ with respect to the valuation $v: X \rightarrow A$ :

$$
\begin{array}{lll}
\mathbf{A} \models_{v} \varrho\left(x_{i_{1}}, \ldots, x_{\operatorname{ar}(\varrho)}\right) & : \Leftrightarrow\left(v\left(x_{i_{1}}\right), \ldots, v\left(x_{\operatorname{ar}(\varrho)}\right)\right) \in \varrho_{\mathbf{A}} \\
\mathbf{A} \models_{v}\left(x_{i}=x_{j}\right) & : \Leftrightarrow v\left(x_{i}\right)=v\left(x_{j}\right) \\
\mathbf{A} \models_{v} \bigwedge \Psi & : \Leftrightarrow \text { For all } \psi \in P s i: \quad \mathbf{A} \models_{v} \psi \\
\mathbf{A} \models_{v} \bigvee \Psi & : \Leftrightarrow \text { There exists a } \psi \in P s i: \quad \mathbf{A} \models_{v} \psi \\
\mathbf{A} \models_{v}(\exists x) \psi & : \Leftrightarrow \text { There exists an } a \in A \text { such that } \mathbf{A} \models_{v_{(x / a)}} \psi \\
& & \text { where } v_{(x / a)}: X \rightarrow A \text { is given by } \\
& v_{(x / a)}(y)=\left\{\begin{aligned}
a, & \text { if } y=x \\
v(y), & \text { otherwise. }
\end{aligned}\right.
\end{array}
$$

Let $\varphi \in \Phi_{m}(\exists, \wedge, \vee,=, R)$ and let $\bar{a}=\left(a_{1}, \ldots, a_{m}\right) \in A^{m}$. We say that a tuple $\bar{a}$ satisfies the formula $\varphi$ and write $\bar{a} \vDash \varphi$ if there exists a valuation $v$ such that $v\left(x_{i}\right)=a_{i}$, for all $i \in\{1, \ldots, m\}$ and such that $\mathbf{A} \models_{v} \varphi$. The set of all tuples $\bar{a} \in A^{m}$ such that $\bar{a} \vDash \varphi$ we denote by $\varphi^{\mathbf{A}}$. Further, for a set $B \subseteq A^{m}$ of tuples we define

$$
\operatorname{pTh}_{\mathbf{A}}(B):=\left\{\varphi \in \Phi_{m}(\exists, \wedge, \vee,=, R) \mid \forall \bar{b} \in B: \bar{b} \vDash \varphi\right\} .
$$

For a tuple $\bar{b} \in A^{m}$, instead of $\operatorname{pTh}_{\mathbf{A}}(\{\bar{b}\})$, we write $\mathrm{pTh}_{\mathbf{A}}(\bar{b})$.

For formulae $\varphi$ and $\psi$ from $\Phi(\exists, \wedge, \vee,=, R)$ we say that they are equivalent if for every relational structure $\mathbf{A}$ over the signature $R$ and every valuation $v$ in $A$ holds $\mathbf{A} \models_{v} \varphi$ if and only if $\mathbf{A} \models_{v} \psi$. In this case we write $\varphi \equiv \psi$. Further, we say that $\varphi$ and $\psi$ from $\Phi(\exists, \wedge, \vee,=, R)$ are equivalent in the given structure $\mathbf{A}$ if for every valuation $v$ in $A$ holds $\mathbf{A} \models_{v} \varphi$ if and only if $\mathbf{A} \models_{v} \psi$.

It is a well-known fact that every primitive positive formula $\varphi$ is equivalent to a formula of the following shape:

$$
\varphi \equiv\left(\exists x_{i_{1}}\right)\left(\exists x_{i_{2}}\right) \ldots\left(\exists x_{i_{k}}\right) \bigwedge_{j \in J} \psi_{j}
$$

where $i_{1}, \ldots, i_{k} \in \mathbb{N}$ and $\psi_{j}, j \in J$, are atomic formulae. Such a formula is called a prenex normal form. Every positive existential formula $\varphi$ can be then written in the form:

$$
\varphi \equiv \bigvee_{i \in I} \varphi_{i}
$$


where $\varphi_{i}, i \in I$, are primitive positive formulae in prenex normal form. We call such a formula a normal form formula.

The quantifier depth of a primitive positive formula $\varphi$ in prenex normal form $\left(\exists x_{i_{1}}\right)\left(\exists x_{i_{2}}\right) \ldots\left(\exists x_{i_{k}}\right) \bigwedge_{j \in J} \psi_{j}$ is $k$. We denote it by $\mathrm{qd}(\varphi)=k$. The quantifier depth of a normal form formula $\varphi$ of the shape $\bigvee_{i \in I} \varphi_{i}$ is defined as $\operatorname{qd}(\varphi):=\max \left\{q \mathrm{~d}\left(\varphi_{i}\right) \mid\right.$ $i \in I\}$. The quantifier depth of any positive existential formula $\varphi$ is defined as the minimum of all quantifier depths of normal form formulae that are equivalent to $\varphi$. For any set $\Psi \subseteq \Phi(\exists, \wedge, \vee,=, R)$ we denote by $\Psi^{(k)}$ the set of all formulae with quantifier depth at most $k$ from $\Psi$.

\subsubsection{On the number of formulae in different fragments of logics.}

Proposition. Let $R$ be a finite relational signature. Then, up to equivalence, there are just finitely many formulae in $\Phi_{m}^{(0)}(\exists, \wedge, \vee,=, R)$.

Proof. Let $\varphi \in \Phi_{m}^{(0)}(\exists, \wedge, \vee,=, R)$. Then

$$
\varphi \equiv \bigvee_{i \in I} \bigwedge_{j \in J_{i}} \varphi_{i j}
$$

where $\varphi_{i j}$ are atomic formulae. In the next step, we will estimate the number of atomic formulae in $\Phi_{m}^{(0)}(\exists, \wedge, \vee,=, R)$.

Note that for every $\varphi_{i j}$ we have that either $\varphi_{i j} \equiv \varrho\left(x_{k_{1}}, \ldots, x_{k_{n}}\right)$, for $\varrho \in R^{(n)}, n \in \mathbb{N}$ and $x_{k_{1}}, \ldots, x_{k_{n}} \in\left\{x_{1}, \ldots, x_{m}\right\}$ or $\varphi_{i j} \equiv\left(x_{k_{1}}=x_{k_{2}}\right)$, for $x_{k_{1}}, x_{k_{2}} \in\left\{x_{1}, \ldots, x_{m}\right\}$. Since $R$ is finite, there are finitely many relational symbols $\varrho$ and, since $\left\{x_{1}, \ldots, x_{m}\right\}$ is finite, there are finitely many choices for the entries of tuples, so there are finitely many atomic formulae of the shape $\varrho\left(x_{k_{1}}, \ldots, x_{k_{n}}\right)$. The similar reasoning gives us that there are finitely many atomic formulae of the shape $\left(x_{k_{1}}=x_{k_{2}}\right)$, so there are all together finitely many atomic formulae.

Note further that every $\bigwedge_{j \in J_{i}} \varphi_{i j}$ is equivalent to the conjunction of a subset of atomic formulae, so $\left|J_{i}\right|$ is bounded by the number of atomic formulae, and therefore, there are, up to equivalence, finitely many conjunctions of atomic formulae.

Finally, every $\bigvee_{i \in I} \bigwedge_{j \in I_{i}} \varphi_{i j}$ is equivalent to the disjunction of a set of conjunctions of atomic formulae, so $|I|$ is bounded by the number of conjunctions of atomic formulae (up to equivalence), and by the same reasoning, there are finitely many formulae in $\Phi_{m}^{(0)}(\exists, \wedge, \vee,=, R)$.

Corollary. Let $R$ be a finite relational signature. Then, up to equivalence, there are finitely many formulae in $\Phi_{m}^{(k)}(\exists, \wedge, \vee,=, R)$.

Proof. Let $\varphi \in \Phi_{m}^{(k)}(\exists, \wedge, \vee,=, R)$ is of the shape

$$
\varphi \equiv\left(\exists x_{m+1}\right)\left(\exists x_{m+2}\right) \ldots\left(\exists x_{m+k}\right) \psi,
$$

where $\psi \in \Phi_{m+k}^{(0)}(\exists, \wedge, \vee,=, R)$. By the previous Proposition there are, up to equivalence, just finitely many formulae $\psi$. Then, up to equivalence, there are just finitely many formulae $\varphi$. 
0.2.3. Relational configurations. We will now introduce the notion of relational configurations and establish a correspondence between them and primitive positive formulae. Relational configurations will allow us to work with primitive positive formulae in a syntax-free and algebraic manner.

Definition 1. Let $\mathbf{D}=\left(D,\left(\varrho_{\mathbf{D}}\right)_{\varrho \in R}\right)$ be a relational structure over a finite set $D$. Further, let $\mathbf{C}$ be a substructure of $\mathbf{D}$, let $\theta \subseteq D^{2}$ and let $E:\{1, \ldots,|C|\} \rightarrow C$ be a fixed enumeration of elements of $C$. Then the quadruple $(\mathbf{C}, \mathbf{D}, E, \theta)$ is called $\mathbf{a}$ relational configuration over $R$.

We say that a tuple $\bar{a}=\left(a_{1}, \ldots, a_{|C|}\right) \in A^{|C|}$ satisfies $(\mathbf{C}, \mathbf{D}, E, \theta)$ in $\mathbf{A}$ and write $\bar{a} \|(\mathbf{C}, \mathbf{D}, E, \theta)$ if there exists a homomorphism $g: \mathbf{D} \rightarrow \mathbf{A}$ such that

(G1) $g: E(i) \mapsto a_{i}$, for $i=1, \ldots,|C|$ and

(G2) $\theta \subseteq \operatorname{ker} g$.

Proposition. Let $R$ be a relational signature and let $m \in \mathbb{N}$. Then the following are true:

1. For every $\varphi \in \Phi_{m}(\exists, \wedge,=, R)$ there exists a relational configuration $(\mathbf{C}, \mathbf{D}, E, \theta)$ over $R$ such that for all relational structures $\mathbf{A}=\left(A,\left(\varrho_{\mathbf{A}}\right)_{\varrho \in R}\right)$ and for all $\bar{a} \in A^{m}$

$$
\bar{a} \vDash \varphi \Leftrightarrow \bar{a} \Vdash(\mathbf{C}, \mathbf{D}, E, \theta) .
$$

Moreover, $\mathbf{D}$ can be chosen so that there are only finitely many relational symbols $\varrho \in R$ with $\varrho_{\mathbf{D}} \neq \emptyset$.

2. Let $(\mathbf{C}, \mathbf{D}, E, \theta)$ be a relational configuration over $R$. If there are just finitely many relational symbols $\varrho$ in $R$ with $\varrho_{\mathbf{D}} \neq \emptyset$, then there exists a $\varphi \in \Phi_{|C|}^{(|D|-|C|)}(\exists, \wedge,=, R)$ such that for all R-structures $\mathbf{A}$ and for all $\bar{a} \in A^{|C|}$ we have

$$
\bar{a} \vDash \varphi \text { if and only if } \bar{a} \|(\mathbf{C}, \mathbf{D}, E, \theta) \text {. }
$$

In both cases we say that $\varphi$ corresponds to $(\mathbf{C}, \mathbf{D}, E, \theta)$, and vice versa.

Proof. About 1. Let $\varphi \in \Phi_{m}(\exists, \wedge,=, R)$. Then there exists a finite set $I$ and atomic formulae $\psi_{i}, i \in I$ such that

$$
\varphi \equiv\left(\exists x_{m+1}\right) \ldots\left(\exists x_{m+l}\right) \bigwedge_{i \in I} \psi_{i}
$$

We define $C:=\left\{x_{1}, \ldots, x_{m}\right\}$. Note that free $(\varphi) \subseteq C$. Let, further, $D:=\left\{x_{1}, \ldots, x_{m+l}\right\}$. Moreover, for every $n \in \mathbb{N}$ and $\varrho \in R^{(n)}$, let $\varrho_{\mathbf{D}}:=\left\{\left(x_{j_{1}}, \ldots, x_{j_{n}}\right) \mid \varrho\left(x_{j_{1}}, \ldots, x_{j_{n}}\right) \equiv\right.$ $\psi_{i}$ for some $\left.i \in I\right\}$, let $\theta:=\left\{\left(x_{j_{1}}, x_{j_{2}}\right) \mid\left(x_{j_{1}}=x_{j_{2}}\right) \equiv \psi_{i}\right.$ for some $\left.i \in I\right\}$ and, finally, let $E:\{1, \ldots, m\} \rightarrow C$ be given by $E(i):=x_{i}$. Consider the relational configuration $(\mathbf{C}, \mathbf{D}, E, \theta)$ where $\mathbf{D}=\left(D,\left(\varrho_{\mathbf{D}}\right)_{\varrho \in R}\right)$ and where $\mathbf{C}$ is a substructure of $\mathbf{D}$ induced by $C$. Clearly, there are just finitely many $\varrho \in R$ such that $\varrho_{\mathbf{D}} \neq \emptyset$. We will show now that $(\mathbf{C}, \mathbf{D}, E, \theta)$ corresponds to $\varphi$. Let $\mathbf{A}$ be an $R$-structure and let $\bar{a} \in A^{m}$. 
$(\Rightarrow)$ Suppose that $\bar{a} \vDash \varphi$. Then there exists a valuation $v: X \rightarrow A$ such that $v\left(x_{i}\right)=a_{i}, i=1, \ldots m$, and for every $\psi_{i}$ holds $\mathbf{A} \models_{v} \psi_{i}$.

We define $g: D \rightarrow A$ such that $g\left(x_{i}\right):=v\left(x_{i}\right), i=1, \ldots, m+l$, (i.e. $\left.g:=v \uparrow_{D}\right)$. We will show that such a $g$ is a homomorphism, and that it fulfills (G1) and (G2).

Take any $\varrho \in R$ and $\left(x_{j_{1}}, \ldots, x_{j_{n}}\right) \in \varrho_{\mathbf{D}}$. This means that $\varrho\left(x_{j_{1}}, \ldots, x_{j_{n}}\right) \equiv \psi_{i}$ for some $i \in I$. On the other hand, $\mathbf{A} \models_{v} \psi_{i}$, so $\left(g\left(x_{j_{1}}\right), \ldots, g\left(x_{j_{n}}\right)\right)=\left(v\left(x_{j_{1}}\right), \ldots, v\left(x_{j_{n}}\right)\right) \in$ $\varrho_{\mathrm{A}}$, and, hence, $g$ is a homomorphism.

Note that (G1) follows from the construction of $g$. For (G2) take $\left(x_{j_{1}}, x_{j_{2}}\right) \in \theta$. Then there exists $\psi_{i}$ such that $\psi_{i} \equiv\left(x_{j_{1}}=x_{j_{2}}\right)$. Since $\mathbf{A} \models_{v} \psi_{i}$, it follows that $v\left(x_{j_{1}}\right)=v\left(x_{j_{2}}\right)$, so $\left(x_{j_{1}}, x_{j_{2}}\right) \in$ ker $v$, and, therefore, $\left(x_{j_{1}}, x_{j_{2}}\right) \in \operatorname{ker} g$.

$(\Leftarrow)$ Suppose that $\bar{a} \|(\mathbf{C}, \mathbf{D}, E, \theta)$. Then there exists a homomorphism $g: \mathbf{D} \rightarrow$ A for which hold (G1) and (G2). We define a valuation $v: X \rightarrow A$ in the following way:

$$
v(x)=\left\{\begin{aligned}
g(x), & \text { if } x \in D \\
\text { arbitrary, } & \text { otherwise, }
\end{aligned}\right.
$$

Take any $\psi_{i}, i \in I$. Then either $\psi_{i} \equiv \varrho\left(x_{j_{1}}, \ldots, x_{j_{n}}\right)$, for some $\varrho \in R^{(n)}$ or $\psi_{i} \equiv$ $\left(x_{j_{1}}=x_{j_{2}}\right)$. In the first case we have that $\left(x_{j_{1}}, \ldots, x_{j_{n}}\right) \in \varrho_{\mathbf{D}}$, so $\left(v\left(x_{j_{1}}\right), \ldots, v\left(x_{j_{n}}\right)\right)=$ $\left(g\left(x_{j_{1}}\right), \ldots, g\left(x_{j_{n}}\right)\right) \in \varrho_{\mathbf{A}}$, and, hence, $\mathbf{A} \vDash_{v} \psi_{i}$. In the second, it follows that $\left(x_{j_{1}}, x_{j_{2}}\right) \in \theta$, so $\left(x_{j_{1}}, x_{j_{2}}\right) \in \operatorname{ker} g$, and, therefore, $\left(x_{j_{1}}, x_{j_{2}}\right) \in \operatorname{ker} v$, implying that $\mathbf{A} \models_{v} \psi_{i}$.

About 2. Let $(\mathbf{C}, \mathbf{D}, E, \theta)$ be such that $|C|=m, C=\left\{c_{1}, \ldots c_{m}\right\}, D=\left\{c_{1}, \ldots c_{m+l}\right\}$ and $E(i)=c_{i}, i=1, \ldots, m$. Take an embedding $f: D \rightarrow X$ such that $f\left(c_{i}\right)=x_{i}$, $i=1, \ldots, m+l$ and let

$$
\begin{aligned}
& \varphi \equiv\left(\exists f\left(c_{m+1}\right)\right) \ldots\left(\exists f\left(c_{m+l}\right)\right)\left(\bigwedge_{\substack{\varrho \in R \\
\varrho \mathbf{D} \neq \emptyset}} \bigwedge_{\left(c_{j_{1}}, \ldots, c_{j_{\mathrm{ar}(\varrho)}}\right) \in \varrho_{\mathbf{D}}} \varrho\left(f\left(c_{j_{1}}\right), \ldots, f\left(c_{j_{\operatorname{ar}(\varrho)}}\right)\right)\right. \\
&\left.\wedge \bigwedge_{c_{j_{1}}=c_{j_{2}}}\left(f\left(c_{j_{1}}\right)=f\left(c_{j_{2}}\right)\right)\right) .
\end{aligned}
$$

Let us show now that $\varphi$ corresponds to $(\mathbf{C}, \mathbf{D}, E, \theta)$. Let $\mathbf{A}$ be an $R$-structure and let $\bar{a} \in A^{m}$.

$(\Rightarrow)$ Suppose $\bar{a} \vDash \varphi$. Then there exists a valuation $v: X \rightarrow A$ such that $v\left(x_{i}\right)=a_{i}, i=1, \ldots m$, for every $\varrho \in R$ and every $\left(c_{j_{1}}, \ldots, c_{j_{\operatorname{ar}(\varrho)}}\right) \in \varrho_{\mathbf{D}}$ holds $\mathbf{A} \vDash_{v}$ $\varrho\left(f\left(c_{j_{1}}\right), \ldots, f\left(c_{j_{\operatorname{ar}(\rho)}}\right)\right)$, and for every $\left(c_{j_{1}}, c_{j_{2}}\right) \in \theta$ holds $\mathbf{A} \models_{v}\left(f\left(c_{j_{1}}\right)=f\left(c_{j_{2}}\right)\right)$.

We define $g: D \rightarrow A$ such that $g\left(c_{i}\right):=v\left(f\left(c_{i}\right)\right)$, for $i=1, \ldots, m+l$, and we show that $g$ is a homomorphism that satisfies (G1) and (G2).

Take $\varrho \in R$ and $\left(c_{j_{1}}, \ldots, c_{j_{\operatorname{ar}(\rho)}}\right) \in \varrho_{\mathbf{D}}$. Since $\mathbf{A} \models_{v} \varrho\left(f\left(c_{j_{1}}\right), \ldots, f\left(c_{j_{\mathrm{ar}(\rho)}}\right)\right)$, it follows that $\left(g\left(c_{j_{1}}\right), \ldots, g\left(c_{j_{\operatorname{ar}(\rho)}}\right)\right)=\left(v\left(f\left(c_{j_{1}}\right)\right), \ldots, v\left(f\left(c_{j_{\operatorname{ar}(o)}}\right)\right)\right) \in \varrho_{\mathbf{A}}$, and, hence, $g$ is a homomorphism. For (G1), note that $g\left(c_{i}\right)=v\left(f\left(c_{i}\right)\right)=v\left(x_{i}\right)=a_{i}$. For (G2), take $\left(c_{j_{1}}, c_{j_{2}}\right) \in$ $\theta$. Since $\mathbf{A} \models_{v}\left(f\left(c_{j_{1}}\right)=f\left(c_{j_{2}}\right)\right)$, it follows that $g\left(c_{j_{1}}\right)=v\left(f\left(c_{j_{1}}\right)\right)=v\left(f\left(c_{j_{2}}\right)\right)=g\left(c_{j_{2}}\right)$.

$(\Leftarrow)$ Suppose that $\bar{a} \|(\mathbf{C}, \mathbf{D}, E, \theta)$. Then there exists a homomorphism $g: \mathbf{D} \rightarrow$ A for which hold (G1) and (G2). We define a valuation $v: X \rightarrow A$ in the following 
way:

$$
v(x)= \begin{cases}g\left(f^{-1}(x)\right), & \text { if } x \in f(D) \\ \text { arbitrary, } & \text { otherwise, }\end{cases}
$$

Take any $\left(c_{j_{1}}, \ldots, c_{j_{\operatorname{ar}(\varrho)}}\right) \in \varrho_{\mathbf{D}}$ and consider the subformula $\varrho\left(f\left(c_{j_{1}}\right), \ldots, f\left(c_{j_{\operatorname{ar}(\varrho)}}\right)\right)$ of $\varphi$, that corresponds to it. Then

$$
\begin{aligned}
\left(v\left(f\left(c_{j_{1}}\right)\right), \ldots, v\left(f\left(c_{j_{\operatorname{ar}(\varrho)}}\right)\right)\right) & =\left(g\left(f^{-1}\left(f\left(c_{j_{1}}\right)\right)\right), \ldots, g\left(f^{-1}\left(f\left(c_{j_{\operatorname{ar}(\varrho)}}\right)\right)\right)\right) \\
& =\left(g\left(c_{j_{1}}\right), \ldots, g\left(c_{j_{\operatorname{ar}(\varrho)}}\right)\right) \in \varrho_{\mathbf{A}} .
\end{aligned}
$$

On the other hand, for $\left(c_{j_{1}}, c_{j_{2}}\right) \in \theta$ and the corresponding subformula $\left(f\left(c_{j_{1}}\right)=\right.$ $\left.f\left(c_{j_{2}}\right)\right)$ of $\varphi$ we obtain $v\left(f\left(c_{j_{1}}\right)\right)=g\left(c_{j_{1}}\right)=g\left(c_{j_{2}}\right)=v\left(f\left(c_{j_{2}}\right)\right)$.

Hence, $\bar{a} \vDash \varphi$.

Definition 2. Let $(\mathbf{C}, \mathbf{D}, E, \theta)$ and $(\tilde{\mathbf{C}}, \tilde{\mathbf{D}}, \tilde{E}, \tilde{\theta})$ be such that $|C|=|\tilde{\mathbf{C}}|=m$. We say that $(\mathbf{C}, \mathbf{D}, E, \theta)$ is stronger than $(\tilde{\mathbf{C}}, \tilde{\mathbf{D}}, \tilde{E}, \tilde{\theta})$ in $\mathbf{A}$ if for all $\bar{a} \in A^{m}$ holds

$$
\text { if } \bar{a} \|(\mathbf{C}, \mathbf{D}, E, \theta) \text {, then } \bar{a} \|(\tilde{\mathbf{C}}, \tilde{\mathbf{D}}, \tilde{E}, \tilde{\theta}) \text {. }
$$

\subsection{Galois connections}

Let $\mathbf{A}=\left(A, \leq_{\mathbf{A}}\right)$ and $\mathbf{B}=\left(B, \leq_{\mathbf{B}}\right)$ be two partially ordered sets and let $\alpha: A \rightarrow B$ and $\beta: B \rightarrow A$ be maps. Then the pair $(\alpha, \beta)$ is called a Galois connection between $\mathbf{A}$ and $\mathbf{B}$ if and only for all $a \in A$ and all $b \in B$ we have

$$
a \leq_{\mathrm{A}} \beta(b) \Leftrightarrow b \leq_{\mathrm{B}} \alpha(a) .
$$

We will turn our attention to the case $\mathbf{A}=(\mathcal{P}(G), \subseteq)$ and $\mathbf{B}=(\mathcal{P}(M), \subseteq)$, for sets $G$ and $M$. Any relation between $G$ and $M$ induces a Galois connection between $\mathbf{A}$ and B. Such Galois connections are best studied by using the language of formal concept analysis (cf. [12]).

A formal context is a triple $\mathbb{K}=(G, M, I)$, where $G$ and $M$ are sets, and $I \subseteq G \times M$ is a relation between them. The elements of $G$ we call objects, and the elements of $M$ we call attributes. It is customary to represent small contexts using a cross table. It is a table in which rows correspond to objects, columns correspond to attributes and we write in a field representing the intersection of a row $g$ and a column $m$ a cross if the corresponding object $g$ is in a relation $I$ with the corresponding attribute $m$.

For a set $S \subseteq G$ we define

$$
S^{\prime}:=\{m \mid m \in M \text { and }(\forall g \in S)(g, m) \in I\} .
$$

Analogously, for $T \subseteq M$ we define

$$
T^{\prime}:=\{g \mid g \in G \text { and }(\forall m \in T)(g, m) \in I\} .
$$


A formal concept of the context $\mathbb{K}=(G, M, I)$ is a pair $(S, T)$ such that

$$
S \subseteq G, T \subseteq M, S^{\prime}=T \text { and } T^{\prime}=S .
$$

We say that $S$ is an extent and that $T$ is an intent. The set of extents of $\mathbb{K}$ we denote by $\operatorname{Ext}(\mathbb{K})$ and the set of intents of $\mathbb{K}$ we denote by $\operatorname{Int}(\mathbb{K})$. If we define $\alpha: \mathcal{P}(G) \rightarrow \mathcal{P}(M): S \mapsto S^{\prime}$ and $\beta: \mathcal{P}(M) \rightarrow \mathcal{P}(G): T \mapsto T^{\prime}$, then the pair $(\alpha, \beta)$ is a Galois connection between $(\mathcal{P}(G), \subseteq)$ and $(\mathcal{P}(M), \subseteq)$. From this it follows that $\operatorname{Ext}(\mathbb{K})$ and $\operatorname{Int}(\mathbb{K})$ form closure systems on $G$ and $M$, respectively.

For more information about Galois connections we refer to [9].

\subsection{Clone theory}

We will here introduce only those notions from clone theory that will be needed throughout the thesis. For more information and detailed overview of this field see [44], [40], [53] and [26].

Let $Q$ be a set of relations over a given basic set $A$ and let $R$ be a set of relational symbols such that $|Q|=|R|$. We take a bijection from $R$ to $Q$ that maps $\varrho \in R$ to $\varrho_{\mathbf{A}} \in Q$ and define $\operatorname{ar}(\varrho):=\operatorname{ar}\left(\varrho_{\mathbf{A}}\right)$. Then $R$ with so defined arity function is a relational signature and $\mathbf{A}=\left(A,\left(\varrho_{\mathbf{A}}\right)_{\varrho \in R}\right)$ is a relational structure over a signature $R$. With $Q^{(m)}$ we denote the set of all $m$-ary relations in $Q$. We say that $Q$ is definitionally closed with respect to a set $\Psi \subseteq \bigcup_{m \in \mathbb{N}} \Phi_{m}(\exists, \wedge, \vee,=, R)$ if for every $\varphi \in \Psi$ holds $\varphi^{\mathbf{A}} \in Q$. A set of relations that is definitionally closed with respect to $\bigcup_{m \in \mathbb{N}} \Phi_{m}(\exists, \wedge, \vee,=, R)$ is called a weak Krasner algebra.

Define a context $\left(A^{A}, \bigcup_{n \in \mathbb{N}} \mathcal{P}\left(A^{n}\right), \triangleright\right)$, where $\triangleright$ is the relation of preservation, i.e. for $f \in A^{A}$ and $\varrho \subseteq A^{n}$ we say that $f$ preserves $\varrho$ and write $f \triangleright \varrho$ if

$$
\text { for every }\left(a_{i_{1}}, \ldots, a_{i_{n}}\right) \in \varrho \text { we have }\left(f\left(a_{i_{1}}\right), \ldots, f\left(a_{i_{n}}\right)\right) \in \varrho \text {. }
$$

This context defines a Galois connection (cf. [39]). It is customary to write for set $F$ of functions that $F^{\prime}=\operatorname{Inv} F$ and for set $Q$ of relations $Q^{\prime}=$ End $Q$. It is easy to see that Galois-closed sets of functions are transformation monoids over $A$ and that Galois-closed sets of relations are weak Krasner algebras over $A$. If $A$ is a finite set, then (a part of) the main theorem of clone theory (cf. [40, Hauptsatz 2.1.3.(ii)]) says that every transformation monoid and every weak Krasner algebra over $A$ is Galois-closed. However, if $A$ is infinite, then this is not true in general. Galois-closed transformation monoids and Galois-closed weak Krasner algebras are called locally closed. In locally closed weak Krasner algebras, the $m$-ary relations form a closure system. If $F$ is a set of unary functions on the basic set $A$ and $\varrho \subseteq A^{m}$, then by $\Gamma_{F}(\varrho)$ we denote the smallest $m$-ary relation in Inv $F$ that contains $\varrho$. 
Though this be madness, yet there is method in't.

\section{Chapter 1.}

(William Shakespeare)

\section{Local methods for relational structures}

The main goal of clone theory is to study the structure of the clone lattice. One of the possible approaches to this problem is to partition the clone lattice into monoidal intervals, i.e. intervals over the unary parts of clones. This divides the problem of understanding this lattice into two parts. The first one is to study the structure of monoidal intervals (see [22]), while the second one is to understand the mutual relations between these intervals. One of the main problems that arise from the second part is the following:

Let $\varrho$ and $\sigma$ be the relations over the same basic set $A$.

$$
\text { Decide whether End }\{\varrho\} \subseteq \text { End }\{\sigma\} \text {. }
$$

The same problem arose in the process of finding a completeness criterion for concrete near-rings (see [1] and [27]). In particular, this study involved the problem of describing the structure of the poset of traces of maximal clones. This means that for every two Rosenberg relations one has to decide whether one endomorphism monoid is contained in the other one. A related problem was posed by Pöschel and Radeleczky (see [41]). Namely, they ask to characterize all quasi-order relations that are invariant for a given finite unary algebra. This motivates to study the problem (1) independently.

The initial steps were already done in [30, 33, 34, 37, 38]. However, the methods used were rather ad hoc, depending on the specific relations in question. In this chapter, a unified approach will be developed. The key idea is to use, so called, local method that is based on showing that a necessary and sufficient condition for inclusion between endomorphism monoids of two relations is the existence of certain kind of encoding one of relations into the other one. During the study, it turned out that this inclusion depends mainly on the relation $\varrho$. The efficiency of the decision procedure depends on the choice of the encoding of $\varrho$ in $\sigma$ and the encoding depends only on $\varrho$. The formal description of this method and the way it is used will be described in 1.1.1 and 1.1.2. Although this method can be used in general, here we restrict our attention to the case when $\varrho$ is one of the Rosenberg relations. This choice is motivated by the authors interest in solving the problem of describing the structure of the poset of traces of maximal clones. On the other hand, it also gives a wide collection of nontrivial examples for the use of local methods. Moreover, the first application of local methods was given in [37]. There, local methods were used to prove that for every finite poset $(L, \leq)$ there exists a finite set $A$ and an order-embedding of $(L, \leq)$ into the poset of endomorphism monoids of central relations over $A$. 
In Section 1.2 we give effective criteria for deciding the problem (1), where $\varrho$ is a Rosenberg relation. To each of the six classes of these relations is dedicated a subsection. The subsections are independent from each other, so this part of the chapter can be used in an encyclopedic way. In order to show the possible ways to use local methods, we present two examples in Section 1.3. The first one demonstrates the use of local methods for the constructive enumeration of the ternary relations in the weak Krasner algebra generated by the three element chain. The second example shows how local methods can be used in order to answer one of the open problems from [30]. In particular, we show that there exists an $h$-regular relation $\varrho$ and a central relation $\sigma$ such that End $\{\varrho\} \subseteq \operatorname{End}\{\sigma\}$. The results from this chapter are going to appear in [32].

Different than in the rest of this thesis, in this chapter we will restrict our attention to relational structures over finite sets. Moreover, the relational signature will usually consist of one relational symbol. Slightly abusing the notation we will identify a relation $\varrho$ over $A$ with the relational structure $(A, \varrho)$.

\subsection{Locality Principle}

The Locality Principle is a way of defining closure systems. In general, we are given a space of points $\mathbf{A}$ with some structure, and an ordered set $\mathbf{T}=(\mathbb{T}, \sqsubseteq)$ of possible observations (called types) that can be made by an observer in the space from a given point. Here an observer is a function that maps each point of the space to the observation that the observer makes standing at the given point. Such kind of function we will denote by type ${ }_{\mathrm{A}}$ and we will usually call it a type-function.

The Locality Principle defines a set of implications on the space A as follows:

For points $\bar{a}$ and $\bar{b}$ the implication $\bar{a} \rightarrow \bar{b}$ holds if type $\mathbf{A}_{\mathbf{A}}(\bar{a}) \sqsubseteq \operatorname{type}_{\mathbf{A}}(\bar{b})$.

This defines a closure system on the space $\mathbf{A}$ and we say that this closure system is defined by the Locality Principle. If two different observers define the same closure system, then we call them equivalent.

In the sequel we will describe specific given closure systems using the Locality Principle. This means that we will find a suitable observer and a poset of observations such that the closure system defined by the Locality Principle is equal to the given one. Of course, this is possible only if the given closure system is definable by the set of implications with precisely one premise, that is, it is algebraic and it induces a distributive lattice.

Note that on every space $\mathbf{A}$ with a given algebraic distributive closure system there exists a trivial observer that maps every point to its closure, where the order on the observations is the reversed inclusion order. However the definition of this observer makes direct use of the given closure system, while the goal of the Locality Principle is to describe a given closure system using as little as possible information about its internal structure. Often it is possible to give a much simpler observer than the above described trivial one. 
1.1.1. Local methods, types and type-ordering. As it was already stated, this study was motivated by the problem of deciding for two given relations $\varrho$ and $\sigma$ over the same finite set $A$, whether End $\{\varrho\} \subseteq \operatorname{End}\{\sigma\}$ without computing the endomorphisms. The classical methods of study of the endomorphism monoids are not so convenient for answering this question, and therefore there is a need to introduce a new approach to this problem. Of course, this requires the use of new research tools and the research tools that are going to be used here are known under the name of local methods. There is no formal definition of a local method, but there is a general idea what is understood under this notion. This is nothing else but the way of understanding the huge structure that is hard to comprehend, by looking it on small pieces that are beautiful enough to be understood, but still sufficient to reconstruct the initial structure. Such a "definition" leaves us a lot of freedom to adjust this notion for our own needs, and we continue with a proposal of a local method that might help us to answer the initial question.

Our proposal is based on the idea that stands behind the Locality Principle. We are given a relation $\varrho$ on the finite basic set $A$. Our goal is to describe the closure system (Inv End $\{\varrho\})^{(m)}$, for $m \in \mathbb{N}$, using the Locality Principle. It is clear that the space is consisting of the $m$-tuples over $A$. We are now looking for the appropriate observer and the poset of observations. For a given poset of observations $(\mathbb{T}, \sqsubseteq)$, a function type $e_{\rho}: A^{m} \rightarrow \mathbb{T}$ is called a type-function for $\varrho$ and $m$ if the closure system that is defined by type $e_{\varrho}$ on $A^{m}$ using the Locality Principle is equal to (Inv End $\{\varrho\})^{(m)}$. Clearly, this is the case precisely when for every $\sigma \subseteq A^{m}$ holds $\sigma \in(\operatorname{Inv} \operatorname{End}\{\varrho\})^{(m)}($ i.e. End $\{\varrho\} \subseteq \operatorname{End}\{\sigma\})$ if and only if

$$
\text { for every } \bar{a}, \bar{b} \in A^{m} \text { if } \bar{a} \in \sigma \text { and type } \varrho_{\varrho}(\bar{a}) \sqsubseteq \operatorname{type}_{\varrho}(\bar{b}) \text { then } \bar{b} \in \sigma \text {. }
$$

1.1.2. Type-functions and the structure of weak Krasner algebras. Local methods which use type functions are giving us a test that applied to arbitrary $m$-ary relation $\sigma$ decides whether it belongs to the weak Krasner algebra generated by a given relation $\varrho$. This kind of test was already used in [37] in the study on the poset of endomorphism monoids of central relations.

Another application is based on the following observations:

Let $\varrho$ be a relation and let type $e_{\varrho}$ be a type-function for $\varrho$ and $m$. For every $\bar{a}=\left(a_{1}, \ldots, a_{m}\right) \in A^{m}$, we associate to the type ${ }_{\varrho}\left(a_{1}, \ldots, a_{m}\right)$ a relation

$$
\sigma_{\text {type }_{\varrho}(\bar{a})}=\left\{\left(b_{1}, \ldots, b_{m}\right) \mid b_{1}, \ldots, b_{m} \in A \wedge \operatorname{type}_{\varrho}\left(a_{1}, \ldots, a_{m}\right) \sqsubseteq \operatorname{type}_{\varrho}\left(b_{1}, \ldots, b_{m}\right)\right\} .
$$

Observe that in this way we associate to distinct types distinct relations. Namely, if we suppose that for type $\operatorname{la}_{\varrho}(\bar{a}) \neq \operatorname{type}_{\varrho}(\bar{b})$, holds that $\sigma_{\text {type }_{\varrho}(\bar{a})}=\sigma_{\text {type }_{\varrho}(\bar{b})}$, then for every $\bar{c} \in A^{m}$ we obtain

$$
\operatorname{type}_{\varrho}(\bar{a}) \sqsubseteq \operatorname{type}_{\varrho}(\bar{c}) \Leftrightarrow \operatorname{type}_{\varrho}(\bar{b}) \sqsubseteq \operatorname{type}_{\varrho}(\bar{c}) .
$$

In particular, it must hold

$$
\operatorname{type}_{\varrho}(\bar{a}) \sqsubseteq \operatorname{type}_{\varrho}(\bar{b}) \text { and } \operatorname{type}_{\varrho}(\bar{b}) \sqsubseteq \operatorname{type}_{\varrho}(\bar{a}),
$$


so type $_{\varrho}(\bar{a})=$ type $_{\varrho}(\bar{b})$ and we arrive at a contradiction.

Lemma. For every relation $\theta \in(\operatorname{Inv} \operatorname{End}\{\varrho\})^{(m)}$ holds

$$
\theta=\bigcup_{\bar{b} \in \theta} \sigma_{\text {type }_{e}(\bar{b})} \text {. }
$$

Proof. Note that for every $\bar{b} \in \theta$ holds $\bar{b} \in \sigma_{\text {type }_{e}(\bar{b})}$. On the other hand, from the definition of a type function it follows that for $\bar{b} \in \theta, \sigma_{\text {type }_{e}(\bar{b})} \subseteq \theta$, so the equality holds.

Proposition 1. For every $\bar{a}, \bar{b} \in A^{m}$

$$
\text { type }_{\varrho}(\bar{a}) \sqsubseteq \text { type }_{\varrho}(\bar{b}) \text { if and only if } \sigma_{\text {type }_{\varrho}(\bar{a})} \supseteq \sigma_{\text {type }_{\varrho}(\bar{b})} \text {. }
$$

Proof. Let type $e_{\varrho}(\bar{a}) \sqsubseteq$ type $_{\varrho}(\bar{b})$. Then for every $\bar{c} \in \sigma_{\text {type }_{\varrho}(\bar{b})}$ we have

$$
\operatorname{type}_{\varrho}(\bar{a}) \sqsubseteq \operatorname{type}_{\varrho}(\bar{b}) \sqsubseteq \operatorname{type}_{\varrho}(\bar{c}),
$$

so $\bar{c} \in \sigma_{\text {type }_{e}(\bar{a})}$.

For the other direction, let $\sigma_{\text {type }_{e}(\bar{a})} \supseteq \sigma_{\text {type }_{e}(\bar{b})}$. Then for every $\bar{c} \in A^{m}$ holds

$$
\text { if type } e_{\varrho}(\bar{b}) \sqsubseteq \text { type }_{\varrho}(\bar{c}) \text {, then type } e_{\varrho}(\bar{a}) \sqsubseteq \text { type }_{\varrho}(\bar{c}) .
$$

Specially, for $\bar{c}=\bar{b}$, we obtain type $e_{\varrho}(\bar{a}) \sqsubseteq \operatorname{type}_{\varrho}(\bar{b})$.

Proposition 2. Every join-irreducible element in the lattice of $(\operatorname{Inv} \operatorname{End}\{\varrho\})^{(m)}$ is of the form $\sigma_{\text {type }_{e}(\bar{a})}$, for some $\bar{a} \in A^{m}$. Moreover, for every $\bar{a} \in A^{m}, \sigma_{\text {type }_{e}(\bar{a})}$ is a join-irreducible element in this lattice.

Proof. Let $\theta$ be an arbitrary join-irreducible element in the given lattice. By the previous Lemma

$$
\theta=\bigcup_{\bar{b} \in \theta} \sigma_{\text {type }_{e}(\bar{b})},
$$

so it follows that $\theta=\sigma_{\text {type }_{e}(\bar{b})}$, for some $\bar{b} \in \theta$, which proves the first claim.

For the proof of the second claim, define

$$
\tilde{\theta}:=\left\{\bar{b} \in A^{m} \mid \operatorname{type}_{\varrho}(\bar{a}) \sqsubset \operatorname{type}_{\varrho}(\bar{b})\right\} .
$$

Note that $\bar{a} \notin \tilde{\theta}$, so $\tilde{\theta} \subset \sigma_{\text {type }_{e}(\bar{a})}$. We will show that for every relation $\sigma \in$ $(\operatorname{Inv} \operatorname{End}\{\varrho\})^{(m)}$ such that $\sigma \subset \sigma_{\text {type }_{\ell}(\bar{a})}$ holds $\sigma \subseteq \tilde{\theta}$. Observe that

$$
\sigma=\bigcup_{\bar{b} \in \sigma} \sigma_{\text {type }_{\rho}(\bar{b})}
$$


so for every $\bar{b} \in \sigma, \sigma_{\text {type }_{\varrho}(\bar{b})} \subset \sigma_{\text {type }_{\varrho}(\bar{a})}$, implying that type $\varrho_{\varrho}(\bar{a}) \sqsubset$ type $_{\varrho}(\bar{b})$, so $\sigma \subseteq \tilde{\theta}$.

Since $A$ is finite, there are finitely many such relations $\sigma$ that are strictly below $\sigma_{\text {type }_{\rho}(\bar{a})}$. All of them are below $\tilde{\theta}$, so the same holds for their union, which is an element of $(\operatorname{Inv} \text { End }\{\varrho\})^{(m)}$. It is then strictly below $\sigma_{\text {type }_{\varrho}(\bar{a})}$, which shows that $\sigma_{\text {type }_{\varrho}(\bar{a})}$ is indeed a join-irreducible element.

It is clear now that types of $m$-ary tuples correspond one-to-one to joinirreducible elements in the lattice of $(\operatorname{Inv} \text { End }\{\varrho\})^{(m)}$.

Corollary. The poset of join-irreducible elements in $(\operatorname{Inv} \text { End }\{\varrho\})^{(m)}$ is dually isomorphic to the poset of types of m-ary tuples.

(Inv End $\{\varrho\})^{(m)}$ is a finite distributive lattice. Therefore, by Birkhoff's representation theorem, it is isomorphic to the lattice of down-sets of the poset of join-irreducible elements. Hence, it is isomorphic to the lattice of order-filters of types of $m$-ary tuples. Counting order-filters in the poset of types is equivalent to counting the antichains in it, so the number of elements in (Inv End $\{\varrho\})^{(m)}$ is equal to the number of antichains in the poset of types. This will be demonstrated in Section 1.3 .

\subsection{Local methods for Rosenberg relations}

We continue by defining type-functions type ${ }_{\rho^{\prime}}$, where $\varrho$ ranges through the set of Rosenberg relations and the basic set $A$ is finite. We start with classes of equivalence and central relations, as well as lattice-orders, because they allow a simple generic definition of types. Then we continue with permutational and affine relations, and end with $h$-regular relations. The general case of boundedpartial orders is not treated here, because until now I could not find a satisfactory generic definition of types for them.

1.2.1. Equivalence relations. Let $\varrho$ be an equivalence relation over a set $A$, let $m \in \mathbb{N}$ and let $\bar{a}=\left(a_{1}, \ldots, a_{m}\right) \in A^{m}$. Then we define

$$
\begin{aligned}
\tau_{1}(\bar{a}) & =\left\{(i, j) \mid i, j \in\{1, \ldots, m\} \wedge\left(a_{i}, a_{j}\right) \in \varrho\right\}, \\
\tau_{2}(\bar{a}) & =\left\{(i, j) \mid i, j \in\{1, \ldots, m\} \wedge a_{i}=a_{j}\right\}, \\
\operatorname{type}_{\varrho}(\bar{a}) & =\left(\tau_{1}(\bar{a}), \tau_{2}(\bar{a})\right) .
\end{aligned}
$$

For $\bar{a}, \bar{b} \in A^{m}$ we write type ${ }_{\varrho}(\bar{a}) \sqsubseteq \operatorname{type}_{\varrho}(\bar{b})$ if $\tau_{1}(\bar{a}) \subseteq \tau_{1}(\bar{b})$ and $\tau_{2}(\bar{a}) \subseteq \tau_{2}(\bar{b})$.

Proposition. The function type $e_{\varrho}$ is a type-function for the relation $\varrho$ and the integer $m$.

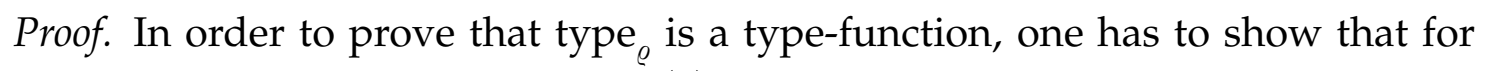
every $\sigma \subseteq A^{m}$ holds $\sigma \in(\operatorname{Inv} \operatorname{End}\{\varrho\})^{(m)}$ if and only if 1.1.1(1) holds.

$(\Leftarrow)$ Let $1.1 .1(1)$ hold. Take $f \in \operatorname{End}\{\varrho\}$ and $\bar{a} \in \sigma$. We define $\bar{b}=f(\bar{a})$, i.e. $b_{i}=f\left(a_{i}\right), i \in\{1, \ldots, m\}$. Our goal is to show that $\bar{b} \in \sigma$, so it suffices to show that type $_{\varrho}(\bar{a}) \sqsubseteq$ type $_{\varrho}(\bar{b})$ holds. 
- Let $\left(a_{i}, a_{j}\right) \in \varrho$. Then $(i, j) \in \tau_{1}(\bar{a})$. Since $f \in \operatorname{End}\{\varrho\}$, it follows that $\left(b_{i}, b_{j}\right)=$ $\left(f\left(a_{i}\right), f\left(a_{j}\right)\right) \in \varrho$, so $(i, j) \in \tau_{1}(\bar{b})$.

- Let $a_{i}=a_{j}$, i.e. $(i, j) \in \tau_{2}(\bar{a})$. Then $f\left(a_{i}\right)=f\left(a_{j}\right)$, so $b_{i}=b_{j}$, and therefore, $(i, j) \in \tau_{2}(\vec{b})$.

Hence, $\bar{b} \in \sigma$, and $f \in \operatorname{End}\{\sigma\}$.

$(\Rightarrow)$ Let End $\{\varrho\} \subseteq$ End $\{\sigma\}$. Take $\bar{a} \in \sigma$ and $\bar{b} \in A^{m}$ such that type $_{\varrho}(\bar{a}) \sqsubseteq$ type $_{\varrho}(\bar{b})$. We will construct an endomorphism of $\sigma$ that takes $\bar{a}$ to $\bar{b}$. For this purpose we first define a function

$$
\mu:\{1, \ldots, m\} \rightarrow\{1, \ldots, m\}: j \mapsto \min \left\{k \mid\left[a_{j}\right]_{\varrho}=\left[a_{k}\right]_{\varrho}\right\}
$$

and then we can define the mapping $f$ in the following way:

$$
f(x)= \begin{cases}b_{i}, & \text { if } x=a_{i}, i \in\{1, \ldots, m\} \\ b_{\mu(j),} & \text { if } x \in\left[a_{j}\right]_{\varrho} \text { and } x \notin\left\{a_{1}, \ldots, a_{m}\right\} \\ b_{1}, & \text { otherwise. }\end{cases}
$$

Clearly, $f$ is well-defined and takes $\bar{a}$ to $\bar{b}$. It is left to show that $f \in \operatorname{End}\{\varrho\}$ :

Let $(x, y) \in \varrho$.

- If $x=a_{i}, y=a_{j}$, then $(i, j) \in \tau_{1}(\bar{a})$, so $(i, j) \in \tau_{1}(\bar{b})$ and, hence, $\left(f\left(a_{i}\right), f\left(a_{j}\right)\right)=$ $\left(b_{i}, b_{j}\right) \in \varrho$.

- If $x=a_{i}, y \in\left[a_{j}\right]_{\varrho}, y \notin\left\{a_{1}, \ldots, a_{m}\right\}$, then $(i, j) \in \tau_{1}(\bar{a})$, so $(i, j) \in \tau_{1}(\bar{b})$ and $\left(b_{i}, b_{j}\right) \in \varrho$. On the other hand, $\left(a_{j}, a_{\mu(j)}\right) \in \varrho$, so $\left(b_{j}, b_{\mu(j)}\right) \in \varrho$, and it follows that $(f(x), f(y))=\left(b_{i}, b_{\mu(j)}\right) \in \varrho$.

- If $x \in\left[a_{j}\right]_{\varrho}, y=a_{i}, x \notin\left\{a_{1}, \ldots, a_{m}\right\}$, then one proves it analogously to the previous case.

- If $x \in\left[a_{i}\right]_{\varrho}, y \in\left[a_{j}\right]_{\varrho}$ and $x, y \notin\left\{a_{1}, \ldots, a_{m}\right\}$, then $\left(a_{i}, a_{j}\right) \in \varrho$ and $(f(x), f(y))=$ $\left(b_{\mu(i)}, b_{\mu(j)}\right)$. By the same reasoning as above, we obtain $\left(b_{\mu(i)}, b_{i}\right) \in \varrho$ and $\left(b_{j}, b_{\mu(j)}\right) \in \varrho$. Since we have that $\left(b_{i}, b_{j}\right) \in \varrho$, it follows that $\left(b_{\mu(i)}, b_{\mu(j)}\right) \in \varrho$.

- Note that the case $x \in\left[a_{i}\right]_{\varrho}, y \notin \bigcup_{i=1}^{m}\left[a_{i}\right]_{\varrho}$ cannot appear, so it remains the case $x, y \notin \bigcup_{i=1}^{m}\left[a_{i}\right]_{\varrho}$. But, then $(f(x), f(y))=\left(b_{1}, b_{1}\right) \in \varrho$.

To conclude, $f \in \operatorname{End}\{\varrho\} \subseteq \operatorname{End}\{\sigma\}$, so $\bar{b} \in \sigma$.

1.2.2. Central relations. A relation $\varrho \subseteq A^{n}$ is said to be totally symmetric if $\left(x_{1}, \ldots, x_{n}\right) \in \varrho$ implies $\left(x_{1}, \ldots, x_{n}\right)^{\pi} \in \varrho$ for all permutations $\pi$, and it is said to be totally reflexive if it contains all the reflexive elements from $A^{n}$. For a totally symmetric relation $\varrho$, an element $c \in A$ is called central if $\left(c, x_{2}, \ldots, x_{n}\right) \in \varrho$ for all $x_{2}, \ldots, x_{n} \in A$. The set $C_{\varrho}$ of all central elements of $\varrho$ is called center. Finally, 
$\varrho \neq A^{n}$ is called central relation if it is totally reflexive, totally symmetric and has at least one central element.

Note that the unary central relations correspond just to the proper nonempty subsets of $A$.

Let $\varrho$ be an $n$-ary central relation over a set $A$, let $m$ be a natural number and let $\bar{a}=\left(a_{1}, \ldots, a_{m}\right) \in A^{m}$. We define

$$
\begin{aligned}
\tau_{1}(\bar{a}) & =\left\{\left(i_{1}, \ldots, i_{n}\right) \mid i_{1}, \ldots, i_{n} \in\{1, \ldots, m\} \wedge\left(a_{i_{1}}, \ldots, a_{i_{n}}\right) \in \varrho\right\}, \\
\tau_{2}(\bar{a}) & =\left\{(i, j) \mid i, j \in\{1, \ldots, m\} \wedge a_{i}=a_{j}\right\}, \\
\operatorname{type}_{\varrho}(\bar{a}) & =\left(\tau_{1}(\bar{a}), \tau_{2}(\bar{a})\right) .
\end{aligned}
$$

For $\bar{a}, \bar{b} \in A^{m}$ we write type ${ }_{\varrho}(\bar{a}) \sqsubseteq \operatorname{type}_{\varrho}(\bar{b})$ if $\tau_{1}(\bar{a}) \subseteq \tau_{1}(\bar{b})$ and $\tau_{2}(\bar{a}) \subseteq \tau_{2}(\bar{b})$.

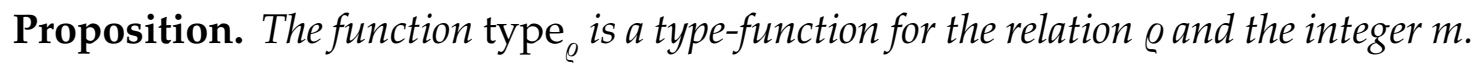

Proof. In order to prove that type ${ }_{\rho}$ is a type-function, one has to show that for every $\sigma \subseteq A^{m}$ holds $\sigma \in(\operatorname{Inv} \operatorname{End}\{\varrho\})^{(m)}$ if and only if 1.1.1(1) holds.

$(\Leftarrow)$ Let 1.1.1(1) hold. Take $f \in \operatorname{End}\{\varrho\}$ and $\bar{a} \in \sigma$. We define $\bar{b}=f(\bar{a})$, i.e. $b_{i}=f\left(a_{i}\right), i \in\{1, \ldots, m\}$. We will show that $\bar{b} \in \sigma$.

Since 1.1.1(1) holds, it suffices to show that type $e_{\varrho}(\bar{a}) \sqsubseteq$ type $_{\varrho}(\bar{b})$ holds, i.e. $\tau_{1}(\bar{a}) \subseteq \tau_{1}(\bar{b})$ and $\tau_{2}(\bar{a}) \subseteq \tau_{2}(\bar{b})$.

- Let $\left(a_{i_{1}}, \ldots, a_{i_{n}}\right) \in \varrho$, i.e $\left(i_{1}, \ldots, i_{n}\right) \in \tau_{1}(\bar{a})$. Since $f \in$ End $\{\varrho\}$, it follows that $\left(b_{i_{1}}, \ldots, b_{i_{n}}\right)=\left(f\left(a_{i_{1}}\right), \ldots, f\left(a_{i_{n}}\right)\right) \in \varrho$, so $\left(i_{1}, \ldots, i_{n}\right) \in \tau_{1}(\bar{b})$.

- Let $a_{i}=a_{j}$, i.e. $(i, j) \in \tau_{2}(\bar{a})$. Then $f\left(a_{i}\right)=f\left(a_{j}\right)$, so $b_{i}=b_{j}$, and therefore, $(i, j) \in \tau_{2}(\vec{b})$.

Hence, $\bar{b} \in \varrho$, and $f \in \operatorname{End}\{\sigma\}$.

$(\Rightarrow)$ Let End $\{\varrho\} \subseteq$ End $\{\sigma\}$. Take $\bar{a} \in \sigma$ and $\bar{b} \in A^{m}$ such that type $_{\varrho}(\bar{a}) \sqsubseteq \operatorname{type}_{\varrho}(\bar{b})$. We will construct an endomorphism of $\sigma$ that takes $\bar{a}$ to $\bar{b}$. Let $c \in C_{\varrho}$. We define a mapping $f$ in the following way:

$$
f(x)= \begin{cases}b_{i}, & \text { if } x=a_{i}, i \in\{1, \ldots, m\} \\ c, & \text { otherwise }\end{cases}
$$

Clearly, $f$ is well-defined and takes $\bar{a}$ to $\bar{b}$. It is left to show that $f \in \operatorname{End}\{\varrho\}$. It will be enough to show that $f$ maps irreflexive elements of $\varrho$ into $\varrho$. So, let $\bar{x}=\left(x_{1}, \ldots, x_{n}\right) \in \varrho^{i r r}$.

- If $\left(x_{1}, \ldots, x_{n}\right)=\left(a_{i_{1}}, \ldots, a_{i_{n}}\right)$, for some $i_{1}, \ldots, i_{n}$, then $\left(i_{1}, \ldots, i_{n}\right) \in \tau_{1}(\bar{a})$. Hence, $\left(i_{1}, \ldots, i_{n}\right) \in \tau_{1}(\bar{b})$, so $f(\bar{x})=\left(b_{i_{1}}, \ldots, b_{i_{n}}\right) \in \varrho$.

- If, however, there is an $i \in\{1, \ldots, n\}$, such that $x_{i} \notin\left\{a_{1}, \ldots, a_{m}\right\}$, then $f\left(x_{i}\right)=c$. Hence, $f(\bar{x})$ contains a central element of $\varrho$ as an entry, so $f(\bar{x}) \in \varrho$.

To conclude, $f \in \operatorname{End}\{\varrho\} \subseteq \operatorname{End}\{\sigma\}$, so $\bar{b} \in \sigma$. 
1.2.3. Lattice-orders. Let $\varrho$ be a lattice-order over a set $A$, let $m \in \mathbb{N}$ and let $\bar{a}=\left(a_{1}, \ldots, a_{m}\right) \in A^{m}$. Instead of writing $(x, y) \in \varrho$, we will use the more intuitive notation $x \leq_{\varrho} y$. Also, we denote by $0_{\varrho}$ the smallest element of this ordered set, and by $x \downarrow=\left\{z \in A \mid z \leq_{\varrho} x\right\}$ the down-set of $x$. We define

$$
\begin{aligned}
\tau_{1}(\bar{a}) & =\left\{(i, j) \mid i, j \in\{1, \ldots, m\} \wedge a_{i} \leq_{\varrho} a_{j}\right\}, \\
\tau_{2}(\bar{a}) & =\left\{(i, j) \mid i, j \in\{1, \ldots, m\} \wedge a_{i}=a_{j}\right\}, \\
\operatorname{type}_{\varrho}(\bar{a}) & =\left(\tau_{1}(\bar{a}), \tau_{2}(\bar{a})\right) .
\end{aligned}
$$

For $\bar{a}, \bar{b} \in A^{m}$ we write type ${ }_{\varrho}(\bar{a}) \sqsubseteq \operatorname{type}_{\varrho}(\bar{b})$ if $\tau_{1}(\bar{a}) \subseteq \tau_{1}(\bar{b})$ and $\tau_{2}(\bar{a}) \subseteq \tau_{2}(\bar{b})$.

Proposition. The function type $e_{\varrho}$ is a type-function for the relation $\varrho$ and the integer $m$.

Proof. In order to prove that type ${ }_{\varrho}$ is a type-function, one has to show that for every $\sigma \subseteq A^{m}$ holds $\sigma \in(\operatorname{Inv} \operatorname{End}\{\varrho\})^{(m)}$ if and only if 1.1.1(1) holds.

$(\Leftarrow)$ Let 1.1.1(1) hold. Take $f \in \operatorname{End}\{\varrho\}$, and $\bar{a} \in \sigma$. We define $\bar{b}=f(\bar{a})$, i.e. $b_{i}=f\left(a_{i}\right), i \in\{1, \ldots, m\}$. We will show that $\bar{b} \in \sigma$.

Since 1.1.1(1) holds, it suffices to show that type $e_{\varrho}(\bar{a}) \sqsubseteq$ type $_{\varrho}(\bar{b})$ holds.

- Let $a_{i} \leq{ }_{\varrho} a_{j}$. Then $(i, j) \in \tau_{1}(\bar{a})$. Since $f \in \operatorname{End}\{\varrho\}$, it follows that $\left(b_{i}, b_{j}\right)=$ $\left(f\left(a_{i}\right), f\left(a_{j}\right)\right) \in \varrho$, so $(i, j) \in \tau_{1}(\bar{b})$.

- Let $a_{i}=a_{j}$, i.e. $(i, j) \in \tau_{2}(\bar{a})$. Then $f\left(a_{i}\right)=f\left(a_{j}\right)$, so $b_{i}=b_{j}$, and therefore, $(i, j) \in \tau_{2}(\vec{b})$.

Hence, $\bar{b} \in \varrho$, and $f \in \operatorname{End}\{\sigma\}$.

$(\Rightarrow)$ Let End $\{\varrho\} \subseteq$ End $\{\sigma\}$. Let $\bar{a} \in \sigma$ and $\bar{b} \in A^{m}$ be such that type ${ }_{\rho}(\bar{a}) \sqsubseteq \operatorname{type}_{\varrho}(\bar{b})$. We will construct an endomorphism of $\varrho$ that takes $\bar{a}$ to $\bar{b}$. Define the mapping $f$ in the following way:

$$
f(x)= \begin{cases}b_{i}, & \text { if } x=a_{i}, i \in\{1, \ldots, m\} \\ \sup f\left(x \downarrow \cap\left\{a_{1}, \ldots, a_{m}\right\}\right), & \text { otherwise. }\end{cases}
$$

(Note that $\sup \emptyset=0_{\varrho}$ ).

Clearly, this mapping is well-defined and by definition takes $\bar{a}$ to $\bar{b}$, so it is left to show that it is an endomorphism of the lattice-order:

Let $(x, y) \in \varrho$, i.e. $x \leq_{\varrho} y$.

- If $x=a_{i}, y=a_{j}$, then $(i, j) \in \tau_{1}(\bar{a})$, so $(i, j) \in \tau_{1}(\bar{b})$ and, hence, $\left(f\left(a_{i}\right), f\left(a_{j}\right)\right)=$ $\left(b_{i}, b_{j}\right) \in \varrho$.

- If $x=a_{i}$, and $y \in A \backslash\left\{a_{1}, \ldots, a_{m}\right\}$, then we have that $f(x)=b_{i}$, and $f(y)=\sup f\left(y \downarrow \cap\left\{a_{1}, \ldots, a_{m}\right\}\right)$. From $a_{i} \leq_{\varrho} y$, it follows that $a_{i} \in y \downarrow$, so $a_{i} \in y \downarrow \cap\left\{a_{1}, \ldots, a_{m}\right\}$. Applying $f$ on both sides, we obtain that $f\left(a_{i}\right) \in f\left(y \downarrow \cap\left\{a_{1}, \ldots, a_{m}\right\}\right)$, so $f\left(a_{i}\right) \leq_{\varrho} \sup f\left(y \downarrow \cap\left\{a_{1}, \ldots, a_{m}\right\}\right)$, i.e. $f(x) \leq_{\varrho} f(y)$. 
- If $x \in A \backslash\left\{a_{1}, \ldots, a_{m}\right\}, y=a_{i}$, then from $x \leq_{\varrho} a_{i}$, it follows that for every $z \in x \downarrow$ holds $z \leq_{\varrho} a_{i}$, so for every $a_{j} \in x \downarrow \cap\left\{a_{1}, \ldots, a_{m}\right\}$ holds $a_{j} \leq_{\varrho} a_{i}$, i.e. $(j, i) \in\left\{(i, j) \mid i, j \in\{1, \ldots, m\} \wedge a_{i} \leq_{\varrho} a_{j}\right\}$. Since type ${ }_{\varrho}(\bar{a}) \sqsubseteq$ type $_{\varrho}(\bar{b})$, it follows that $b_{j} \leq_{\varrho} b_{i}$, so $f\left(a_{j}\right) \leq_{\varrho} f\left(a_{i}\right)$. So, $\sup f\left(x \downarrow \cap\left\{a_{1}, \ldots, a_{m}\right\}\right) \leq_{\varrho} f\left(a_{i}\right)$, i.e. $f(x) \leq_{\varrho} f(y)$.

- If $x, y \in A \backslash\left\{a_{1}, \ldots, a_{m}\right\}$, then $f(x)=\sup f\left(x \downarrow \cap\left\{a_{1}, \ldots, a_{m}\right\}\right)$. From $x \leq_{\varrho} y$, it follows that $x \in y \downarrow$, i.e. $x \downarrow \subseteq y \downarrow$. So, $x \downarrow \cap\left\{a_{1}, \ldots, a_{m}\right\} \subseteq y \downarrow \cap\left\{a_{1}, \ldots, a_{m}\right\}$, and hence, $f\left(x \downarrow \cap\left\{a_{1}, \ldots, a_{m}\right\}\right) \subseteq f\left(y \downarrow \cap\left\{a_{1}, \ldots, a_{m}\right\}\right)$. Therefore, sup $f(x \downarrow$ $\left.\cap\left\{a_{1}, \ldots, a_{m}\right\}\right) \leq_{\varrho} \sup f\left(y \downarrow \cap\left\{a_{1}, \ldots, a_{m}\right\}\right)$, and, finally, $f(x) \leq_{\varrho} f(y)$.

To conclude, $f \in \operatorname{End}\{\varrho\} \subseteq \operatorname{End}\{\sigma\}$, and hence, $\bar{b} \in \sigma$.

As it was already mentioned above, there is still no satisfactory generic definition of types for bounded partial orders. The following example shows why the types that solved the problem for the lattice orders are not functioning for arbitrary bounded partial orders.

Example. On the core of the characterization lies the fact that if we have $\bar{a}, \bar{b} \in A^{m}$ such that type ${ }_{\rho}(\bar{a}) \sqsubseteq$ type $_{\rho}(\bar{b})$ then there exists an endomorphism of the given bounded partial order that maps $\bar{a}$ to $\bar{b}$. Consider the bounded partial order $\varrho$ given in (1) and the smallest relation in Inv End $\{\varrho\}$ that contains the tuple $\left(a_{1}, a_{2}, a_{3}, a_{4}\right)$. This relation is equal to

$$
\sigma:=\left\{\left(f\left(a_{1}\right), f\left(a_{2}\right), f\left(a_{3}\right), f\left(a_{4}\right)\right) \mid f \in \operatorname{End}\{\varrho\}\right\} .
$$

We show that $\left(a_{6}, a_{7}, a_{8}, a_{9}\right) \notin \sigma$ even though

$$
\operatorname{type}_{\varrho}\left(\left(a_{1}, a_{2}, a_{3}, a_{4}\right)\right) \sqsubseteq \operatorname{type}_{\varrho}\left(\left(a_{6}, a_{7}, a_{8}, a_{9}\right)\right) .
$$

Suppose that $\left(a_{6}, a_{7}, a_{8}, a_{9}\right) \in \sigma$ and take $f \in$ End $\{\varrho\}$ such that $f\left(a_{1}\right)=a_{6}, f\left(a_{2}\right)=a_{7}, f\left(a_{3}\right)=a_{8}$ and $f\left(a_{4}\right)=a_{9}$. The image of $a_{5}$ should be then below $a_{6}$ and $a_{7}$, but above $a_{8}$ and $a_{9}$. Since such an element does not exist, it follows that $\left(a_{6}, a_{7}, a_{8}, a_{9}\right) \notin \sigma$. Therefore, $f$ is not an endomorphism of $\sigma$.

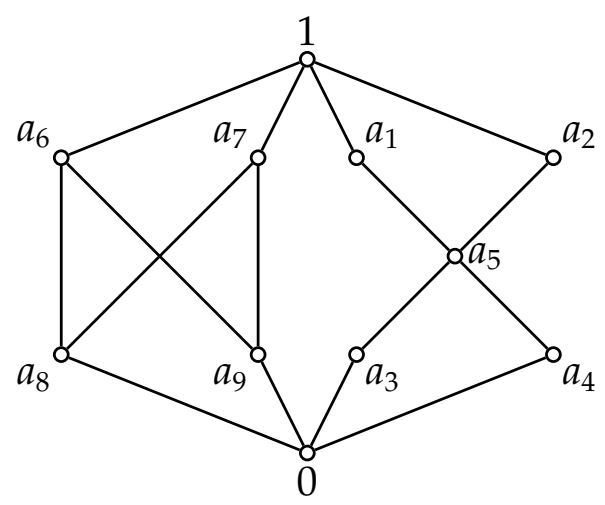

1.2.4. Permutational relations. A relation $\varrho$ is called permutational relation if it is of the form $\left\{\left(x, x^{\pi}\right) \mid x \in A\right\}$, where $\pi$ is a fix-point free permutation of $A$ that has prime order $p$. 
Let $\varrho$ be a permutational relation over a set $A$, let $m$ be a natural number and let $\bar{a}=\left(a_{1}, \ldots, a_{m}\right) \in A^{m}$. Let $d: A^{2} \rightarrow\{0, \ldots, p-1\} \cup \infty$ be the following mapping:

$$
d(x, y)= \begin{cases}k, & \text { if } x^{\pi^{k}}=y, \\ \infty, & \text { otherwise }\end{cases}
$$

We define

$$
\operatorname{type}_{\varrho}(\bar{a})=\left\{\left(i, j, d\left(a_{i}, a_{j}\right)\right) \mid i, j \in\{1, \ldots, m\} \wedge d\left(a_{i}, a_{j}\right)<\infty\right\} \text {. }
$$

For $\bar{a}, \bar{b} \in A^{m}$ we write type ${ }_{\varrho}(\bar{a}) \sqsubseteq \operatorname{type}_{\varrho}(\bar{b})$ if $\operatorname{type}_{\varrho}(\bar{a}) \subseteq$ type $_{\varrho}(\bar{b})$.

Proposition. The function type $e_{\varrho}$ is a type-function for the relation $\varrho$ and the integer $m$.

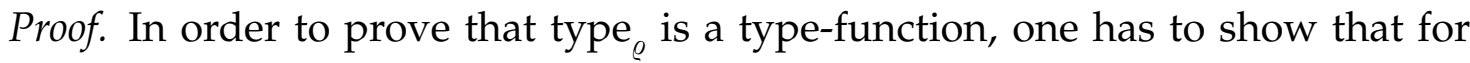
every $\sigma \subseteq A^{m}$ holds $\sigma \in(\operatorname{Inv} \text { End }\{\varrho\})^{(m)}$ if and only if $1.1 .1(1)$ holds.

$(\Leftarrow)$ Let $1.1 .1(1)$ hold. Take $f \in \operatorname{End}\{\varrho\}$ and $\bar{a} \in \sigma$. We define $\bar{b}=f(\bar{a})$, i.e. $b_{i}=f\left(a_{i}\right), i \in\{1, \ldots, m\}$. We will show that $\bar{b} \in \sigma$, i.e. that type ${ }_{\varrho}(\bar{a}) \sqsubseteq \operatorname{type}_{\varrho}(\bar{b})$.

Let $(i, j, k)=\left(i, j, d\left(a_{i}, a_{j}\right)\right) \in$ type $_{\varrho}(\bar{a})$. Then $a_{i}^{\pi^{k}}=a_{j}$. It follows that

$$
f\left(a_{i}^{\pi^{k}}\right)=f\left(a_{j}\right) \Rightarrow\left(f\left(a_{i}\right)\right)^{\pi^{k}}=f\left(a_{j}\right) \Rightarrow b_{i}^{\pi^{k}}=b_{j} \Rightarrow(i, j, k) \in \operatorname{type}_{\varrho}(\bar{b}) .
$$

$(\Rightarrow)$ Let End $\{\varrho\} \subseteq$ End $\{\sigma\}$. Take $\bar{a} \in \sigma$ and $\bar{b} \in A^{m}$ such that type $_{\rho}(\bar{a}) \sqsubseteq \operatorname{type}_{\rho}(\bar{b})$. Further, define the mapping $f$ in the following way:

$$
f(x)= \begin{cases}b_{i}^{\pi^{l}}, & \text { if } x=a_{i}^{\pi^{l}}, l \in\{0, \ldots, p-1\} \\ x, & \text { otherwise }\end{cases}
$$

It is easy to see that $f$ takes $\bar{a}$ to $\bar{b}$.

$f$ is well defined: Let $l_{1} \geq l_{2}$ and let $a_{i}^{\pi^{l_{1}}}=a_{j}^{\pi^{l_{2}}}$. Then

$$
a_{i}^{\pi^{l_{1}-l_{2}}}=a_{j} \Rightarrow d\left(a_{i}, a_{j}\right)=l_{1}-l_{2} \Rightarrow d\left(b_{i}, b_{j}\right)=l_{1}-l_{2} \Rightarrow b_{i}^{\pi^{l_{1}-l_{2}}}=b_{j}
$$

so $f\left(a_{i}^{\pi^{l_{1}}}\right)=b_{i}^{\pi^{l_{1}}}=b_{j}^{\pi^{l_{2}}}=f\left(a_{j}^{\pi^{l_{2}}}\right)$.

$f$ is an endomorphism of $\varrho$ : Let $\left(x, x^{\pi}\right) \in \varrho$. If $x=a_{i}^{\pi^{l}}$, then

$$
\left(f(x), f\left(x^{\pi}\right)\right)=\left(b_{i}^{\pi^{l}}, f\left(a_{i}^{\pi^{l+1}}\right)\right)=\left(b_{i}^{\pi^{l}}, b_{i}^{\pi^{l+1}}\right)=\left(b_{i}^{\pi^{l}},\left(b_{i}^{\pi^{l}}\right)^{\pi}\right) \in \varrho .
$$

Otherwise, $\left(f(x), f\left(x^{\pi}\right)\right)=\left(x, x^{\pi}\right) \in \varrho$.

Hence, $f \in \operatorname{End}\{\sigma\}$ and $\bar{b} \in \sigma$. 
1.2.5. Affine relations. For a binary operation + on $A$ let

$$
\lambda_{+}:=\left\{(x, y, u, v) \in A^{4} \mid x+y=u+v\right\} .
$$

A relation $\varrho$ is called affine if there is an elementary abelian $p$-group $\langle A,+,-, 0\rangle$ on $A$ such that $\varrho=\lambda_{+}$.

Suppose now that $(A,+,-, 0)$ is an elementary abelian $p$-group. It is wellknown that $f \in \operatorname{End}\left\{\lambda_{+}\right\}$if and only if

$$
f(x+y)=f(x)+f(y)-f(0)
$$

for every $x, y \in A$, and we say that $f$ is an affine mapping.

Remark 1. An elementary abelian $p$-group of order $p^{k}$ can be considered as the additive group of a $k$-dimensional vector space over $G F(p)$, and from now on, we will consider elements of $A$ as the vectors of a such vector space.

Lemma. Every affine mapping $f$ on $A$ is of the form

$$
f(\vec{x})=g(\vec{x})+\vec{v}
$$

where $g$ is a linear function and $\vec{v} \in A$. Moreover, for every $\vec{u} \in A$ holds

$$
f(\vec{x})=g(\vec{x}-\vec{u})+f(\vec{u}) .
$$

Corollary. For $\vec{a}_{1}, \ldots, \vec{a}_{m}, \vec{b}_{1}, \ldots, \vec{b}_{m} \in A$ holds that $\left(\vec{a}_{1}, \ldots, \vec{a}_{m}\right)$ can be affine mapped to $\left(\vec{b}_{1}, \ldots, \vec{b}_{m}\right)$ if and only if $\left(\vec{a}_{2}-\vec{a}_{1}, \ldots, \vec{a}_{m}-\vec{a}_{1}\right)$ can be linearly mapped to $\left(\vec{b}_{2}-\vec{b}_{1}, \ldots, \vec{b}_{m}-\right.$ $\left.\vec{b}_{1}\right)$.

Let $\left(\vec{a}_{1}, \ldots, \vec{a}_{m}\right) \in A^{m}$ and let $l$ be the dimension of $\operatorname{Span}\left\{\vec{a}_{1}, \ldots, \vec{a}_{m}\right\}$. Let, further,

$$
S_{\vec{a}_{1}, \ldots, \vec{a}_{m}}:=\left\{\left(i_{1}, \ldots, i_{l}\right) \mid\left(\vec{a}_{i_{1}}, \ldots, \vec{a}_{i_{l}}\right) \text { is a basis of } \operatorname{Span}\left\{\vec{a}_{1}, \ldots, \vec{a}_{m}\right\}, i_{1}<\cdots<i_{l}\right\} \text {. }
$$

We say that $\left(i_{1}, \ldots, i_{l}\right)$ is lexicographically smaller than $\left(j_{1}, \ldots, j_{l}\right)$, and write $\left(i_{1}, \ldots, i_{l}\right)<\left(j_{1}, \ldots, j_{l}\right)$, if $\left(i_{1}, \ldots, i_{l}\right) \neq\left(j_{1}, \ldots, j_{l}\right)$ and for $k_{0}:=\min \{k \mid k \in\{1, \ldots, l\} \wedge$ $\left.i_{k} \neq j_{k}\right\}$ we have that $i_{k_{0}}<j_{k_{0}}$.

Let $\left(i_{1}, \ldots, i_{l}\right)$ be the lexicographically smallest element of $S_{\vec{a}_{1}, \ldots, \vec{a}_{m}}$. Then every $\vec{a}_{i} \in\left\{\vec{a}_{1}, \ldots, \vec{a}_{m}\right\}$ can be uniquely represented as a linear combination:

$$
\vec{a}_{i}=\sum_{j=1}^{l} \alpha_{j i} \vec{a}_{i_{j}} .
$$

For every $\left(\vec{a}_{1}, \ldots, \vec{a}_{m}\right) \in A$ we define its linear type as

$$
\text { type }_{+}\left(\vec{a}_{1}, \ldots, \vec{a}_{m}\right):=\left[\alpha_{j i}\right]_{l \times m} .
$$

Note that $\left[\alpha_{j i}\right]_{l \times m}$ is a matrix of the shape 


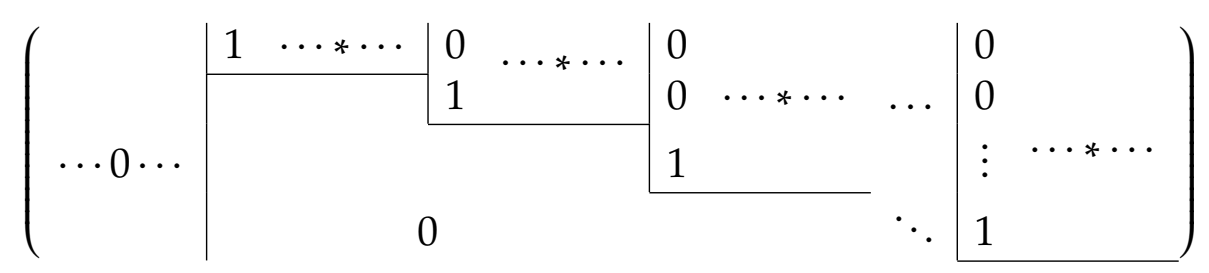

and that every matrix of this shape occurs as a type. Note that special columns (those that begin a block) correspond to the vectors $a_{i_{1}}, \ldots, a_{i_{l}}$. Also, the first block (containing just 0 's) does not necessarily appear.

In the next step we introduce the order on linear types.

Let $\left(\vec{a}_{1}, \ldots, \vec{a}_{m}\right),\left(\vec{b}_{1}, \ldots, \vec{b}_{m}\right) \in A^{m}$, let $\left(i_{1}, \ldots, i_{l}\right)$ be the lexicographically smallest element of $S_{\vec{a}_{1}, \ldots, \vec{a}_{m}}$, and let

$$
\begin{aligned}
& \operatorname{type}_{+}\left(\vec{a}_{1}, \ldots, \vec{a}_{m}\right):=\left[\alpha_{j i}\right]_{l \times m}, \\
& \text { type }_{+}\left(\vec{b}_{1}, \ldots, \vec{b}_{m}\right):=\left[\beta_{j i}\right]_{s \times m} .
\end{aligned}
$$

Let

$$
\left[\gamma_{j k}\right]_{s \times l}:=\left[\beta_{j i_{k}}\right]_{j \in\{1, \ldots, s\}, k \in\{1, \ldots, l\}} .
$$

Then type ${ }_{+}\left(\vec{a}_{1}, \ldots, \vec{a}_{m}\right) \sqsubseteq$ type $_{+}\left(\vec{b}_{1}, \ldots, \vec{b}_{m}\right)$ if

$$
\left[\gamma_{j k}\right]_{s \times l} \cdot\left[\alpha_{j i}\right]_{l \times m}=\left[\beta_{j i}\right]_{s \times m} .
$$

Once we have introduced linear types and linear type-ordering, and having in mind the previous Corollary, we are ready to define affine types and their ordering:

Let $\left(\vec{a}_{1}, \ldots, \vec{a}_{m}\right) \in A^{m}$. Then its affine type is given by

$$
\text { type }_{\lambda_{+}}\left(\vec{a}_{1}, \ldots, \vec{a}_{m}\right):=\text { type }_{+}\left(\vec{a}_{2}-\vec{a}_{1}, \ldots, \vec{a}_{m}-\vec{a}_{1}\right),
$$

For $\left(\vec{b}_{1}, \ldots, \vec{b}_{m}\right) \in A^{m}$ holds type $\lambda_{\lambda_{+}}\left(\vec{a}_{1}, \ldots, \vec{a}_{m}\right) \sqsubseteq \operatorname{type}_{\lambda_{+}}\left(\vec{b}_{1}, \ldots, \vec{b}_{m}\right)$ if

$$
\text { type }_{+}\left(\vec{a}_{2}-\vec{a}_{1}, \ldots, \vec{a}_{m}-\vec{a}_{1}\right) \sqsubseteq \operatorname{type}_{+}\left(\vec{b}_{2}-\vec{b}_{1}, \ldots, \vec{b}_{m}-\vec{b}_{1}\right)
$$

Proposition. The function type ${\lambda_{+}}$is a type-function for the relation $\lambda_{+}$and the integer $m$.

Proof. In order to prove that type ${ }_{0}$ is a type-function, one has to show that for every $\sigma \subseteq A^{m}$ holds $\sigma \in(\operatorname{Inv} \text { End }\{\varrho\})^{(m)}$ if and only if 1.1.1(1) holds.

$(\Leftarrow)$ Let 1.1.1(1) hold. Take $f \in \operatorname{End}\{\varrho\},\left(\vec{a}_{1}, \ldots, \vec{a}_{m}\right) \in \sigma$ and define $\vec{b}_{i}:=f\left(\vec{a}_{i}\right)$. We have to show that type $\lambda_{+}\left(\vec{a}_{1}, \ldots, \vec{a}_{m}\right) \sqsubseteq$ type $_{\lambda_{+}}\left(\vec{b}_{1}, \ldots, \vec{b}_{m}\right)$, which is equivalent to type ${ }_{+}\left(\vec{a}_{2}-\vec{a}_{1}, \ldots, \vec{a}_{m}-\vec{a}_{1}\right) \sqsubseteq$ type $_{+}\left(\vec{b}_{2}-\vec{b}_{1}, \ldots, \vec{b}_{m}-\vec{b}_{1}\right)$..

Let type ${ }_{+}\left(\vec{a}_{2}-\vec{a}_{1}, \ldots, \vec{a}_{m}-\vec{a}_{1}\right)=\left[\alpha_{j i}\right]_{l \times(m-1)}$, and let $\left(i_{1}, \ldots i_{l}\right)$ be the lexicographically smallest element of $S_{\vec{a}_{2}-\vec{a}_{1}, \ldots, \vec{a}_{m}-\vec{a}_{1}}$. Let, further, type $\left.\vec{b}_{+}-\vec{b}_{1}, \ldots, \vec{b}_{m}-\vec{b}_{1}\right)=$ 
$\left[\beta_{j i}\right]_{s \times(m-1)}$, and let $g$ be the linear mapping corresponding to $f$ according to the Lemma above. Take the isomorphisms $\iota_{a}: \operatorname{Span}\left\{\vec{a}_{2}-\vec{a}_{1}, \ldots, \vec{a}_{m}-\vec{a}_{1}\right\} \rightarrow \mathrm{GF}(p)^{l}$ and $\iota_{b}: \operatorname{Span}\left\{\vec{b}_{2}-\vec{b}_{1}, \ldots, \vec{b}_{m}-\vec{b}_{1}\right\} \rightarrow \operatorname{GF}(p)^{s}$ such that

$$
\iota_{a}\left(\vec{a}_{i}-\vec{a}_{1}\right)=\left(\begin{array}{c}
\alpha_{1, i-1} \\
\alpha_{2, i-1} \\
\vdots \\
\alpha_{l, i-1}
\end{array}\right) \quad \text { and } \quad \iota_{b}\left(\vec{b}_{i}-\vec{b}_{1}\right)=\left(\begin{array}{c}
\beta_{1, i-1} \\
\beta_{2, i-1} \\
\vdots \\
\beta_{s, i-1}
\end{array}\right)
$$

We continue by defining a new mapping $\gamma:=\iota_{b} \circ g \circ \iota_{a}^{-1}$ (cf. the diagram below).

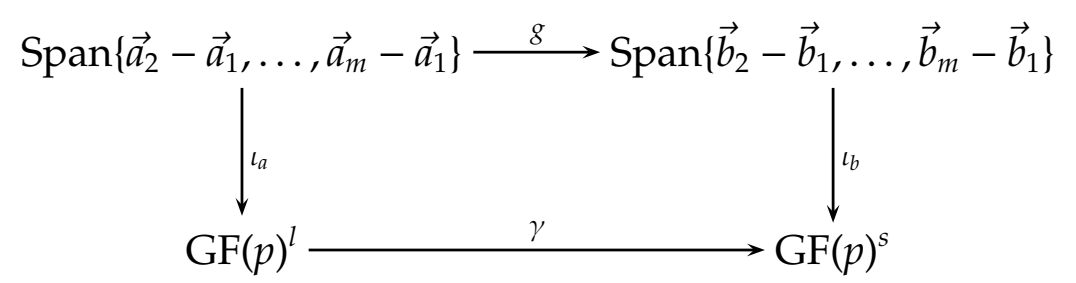

Let $\left[\gamma_{j k}\right]_{s \times l}$ be the matrix of $\gamma$ corresponding to the standard bases of GF $(p)^{l}$ and $\mathrm{GF}(p)^{s}$. Then showing that $\left[\gamma_{j k}\right] \cdot\left[\alpha_{j i}\right]=\left[\beta_{j i}\right]$ is equivalent to showing that

$$
\gamma\left(\begin{array}{c}
\alpha_{1, i-1} \\
\alpha_{2, i-1} \\
\vdots \\
\alpha_{l, i-1}
\end{array}\right)=\left(\begin{array}{c}
\beta_{1, i-1} \\
\beta_{2, i-1} \\
\vdots \\
\beta_{s, i-1}
\end{array}\right)
$$

which is true, since

$$
\gamma\left(\begin{array}{c}
\alpha_{1, i-1} \\
\alpha_{2, i-1} \\
\vdots \\
\alpha_{l, i-1}
\end{array}\right)=\iota_{b} \circ g \circ \iota_{a}^{-1}\left(\begin{array}{c}
\alpha_{1, i-1} \\
\alpha_{2, i-1} \\
\vdots \\
\alpha_{l, i-1}
\end{array}\right)=\iota_{b} \circ g\left(\vec{a}_{i}-\vec{a}_{1}\right)=\iota_{b}\left(\vec{b}_{i}-\vec{b}_{1}\right)=\left(\begin{array}{c}
\beta_{1, i-1} \\
\beta_{2, i-1} \\
\vdots \\
\beta_{s, i-1}
\end{array}\right) .
$$

In particular, from the above follows that the matrix $\left[\gamma_{j k}\right]_{s \times l}$ is of the desired shape as described in (1),

$(\Rightarrow)$ Let End $\{\varrho\} \subseteq$ End $\{\sigma\}$, and let $\left(\vec{a}_{1}, \ldots, \vec{a}_{m}\right) \in \sigma,\left(\vec{b}_{1}, \ldots, \vec{b}_{m}\right) \in A^{m}$ be such that type $_{\lambda_{+}}\left(\vec{a}_{1}, \ldots, \vec{a}_{m}\right) \sqsubseteq \operatorname{type}_{\lambda_{+}}\left(\vec{b}_{1}, \ldots, \vec{b}_{m}\right)$. This means that

$$
\text { type }_{+}\left(\vec{a}_{2}-\vec{a}_{1}, \ldots, \vec{a}_{m}-\vec{a}_{1}\right) \sqsubseteq \operatorname{type}_{+}\left(\vec{b}_{2}-\vec{b}_{1}, \ldots, \vec{b}_{m}-\vec{b}_{1}\right) .
$$

Let type ${ }_{+}\left(\vec{a}_{2}-\vec{a}_{1}, \ldots, \vec{a}_{m}-\vec{a}_{1}\right)=\left[\alpha_{j i}\right]_{l \times(m-1)}$ and let type $\left(\vec{b}_{2}-\vec{b}_{1}, \ldots, \vec{b}_{m}-\vec{b}_{1}\right)=$ $\left[\beta_{j i}\right]_{s \times(m-1)}$. Then (2) means that for the lexicographically smallest tuple $\left(i_{1}, \ldots, i_{k}\right)$ in $S_{\vec{a}_{2}-\vec{a}_{1}, \ldots, \vec{a}_{m}-\vec{a}_{1}}$ holds

$$
\left[\beta_{j i_{k}}\right]_{k \times(m-1)} \cdot\left[\alpha_{j i}\right]_{l \times(m-1)}=\left[\beta_{j i}\right]_{s \times(m-1)} .
$$


Then $\left[\beta_{j i_{k}}\right]_{k \times(m-1)}$ defines a linear mapping $\gamma: \mathrm{GF}(p)^{l} \rightarrow \mathrm{GF}(p)^{s}$. Take the same $\iota_{a}$ and $\iota_{b}$ as above and define

$$
g:=\iota_{b}^{-1} \circ \gamma \circ \iota_{a}
$$

Clearly, $g\left(\vec{a}_{i}-\vec{a}_{1}\right)=\vec{b}_{i}-\vec{b}_{1}$. We define now $f$ to be the following mapping:

$$
f(\vec{x})=g\left(\vec{x}-\vec{a}_{1}\right)+\vec{b}_{1}
$$

It is easy to see that then $f(\vec{x})=g(\vec{x})+\vec{b}_{1}-g\left(\vec{a}_{1}\right)$, so $f$ is affine and, hence, $f \in \operatorname{End}\{\varrho\} \subseteq \operatorname{End}\{\sigma\}$. Note that

$$
f\left(\vec{a}_{i}\right)=g\left(\vec{a}_{i}-\vec{a}_{1}\right)+\vec{b}_{1}=\vec{b}_{i}-\vec{b}_{1}+\vec{b}_{1}=\vec{b}_{i},
$$

so $\left(\vec{b}_{1}, \ldots, \vec{b}_{m}\right) \in \sigma$, which ends the proof.

1.2.6. $h$-regular relations. Let $\Theta=\left\{\theta_{1}, \ldots, \theta_{m}\right\}$ be a family of equivalence relations. We say that $\Theta$ is an $h$-regular family if every $\theta_{i}$ has precisely $h$ blocks, and additionally, if $B_{i}$ is an arbitrary block of $\theta_{i}$ for $i \in\{1, \ldots, m\}$, then $\bigcap_{i=1}^{m} B_{i} \neq \emptyset$.

An $h$-ary relation $\varrho \neq A^{h}$ is $h$-regular if $h \geq 3$ and if there is an $h$-regular family $\Theta$ such that $\left(x_{1}, \ldots, x_{h}\right) \in \varrho$ if and only if for all $\theta \in \Theta$ there are distinct $i, j$ with $\left(x_{i}, x_{j}\right) \in \theta$.

Before concentrating on the relationship between the endomorphism monoids of an $h$-regular and arbitrary other relation, we are going to give some general remarks about $h$-regular relations (see e.g. [40]).

Note that there is another way to define $h$-regular relations. Given a finite set $A,|A| \geq 3$ and an $h$-regular family $\Theta=\left\{\theta_{1}, \ldots, \theta_{m}\right\}$ on the set $A$, let

$$
R_{\Theta}=\left\{\left(x_{1}, \ldots, x_{h}\right) \mid(\forall \theta \in \Theta)(\exists i \neq j) x_{i} \theta x_{j}\right\}
$$

be the corresponding $h$-regular relation. Now, take the set $\{1, \ldots, h\}^{m}$. We define the elementary $(h, m)$-relation $\Psi_{h, m}$ on this set in the following way:

$$
\Psi_{h, m}=\left\{\left(\left[\begin{array}{c}
a_{1}^{1} \\
\vdots \\
a_{m}^{1}
\end{array}\right], \ldots,\left[\begin{array}{c}
a_{1}^{h} \\
\vdots \\
a_{m}^{h}
\end{array}\right]\right) \mid(\forall i \in\{1, \ldots, m\})(\exists j \neq k) a_{i}^{j}=a_{i}^{k}\right\} .
$$

The elementary $(h, m)$-relation is the $h$-regular relation on the set $\{1, \ldots, h\}^{m}$ defined by the $h$-regular family $\Theta^{*}=\left\{\theta_{1}^{*}, \ldots, \theta_{m}^{*}\right\}$, where

$$
\theta_{i}^{*}=\left\{\left(\left[\begin{array}{c}
b_{1}^{1} \\
\vdots \\
b_{m}^{1}
\end{array}\right],\left[\begin{array}{c}
b_{1}^{2} \\
\vdots \\
b_{m}^{2}
\end{array}\right]\right) \mid b_{i}^{1}=b_{i}^{2}\right\}
$$

Note that there exists a surjective mapping $\lambda: A \rightarrow\{1, \ldots, h\}^{m}$ such that 


$$
R_{\Theta}=\left\{\left(x_{1}, \ldots, x_{h}\right) \mid\left(\lambda\left(x_{1}\right), \ldots, \lambda\left(x_{h}\right)\right) \in \Psi_{h, m}\right\} .
$$

The complete characterization of all mappings that preserve $h$-regular relations can be found in [25]. We present here this result specialized for endomorphisms.

Denote by $\bar{x}^{(i)}$ the $i$-th coordinate of the vector $\bar{x}$. Let $f$ be a unary function on the set $A$. We define the function $f_{i}^{\prime}: A \rightarrow\{1, \ldots, h\}$ in the following way:

$$
f_{i}^{\prime}(x):=(\lambda(f(x)))^{(i)} .
$$

Now we can give the characterization:

Proposition 1 ([25]). A unary function $f$ on a set A preserves an $h$-regular relation $R_{\Theta}$ if and only if for each function $f_{i}^{\prime}$ either $f_{i}^{\prime}$ has at most $h-1$ distinct values or there exist a permutation s on $\{1, \ldots, h\}$, and $a v \in\{1, \ldots, m\}$ such that

$$
f_{i}^{\prime}(x):=s\left((\lambda(x))^{(v)}\right)
$$

Before defining type-functions for $h$-regular relations we have to introduce some more notions.

Denote by $\lambda_{\varrho}(\bar{a})^{(i)}=\left(a_{i}^{1}, \ldots, a_{i}^{k}\right)$ the $i$-th row of the matrix presenting the $\bar{a} \in A^{k}$ in $\{1, \ldots, h\}^{m \times k}$.

We define

$$
\begin{aligned}
\mu_{\varrho}(\bar{a})^{(i)} & :=\left\{\left(l_{1}, l_{2}\right) \mid a_{i}^{l_{1}}=a_{i}^{l_{2}}\right\}, \\
I_{\bar{a}} & :=\left\{i \mid 1 \leq i \leq m \text { and }\left\{a_{i}^{1}, \ldots, a_{i}^{k}\right\}=\{1, \ldots, h\}\right\} .
\end{aligned}
$$

Note that $\mu_{\varrho}(\bar{a})^{(i)}$ is an equivalence relation on a set $\{1, \ldots, h\}$. It has $h$ classes if and only if $i \in I_{\bar{a}}$.

Let $\varrho$ be an $h$-regular relation. Then for $\bar{a}=\left(a_{1}, \ldots, a_{k}\right) \in A^{k}$ we define

$$
\begin{aligned}
\tau_{1}(\bar{a}) & =\left\{\mu_{\varrho}(\bar{a})^{(i)} \mid i \in I_{\bar{a}}\right\}, \\
\tau_{2}(\bar{a}) & =\left\{(i, j) \mid i, j \in\{1, \ldots, k\} \wedge a_{i}=a_{j}\right\}, \\
\operatorname{type}_{\varrho}(\bar{a}) & =\left(\tau_{1}(\bar{a}), \tau_{2}(\bar{a})\right) .
\end{aligned}
$$

For $\bar{a}, \bar{b} \in A^{k}$ we write type $e_{\varrho}(\bar{a}) \sqsubseteq \operatorname{type}_{\varrho}(\bar{b})$ if $\tau_{1}(\bar{a}) \supseteq \tau_{1}(\bar{b})$ and $\tau_{2}(\bar{a}) \subseteq \tau_{2}(\bar{b})$.

Proposition 2. The function type $e_{\varrho}$ is a type-function for the relation $\varrho$ and the integer $k$.

Proof. In order to prove that type ${ }_{\varrho}$ is a type-function, one has to show that for every $\sigma \subseteq A^{m}$ holds $\sigma \in(\operatorname{Inv} \operatorname{End}\{\varrho\})^{(m)}$ if and only if 1.1.1(1) holds.

$(\Rightarrow)$ Suppose that End $\{\varrho\} \subseteq$ End $\{\sigma\}$. We need to show that condition 1.1.1(1) holds.

Let $\bar{a}=\left(a_{1}, \ldots, a_{k}\right) \in \sigma, \bar{b}=\left(b_{1}, \ldots, b_{k}\right) \in A^{k}$ be such that type ${ }_{\varrho}(\bar{a}) \sqsubseteq$ type $_{\varrho}(\bar{b})$, i.e.

$$
\left\{\mu_{\varrho}(\bar{b})^{(i)} \mid i \in I_{\bar{b}}\right\} \subseteq\left\{\mu_{\varrho}(\bar{a})^{(i)} \mid i \in I_{\bar{a}}\right\} \text { and }
$$




$$
\forall i, j: a_{i}=a_{j} \Rightarrow b_{i}=b_{j} .
$$

We will construct an endomorphism of $h$-regular relation $\varrho$ that takes $\bar{a}$ to $\bar{b}$. Let $f_{1}^{\prime}, \ldots, f_{m}^{\prime}: A \rightarrow\{1, \ldots, h\}$ be the mappings defined in the following way:

- for $j \in\{1, \ldots, k\}, f_{i}^{\prime}\left(a_{j}\right):=b_{i}^{j}$;

- for $x \in A \backslash\left\{a_{1}, \ldots, a_{k}\right\}$ and $i \notin I_{\bar{b}}, f_{i}^{\prime}(x):=b_{1}^{i}$;

- for $x \in A \backslash\left\{a_{1}, \ldots, a_{k}\right\}$ and for $i \in I_{\bar{b}}$, by $(1)$, there exists a $v \in I_{\bar{a}}$ such that $\mu_{\varrho}(\bar{b})^{(i)}=\tau_{\varrho}(\bar{a})^{(v)}$, so there is a permutation $s$ on $\{1, \ldots, h\}$, such that $s\left(a_{v}^{j}\right)=b_{i}^{j}$, $j \in\{1, \ldots, k\}$. We take $f_{i}^{\prime}(x):=s\left(\lambda(x)^{(v)}\right)$.

Now, for each $x \in A$, let $y(x) \in A$ be any element such that

$$
\lambda(y(x))=\left[\begin{array}{c}
f_{1}^{\prime}(x) \\
\vdots \\
f_{m}^{\prime}(x)
\end{array}\right]
$$

Then we construct

$$
f(x)=\left\{\begin{aligned}
b_{i}, & \text { if } x=a_{i}, \text { for } i \in\{1, \ldots, k\} \\
y(x), & \text { otherwise, }
\end{aligned}\right.
$$

The given mapping $f$ is well-defined because of (2), Moreover, by Proposition 1 , $f \in$ End $\{\varrho\}$, and, therefore, $f \in \operatorname{End}\{\sigma\}$. Since $f(\bar{a})=\left(f\left(a_{1}\right), \ldots, f\left(a_{k}\right)\right)=\left(b_{1}, \ldots, b_{k}\right)=$ $\bar{b}$, it follows $\bar{b} \in \sigma$.

$(\Leftarrow)$ Let condition 1.1.1(1) hold. We will show that $\operatorname{End}\{\varrho\} \subseteq \operatorname{End}\{\sigma\}$.

Let $g \in$ End $\{\varrho\}, \bar{a}=\left(a_{1}, \ldots, a_{k}\right) \in \sigma$, and $b_{i}:=g\left(a_{i}\right), i \in\{1, \ldots, k\}$ (i.e. $\bar{b}:=$ $\left.\left(b_{1}, \ldots, b_{k}\right)=g(\bar{a})\right)$. We want to show that $\bar{b} \in \sigma$

Since 1.1.1(1) holds, it is enough to show that type $(\bar{b}) \sqsubseteq \operatorname{type}_{\varrho}(\bar{a})$, i.e. that $(1)$ and (2) hold. Here (2) holds because $\bar{b}=g(\bar{a})$, so it remains to show (1).

By assumption, $g$ is an endomorphism of the $h$-regular relation $\varrho$, so it fulfills the conditions given in Proposition 1. If $g_{i}^{\prime}$ has less then $h$ images, then $i \notin I_{\bar{b}}$. Otherwise, there exist a permutation $s$ on $\{1, \ldots, h\}$ and a $v \in\{1, \ldots, m\}$ such that $g_{i}^{\prime}(x)=s\left(\lambda(x)^{(v)}\right)$.

Specially, for $\bar{a}=\left(a_{1}, \ldots, a_{k}\right)$ we have

$$
b_{i}^{j}=g_{i}^{\prime}\left(a_{j}\right)=s\left(a_{v}^{j}\right),
$$

and, therefore, for $i \in I_{\bar{b}}$,

$$
\begin{aligned}
\mu_{\varrho}(\bar{b})^{(i)} & =\left\{\left(l_{1}, l_{2}\right) \mid b_{i}^{l_{1}}=b_{i}^{l_{2}}\right\}=\left\{\left(l_{1}, l_{2}\right) \mid s\left(a_{v}^{l_{1}}\right)=s\left(a_{v}^{l_{2}}\right)\right\}= \\
& =\left\{\left(l_{1}, l_{2}\right) \mid a_{v}^{l_{1}}=a_{v}^{l_{2}}\right\}=\mu_{\varrho}(\bar{a})^{(v)} .
\end{aligned}
$$

Hence, for every $i \in I_{\bar{b}}$ there exists a $v \in I_{\bar{a}}$ such that $\mu_{\varrho}(\bar{b})^{(i)}=\mu_{\varrho}(\bar{a})^{(v)}$. Therefore (1) holds. 


\subsection{Applications of local methods (Examples)}

Example 1. The goal of this example is to demonstrate the use of local methods for the constructive enumeration of relations in the $m$-ary part of a given weak Krasner algebra. In particular, we will enumerate all relations in the ternary part of the weak Krasner algebra of three-element chain. This problem in itself is not very interesting, but it should give the reader a good idea how such problems can be solved in general using local methods and, moreover, it is the smallest nontrivial problem that can be completely and feasibly handled without the use of computer.

Let $\varrho$ be a linear order on $A=\{0,1, x\}$, where 0 is the smallest and 1 is the greatest element. We want to describe a lattice of all ternary relations $\sigma$ in Inv End $\{\varrho\}$. The first step is to describe types and their order. Since $\varrho$ is a bounded lattice, types are given by 1.2 .3 , so they are of the shape $\left(\tau_{1}, \tau_{2}\right)$, where $\tau_{1}$ is a linear quasi-order and $\tau_{2}=\tau_{1} \cap \tau_{1}{ }^{-1}$, so in this particular case, types depend just on $\tau_{1}$. Therefore, type-order is simply the natural inclusion-order on linear quasi-order relations on the set $\{1,2,3\}$. This poset of linear quasi-orders is given in (1).

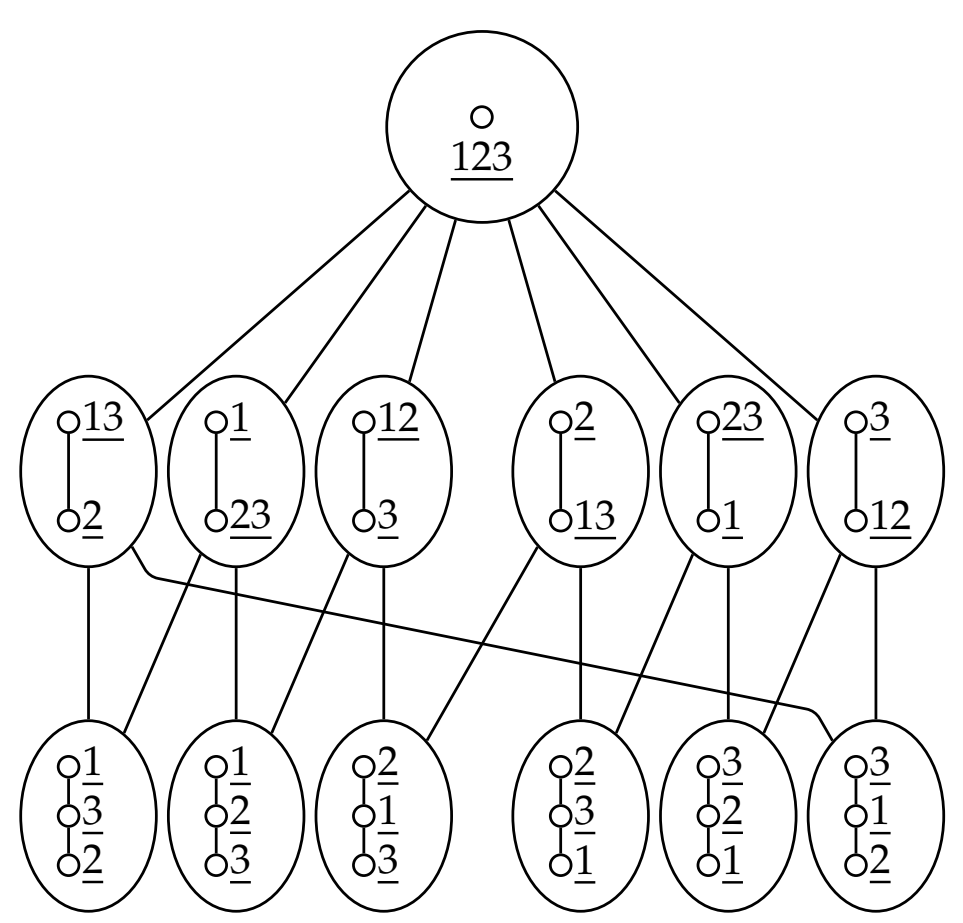

The number of ternary relations $\sigma$ is equal then to the number of antichains in the given poset and we give it in the following table: 


\begin{tabular}{|c|c|}
\hline length & number of antichains \\
\hline 0 & 1 \\
\hline 1 & 13 \\
\hline 2 & 54 \\
\hline 3 & 112 \\
\hline 4 & 105 \\
\hline 5 & 36 \\
\hline 6 & 2 \\
\hline
\end{tabular}

So, all together, there are 323 distinct ternary relations in the weak Krasner algebra generated by $\varrho$.

Example 2. The goal of this example is to describe an $h$-regular relation $\varrho$ and a central relation $\sigma$ over the same basic set such that End $\{\varrho\} \subset$ End $\{\sigma\}$. Let $A=\left\{a_{1}, a_{2}, \ldots, a_{10}\right\}$ and let $\varrho$ be the 3-regular relation defined by the 3-regular family $\Theta=\left\{\theta_{1}, \theta_{2}\right\}$, where

$$
\begin{aligned}
& \theta_{1}=\left\{\underline{a_{1}, a_{2}, a_{3}, a_{4}}, \underline{a_{5}, a_{6}, a_{7}}, \underline{a_{8}, a_{9}, a_{10}}\right\} \\
& \theta_{2}=\underline{a_{1}, a_{2}, a_{5}, a_{8}}, \underline{a_{3}, a_{6}, a_{9}}, \underline{\underline{a_{4}, a_{7}, a_{10}}} .
\end{aligned}
$$

Note that the equivalence relations from the 3-regular family are not given as sets of ordered pairs, but as the partitions of the set $A$.

The representation of the elements of $A$ is then given by the following table:

\begin{tabular}{c|c|c|c|c|c|c|c|c|c|c}
$x$ & $a_{1}$ & $a_{2}$ & $a_{3}$ & $a_{4}$ & $a_{5}$ & $a_{6}$ & $a_{7}$ & $a_{8}$ & $a_{9}$ & $a_{10}$ \\
\hline$\lambda(x)$ & {$\left[\begin{array}{l}1 \\
1\end{array}\right]$} & {$\left[\begin{array}{l}1 \\
1\end{array}\right]$} & {$\left[\begin{array}{l}1 \\
2\end{array}\right]$} & {$\left[\begin{array}{l}1 \\
3\end{array}\right]$} & {$\left[\begin{array}{l}2 \\
1\end{array}\right]$} & {$\left[\begin{array}{l}2 \\
2\end{array}\right]$} & {$\left[\begin{array}{l}2 \\
3\end{array}\right]$} & {$\left[\begin{array}{l}3 \\
1\end{array}\right]$} & {$\left[\begin{array}{l}3 \\
2\end{array}\right]$} & {$\left[\begin{array}{l}3 \\
3\end{array}\right]$}
\end{tabular}

For $\sigma$ we take the smallest central relation of arity 6 with the center $C_{\sigma}=\left\{a_{3}\right\}$ such that End $\{\varrho\} \subseteq$ End $\{\sigma\}$. This relation can be obtained from the smallest central relation $\sigma_{C}$ with the center $a_{3}$ by closing it using the type-function given in 1.2.6 and Condition 1.1.1(1),

It is left to show that the obtained central relation $\sigma$ is not a full relation. For this purpose, take the element $\bar{b}=\left(a_{1}, a_{2}, a_{6}, a_{7}, a_{9}\right)$. It is represented by

$$
\lambda(\bar{b})=\left(\left[\begin{array}{l}
1 \\
1
\end{array}\right],\left[\begin{array}{l}
1 \\
1
\end{array}\right],\left[\begin{array}{l}
2 \\
2
\end{array}\right],\left[\begin{array}{l}
2 \\
3
\end{array}\right],\left[\begin{array}{l}
3 \\
2
\end{array}\right],\left[\begin{array}{l}
3 \\
3
\end{array}\right]\right) .
$$

It follows that $I_{\bar{b}}=\{1,2\}$. The corresponding equivalence relations are given by their partition form:

$$
\begin{aligned}
& \mu_{\varrho}(\vec{b})^{(1)}=\{\underline{1,2}, \underline{3,4}, \underline{5,6\}}, \\
& \mu_{\varrho}(\vec{b})^{(2)}=\{\underline{1,2}, \underline{3,5}, \underline{4,6\}},
\end{aligned}
$$

and they form $\tau_{1}(\bar{b})$. 
Note that it suffices to show that $\tau_{1}(\bar{b}) \nsubseteq \tau_{1}(\bar{a})$ for all irreflexive tuples $\bar{a}$ from $\sigma_{C}$, since inclusion is transitive and $\sigma$ is generated by $\sigma_{C}$, using 1.2.6 and Condition 1.1.1(1),

So, at this point we are looking for an irreflexive tuple $\bar{a}$ that contains $a_{3}$ as an entry and satisfies the condition type $e_{\rho}(\bar{a}) \sqsubseteq$ type $_{\varrho}(\bar{b})$ (i.e. $\tau_{1}(\bar{b}) \subseteq \tau_{1}(\bar{a})$ ), for given $\bar{b}$. In this particular case, that means that $\bar{b}$ and $\bar{a}$ should have equal types and, therefore, the first two entries of $\lambda(\bar{a})$ should be equal. Since $\bar{a}$ is supposed to be irreflexive, the only two candidates for the first two entries of $\bar{a}$ are $a_{1}$ and $a_{2}$. So, if such $\bar{a}$ exists, then it should contain $a_{1}, a_{2}$ and $a_{3}$ and, therefore, the first row of $\lambda(\bar{a})$ contains at least three entries 1. It immediately follows that type $_{\varrho}(\bar{b}) \neq$ type $_{\varrho}(\bar{a})$, so such $\bar{a}$ does not exist.

This construction gives us the example of an $h$-regular relation $\varrho$ and a central relation $\sigma$ such that End $\{\varrho\} \subseteq \operatorname{End}\{\sigma\}$. Moreover, this inclusion is proper, i.e. there exists an $f \in \operatorname{End}\{\sigma\} \backslash$ End $\{\varrho\}$. For instance, one can easily check that

$$
f(x)=\left\{\begin{array}{cl}
a_{3}, & \text { for } x=a_{5} \\
x, & \text { otherwise }
\end{array}\right.
$$

preserves $\sigma$, but not $\varrho$. 



\section{Endolocal relational structures}

In the previous chapter we introduced the notion of type-functions and we have the Locality Principle. The examples that followed made use of different type-functions, where each of them was defined in an ad hoc manner. Our goal now is to study type-functions systematically. In 2.1 we introduce and study a Galois connection between relations and sets of formulae. Section 2.2 is dedicated to $k$-endolocal relational structures and criteria for testing this property using the language of formal concept analysis (i.e. in the terms of a given Galois connection). In 2.3 two approaches to endolocal relational structures will be made: one from the point of view of logics and one from the point of view of models. Additionally, we will establish a connection between endolocality and homomorphism-homogeneity. The information from the first three sections will be collected and presented in a compact and clearly represented manner in 2.4 . Finally, the last section of this chapter is dedicated to the application of the theory of endolocal relational structures in the study of weak Krasner algebras.

\subsection{Towards the notion of endolocality}

In this section we will create the theoretical framework for the use of the Locality Principle, that is, we develop a technique for producing type-functions of different strengths leading to a hierarchy of classes of relational structures according to the corresponding type-function.

2.1.1. A Galois connection between relations and sets of logical formulae. To the notion of endolocality we come with a little help of Formal Concept Analysis. As the starting point, for a given relational structure $\mathbf{A}=\left(A,\left(\varrho_{\mathbf{A}}\right)_{\varrho \in R}\right)$ over the relational signature $R$, we introduce the formal context $\mathbb{K}_{m}(\mathbf{A}):=\left(A^{m}, \Phi_{m}(\exists, \wedge, \vee,=\right.$ $, R), \models)$. Its objects are then $m$-tuples, its attributes are positive existential formulae with $m$ free variables $x_{1}, \ldots, x_{m}$ and the binary relation between objects and attributes is the relation of satisfaction as it was defined in 0.2.1.

The operators that establish a Galois connection between powerset lattices on $A^{m}$ and $\Phi_{m}(\exists, \wedge, \vee,=, R)$ are then given as follows:

For $\sigma \subseteq A^{m}$ and $\Psi \subseteq \Phi_{m}(\exists, \wedge, \vee,=, R)$

$$
\begin{aligned}
\sigma^{\prime} & :=\left\{\varphi \in \Phi_{m}(\exists, \wedge, \vee,=, R) \mid \forall \bar{b} \in \sigma: \bar{b} \models \varphi\right\} \\
\Psi^{\prime} & :=\left\{\bar{a} \in A^{m} \mid \forall \psi \in \Psi: \bar{a} \models \psi\right\} .
\end{aligned}
$$


Further, a pair $(\sigma, \Psi)$ will be a formal concept of $\mathbb{K}_{m}(\mathbf{A})$ with intent $\Psi$ and extent $\sigma$ if and only if for $\sigma \subseteq A^{m}$ and $\Psi \subseteq \Phi_{m}(\exists, \wedge, \vee,=, R)$ holds

$$
\sigma^{\prime}=\Psi \text { and } \sigma=\Psi^{\prime}
$$

A closer look to this definition gives us that every intent $\Psi$ is of the form

$$
\Psi=\left\{\varphi \in \Phi_{m}(\exists, \wedge, \vee,=, R) \mid \forall \bar{b} \in \sigma: \bar{b} \vDash \varphi\right\}=\operatorname{pTh}_{\mathbf{A}}(\sigma),
$$

and every extent $\sigma$ is of the form

$$
\begin{aligned}
\sigma & =\left\{\bar{a} \in A^{m} \mid \forall \psi \in \Psi: \bar{a} \models \psi\right\}=\left\{\bar{a} \in A^{m} \mid \forall \psi \in \Psi: \bar{a} \in \psi^{\mathbf{A}}\right\}= \\
& =\bigcap_{\psi \in \Psi} \psi^{\mathbf{A}}=\bigcap_{\psi \in \operatorname{pTh}_{\mathbf{A}}(\sigma)} \psi^{\mathbf{A}} .
\end{aligned}
$$

2.1.2. The extents of $K_{m}(A)$. At this point we recall that extents and intents are nothing else but Galois-closed sets with respect to the given Galois connection (i.e. $\sigma^{\prime \prime}=\sigma$ and $\Psi^{\prime \prime}=\Psi$ ) and we direct our attention to the $\operatorname{set} \operatorname{Ext}\left(\mathbb{K}_{m}(\mathbf{A})\right.$ ) of all extents of $\mathbb{K}_{m}(\mathbf{A})$.

The set $\operatorname{Ext}\left(\mathbb{K}_{m}(\mathbf{A})\right)$ is a closure system, that is, it is closed with respect to arbitrary intersections and it contains $A^{m}$. Moreover, this particular closure system satisfies even more:

Proposition 1. The closure system of all extents of $\left(A^{m}, \Phi_{m}(\exists, \wedge, \vee,=, R), \models\right)$ is closed with respect to finite unions.

Proof. It is enough to show that if $\sigma_{1}, \sigma_{2} \subseteq A^{m}$ are extents, then $\sigma_{1} \cup \sigma_{2}$ is also an extent. For the proof, define

$$
T:=\left\{\varphi_{1} \vee \varphi_{2} \mid \varphi_{1} \in \sigma_{1}^{\prime}, \varphi_{2} \in \sigma_{2}^{\prime}\right\}
$$

It is not hard to see that $T \subseteq\left(\sigma_{1} \cup \sigma_{2}\right)^{\prime}$. Namely, for arbitrary $\varphi_{1} \vee \varphi_{2} \in T$ we have that $\varphi_{1} \in \sigma_{1}^{\prime}$ and $\varphi_{2} \in \sigma_{2}^{\prime}$. It follows that for every $\bar{b} \in \sigma_{1}$ we have that $\bar{b} \vDash \varphi_{1}$ and for every $\bar{b} \in \sigma_{2}$ we have that $\bar{b} \vDash \varphi_{2}$. From these two facts it follows straightforward that for every $\bar{b} \in \sigma_{1} \cup \sigma_{2}$ it holds that $\bar{b} \vDash \varphi_{1} \vee \varphi_{2}$, so $\varphi_{1} \vee \varphi_{2} \in\left(\sigma_{1} \cup \sigma_{2}\right)^{\prime}$.

This fact we will use to prove that $\left(\sigma_{1} \cup \sigma_{2}\right)^{\prime \prime}=\sigma_{1} \cup \sigma_{2}$. The extensivity of the closure operator " gives immediately $\left(\sigma_{1} \cup \sigma_{2}\right)^{\prime \prime} \supseteq \sigma_{1} \cup \sigma_{2}$. The other inclusion we show indirectly. Suppose there is a $\bar{b} \in\left(\sigma_{1} \cup \sigma_{2}\right)^{\prime \prime} \backslash\left(\sigma_{1} \cup \sigma_{2}\right)$. Then $\bar{b} \notin \sigma_{1}$ and $\bar{b} \notin \sigma_{2}$, so there exists a $\varphi_{1} \in \sigma_{1}^{\prime}$ such that $\bar{b} \not \forall \varphi_{1}$ and there exists a $\varphi_{2} \in \sigma_{2}^{\prime}$ such that $\bar{b} \not \models \varphi_{2}$. This implies that $\bar{b} \not \neq \varphi_{1} \vee \varphi_{2}$. On the other hand, $\varphi_{1} \vee \varphi_{2} \in T \subseteq\left(\sigma_{1} \cup \sigma_{2}\right)^{\prime}$, so it follows that $\bar{b} \notin\left(\sigma_{1} \cup \sigma_{2}\right)^{\prime \prime}$, which is a contradiction.

An immediate consequence of Proposition 1 is that for arbitrary cardinality of the set $A$, the set $\operatorname{Ext}\left(\mathbb{K}_{m}(\mathbf{A})\right)$, ordered by inclusion, forms a distributive lattice. 
Remark. There is one more intriguing insight that should be mentioned at this place, but which will not play a role in the sequel. It requires a little detour to topological spaces. Note that on the set $A^{m}$ the operator "fulfils all Kuratowski closure axioms. That is, for all $Y, Z \subseteq A^{m}$ :

(C1) $Y \subseteq Y^{\prime \prime}$ (Extensivity)

(C2) $\left(Y^{\prime \prime}\right)^{\prime \prime}=Y^{\prime \prime}$ (Idempotence)

(C3) $(Y \cup Z)^{\prime \prime}=Y^{\prime \prime} \cup Z^{\prime \prime}$ (Preservation of binary unions)

(C4) $\emptyset^{\prime \prime}=\emptyset$ (Preservation of nullary unions)

Indeed, the fulfilment of (C1)-(C3) is obvious. To see that (C4) is fulfilled, too, note that

$$
\emptyset^{\prime}=\left\{\varphi \in \Phi_{m}(\exists, \wedge, \vee,=, R) \mid \forall \bar{b} \in \emptyset: \bar{b} \vDash \varphi\right\}=\Phi_{m}(\exists, \wedge, \vee,=, R),
$$

so

$$
\emptyset^{\prime \prime}=\Phi_{m}(\exists, \wedge, \vee,=, R)^{\prime}=\left\{\bar{a} \in A^{m} \mid \forall \psi \in \Phi_{m}(\exists, \wedge, \vee,=, R): \bar{a} \vDash \psi\right\} .
$$

In particular, then for $\bar{a} \in \emptyset^{\prime \prime}$ must hold $\bar{a} \vDash \mathrm{F}$, so $\emptyset^{\prime \prime}=\emptyset$. This is equipping the structure $\mathbf{A}$ and all its finite powers with a natural topology.

We continue the study of the given context by restricting ourself with respect to the cardinality of set $A$.

Recall that Birkhoff's representation theorem for finite distributive lattices states that every finite distributive lattice is isomorphic to the lattice of down-sets of the poset of join-irreducible elements. Hence, for finite basic set $A$, the closure system $\operatorname{Ext}\left(\mathbb{K}_{m}(\mathbf{A})\right)$ is completely described by its join-irreducible elements.

Proposition 2. Every join-irreducible element of $\left(\operatorname{Ext}\left(\mathbb{K}_{m}(\mathbf{A})\right), \subseteq\right)$ is of the form $\{\bar{a}\}^{\prime \prime}$, for some $\bar{a} \in A^{m}$. Moreover, if $A$ is finite, then for every $\bar{a} \in A^{m},\{\bar{a}\}^{\prime \prime}$ is a join-irreducible element.

Proof. Let $\tau$ be a join-irreducible element of $\left(\operatorname{Ext}\left(\mathbb{K}_{m}(\mathbf{A})\right), \subseteq\right)$. Note that $\tau=\bigcup_{\bar{a} \in \tau}\{\bar{a}\}$. On the other hand, $\tau=\tau^{\prime \prime}$, so it follows that

$$
\tau=\tau^{\prime \prime}=\left(\bigcup_{\bar{a} \in \tau}\{\bar{a}\}\right)^{\prime \prime}=\bigcup_{\bar{a} \in \tau}\{\bar{a}\}^{\prime \prime} .
$$

From the join-irreducibility of $\tau$ now directly follows that $\tau=\{\bar{a}\}^{\prime \prime}$, for some $\bar{a} \in \tau$.

Suppose now that $A$ is finite. We know that $\{\bar{a}\}^{\prime \prime}=\left\{\bar{b} \in A^{m} \mid\{\bar{a}\}^{\prime} \subseteq\{\bar{b}\}^{\prime}\right\}$. Define

$$
\tau:=\left\{\bar{b} \in A^{m} \mid\{\bar{a}\}^{\prime} \subset\{\bar{b}\}^{\prime}\right\} .
$$

Note that then $\bar{a} \in\{\bar{a}\}^{\prime \prime} \backslash \tau$, so $\tau \subset\{\bar{a}\}^{\prime \prime}$. We will show that for every $\sigma=\sigma^{\prime \prime}$ such that $\sigma \subset\{\bar{a}\}^{\prime \prime}$ holds $\sigma \subseteq \tau$. 
As it was already observed, $\sigma=\bigcup_{\bar{b} \in \sigma}\{\bar{b}\}^{\prime \prime} \subset\{\bar{a}\}^{\prime \prime}$, so for every $\bar{b} \in \sigma$ holds $\{\bar{b}\}^{\prime \prime} \subset\{\bar{a}\}^{\prime \prime}$. From the properties of Galois connections we obtain that for every $\bar{b} \in \sigma$ holds $\{\bar{b}\}^{\prime}=\{\bar{b}\}^{\prime \prime \prime} \supset\{\bar{a}\}^{\prime \prime \prime}=\{\bar{a}\}^{\prime}$, so $\sigma \subseteq \tau$.

Since $A$ is finite, there are just finitely many closed sets $\sigma$ that are strictly below $\{\bar{a}\}^{\prime \prime}$. All of them are below $\tau$, so their union is a closed set below $\tau$, too, and therefore strictly below $\{\bar{a}\}^{\prime \prime}$.

An other way to describe $\operatorname{Ext}\left(\mathbb{K}_{m}(\mathbf{A})\right)$ is to study its connections to other known closure systems. Here we obtain the following:

Proposition 3. Let $A$ be a finite set. Then the closure system (Inv End $\mathbf{A})^{(m)}$ is equal to $\operatorname{Ext}\left(\mathbb{K}_{m}(\mathbf{A})\right)$.

Proof. We will use the fact that two closure system over the same set are equal if they have the same closure operator. For $B \subseteq A^{m}$ the closure operator on (Inv End $\mathbf{A})^{(m)}$ is given by $\sigma \mapsto \Gamma_{\text {End } \mathbf{A}}(\sigma)$, while the closure operator on extents is $\sigma \mapsto \sigma^{\prime \prime}$. Since $\sigma^{\prime \prime}$ is an extent, it follows that $\sigma^{\prime \prime}=\bigcap_{\psi \in \mathrm{pTh}_{\mathbf{A}}\left(\sigma^{\prime \prime}\right)} \psi^{\mathbf{A}}$. On the other hand, $\operatorname{pTh}_{\mathbf{A}}\left(\sigma^{\prime \prime}\right)=\sigma^{\prime \prime \prime}=\sigma^{\prime}=\operatorname{pTh}_{\mathbf{A}}(\sigma)$, so $\sigma^{\prime \prime}=\bigcap_{\psi \in \operatorname{pTh}_{\mathbf{A}}(\sigma)} \psi^{\mathbf{A}}$. Moreover, $\operatorname{pTh}_{\mathbf{A}}(\sigma)$ is the set of all formulae $\psi$ such that $\sigma \subseteq \psi^{\mathrm{A}}$. A part of the main theorem in clone theory (cf. [40, Hauptsatz 2.1.3.(ii)]) states that all elements from Inv End A are of the shape $\varphi^{\mathbf{A}}$ for some $\varphi \in \Phi(\exists, \wedge, \vee,=, R)$. From this it follows that $\bigcap_{\psi \in \operatorname{pTh}_{\mathbf{A}}(\sigma)} \psi^{\mathbf{A}}=\bigcap\{\varrho \in \operatorname{Inv}$ End $\mathbf{A} \mid \sigma \subseteq \varrho\}=\Gamma_{\text {End } \mathbf{A}}(\sigma)$.

2.1.3. Internal characterization of $\operatorname{Ext}\left(\mathbb{K}_{m}(\mathbf{A})\right)$. Once the connection between these two closure systems is established, the way to the internal characterization of $\operatorname{Ext}\left(\mathbb{K}_{m}(\mathbf{A})\right)$, for finite $A$, is open. Here, under "internal" we understand a description of the closure system $\operatorname{Ext}\left(\mathbb{K}_{m}(\mathbf{A})\right)$ using its implicational theory. First we note that the implications $\{\bar{a}\} \rightarrow\{\bar{b}\}$ for $\{\bar{a}\}^{\prime} \subseteq\{\bar{b}\}^{\prime}$, generally hold in $\operatorname{Ext}\left(\mathbb{K}_{m}(\mathbf{A})\right)$. Moreover, for finite distributive closure systems it is known (cf. [8]) that the implications of this shape generate the implicational theory of the closure system. The following proposition sums this up for our contexts $\mathbb{K}_{m}(\mathbf{A})$. In order to keep this presentation self-contained, we present it together with the proof.

Proposition 1. Let $A$ be a finite set and let $\sigma \subseteq A^{m}$. Then $\sigma=\sigma^{\prime \prime}$ if and only if

$$
\forall \bar{a}, \bar{b} \in A^{m} \quad \bar{a} \in \sigma \wedge \bar{b} \vDash \operatorname{pTh}(\bar{a}) \Rightarrow \bar{b} \in \sigma .
$$

Proof. $(\Rightarrow)$ Let $\sigma=\sigma^{\prime \prime}$, let $\bar{a} \in \sigma$ and let $b \in A^{m}$ such that $\bar{b} \vDash \operatorname{pTh}(\bar{a})$. Then $\{\bar{a}\} \subseteq \sigma$, so $\{\bar{a}\}^{\prime} \supseteq \sigma^{\prime}$. On the other hand, $\bar{b} \vDash \operatorname{pTh}(\bar{a})$ is equivalent to $\{\bar{b}\}^{\prime} \supseteq\{\bar{a}\}^{\prime}$, so $\{\bar{b}\}^{\prime} \supseteq \sigma^{\prime}$. Further we obtain that $\{\bar{b}\}^{\prime \prime} \subseteq \sigma^{\prime \prime}=\sigma$, which combined with the extensivity of the closure operator" gives that $\bar{b} \in \sigma$.

$(\Leftarrow)$ It suffices to show that $\sigma^{\prime \prime} \subseteq \sigma$, since the other inclusion always holds. The finiteness of the set $A$ allows us to write $\sigma$ as the finite union of its elements, i.e. $\sigma=\bigcup_{\bar{a} \in \sigma}\{\bar{a}\}$. 
Let us now look a bit closer onto Condition (1). Take an arbitrary $\bar{a} \in \sigma$. As we already pointed out, $\bar{b} \vDash \operatorname{pTh}(\bar{a})$ is equivalent to $\bar{b} \in\{\bar{a}\}^{\prime \prime}$, so the given condition implies that $\{\bar{a}\}^{\prime \prime} \subseteq \sigma$.

Combining this fact with 2.1.2(Proposition 1), we obtain

$$
\sigma^{\prime \prime}=\left(\bigcup_{\bar{a} \in \sigma}\{\bar{a}\}\right)^{\prime \prime}=\bigcup_{\bar{a} \in \sigma}\{\bar{a}\}^{\prime \prime} \subseteq \sigma .
$$

Remark. Note that the $(\Rightarrow)$ direction of the proof does not depend on the cardinality of $A$.

2.1.4. Another instance of the Locality Principle. Condition 2.1.3(1) provides another example of the Locality Principle. Indeed, as the space we can take the set $A^{m}$. The set of observations is given by the set of all intents of $\mathbb{K}_{m}(\mathbf{A})$ ordered by inclusion. Finally, the observer is the operator $\mathrm{pTh}_{\mathrm{A}}$. By 2.1.3(Proposition 1) we have that the closure system defined by the Locality Principle is equal to the closure system of the extents of the context $\mathbb{K}_{m}(\mathbf{A})$. Moreover, for the finite basic set $A$ this closure system is equal to the closure system $(\operatorname{Inv} \operatorname{End}(\mathbf{A}))^{(m)}$, i.e. the $m$-ary part of the weak Krasner algebra generated by $\mathbf{A}$.

\subsection{Endolocality}

In Chapter 1 we have seen examples of relational structures where a small amount of information was enough to describe a corresponding type-function that by the Locality Principle describes the $m$-ary part of the weak Krasner algebra generated by the structure. In particular, each of the described type-functions required just finitely many facts to be completely defined, while the image of the type-function $\mathrm{pTh}_{\mathrm{A}}$ always contains infinitely many facts about the given structure. For this reason we will look for finite approximations of $\mathrm{pTh}_{\mathrm{A}}$ that are equivalent to the $\mathrm{pTh}_{\mathrm{A}}$ for the given relational structure A. Natural approximations of $\mathrm{pTh}$ for relational structures are the operators $\mathrm{pTh}_{\mathrm{A}}^{(k)}$, for $k \in \mathbb{N}$, i.e. the restrictions of $\mathrm{pTh}_{\mathrm{A}}$ to the formulae of the quantifier depth at most $k$. Moreover, if the signature $R$ is finite, then by 0.2 .2 (Corollary) the images of $\mathrm{pTh}_{\mathrm{A}}^{(k)}$, up to equivalence, contain finitely many formulae.

2.2.1. $k$-endolocality. A relational structure $\mathbf{A}=\left(A,\left(\varrho_{\mathbf{A}}\right)_{\varrho \in R}\right)$ will be called $k$ endolocal if for every $m \in \mathbb{N}$ and every $\sigma \subseteq A^{m}$ holds

$$
\sigma=\sigma^{\prime \prime} \text { if and only if } \forall \bar{a}, \bar{b} \in A^{m}: \bar{a} \in \sigma \wedge \bar{b} \vDash \operatorname{pTh}_{\mathbf{A}}^{(k)}(\bar{a}) \Rightarrow \bar{b} \in \sigma .
$$

If a relational structure is 0 -endolocal, then we simply call it endolocal. Note that if a relational structure is $k$-endolocal, then it is also $(k+1)$-endolocal. As a matter of fact, in Chapter 1 we have already met examples of $k$-endolocal structures. 
Example 1. Recall the definition of a type-function for equivalence relations in 1.2.1. Every observation that is made by the given type-function about an $m$ tuple $\bar{a}$ can be formalized as a set of quantifier-free formulae from $\Phi_{m}(\exists, \wedge, \vee,=, R)$. Indeed, let $\mathbf{A}=\left(A, \varrho_{\mathbf{A}}\right)$ be a relational structure over a relational signature $R=\{\varrho\}$, such that $\varrho_{\mathrm{A}}$ is an equivalence relation over $A$. Then $\tau_{1}(\bar{a})$ can be expressed as

$$
\hat{\tau}_{1}(\bar{a})=\left\{\varrho\left(x_{i}, x_{j}\right) \mid\left(a_{i}, a_{j}\right) \in \varrho_{\mathbf{A}}\right\},
$$

and $\tau_{2}(\bar{a})$ can be expressed as

$$
\hat{\tau}_{2}(\bar{a})=\left\{x_{i}=x_{j} \mid a_{i}=a_{j}\right\} .
$$

It is easy to see that $\hat{\tau}_{i}(\bar{a}) \subseteq \hat{\tau}_{i}(\bar{b})$ if and only if $\tau_{i}(\bar{a}) \subseteq \tau_{i}(\bar{b}), i=1,2$. From this and 1.2.1(Proposition), it follows that $\mathbf{A}$ is a 0 -endolocal, that is, endolocal relational structure.

Example 2. If we recall now the definition of the type-function for permutational relations in 1.2.4, we arrive at a slightly different situation. Here every observation can be formalized in the following way:

Let $\mathbf{A}=\left(A, \varrho_{\mathbf{A}}\right)$ be a relational structure over the relational signature $R=\{\varrho\}$, such that $\varrho_{\mathrm{A}}$ is a permutational relation corresponding to a fix-point free permutation of the prime order $p$ on $A$. For a given $k$ we define

$$
\varphi_{k}\left(x_{i}, x_{j}\right):=\left(\exists y_{1}\right)\left(\exists y_{2}\right) \ldots\left(\exists y_{k-1}\right)\left(\varrho\left(x_{i}, y_{1}\right) \wedge \bigwedge_{i=1}^{k-2} \varrho\left(y_{i}, y_{i+1}\right) \wedge \varrho\left(y_{k-1}, x_{j}\right)\right)
$$

and for an $m$-tuple $\bar{a}$ we express type ${ }_{\varrho}(\bar{a})$ as

$$
\operatorname{type}_{\mathbf{A}}(\bar{a}):=\left\{\varphi_{k}\left(x_{i}, x_{j}\right) \mid 0<k=d\left(a_{i}, a_{j}\right)<\infty\right\} \cup\left\{x_{i}=x_{j} \mid d\left(a_{i}, a_{j}\right)=0\right\}
$$

Again, it is easy to see that type ${ }_{\rho}$ and type ${ }_{\mathrm{A}}$ are equivalent type-functions. Since $0 \leq k \leq p-1$, the maximal possible quantifier depth is $p-2$, so $\mathbf{A}$ is $p-2$-endolocal structure. With a bit more care, we can see that $\mathbf{A}$ is, in fact, 0 -endolocal if $p=2$, and $\frac{p-3}{2}$-endolocal if $p>2$.

2.2.2. The hierarchy of $k$-endolocal structures. The notion of $k$-endolocality defines in a natural way a hierarchy on the class of relational structures. If we denote by $k$-EL $L_{R}$ the class of $k$-endolocal relational structures over the signature $R$, then we have

$$
0-\mathrm{EL}_{R} \subseteq 1-\mathrm{EL}_{R} \subseteq 2-\mathrm{EL}_{R} \subseteq \cdots
$$

Moreover, if we fix a basic set $A$ and if we denote by $k-\mathrm{EL}_{R}(A)$ the class of $k$-endolocal relational structures with the signature $R$ over $A$, then we have

$$
0-\mathrm{EL}_{R}(A) \subseteq 1-\mathrm{EL}_{R}(A) \subseteq 2-\mathrm{EL}_{R}(A) \subseteq \cdots
$$

Whenever we work with such hierarchies, a few natural questions arise: 
1. Are these hierarchies nontrivial?

2. Do they cover all relational structures with the given signature (and the given basic set)?

3. Do they get saturated after finitely many levels?

4. Is it possible to characterize all structures that are placed at the bottom of the hierarchy?

5. Is it possible to determine for the given relational structure whether it is in some class of the hierarchy?

In the sequel we will make attempts to answer some of these questions.

2.2.3. Criteria for $k$-endolocality. A structure $\mathbf{A}$ is $k$-endolocal if for every $m \in$ $\mathbb{N}$, the type-function $\mathrm{pTh}_{\mathrm{A}}^{(k)}$ defines, using the Locality Principle, the closure system $\operatorname{Ext}\left(\mathbb{K}_{m}(\mathbf{A})\right)$. That is, for the given structure $\mathbf{A}$, type-functions $\mathrm{pTh}_{\mathbf{A}}$ and $\mathrm{pTh}_{\mathbf{A}}^{(k)}$ are equivalent. The results that follow characterize $k$-endolocal structures using the language of formal concept analysis.

Definition. We say that a relational structure A is weakly oligomorphic if for all $m \in \mathbb{N}$ the context $\mathbb{K}_{m}(\mathbf{A})$ has just finitely many different extents.

Proposition 1. Let $\mathbf{A}=\left(A,\left(\varrho_{\mathbf{A}}\right)_{\varrho \in R}\right)$ be a relational structure. Then the following hold:

1. If $\mathbf{A}$ is $k$-endolocal, then every intent $\Psi$ of the context $\mathbb{K}_{m}(\mathbf{A}), m \in \mathbb{N}$, is generated by $\Psi^{(k)}$.

2. If $\mathbf{A}$ is weakly oligomorphic and if for all $m \in \mathbb{N}$ every intent $\Psi$ of the context $\mathbb{K}_{m}(\mathbf{A})$ is generated by $\Psi^{(k)}$, then $\mathbf{A}$ is k-endolocal.

Proof. About 1. Let A be $k$-endolocal. Then for every $m \in \mathbb{N}$ and every $\sigma \subseteq A^{m}$ holds 2.2.1(1), Let $\Psi$ be an intent of the context $\mathbb{K}_{m}(\mathbf{A})$ and let $\sigma:=\Psi^{\prime}$. Since $\Psi^{(k)} \subseteq \Psi$, it follows that $\left(\Psi^{(k)}\right)^{\prime} \supseteq \Psi^{\prime}=\sigma$.

On the other hand, from 2.2.1(1) it follows that for every $\bar{a} \in \sigma$ holds $\left\{\bar{b} \in A^{m} \mid\right.$ $\left.\bar{b} \vDash \operatorname{pTh}_{\mathbf{A}}^{(k)}(\bar{a})\right\} \subseteq \sigma$. Note that

$$
\Psi^{(k)}=\left(\sigma^{\prime}\right)^{(k)}=\left(\left(\bigcup_{\bar{a} \in \sigma}\{\bar{a}\}\right)^{\prime}\right)^{(k)}=\left(\bigcap_{\bar{a} \in \sigma}\{\bar{a}\}^{\prime}\right)^{(k)} .
$$

We can now compute $\left(\Psi^{(k)}\right)^{\prime}$ :

$$
\begin{aligned}
\left(\Psi^{(k)}\right)^{\prime} & =\left(\left(\bigcap_{\bar{a} \in \sigma}\{\bar{a}\}^{\prime}\right)^{(k)}\right)^{\prime}=\left(\bigcap_{\bar{a} \in \sigma}\left(\{\bar{a}\}^{\prime}\right)^{(k)}\right)^{\prime}=\sup _{\bar{a} \in \sigma}\left(\left(\{\bar{a}\}^{\prime}\right)^{(k)}\right)^{\prime} \\
& =\sup _{\bar{a} \in \sigma}\left(\operatorname{pTh}_{\mathbf{A}}^{(k)}(\bar{a})\right)^{\prime}=\sup _{\bar{a} \in \sigma}\left\{\bar{b} \in A^{m} \mid \bar{b}=\operatorname{pTh}_{\mathbf{A}}^{(k)}(\bar{a})\right\}
\end{aligned}
$$


and we obtain that $\left(\Psi^{(k)}\right)^{\prime} \subseteq \sigma$. Hence, $\left(\Psi^{(k)}\right)^{\prime \prime}=\sigma^{\prime}=\Psi$.

About 2. Suppose that for every $m \in \mathbb{N}$ and for every intent $\Psi$ of the context $\mathbb{K}_{m}(\mathbf{A})$ holds $\Psi=\left(\Psi^{(k)}\right)^{\prime \prime}$. We will show that 2.2.1(1) holds.

Let $\sigma \subseteq A^{m}$ such that $\sigma=\sigma^{\prime \prime}$. Take $\bar{a} \in \sigma$ and $\bar{b} \in A^{m}$ such that $\bar{b} \vDash \mathrm{pTh}_{\mathbf{A}}^{(k)}(\bar{a})$. Then $\{\bar{b}\}^{\prime} \supseteq\left(\{\bar{a}\}^{\prime}\right)^{(k)}$, so $\bar{b} \in\{\bar{b}\}^{\prime \prime} \subseteq\left(\left(\{\bar{a}\}^{\prime}\right)^{(k)}\right)^{\prime}=\{\bar{a}\}^{\prime \prime} \subseteq \sigma$.

Let now $\sigma \subseteq A^{m}$ and suppose that for all $\bar{a} \in \sigma$ and $\bar{b} \in A^{m}$ holds that if $\bar{b} \vDash \operatorname{pTh}_{\mathbf{A}}^{(k)}(\bar{a})$, then $\bar{b} \in \sigma$. Note that $\sigma \subseteq \sigma^{\prime \prime}$ always holds, so we only have to show the other inclusion. Let $\Psi:=\sigma^{\prime}$. Then

$$
\begin{aligned}
\sigma^{\prime \prime} & =\Psi^{\prime}=\left(\Psi^{(k)}\right)^{\prime \prime \prime}=\left(\Psi^{(k)}\right)^{\prime}=\left\{\bar{b} \in A^{m} \mid \bar{b} \models \Psi^{(k)}\right\} \\
& =\left\{\bar{b} \in A^{m} \mid \bar{b} \models \operatorname{pTh}_{\mathbf{A}}^{(k)}(\sigma)\right\}=\left\{\bar{b} \in A^{m} \mid \bar{b} \models \bigcap_{\bar{a} \in \sigma} \operatorname{pTh}_{\mathbf{A}}^{(k)}(\bar{a})\right\} .
\end{aligned}
$$

Hence,

$$
\bar{b} \in \sigma^{\prime \prime} \Leftrightarrow \bar{b} \vDash \bigcap_{\bar{a} \in \sigma} \operatorname{pTh}_{\mathbf{A}}^{(k)}(\bar{a}) \Leftrightarrow \bar{b} \in\left(\bigcap_{\bar{a} \in \sigma} \operatorname{pTh}_{\mathbf{A}}^{(k)}(\bar{a})\right)^{\prime} \Leftrightarrow \bar{b} \in \sup _{\bar{a} \in \sigma}\left(\operatorname{pTh}_{\mathbf{A}}^{(k)}(\bar{a})\right)^{\prime}
$$

Since $\mathbf{A}$ is weakly oligomorphic, there are elements $\bar{a}_{1}, \ldots, \bar{a}_{l} \in \sigma$ such that

$$
\sup _{\bar{a} \in \sigma}\left(\operatorname{pTh}_{\mathbf{A}}^{(k)}(\bar{a})\right)^{\prime}=\bigcup_{i=1}^{l}\left(\operatorname{pTh}_{\mathbf{A}}^{(k)}\left(\bar{a}_{i}\right)\right)^{\prime} \subseteq \sigma .
$$

Thus, $\sigma^{\prime \prime} \subseteq \sigma$, so $\sigma=\sigma^{\prime \prime}$, which ends this proof.

The previous Proposition allows us to decide $k$-endolocality of a given relational structure $\mathbf{A}$ by studying those intents of $\mathbb{K}_{m}(\mathbf{A})$ that are generated by their fragments of quantifier depth at most $k$. This motivates us to introduce contexts $\mathbb{K}_{m}^{(k)}(\mathbf{A}):=\left(A^{m}, \Phi_{m}^{(k)}(\exists, \wedge, \vee,=, R), \models\right)$, for $k \in \mathbb{N}$, and to study their concept lattices. In order not to cause confusions, the Galois-operators of $\mathbb{K}_{m}^{(k)}(\mathbf{A})$ will be denoted by ${ }^{+}$instead of '.

Lemma. Let $\mathbf{A}=\left(A,\left(\varrho_{\mathbf{A}}\right)_{\varrho \in R}\right)$ be a relational structure. Then the following are equivalent:

1. Every intent $\Psi$ in $\mathbb{K}_{m}(\mathbf{A})$ is generated by $\Psi^{(k)}$.

2. $\operatorname{Ext}\left(\mathbb{K}_{m}(\mathbf{A})\right)=\operatorname{Ext}\left(\mathbb{K}_{m}^{(k)}(\mathbf{A})\right)$.

Proof. $(\mathbf{1} \Rightarrow \mathbf{2})$ Suppose that every intent $\Psi$ in $\mathbb{K}_{m}(\mathbf{A})$ is generated by $\Psi^{(k)}$, i.e. $\Psi=\left(\Psi^{(k)}\right)^{\prime \prime}$. Every extent in $\mathbb{K}_{m}(\mathbf{A})$ is then of the form

$$
\Psi^{\prime}=\left(\Psi^{(k)}\right)^{\prime \prime \prime}=\left(\Psi^{(k)}\right)^{\prime}=\left(\Psi^{(k)}\right)^{+}
$$

Since $\left(\Psi^{(k)}\right)^{+} \in \operatorname{Ext}\left(\mathbb{K}_{m}^{(k)}(\mathbf{A})\right)$, it follows that $\operatorname{Ext}\left(\mathbb{K}_{m}(\mathbf{A})\right) \subseteq \operatorname{Ext}\left(\mathbb{K}_{m}^{(k)}(\mathbf{A})\right)$. On the other hand, for $\sigma \in \operatorname{Ext}\left(\mathbb{K}_{m}^{(k)}(\mathbf{A})\right)$, there exists a $\Psi \subseteq \Phi_{m}^{(k)}(\exists, \wedge, \vee,=, R)$ such that 
$\sigma=\Psi^{+}$. Since $\Phi_{m}^{(k)}(\exists, \wedge, \vee,=, R) \subseteq \Phi_{m}(\exists, \wedge, \vee,=, R)$, it follows that $\Psi^{+}=\Psi^{\prime}$, so $\operatorname{Ext}\left(\mathbb{K}_{m}^{(k)}(\mathbf{A})\right) \subseteq \operatorname{Ext}\left(\mathbb{K}_{m}(\mathbf{A})\right)$.

$(\mathbf{2} \Rightarrow \mathbf{1})$ Let $\Psi$ be an arbitrary intent in $\mathbb{K}_{m}(\mathbf{A})$. Then $\Psi^{\prime} \in \operatorname{Ext}\left(\mathbb{K}_{m}(\mathbf{A})\right)$, so $\Psi^{\prime} \in$ $\operatorname{Ext}\left(\mathbb{K}_{m}^{(k)}(\mathbf{A})\right)$, i.e. $\left(\Psi^{\prime}\right)^{++}=\Psi^{\prime}$. We have to show that $\Psi=\left(\Psi^{(k)}\right)^{\prime \prime}$. Since $\left(\Psi^{(k)}\right) \subseteq \Psi$, it follows that $\Psi^{\prime} \subseteq\left(\Psi^{(k)}\right)^{\prime}=\left(\Psi^{(k)}\right)^{+}$, so we obtain $\Psi^{(k)} \subseteq\left(\Psi^{(k)}\right)^{++} \subseteq \Psi^{\prime+}$. On the other hand, $\Psi^{\prime+} \subseteq \Psi^{(k)}$, so $\Psi^{\prime+}=\Psi^{(k)}$. From this fact we obtain

$$
\left(\Psi^{(k)}\right)^{\prime \prime}=\left(\left(\Psi^{(k)}\right)^{\prime}\right)^{\prime}=\left(\left(\Psi^{(k)}\right)^{+}\right)^{\prime}=\left(\left(\Psi^{\prime+}\right)^{+}\right)^{\prime}=\left(\left(\Psi^{\prime}\right)^{++}\right)^{\prime}=\left(\left(\Psi^{\prime}\right)^{\prime}=\Psi .\right.
$$

Corollary. Let $\mathbf{A}=\left(A,\left(\varrho_{\mathbf{A}}\right)_{\varrho \in R}\right)$ be a relational structure. Then the following hold:

1. If $\mathbf{A}$ is k-endolocal, then $\operatorname{Ext}\left(\mathbb{K}_{m}(\mathbf{A})\right)=\operatorname{Ext}\left(\mathbb{K}_{m}^{(k)}(\mathbf{A})\right)$.

2. If $\mathbf{A}$ is weakly oligomorphic and $\operatorname{Ext}\left(\mathbb{K}_{m}(\mathbf{A})\right)=\operatorname{Ext}\left(\mathbb{K}_{m}^{(k)}(\mathbf{A})\right)$, then $\mathbf{A}$ is $k$-endolocal. Remark. An immediate consequence of the previous corollary is that $\mathbb{K}_{m}(\mathbf{A})$ and $\mathbb{K}_{m}^{(k)}(\mathbf{A})$ have isomorphic closure systems of intents. The isomorphism between these closure systems maps every intent $\Psi$ of $\mathbb{K}_{m}(\mathbf{A})$ to $\Psi^{(k)}$.

Proposition 2. Let $\mathbf{A}=\left(A,\left(\varrho_{\mathbf{A}}\right)_{\varrho \in R}\right)$ be a relational structure. Then the following hold:

1. If $\mathbf{A}$ is $k$-endolocal, then for

$$
\forall \bar{a}, \bar{b} \in A^{m}: \quad \operatorname{pTh}_{\mathbf{A}}^{(k)}(\bar{a}) \subseteq \operatorname{pTh}_{\mathbf{A}}^{(k)}(\bar{b}) \Leftrightarrow \operatorname{pTh}_{\mathbf{A}}(\bar{a}) \subseteq \operatorname{pTh}_{\mathbf{A}}(\bar{b}) .
$$

2. If $\mathbf{A}$ is weakly oligomorphic and (1) holds, then $\mathbf{A}$ is k-endolocal.

Proof. About 1. Let A be k-endolocal. Then (1) follows immediately from the previous Remark.

About 2, We will show that $\mathbb{K}_{m}(\mathbf{A})$ and $\mathbb{K}_{m}^{(k)}(\mathbf{A})$ have the same extents.

Let $\sigma$ be an extent of $\mathbb{K}_{m}^{(k)}(\mathbf{A})$. Then $\sigma^{+} \subseteq \sigma^{\prime}$, so $\sigma=\sigma^{\prime \prime} \supseteq \sigma^{++}$. Since $\sigma \subseteq \sigma^{\prime \prime}$, it follows $\sigma=\sigma^{\prime \prime}$, so $\sigma \in \operatorname{Ext}\left(\mathbb{K}_{m}(\mathbf{A})\right)$.

Let, now, $\sigma$ be an extent of $\mathbb{K}_{m}(\mathbf{A})$. Note that Condition (1) is equivalent to

$$
\forall \bar{a}, \bar{b} \in A^{m}: \quad \bar{b} \in\left(\operatorname{pTh}_{\mathbf{A}}^{(k)}(\bar{a})\right)^{\prime} \Leftrightarrow \bar{b} \in\left(\operatorname{pTh}_{\mathbf{A}}(\bar{a})\right)^{\prime},
$$

i.e. for every $\bar{a} \in A^{m}$ we have that $\{\bar{a}\}^{\prime \prime}=\{\bar{a}\}^{++}$. Since $\mathbf{A}$ is weakly olgomorphic, $\mathbb{K}_{m}(\mathrm{~A})$ has only finitely many pairwise different extents $\{\bar{a}\}^{\prime \prime}$. Moreover, for every extent $\sigma$ of $\mathbb{K}_{m}(\mathbf{A})$ we know that

$$
\sigma=\bigcup_{\bar{a} \in \sigma}\{\bar{a}\}^{\prime \prime},
$$

so we can choose representatives $\bar{a}_{1}, \ldots, \bar{a}_{l} \in \sigma$ such that

$$
\sigma=\bigcup_{i=1}^{l}\left\{\bar{a}_{i}\right\}^{\prime \prime}=\bigcup_{i=1}^{l}\left\{\bar{a}_{i}\right\}^{++} \in \operatorname{Ext}\left(\mathbb{K}_{m}^{(k)}(\mathbf{A})\right) .
$$

Hence, since $\mathbf{A}$ is weakly oligomorphic, from the above Corollary it follows that $\mathbf{A}$ is k-endolocal. 
2.2.4. Structures that are not $k$-endolocal. The definition of $k$-endolocality is useful if we want to prove that a given structure has this property. On the other hand, if we want to identify endolocal structures in a given set of relational structures, it is more useful to filter out those that are not $k$-endolocal. The following proposition is the first step in our attempts to find satisfactory criteria for recognizing structures that are not $k$-endolocal.

Proposition. Let $\mathbf{A}=\left(A,\left(\varrho_{\mathbf{A}}\right)_{\varrho \in R}\right)$ be a weakly oligomorphic relational structure. Then the following are equivalent:

1. There exists an $m \in \mathbb{N}$, an extent $\sigma$ of $\mathbb{K}_{m}(\mathbf{A})$, an $\bar{a} \in \sigma$ and some $\bar{b} \in A^{m}$ such that $\operatorname{pTh}_{\mathbf{A}}^{(k)}(\bar{a}) \subseteq \operatorname{pTh}_{\mathbf{A}}^{(k)}(\bar{b})$, but $\bar{b} \notin \sigma$.

2. There exists an $m \in \mathbb{N}$, and $\bar{a}, \bar{b} \in A^{m}$ such that $\mathrm{pTh}_{\mathbf{A}}^{(k)}(\bar{a}) \subseteq \operatorname{pTh}_{\mathbf{A}}^{(k)}(\bar{b})$, but $\operatorname{pTh}_{\mathbf{A}}(\bar{a}) \nsubseteq \operatorname{pTh}_{\mathbf{A}}(\bar{b})$.

3. A is not k-endolocal.

Proof. $(\mathbf{1} \Leftrightarrow \mathbf{2})$ Let 1 hold and suppose that for $\bar{a}$ and $\bar{b}$ from 1 holds that

$$
\operatorname{pTh}_{\mathbf{A}}(\bar{a}) \subseteq \operatorname{pTh}_{\mathbf{A}}(\bar{b}) .
$$

Then $\bar{b} \vDash \mathrm{pTh}_{\mathrm{A}}(\bar{a})=\{\bar{a}\}^{\prime}$, so $\bar{b} \in\{\bar{a}\}^{\prime \prime}$. On the other hand, $\{\bar{a}\} \subseteq \sigma$, so $\{\bar{a}\}^{\prime \prime} \subseteq \sigma^{\prime \prime}=\sigma$, and, therefore, $\bar{b} \in \sigma$, which is a contradiction.

Hence, $\operatorname{pTh}_{\mathrm{A}}(\bar{a}) \nsubseteq \mathrm{pTh}_{\mathrm{A}}(\bar{b})$.

Let now 2 hold, and take $\bar{a}$ and $\bar{b}$ such that $\mathrm{pTh}_{\mathrm{A}}^{(k)}(\bar{a}) \subseteq \mathrm{pTh}_{\mathbf{A}}^{(k)}(\bar{b})$, but $\mathrm{pTh}_{\mathbf{A}}(\bar{a}) \nsubseteq$ $\operatorname{pTh}_{\mathbf{A}}(\bar{b})$. We define $\sigma:=\{\bar{a}\}^{\prime \prime}$. Then $\sigma=\sigma^{\prime \prime}, \bar{a} \in \sigma$, but $\bar{b} \notin \sigma$.

$(2 \Leftrightarrow 3)$ This is a direct consequence of 2.2.3(Proposition 2),

Remark. Note that $(\mathbf{1} \Leftrightarrow \mathbf{2})$ and $(\mathbf{2} \Rightarrow \mathbf{3})$ also hold in general, i.e. they do not depend on whether $\mathbf{A}$ is weakly oligomorphic or not.

2.2.5. $k$-endolocality and finite structures. We turn now our attention again to finite relational structures. The following Proposition gives answers to Questions 2 and 3 from 2.2.2. In particular, we will show that every finite relational structure is $k$-endolocal for a certain $k \in \mathbb{N}$, where $k$ is bounded by the cardinality of the basic set.

Proposition. Let $\mathbf{A}$ be a relational structure and let $m \in \mathbb{N}$. Then for every $\bar{b} \in A^{m}$ holds $\mathrm{pTh}_{\mathbf{A}}(\bar{b})=\left(\mathrm{pTh}_{\mathbf{A}}^{(|A|)}(\bar{b})\right)^{\prime \prime}$

Proof. Note that from $\operatorname{pTh}_{\mathbf{A}}(\bar{b}) \supseteq \operatorname{pTh}_{\mathbf{A}}^{(|A|)}(\bar{b})$ it follows $\operatorname{pTh}_{\mathbf{A}}(\bar{b})=\left(\operatorname{pTh}_{\mathbf{A}}(\bar{b})\right)^{\prime \prime} \supseteq$ $\left(\mathrm{pTh}_{\mathrm{A}}^{(|A|)}(\bar{b})\right)^{\prime \prime}$. We will prove that the other inclusion also holds.

Let $\varphi \in \mathrm{pTh}_{\mathbf{A}}(\bar{b})$. Then

$$
\varphi \equiv \bigvee_{i \in I}\left(\exists x_{m+1}\right) \ldots\left(\exists x_{m+l}\right) \bigwedge_{j \in J_{i}} \psi_{i j}
$$


Denote by $\psi_{i}$ the formula $\left(\exists x_{m+1}\right) \ldots\left(\exists x_{m+l}\right) \bigwedge_{j \in J_{i}} \psi_{i j}$. For each $\psi_{i}$ and for each $\bar{a} \in A^{m}$ such that $\bar{a} \vDash \psi_{i}$, we will now construct a formula $\psi_{i, \bar{a}}$ with the following three properties:

1. $\psi_{i, \bar{a}}^{\mathbf{A}} \subseteq \psi_{i}^{\mathbf{A}}$,

2. $\bar{a} \vDash \psi_{i, \bar{a}}$ and

3. $\operatorname{qd}\left(\psi_{i, \bar{a}}\right) \leq|A|$.

By 0.2.3(Proposition), there exists a relational configuration $(\mathbf{C}, \mathbf{D}, E, \theta)$ such that just for finitely many $\varrho \in R$ we have that $\varrho_{\mathbf{D}} \neq \emptyset$ and such that for all $\bar{a} \in A^{m}$ holds

$$
\bar{a} \vDash \psi_{i} \Leftrightarrow \bar{a} \|(\mathbf{C}, \mathbf{D}, E, \theta) .
$$

In other words, there exists a homomorphism $g: \mathbf{D} \rightarrow \mathbf{A}$ such that (G1) and (G2) hold.

We define an equivalence relation $\simeq$ over $D$ in the following way:

$$
d \simeq e \text { if and only if } d=e \text { or }(d, e \in D \backslash C \text { and }(d, e) \in \operatorname{ker} g) .
$$

Note that this equivalence relation has at most $|A|+|C|$ equivalence classes.

We construct $(\tilde{\mathbf{C}}, \tilde{\mathbf{D}}, \tilde{E}, \tilde{\theta})$ such that $\tilde{D}:=D / \simeq, \tilde{C}:=\{\{c\} \mid c \in C\}, \tilde{E}: i \mapsto\{E(i)\}$, $\tilde{\theta}:=\Delta_{\tilde{D}} \cup\left\{\left(\left[c_{j_{1}}\right]_{\simeq},\left[c_{j_{2}}\right]_{\simeq}\right) \mid\left(c_{j_{1}}, c_{j_{2}}\right) \in \theta\right\}$ and define $\tilde{g}: \tilde{\mathbf{D}} \rightarrow \mathbf{A}:[c]_{\simeq} \mapsto g(c)$.

If $[d]_{\simeq}=[e]_{\simeq}$, then $d \simeq e$, so $(d, e) \in$ ker $g$, i.e. $g(d)=g(e)$, and, therefore, $\tilde{g}$ is well defined. Further, let us define for every $\varrho \in R, \varrho_{\tilde{\mathbf{D}}}:=\left\{\left(\left[d_{i_{1}}\right]_{\simeq}, \ldots,\left[d_{i_{\operatorname{ar}(\varrho)}}\right]_{\simeq}\right) \mid\right.$ $\left.\left(d_{i_{1}}, \ldots, d_{i_{\operatorname{ar}(\varrho)}}\right) \in \varrho_{\mathbf{D}}\right\}$. Let $\left(e_{1}, \ldots, e_{\operatorname{ar}(\varrho)}\right) \in \varrho_{\tilde{\mathbf{D}}}$. Then there exists a $\left(d_{i_{1}}, \ldots, d_{i_{\text {ar }(\varrho)}}\right) \in$ $\varrho_{\text {D }}$ such that $e_{j}=\left[d_{i_{j}}\right]_{\simeq}, j=1, \ldots, \operatorname{ar}(\varrho)$. By definition, $\left(\tilde{g}\left(e_{1}\right), \ldots, \tilde{g}\left(e_{\operatorname{ar}(\varrho)}\right)\right)=$ $\left(g\left(d_{i_{1}}\right), \ldots, g\left(d_{i_{\text {ar(o) }}}\right)\right) \in \varrho_{\mathbf{A}}$, so $\tilde{g}$ is a homomorphism. From the definition of $\tilde{C}$ and $\tilde{E}$, it follows that (G1) holds, while (G2) follows from the definition of $\tilde{\theta}$.

Observe that from this it follows that $\bar{a} \|(\tilde{\mathbf{C}}, \tilde{\mathbf{D}}, \tilde{E}, \tilde{\theta})$.

We will now show that if $\bar{t} \|(\tilde{\mathbf{C}}, \tilde{\mathbf{D}}, \tilde{E}, \tilde{\theta})$, then $\bar{t} \|(\mathbf{C}, \mathbf{D}, E, \theta)$.

Let $\bar{t} \Vdash(\tilde{\mathbf{C}}, \tilde{\mathbf{D}}, \tilde{E}, \tilde{\theta})$. Then there exists a homomorphism $\tilde{g}_{t}: \tilde{\mathbf{D}} \rightarrow$ A that fulfills (G1) and (G2). We construct $g_{t}: \mathbf{D} \rightarrow \mathbf{A}$ as $g_{t}:=\tilde{g}_{t} \circ$ nat $\simeq$. Then $g_{t}$ is both well-defined and a homomorphism. For (G1) note that $g_{t}(E(i))=\tilde{g}_{t}\left(\right.$ nat $\left._{\simeq}(E(i))\right)=\tilde{g}_{t}\left([E(i)]_{\simeq}\right)=a_{i}$, since $\tilde{g}_{t}$ fulfills (G1), For (G2) take $\left(c_{j_{1}}, c_{j_{2}}\right) \in \theta$. Then $\left(\left[c_{j_{1}}\right]_{\simeq},\left[c_{j_{2}}\right]_{\simeq}\right) \in \tilde{\theta} \subseteq$

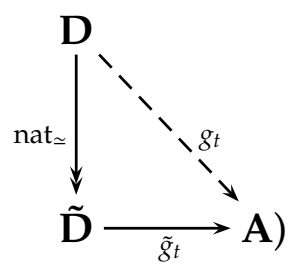
$\operatorname{ker} \tilde{g}_{t}$, so $g_{t}\left(c_{j_{1}}\right)=\tilde{g}_{t}\left(\left[c_{j_{1}}\right]_{\simeq}\right)=\tilde{g}_{t}\left(\left[c_{j_{2}}\right]_{\simeq}\right)=g_{t}\left(c_{j_{2}}\right)$, and $\theta \subseteq \operatorname{ker} g_{t}$.

Note that $\varrho_{\tilde{\mathbf{D}}} \neq \emptyset$ if and only if $\varrho_{\mathbf{D}} \neq \emptyset$. Thus, there are just finitely many $\varrho \in R$ with $\varrho_{\tilde{\mathbf{D}}} \neq \emptyset$. Hence, by 0.2 (Proposition), it follows that there exists a formula $\psi_{i, \bar{a}}$ such that $\operatorname{qd}\left(\psi_{i, \bar{a}}\right)=|\tilde{D} \backslash \hat{C}| \leq|A|$ and

$$
\bar{t} \models \psi_{i, \bar{a}} \Leftrightarrow \bar{t} \Vdash(\tilde{\mathbf{C}}, \tilde{\mathbf{D}}, \tilde{E}, \tilde{\theta}) .
$$


We take now $\bigvee_{\left\{\bar{a} \mid \bar{a} \models \psi_{i}\right\}} \psi_{i, \bar{a}}$ and we claim that it is equivalent to $\psi_{i}$. If $\bar{t} \vDash \psi_{i}$, then $\bar{t} \models \psi_{i, \bar{t}}$, so $\bar{t} \models \bigvee_{\left\{\bar{a} \mid \bar{a} \models \psi_{i}\right\}} \psi_{i, \bar{a}}$. On the other hand, if $\bar{t} \vDash \bigvee_{\left\{\bar{a} \mid \bar{a} \models \psi_{i}\right\}} \psi_{i, \bar{a}}$, then there exists an $\bar{a}$ such that $\bar{a} \vDash \psi_{i}$ and $\bar{t} \vDash \psi_{i, \bar{a}}$, so $\bar{t} \vDash \psi_{i}$.

Since $\operatorname{qd}\left(\psi_{i, \bar{a}}\right) \leq|A|$, it follows that $\operatorname{qd}\left(\bigvee_{\left\{\bar{a} \mid \bar{a} \models \psi_{i}\right\}} \psi_{i, \bar{a}}\right) \leq|A|$, so $\operatorname{qd}\left(\bigvee_{i \in I} \psi_{i}\right) \leq|A|$, i.e. $\varphi$ is equivalent to a formula of quantifier depth less than or equal $|A|$, and, hence, $\varphi \in\left(\mathrm{pTh}_{\mathrm{A}}^{|A|}(\bar{b})\right)^{\prime \prime}$.

\subsection{Model-theoretical characterizations of endolocality}

2.3.1. Positive existential elimination sets. In the previous section we were using the language of formal concept analysis to define $k$-endolocal relational structures and to describe some of their basic properties. Here we will create a connection between the notion of $k$-endolocality and notions from model theory. Let us start by introducing the notion of positive existential elimination sets.

Definition. Let $\mathbf{A}=\left(A,\left(\varrho_{\mathbf{A}}\right)_{\varrho \in R}\right)$ be a relational structure. A set $\Psi \subseteq \Phi(\exists, \wedge, \vee,=$ $, R)$ is called a positive existential elimination set for $\mathbf{A}$ if for every formula $\varphi\left(x_{1}, \ldots, x_{m}\right) \in \Phi(\exists, \wedge, \vee,=, R)$ there is a formula $\varphi^{*}\left(x_{1}, \ldots, x_{m}\right) \in \Phi(\exists, \wedge, \vee,=, R)$ which is a disjunction of conjunctions of formulae in $\Psi$ and $\varphi$ is equivalent to $\varphi^{*}$ in $\mathbf{A}$.

Proposition 1. Let $\mathbf{A}=\left(A,\left(\varrho_{\mathbf{A}}\right)_{\varrho \in R}\right)$ be a weakly oligomorphic relational structure. If $\Phi^{(k)}(\exists, \wedge, \vee,=, R)$ is a positive existential elimination set for $\mathbf{A}$ then $\mathbf{A}$ is $k$-endolocal.

Proof. Suppose that $\Phi^{(k)}(\exists, \wedge, \vee,=, R)$ is a positive existential elimination set for A. Since A is weakly oligomorphic, by 2.2.3(Proposition 1), it suffices to show that for every $m \in \mathbb{N}$, we have that every intent $\Psi$ of the context $\mathbb{K}_{m}(\mathbf{A})$ is generated by $\Psi^{(k)}$.

Take any intent $\Psi$ of $\mathbb{K}_{m}(\mathbf{A})$. For each $\psi \in \Psi$, let $\psi^{*}$ be a disjunction of conjunctions of formulae from $\Phi^{(k)}(\exists, \wedge, \vee,=, R)$ that is equivalent to $\psi$ in A. Note that $\psi^{*} \in \Psi$. It is not hard to see that

$$
\psi^{*} \equiv \bigwedge_{i \in I_{\psi}} \psi_{i}^{*}
$$

where $\psi_{i}^{*}, i \in I_{\psi}$, are disjunctions of formulae from $\Phi^{(k)}(\exists, \wedge, \vee,=, R)$. In particular, for every $i \in I_{\psi}$ we have that $\psi_{i}^{*} \in \Phi^{(k)}(\exists, \wedge, \vee,=, R)$. Further, let $\Psi^{*}:=\left\{\psi_{i}^{*} \mid \psi \in\right.$ $\left.\Psi, \quad i \in I_{\psi}\right\}$. Then

$$
\Psi^{\prime}=\bigcap_{\psi \in \Psi} \psi^{\prime}=\bigcap_{\psi \in \Psi}\left(\psi^{*}\right)^{\prime}=\bigcap_{\psi \in \Psi} \bigcap_{i \in I_{\psi}}\left(\psi_{i}^{*}\right)^{\prime}=\left(\Psi^{*}\right)^{\prime},
$$

so $\Psi=\Psi^{\prime \prime}=\left(\Psi^{*}\right)^{\prime \prime}$. Now we note that from $\psi^{*} \in \Psi$, it follows that

$$
\Psi^{\prime} \subseteq\left\{\psi^{*}\right\}^{\prime}=\left\{\bigwedge_{i \in I_{\psi}} \psi_{i}^{*}\right\}^{\prime}=\left\{\psi_{i}^{*} \mid i \in I_{\psi}\right\}^{\prime} .
$$


Since $\Psi$ is an intent, it follows that $\left\{\psi_{i}^{*} \mid i \in I_{\psi}\right\} \subseteq \Psi^{(k)}$. Hence, $\Psi^{*} \subseteq \Psi^{(k)}$, and, thus, $\left(\Psi^{(k)}\right)^{\prime \prime}=\Psi$.

Remark. In the previous proof we have shown that if a relational structure $\mathbf{A}$ has $\Phi^{(k)}(\exists, \wedge, \vee,=, R)$ as a positive existential elimination set, then every intent $\Psi$ of $\mathbb{K}_{m}(\mathrm{~A}), m \in \mathbb{N}$ is generated by $\Psi^{(k)}$. From 2.2.3(Lemma) it follows that this is equivalent to the fact that $\operatorname{Ext}\left(\mathbb{K}_{m}(\mathbf{A})\right)=\operatorname{Ext}\left(\mathbb{K}_{m}^{(k)}(\mathbf{A})\right)$.

Proposition 2. Let $\mathbf{A}=\left(A,\left(\varrho_{\mathbf{A}}\right)_{\varrho \in R}\right)$ be a weakly oligomorphic relational structure. If A is k-endolocal then $\Phi^{(k)}(\exists, \wedge, \vee,=, R)$ is a positive existential elimination set for $\mathbf{A}$.

Proof. Let $\mathbf{A}$ be a $k$-endolocal weakly oligomorphic relational structure. Further, let $m \in \mathbb{N}$ and let $\psi \in \Phi_{m}(\exists, \wedge, \vee,=, R)$. Then, by 2.2.3(Proposition 1) and by 2.2.3(Lemma), we have that $\operatorname{Ext}\left(\mathbb{K}_{m}(\mathbf{A})\right)=\operatorname{Ext}\left(\mathbb{K}_{m}^{(k)}(\mathbf{A})\right)$, so $\{\psi\}^{\prime}=\left(\left(\{\psi\}^{\prime \prime}\right)^{(k)}\right)^{\prime}$. Since $\mathbf{A}$ is weakly oligomorphic, the context $\mathbb{K}_{m}(\mathbf{A})$ has finitely many extents. From this it follows at once that the cross-table of $\mathbb{K}_{m}^{(k)}(\mathbf{A})$ has just finitely many distinct columns. So, there exist $\varphi_{1}, \ldots, \varphi_{l} \in\left(\{\psi\}^{\prime \prime}\right)^{(k)}$ such that

$$
\{\psi\}^{\prime}=\left(\left(\{\psi\}^{\prime \prime}\right)^{(k)}\right)^{\prime}=\left\{\varphi_{1}, \ldots, \varphi_{l}\right\}^{\prime}=\left\{\varphi_{1} \wedge \cdots \wedge \varphi_{l}\right\}^{\prime}
$$

Hence, $\psi$ is equivalent to $\varphi_{1} \wedge \cdots \wedge \varphi_{l}$ in $\mathbf{A}$, that is to a conjunction of formulae from $\Phi_{m}^{(k)}(\exists, \wedge, \vee,=, R)$.

2.3.2. One-point extension and weak one-point extension. In contrast to the previous sections, where the general notion of $k$-endolocality was handled, here we direct our attention to endolocal relational structures. The second notion from model theory that is is closely connected to the notion of endolocality is the weak homogeneity of relational structures. Let us recall the original definition of a weakly homogeneous relational structure:

Definition 1. We say that a relational structure $\mathbf{D}$ is weakly homogeneous if for every two finite substructures $\mathbf{A}$ and $\mathbf{B}$ of $\mathbf{D}$ such that $\mathbf{A} \leq \mathbf{B}$, and for every embedding $f: \mathbf{A} \hookrightarrow \mathbf{D}$ there exists an embedding $g: \mathbf{B} \hookrightarrow \mathbf{D}$ that extends $f$.

In this study, we are not focused on embeddings, but on local homomorphisms. Therefore, we introduce the notion of weakly homomorphism-homogeneous structures:

Definition 2. We say that a relational structure D is weakly homomorphismhomogeneous if for every two finite substructures $\mathbf{A}$ an $\mathbf{B}$ of $\mathbf{D}$ such that $\mathbf{A} \leq \mathbf{B}$, and for every local homomorphism $f: \mathbf{A} \rightarrow \mathbf{D}$ there exists a local homomorphism $g: \mathbf{B} \rightarrow \mathbf{D}$ that extends $f$.

Let us now formulate another property that is equivalent to weak homomorphism-homogeneity and that will be useful when we characterize structures that are not endolocal: 
Definition 3. We say that a relational structure A has the one-point extension property if for every finite substructure $\mathbf{B}$ of $\mathbf{A}$, every $b \in A \backslash B$ and every homomorphism $f: \mathbf{B} \rightarrow \mathbf{A}$, there exists a homomorphism $g: \mathbf{B} \cup\{b\} \rightarrow \mathbf{A}$ which extends $f$.

The following Lemma will prove useful later on.

Lemma 1. Let $\mathbf{A}=\left(A,\left(\varrho_{\mathbf{A}}\right)_{\varrho \in R}\right)$ be a relational structure and let $\mathbf{B}=\left(B,\left(\varrho_{\mathbf{B}}\right)_{\varrho \in R}\right)$ be a retract of $\mathbf{A}$. If $\mathbf{A}$ has the one-point extension property, then $\mathbf{B}$ also has the one-point extension property.

Proof. Let $r: \mathbf{A} \rightarrow \mathbf{B}$ be a retraction. Without loss of generality, we can assume that $\mathbf{B} \leq \mathbf{A}$. Let $\iota: \mathbf{B} \hookrightarrow \mathbf{A}$ be the identical embedding. Let $B_{1} \subseteq B$ and let $f: \mathbf{B}_{1} \rightarrow \mathbf{B}$ be a local homomorphism. We will take a $b \in B \backslash B_{1}$ and we will show that $f$ can be extended to $\mathbf{B}_{1} \cup\{b\}$. Note that $\iota \circ f: \mathbf{B}_{1} \rightarrow \mathbf{A}$ is also a local homomorphism, and, since $\mathbf{A}$ has the one-point extension property, it can be extended to $\tilde{f}: \mathbf{B}_{\mathbf{1}} \cup\{b\} \rightarrow \mathbf{A}$. We claim that $g=r \circ \tilde{f}$ is an extension of $f$ to $\mathbf{B}_{1} \cup\{b\}$. Take $x \in B_{1}$. Then

$$
g(x)=(r \circ \tilde{f})(x)=r(\tilde{f}(x))=r((\iota \circ f)(x))=((r \circ \iota) \circ f)(x)=f(x) .
$$

Hence, $\mathbf{B}$ has the one-point extension property.

Lemma 2. Let $\mathbf{A}=\left(A,\left(\varrho_{\mathbf{A}}\right)_{\varrho \in R}\right)$ be a weakly oligomorphic relational structure. If $\mathbf{A}$ does not have the one-point-extension property, then there exist an $m \in \mathbb{N}$, an irreflexive tuple $\bar{a} \in A^{m}$, some $\bar{b} \in A^{m}$ and a formula $\varphi \equiv\left(\exists x_{m+1}\right) \psi$, where $\psi$ is a conjunction of atomic formulae from $\Phi_{m+1}^{(0)}(\exists, \wedge, \vee,=, R)$, such that $\mathrm{pTh}_{\mathrm{A}}^{(0)}(\bar{a}) \subseteq \mathrm{pTh}_{\mathrm{A}}^{(0)}(\bar{b})$, and $\bar{a} \vDash \varphi$, but $\bar{b} \not=\varphi$.

Proof. Suppose that A does not have the one-point extension property. This means that there exist a finite substructure $\mathbf{B}$ of $\mathbf{A}$, a homomorphism $f: \mathbf{B} \rightarrow \mathbf{A}$, and an element $d \in A \backslash B$, such that $f$ cannot be extended to a homomorphism between $\mathbf{B} \cup\{d\}$ and $\mathbf{A}$.

Let $m:=|B|, B \cup\{d\}:=\left\{b_{1}, \ldots, b_{m+1}\right\}$ such that $B=\left\{b_{1}, \ldots, b_{m}\right\}$ and $d=b_{m+1}$. Define $\bar{a}:=\left(b_{1}, \ldots, b_{m}\right)$ and $\bar{b}=\left(f\left(b_{1}\right), \ldots, f\left(b_{m}\right)\right)$. Consider the following set of atoms

$$
\Phi:=\left\{\varrho\left(x_{i_{1}}, \ldots, x_{i_{\operatorname{ar}(\varrho)}}\right) \mid \varrho \in R, \quad i_{1}, \ldots, i_{\operatorname{ar}(\varrho)} \in\{1, \ldots, m+1\},\left(b_{i_{1}}, \ldots, b_{i_{\text {ar }(\varrho)}}\right) \in \varrho_{\mathbf{A}}\right\} .
$$

Clearly, $\Phi \subseteq \Phi_{m+1}^{(0)}(\exists, \wedge, \vee,=, R)$, but it might be infinite. However, since $\mathbf{A}$ is weakly oligomorphic, $\Phi$ falls into finitely many equivalence classes with respect to equivalence of formulae in A. Thus, we can choose formulae $\psi_{1}, \ldots, \psi_{l} \in \Phi$ such that

$$
\Phi^{\prime}=\left\{\psi_{1}, \ldots, \psi_{l}\right\}^{\prime}=\left\{\psi_{1} \wedge \cdots \wedge \psi_{l}\right\}^{\prime} .
$$

We define $\psi \equiv \psi_{1} \wedge \cdots \wedge \psi_{l}$, and $\varphi \equiv\left(\exists x_{m+1}\right) \psi$. 
It is easy to see that $\mathrm{pTh}_{\mathbf{A}}^{(0)}(\bar{a}) \subseteq \mathrm{pTh}_{\mathrm{A}}^{(0)}(\bar{b})$ and $\bar{a} \models \varphi$. Following we will show that $\bar{b} \forall \varphi$. Suppose on the contrary that $\bar{b} \vDash \varphi$. Then there exists some $c \in A$ and a valuation $v: X \rightarrow A$ with $v\left(x_{i}\right)=f\left(b_{i}\right)(i=1, \ldots, m)$ such that

$$
\mathbf{A} \models_{v\left(x_{m+1} / c\right)} \psi .
$$

Since $\{\psi\}^{\prime}=\Phi^{\prime}$, this is equivalent to

$$
\forall \chi \in \Phi: \quad \mathbf{A} \models_{v\left(x_{m+1} / c\right)} \chi
$$

which means that for all $\varrho \in R$ and for all $i_{1}, \ldots, i_{\operatorname{ar}(\varrho)} \in\{1, \ldots, m+1\}$ with $\left(b_{i_{1}}, \ldots, b_{i_{\operatorname{ar}(\varrho)}}\right) \in \varrho_{\mathbf{A}}$ we have that

$$
\mathbf{A} \models_{v\left(x_{m+1} / c\right)} \varrho\left(x_{i_{1}}, \ldots, x_{i_{\text {ar(o) }}}\right) .
$$

Now define a mapping $g: \mathbf{B} \cup\{d\} \rightarrow \mathbf{A}$ in the following way:

$$
g(x)=\left\{\begin{aligned}
f(x), & \text { if } x \in B \\
\mathcal{c}, & \text { if } x=d
\end{aligned}\right.
$$

With this definition (1) can be rewritten as

$$
\forall \chi \in \Phi: \quad \mathbf{A} \models_{\left.x_{m+1} / g\left(b_{m+1}\right)\right)} \chi .
$$

We claim that $g$ is a homomorphism. For the proof, take $\varrho \in R$ and any tuple $\left(b_{i_{1}}, \ldots, b_{i_{\mathrm{ar}(\varrho)}}\right) \in \varrho_{A}$, where $\left\{i_{1}, \ldots, i_{\operatorname{ar}(\varrho)}\right\} \subseteq\{1, \ldots, m+1\}$. Then we know from $(2)$ that

$$
\mathbf{A} \models_{v\left(x_{m+1} / g\left(b_{m+1}\right)\right)} \varrho\left(x_{i_{1}}, \ldots, x_{i_{\text {ar }}}\right),
$$

i.e. $\left(g\left(b_{i_{1}}\right), \ldots, g\left(b_{i_{\text {ar }(\rho)}}\right)\right) \in \varrho_{\mathbf{A}}$, so $g$ is a homomorphism. Hence, $f$ can be extended to the homomorphism $g: \mathbf{B} \cup\{d\} \rightarrow \mathbf{A}$, which is a contradiction. Therefore, $\bar{b} \not \models \varphi$.

Proposition 1. Let $\mathbf{A}=\left(A,\left(\varrho_{A}\right)_{\varrho \in R}\right)$ be a weakly oligomorphic relational structure. Then $\mathbf{A}$ has the one-point-extension property if and only if $\mathbf{A}$ is endolocal.

Proof. $(\Rightarrow)$ We prove the claim by contraposition. Suppose that $\mathbf{A}$ is not endolocal. Then, since $\mathbf{A}$ is weakly oligomorphic, from 2.2.4(Proposition) it follows that there exist an $m \in \mathbb{N}$ and $\bar{c}, \bar{d} \in A^{m}$ such that $\mathrm{pTh}_{\mathbf{A}}^{(0)}(\bar{c}) \subseteq \operatorname{pTh}_{\mathbf{A}}^{(0)}(\bar{d})$, but $\operatorname{pTh}_{\mathbf{A}}(\bar{c}) \nsubseteq$ $\operatorname{pTh}_{\mathbf{A}}(\bar{d})$.

From this it follows that there exists a positive existential formula $\varphi$ over $R$, such that $\bar{c} \vDash \varphi$ and $\bar{d} \not \models \varphi$. Without loss of generality, $\varphi$ can be chosen of the shape

$$
\varphi \equiv \bigvee_{i \in I}\left(\exists x_{m+1}\right) \ldots\left(\exists x_{m+k}\right) \bigwedge_{j \in J_{i}} \varphi_{i j},
$$

where the $\varphi_{i j}$ are atomic formulae. This means that for every $i \in I$ we have that

$$
\bar{d} \not \models\left(\exists x_{m+1}\right) \ldots\left(\exists x_{m+k}\right) \bigwedge_{j \in J_{i}} \varphi_{i j}
$$


and that there exists an $\tilde{\imath} \in I$ such that

$$
\bar{c} \vDash\left(\exists x_{m+1}\right) \ldots\left(\exists x_{m+k}\right) \bigwedge_{j \in J_{i}} \varphi_{i j} .
$$

Then for the primitive positive formula

$$
\mu \equiv\left(\exists x_{m+1}\right) \ldots\left(\exists x_{m+k}\right) \bigwedge_{j \in J_{i}} \varphi_{i j}
$$

we have that $\bar{c} \models \mu$ and $\bar{d} \not \models \mu$.

By 0.2 .3 (Proposition), there exists a relational configuration $(\tilde{\mathbf{C}}, \tilde{\mathbf{D}}, \tilde{E}, \tilde{\theta})$ that corresponds to $\mu$ in $\mathbf{A}$. In particular $\bar{d} \Downarrow=(\tilde{\mathbf{C}}, \tilde{\mathbf{D}}, \tilde{E}, \tilde{\theta})$, and $\bar{c} \Vdash(\tilde{\mathbf{C}}, \tilde{\mathbf{D}}, \tilde{E}, \tilde{\theta})$. From the latter it follows that there exists a homomorphism $g: \tilde{\mathbf{D}} \rightarrow \mathbf{A}$ such that (G1) and (G2) from 0.2.3(Definition 1) hold.

The next step will be to strengthen $(\tilde{\mathbf{C}}, \tilde{\mathbf{D}}, \tilde{E}, \tilde{\theta})$. The resulting relational configuration should still have the property that $\bar{C}$ satisfies it and that $\bar{d}$ does not:

Construction of a strengthening of $(\tilde{\mathbf{C}}, \tilde{\mathbf{D}}, \tilde{E}, \tilde{\theta})$. Let $C, D$ be the carriers of $\tilde{\mathbf{C}}$, and $\tilde{\mathbf{D}}$, respectively. We can suppose that $D$ has $m+k$ elements. Let us enumerate the elements of $D$ by $\left\{y_{1}, y_{2}, \ldots y_{m+k}\right\}$ such that for all $1 \leq i \leq m$ we have that $E(i)=y_{i}$. This enumeration of $D$ we will denote by $F$ (i.e. $F(i)=y_{i}$ for $1 \leq i \leq m+k$ ).

Now we define an $R$-structure $\mathbf{D}=\left(D,\left(\varrho_{\mathbf{D}}\right)_{\varrho \in R}\right)$ by setting

$$
\varrho_{\mathbf{D}}:=\left\{\left(y_{i_{1}}, \ldots, y_{i_{\mathrm{ar}(\varrho)}}\right) \in D^{\operatorname{ar}(\varrho)} \mid\left(g\left(y_{i_{1}}\right), \ldots, g\left(y_{i_{\mathrm{ar}(\varrho)}}\right)\right) \in \varrho_{\mathbf{A}}\right\} .
$$

Moreover we define $\mathbf{C}$ to be the substructure of $\mathbf{D}$ that is induced by $\mathbf{C}$. Finally we set $E:=\tilde{E}$ and $\theta:=\operatorname{ker} g$. Next we will prove the following:

Claim 1. (C, D, $E, \theta)$ is stronger than $(\tilde{\mathbf{C}}, \tilde{\mathbf{D}}, \tilde{E}, \tilde{\theta})$ in $\mathbf{A}$,

Claim 2. $\bar{c} \Vdash(\tilde{\mathbf{C}}, \tilde{\mathbf{D}}, \tilde{E}, \tilde{\theta})$, but $\bar{d} \Downarrow=(\tilde{\mathbf{C}}, \tilde{\mathbf{D}}, \tilde{E}, \tilde{\theta})$.

proof of claim 1. Let $\bar{a} \in A^{m}$ such that $\bar{a} \|(\mathbf{C}, \mathbf{D}, E, \theta)$. This means that there exists a homomorphism $f: \mathbf{D} \rightarrow$ A with properties (G1) and (G2). We will show now that $\bar{a} \|(\tilde{\mathbf{C}}, \tilde{\mathbf{D}}, \tilde{E}, \tilde{\theta})$ by proving that $f: \tilde{\mathbf{D}} \rightarrow \mathbf{A}$ is a homomorphism that fulfills (G1) and (G2), relative to $(\tilde{\mathbf{C}}, \tilde{\mathbf{D}}, \tilde{E}, \tilde{\theta})$.

For this we take an arbitrary $\varrho \in R$ and $\left(y_{i_{1}}, \ldots, y_{i_{\text {ar }(\rho)}}\right) \in \varrho_{\tilde{\mathbf{D}}}$. By construction we have that $\varrho_{\tilde{\mathbf{D}}} \subseteq \varrho_{\mathbf{D}}$, so $\left(f\left(y_{i_{1}}\right), \ldots, f\left(y_{i_{\operatorname{ar}(\varrho)}}\right)\right) \in \varrho_{\mathbf{A}}$, and therefore, $f: \tilde{\mathbf{D}} \rightarrow \mathbf{A}$ is an homomorphism. Moreover, since $E=\tilde{E}$, we have that (G1) is fulfilled and because $\tilde{\theta} \subseteq \operatorname{ker} g=\theta \subseteq \operatorname{ker} f$, we also have that (G2) is fulfilled. Hence we have that $\bar{a} \|(\tilde{\mathbf{C}}, \tilde{\mathbf{D}}, \tilde{E}, \tilde{\theta})$.

proof of claim 2. From the fact that $(\mathbf{C}, \mathbf{D}, E, \theta)$ is stronger that $(\tilde{\mathbf{C}}, \tilde{\mathbf{D}}, \tilde{E}, \tilde{\theta})$ it follows at once that $\bar{d} \nVdash(\mathbf{C}, \mathbf{D}, E, \theta)$. In order to show that $\bar{c} \|(\mathbf{C}, \mathbf{D}, E, \theta)$ we consider once again the homomorphism $g: \tilde{\mathbf{D}} \rightarrow \mathbf{A}$ that was considered above. We note, that $\mathbf{D}$ was defined in a way such that also $g: \mathbf{D} \rightarrow \mathbf{A}$ is a homomorphism. Since $E=\tilde{E}$, we have that $g(E(i))=c_{i}$ for $1 \leq i \leq m$ and hence 
we have that (G1) is fulfilled. The property (G2) holds, too, because $\theta=\operatorname{ker} g$, by construction. This finishes the proof of claim 2 .

Construction of a sequence of relational configurations. Our next goal is to define a sequence of relational configurations $\left(\mathbf{D}_{\mathbf{i}}, \mathbf{D}_{\mathbf{i}}, E_{i}, \theta_{i}\right)$ for $0 \leq i \leq k$. For this we set

$$
D_{i}:=\left\{y_{1}, \ldots, y_{m+i}\right\} \quad E_{i}:=F \uparrow_{D_{i}} \quad \theta_{i}:=\theta \cap D_{i}^{2}
$$

In particular, we define $\mathbf{D}_{\mathbf{i}}$ to be the substructure of $\mathbf{D}$ that is induced by $D_{i}$.

If in addition for $0 \leq i \leq k$ we define

$$
g_{i}:=g \Gamma_{D_{i}} \text {, and } \bar{a}_{i}:=\left(g\left(y_{1}\right), \ldots, g\left(y_{m+i}\right)\right),
$$

then we see at once that $\bar{a}_{i} \|\left(\mathbf{D}_{\mathbf{i}}, \mathbf{D}_{\mathbf{i}}, E_{i}, \theta_{i}\right)$. On this point we note that $\bar{a}_{0}=\bar{c}$.

Sequences of homomorphisms $\tilde{g}_{i}: \mathbf{D}_{\mathbf{i}} \rightarrow \mathbf{A}$. Our next goal is to examine sequences of homomorphisms $\tilde{g}_{i}: \mathbf{D}_{i} \rightarrow \mathbf{A}$ with the following properties:

1. $\tilde{g}_{0}: y_{i} \mapsto d_{i}$ for $1 \leq i \leq m$,

2. $\tilde{g}_{i+1} \uparrow_{D_{i}}=\tilde{g}_{i}$ for $0 \leq i<k$,

3. $\theta_{i} \subseteq \operatorname{ker} \tilde{g}_{i}$ for $0 \leq i \leq k$.

Note that if we define $\bar{b}_{i}:=\left(\tilde{g}_{i}\left(y_{1}\right), \ldots, \tilde{g}_{i}\left(y_{m}+i\right)\right)$, then Properties 1 and 3 just say that $\bar{b}_{i} \|\left(\mathbf{D}_{\mathbf{i}}, \mathbf{D}_{\mathbf{i}}, E_{i}, \theta_{i}\right)$. Moreover, Property 1 says nothing else but that $\bar{b}_{0}=\bar{d}$.

The construction of such a sequence (if it exists at all), proceeds by induction on $i$. First we have to show that it will function for $i=0$ :

In this case the function $\tilde{g}_{0}$ is already prescribed by Property 1 , above. We still have to show that the mapping $\tilde{g}_{0}$ is a homomorphism and that $\theta_{0} \subseteq \operatorname{ker} \tilde{g}_{0}$ :

$\tilde{g}_{0}$ is a homomorphism: Consider the substructure $\mathbf{S}_{\overline{\mathbf{c}}}$ of $\mathbf{A}$ that is induced by $\left\{c_{1}, \ldots, c_{m}\right\}$. The mapping $g_{0}^{\prime}: D_{0} \rightarrow S_{\bar{c}}$ defined by $g_{0}^{\prime}: x \mapsto g_{0}(x)$, clearly is a homomorphism from $\mathbf{D}_{\mathbf{0}}$ to $\boldsymbol{S}_{\overline{\mathbf{c}}}$. Moreover, since $\operatorname{pTh}_{\mathbf{A}}^{(0)}(\bar{c}) \subseteq \operatorname{pTh}_{\mathbf{A}}^{(0)}(\bar{d})$, the mapping $h: S_{c} \rightarrow A$ that is defined by $h: c_{i} \mapsto d_{i}$, is a homomorphism from

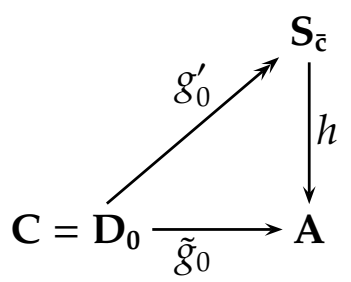

$\mathbf{S}_{\overline{\mathbf{c}}}$ to A. Now we note that $\tilde{g}_{0}=h \circ g_{0}^{\prime}$ (cf. diagram). Thus, indeed, $\tilde{g}_{0}$ is a homomorphism.

$\theta_{0} \subseteq \operatorname{ker} \tilde{g}_{0}$ : The claim that $\theta_{0} \subseteq \operatorname{ker} \tilde{g}_{0}$ follows directly from

$$
\theta_{0}=\operatorname{ker} g_{0}=\operatorname{ker} g_{0}^{\prime} \subseteq \operatorname{ker} h \circ g_{0}^{\prime}=\operatorname{ker} \tilde{g}_{0} \text {. }
$$

Let us now come to the key-step of the proof:

Key Step. We will now show that a complete sequence of homomorphisms $\tilde{g}_{i}$ for $0 \leq i \leq k$ with the above described three properties can in fact not exist. Indeed, if we suppose that a complete such sequence exists, then $\tilde{g}_{k}: \mathbf{D} \rightarrow \mathbf{A}$ has the 
properties $\tilde{g}_{k}\left(y_{i}\right)=d_{i}$ for $1 \leq i \leq m$ and that $\theta=\theta_{k} \subseteq$ ker $\tilde{g}_{k}$. This however proves that $\bar{d} \|(\mathbf{C}, \mathbf{D}, E, \theta)-$ a contradiction.

Now we argue that there has to exist a greatest $i_{0} \in\{0, \ldots, k-1\}$, such that homomorphisms $\tilde{g}_{i}$ with the above given three properties can be constructed for $0 \leq i \leq i_{0}$. In this case we know that there can not exist a homomorphism $\tilde{g}_{i_{0}+1}: \mathbf{D}_{\mathbf{i}_{0}+\mathbf{1}} \rightarrow \mathbf{A}$ with $\tilde{g}_{i_{0}+1}\left\lceil D_{i_{0}}=\tilde{g}_{i_{0}}\right.$ and with $\theta_{i_{0}+1} \subseteq \operatorname{ker} \tilde{g}_{i_{0}+1}$.

Let us fix a sequence $\tilde{g}_{i}$ for $0 \leq i \leq i_{0}$ with the above described three properties. By construction, we have that $\bar{b}_{i_{0}} \|\left(\mathbf{D}_{\mathbf{i}_{0}}, \mathbf{D}_{\mathbf{i}_{0}}, E_{i_{0}}, \theta_{i_{0}}\right)$.

We claim that $b_{i_{0}} \|=\left(\mathbf{D}_{\mathbf{i}_{0}}, \mathbf{D}_{\mathbf{i}_{0}+1}, E_{i_{0}}, \theta_{i_{0}+1}\right)$. Suppose, on the contrary, that $b_{i_{0}} \|$ $\left(\mathbf{D}_{\mathbf{i}_{0}}, \mathbf{D}_{\mathbf{i}_{\mathbf{0}}+\mathbf{1}}, E_{i_{0}}, \theta_{i_{0}+1}\right)$. Then there exists a homomorphism $\hat{g}: \mathbf{D}_{\mathbf{i}_{0}+\mathbf{1}} \rightarrow \mathbf{A}$ with Properties (G1) and (G2). The Property (G1) translates to $\hat{g} \uparrow_{D_{i_{0}}}=\tilde{g}_{i_{0}}$, and Property (G2) means that $\theta_{i_{0}+1} \subseteq \operatorname{ker} \hat{g}$. However, in this case $\hat{g}$ can be taken as $\tilde{g}_{i_{0}+1}$. This however contradicts the choice of $i_{0}$.. Thus, indeed, $b_{i_{0}} l \models\left(\mathbf{D}_{\mathbf{i}_{0}}, \mathbf{D}_{\mathbf{i}_{0}+1}, E_{i_{0}}, \theta_{i_{0}+1}\right)$.

Construction of a local homomorphism of A: We set $B:=\left\{g_{i_{0}}\left(y_{1}\right), \ldots, g_{i_{0}}\left(y_{m+i_{0}}\right)\right\}$, and $\mathbf{B}$ as the substructure of $\mathbf{A}$ induced by $B$. Moreover, we define the mapping $h: B \rightarrow A$ by $h\left(g_{i_{0}}\left(y_{l}\right)\right):=\tilde{g}_{i_{0}}\left(y_{l}\right)$. Since $\operatorname{ker} g_{i_{0}}=\theta_{i_{0}} \subseteq \operatorname{ker} \tilde{g}_{i_{0}}$, it follows that $h$ is well-defined. We claim that $h$ is a homomorphism from $\mathbf{B}$ to $\mathbf{A}$. To see this, take some $\varrho \in R$, and some $\bar{e} \in \varrho_{\mathbf{B}}$. Then, by the construction of $B$, there exist $l_{1}, \ldots, l_{\operatorname{ar}(\varrho)}$ such that

$$
\bar{e}=\left(g_{i_{0}}\left(y_{l_{1}}\right), \ldots, g_{i_{0}}\left(y_{l_{\text {ar }(\varrho)}}\right)\right) .
$$

Since $g_{i_{0}}=g \Gamma_{D_{i_{0}}}$ and since $\mathbf{D}_{\mathbf{i}_{0}} \leq \mathbf{D}$, and finally, because of the construction of $\varrho_{\mathbf{D}}$,we conclude that $\left(y_{l_{1}}, \ldots, y_{l_{\mathrm{ar}(\varrho)}}\right) \in \varrho_{\mathbf{D}_{\mathbf{i}_{0}}}=\varrho_{\mathbf{D}} \cap D_{i_{0}}^{2}$. With this we can compute

$$
h(\bar{e})=\left(\tilde{g}_{i_{0}}\left(y_{l_{1}}\right), \ldots, \tilde{g}_{i_{0}}\left(y_{l_{\operatorname{ar}(\varrho)}}\right)\right),
$$

and because $\tilde{g}_{i_{0}}$ is a homomorphism, we conclude that $h(\bar{e}) \in \varrho_{\mathbf{A}}$. It remains to show that $h$ can not be extended to $\mathbf{B} \cup\left\{g\left(y_{m+i_{0}+1}\right)\right\}$.

The non-extensibility of $h$. Suppose, that there exists a homomorphism $\hat{h}: \mathbf{B} \cup$ $\left\{g\left(y_{m+i_{0}+1}\right)\right\} \rightarrow \mathbf{A}$ that extends $h$. Then for $0 \leq i \leq k$ we define $g_{i}^{\#}$ to be the image-restriction of $g_{i}$ to $\left\{g_{i}\left(y_{1}\right), \ldots, g_{i}\left(y_{m+i}\right)\right\}$. We go on by defining $\tilde{g}_{i_{0}+1}:=\hat{h} \circ g_{i_{0}+1}^{\#}$ (cf.

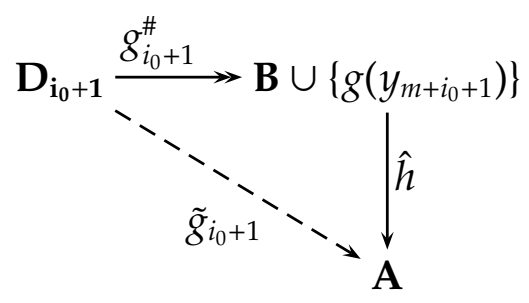
Diagram (3) $)$. By the definition of $h$ we have that $\tilde{g}_{i_{0}}=h \circ g_{i_{0}}^{\#}$. Hence $\tilde{g}_{i_{0}+1} \Upsilon_{D_{i_{0}}}=\tilde{g}_{i_{0}}$ (cf. Diagram (4)), and $\theta=\operatorname{ker} g_{i_{0}+1}=$ $\operatorname{ker} g_{i_{0}+1}^{\#} \subseteq \operatorname{ker} \hat{h} \circ g_{i_{0}+1}^{\#}=\operatorname{ker} \tilde{g}_{i_{0}+1}$.

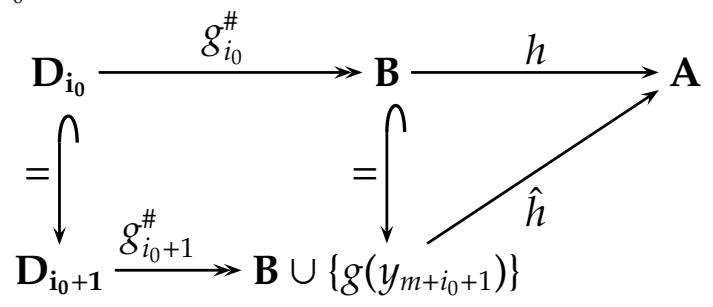


Thus we have an extension of the sequence of the $\tilde{g}_{i}$ by $\tilde{g}_{i_{0}+1}-$ contradiction. Hence $h$ can not be extended to $\mathbf{B} \cup\left\{g\left(y_{m+i_{0}+1}\right)\right\}$ and consequently, A does not have the one-point extension property.

$(\Leftarrow)$ We prove this claim indirectly. Suppose that $\mathbf{A}$ is endolocal but that it does not have the one-point extension property. Then, since $\mathbf{A}$ is weakly oligomorphic, by the previous Lemma, we obtain that there exist an $m \in \mathbb{N}$, an irreflexive $m$ tuple $\bar{a}$, an $m$-tuple $\bar{b}$ and a logical proposition $\varphi \equiv(\exists x) \psi$, where $\psi$ is a conjunction of atomic formulae, such that $\mathrm{pTh}^{(0)}(\bar{a}) \subseteq \mathrm{pTh}^{(0)}(\bar{b})$, and $\bar{a} \vDash \varphi$, but $\bar{b} \not \varphi$. This implies that $\mathrm{pTh}(\bar{a}) \nsubseteq \mathrm{pTh}(\bar{b})$, so by 2.2.3(Proposition 2), it follows that $A$ is not endolocal.

Yet another step in characterizing structures that are not endolocal is the use of a more relaxed version of the one-point extension property.

Definition 4. Given a relational structure $\mathbf{A}=\left(A,\left(\varrho_{\mathbf{A}}\right)_{\varrho \in R}\right)$ and its finite substructure $\mathbf{B}=\left(B,\left(\varrho_{\mathbf{B}}\right)_{\varrho \in R}\right)$. We say that a $c \in A$ is a weak center of $\mathbf{B}$ if

for every $b \in B$ there exists a $\varrho \in R, b_{3}, \ldots, b_{\operatorname{ar}(\varrho)} \in B$ and a $\pi \in \operatorname{Sym}\{1, \ldots, \operatorname{ar}(\varrho)\}$ such that

$$
\left(c, b, b_{3}, \ldots, b_{\operatorname{ar}(\varrho)}\right)^{\pi} \in \varrho_{\mathbf{A}} .
$$

Definition 5. We say that a relational structure $\mathbf{A}=\left(A,\left(\varrho_{A}\right)_{\varrho \in R}\right)$ has the weak one-point-extension property if for every finite substructure $\mathbf{B}$ of $\mathbf{A}$, every homomorphism $f: \mathbf{B} \rightarrow \mathbf{A}$ and every weak center $c$ of $\mathbf{B}$ there is a homomorphism $g: \mathbf{B} \cup\{c\} \rightarrow \mathbf{A}$ such that $g \uparrow_{B}=f$.

Proposition 2. A relational structure $\mathbf{A}$ has the one-point-extension property if and only if it has the weak one-point-extension property.

Proof. $(\Rightarrow)$ Obvious.

$(\Leftarrow)$ Let $\mathbf{A}$ have the weak one-point-extension property. We will show that then it also has the one-point-extension property.

For the proof, take a finite substructure $\mathbf{B}$ of $\mathbf{A}$, a homomorphism $f: \mathbf{B} \rightarrow \mathbf{A}$ and an $a \in A$. If $a$ is a weak center, then $f$ is extendable to $g: \mathbf{B} \cup\{a\} \rightarrow \mathbf{A}$. Otherwise, $a$ is not a weak center, meaning that there exists a $b \in B$ such that for every $\varrho \in R$, every $b_{3}, \ldots, b_{\operatorname{ar}(\varrho)} \in B$ and every $\pi \in \operatorname{Sym}\{1, \ldots, \operatorname{ar}(\varrho)\}$ holds that

$$
\left(a, b, b_{3}, \ldots, b_{\mathrm{ar}(\varrho)}\right)^{\pi} \notin \varrho_{\mathrm{A}} .
$$

Let $\tilde{B}$ be a set of all $b \in B$ that fulfill (6).

Case 1. Suppose $B=\tilde{B}$. This means that $a$ does not appear as an entry of any tuple from any relation $\varrho_{\mathbf{B} \cup\{a\}}$. So, we can choose an arbitrary element of $A$ for its image, say $a$. Then the mapping

$$
g(x)=\left\{\begin{array}{rr}
f(x), & \text { if } x \in B \\
a, & \text { if } x=a
\end{array}\right.
$$

is going to be a one-point-extension. 
Case 2. Suppose $B \backslash \tilde{B} \neq \emptyset$. Take an arbitrary $d \in B \backslash \tilde{B}$. Then $d$ fulfills the negation of Property (6), which is the negation of Property (5), so $d$ has Property (5), and, therefore, $a$ is a weak center of $\mathbf{B} \backslash \tilde{\mathbf{B}}$. It follows that we can extend $f \uparrow_{B \backslash \tilde{B}}$ to $h:(\mathbf{B} \backslash \tilde{\mathbf{B}}) \cup\{a\} \rightarrow \mathbf{A}$. We claim that in this case

$$
g(x)= \begin{cases}f(x), & \text { if } x \in B . \\ h(a), & \text { if } x=a .\end{cases}
$$

is going to be the requested one-point extension. It is clear that $g$ is well-defined, so it is left to show that it is a homomorphism. For the proof, take some $\varrho \in R$ and some $\left(d_{1}, \ldots, d_{\operatorname{ar}(\varrho)}\right) \in \varrho_{\mathbf{B} \cup\{a\}}$.

If $d_{1}, \ldots, d_{\operatorname{ar}(\varrho)} \in B$, then $\left(g\left(d_{1}\right), \ldots, g\left(d_{\operatorname{ar}(\varrho)}\right)\right)=\left(f\left(d_{1}\right), \ldots, f\left(d_{\operatorname{ar}(\varrho)}\right)\right) \in \varrho_{\mathbf{B}} \subseteq \varrho_{\mathbf{A}}$. Otherwise, there exists an $i \in\{1, \ldots, \operatorname{ar}(\varrho)\}$ such that $d_{i}=a$. But, then for every $j \in\{1, \ldots, \operatorname{ar}(\varrho)\} \backslash\{i\}$ we have that $d_{j} \in(B \backslash \tilde{B}) \cup\{a\}$, so $\left(g\left(d_{1}\right), \ldots, g\left(d_{\operatorname{ar}(\varrho)}\right)\right)=$ $\left(h\left(d_{1}\right), \ldots, h\left(d_{\operatorname{ar}(\varrho)}\right)\right) \in \varrho_{\mathbf{A}}$.

2.3.3. Homomorphism-homogeneous relational structures. In 2.3 .2 we derived the notion of weakly homomorphism-homogeneous structures from the well-known model theoretical notion of weakly homogeneous structures. Weak homogeneity is closely related to ultrahomogeneity. Our next goal is to see how weak homomorphism-homogeneity is related to homomorphism-homogeneity.

Definition. We call a structure D homomorphism-homogeneous if every local homomorphism of $\mathbf{D}$ extends to an endomorphism of $\mathbf{D}$.

Proposition 1. If a relational structure $\mathbf{A}$ is homomorphism-homogeneous, then it has the one-point extension property.

Proof. Let $\mathbf{A}$ be a homomorphism-homogeneous relational structure. Then every local homomorphism of $\mathbf{A}$ can be extended to an endomorphism of $\mathbf{A}$.

Take an arbitrary finite substructure $\mathbf{B}$ of $\mathbf{A}$ and a homomorphism $f: \mathbf{B} \rightarrow \mathbf{A}$. Then there is a $g \in \operatorname{End}(\mathbf{A})$ such that $g \uparrow_{B}=f$. Now choose any $b \in A \backslash B$. Then $g \uparrow_{B \cup\{b\}}$ is a homomorphism that extends $B$ by one point.

Proposition 2. If a finite or countably infinite relational structure $\mathbf{A}$ has the one-point extension property, then it is homomorphism-homogeneous.

Proof. Let A have the one-point extension property. Take a local homomorphism $f: \mathbf{B} \rightarrow \mathbf{A}$, where $\mathbf{B}$ is a finite substructure of $\mathbf{A}$.

The one-point extension property allows us to extend $f$ to $f_{1}: \mathbf{B} \cup\{b\} \rightarrow \mathbf{A}$, for arbitrary $b \in A \backslash B$. Since $A$ is a finite or countably infinite set, we can take a fixed well-ordering of $A$ that is isomorphic to an ordinal number $\alpha \leq \omega$, and then add to $B$ in every next step the smallest element from $A$ that was not yet added in the steps before. This defines a growing sequence of homomorphisms $f=g_{0} \subseteq g_{1} \subseteq g_{2} \subseteq \cdots$ (Here we identify the local homomorphisms with their graphs). The union of this sequence is an endomorphism of $\mathbf{A}$ that extends $f$. 


\subsection{Main Theorem}

In the previous sections of this chapter we studied the notion of endolocality. As a result of this study we obtained several characterizations of this notion using the language of formal concept analysis and model theory. Unfortunately, these results are scattered into many propositions with subtle side-conditions, so it is difficult to get the big picture. In the theorem that follows we collect all important data and give the overview.

Theorem. Let $\mathbf{A}=\left(A,\left(\varrho_{\mathbf{A}}\right)_{\varrho \in R}\right)$ be a relational structure. Then the relationships presented in the following diagram hold:

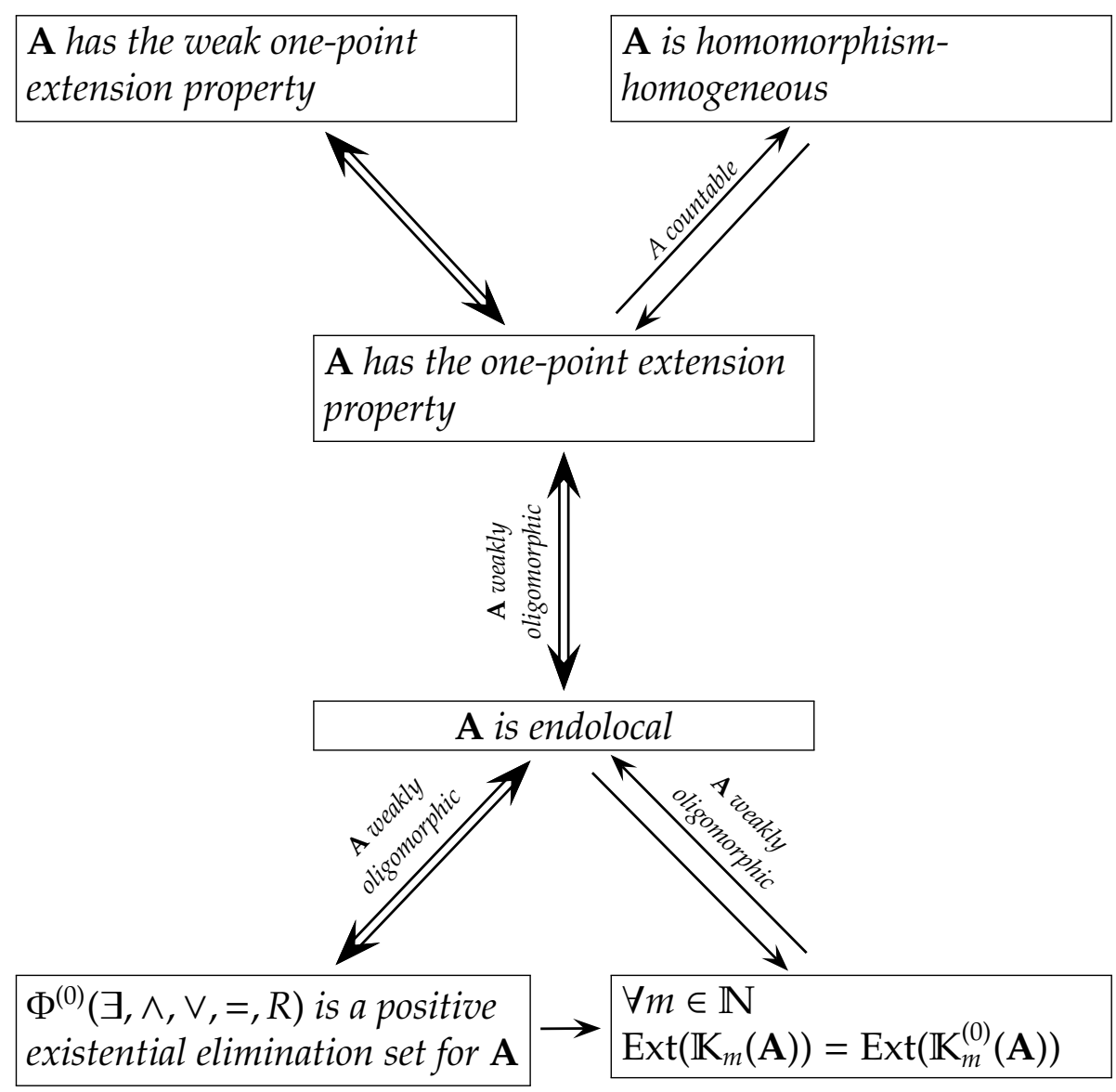

Proof. All of the given implications have already been proved in the previous sections. The table that follows gives the reference for each of the implications with the aim to make reading easier: 


\begin{tabular}{|c|c|c|c|c|c|c|}
\hline$\Rightarrow$ & Endolocality & $\begin{array}{l}\text { One-point ex- } \\
\text { tension }\end{array}$ & $\begin{array}{l}\text { Weak one- } \\
\text { point exten- } \\
\text { sion }\end{array}$ & $\begin{array}{l}\text { Homomor- } \\
\text { phism-homo- } \\
\text { geneity }\end{array}$ & $\begin{array}{l}\text { Positive } \\
\text { existential } \\
\text { elimination } \\
\text { set }\end{array}$ & Equal extents \\
\hline Endolocality & & $\begin{array}{c}2.3 .2 \\
\text { (Prop. 1) }\end{array}$ & & & $\begin{array}{c}2.3 .1 \\
\text { (Prop. 2) }\end{array}$ & $\begin{array}{l}2.2 .3 \\
\text { (Cor.) } \\
\end{array}$ \\
\hline $\begin{array}{l}\text { One-point ex- } \\
\text { tension }\end{array}$ & $\begin{array}{c}2.3 .2 \\
\text { (Prop. 1) }\end{array}$ & & $\begin{array}{c}2.3 .2 \\
\text { (Prop. 2) }\end{array}$ & $\begin{array}{c}2.3 .3 \\
\text { (Prop. 2) }\end{array}$ & & \\
\hline $\begin{array}{l}\text { Weak one- } \\
\text { point exten- } \\
\text { sion }\end{array}$ & & $\begin{array}{c}2.3 .2 \\
\text { (Prop. 2) } \\
\end{array}$ & & & & \\
\hline $\begin{array}{l}\text { Homomor- } \\
\text { phism-homo- } \\
\text { geneity }\end{array}$ & & $\begin{array}{c}2.3 .3 \\
\text { (Prop. 1) } \\
\end{array}$ & & & & \\
\hline $\begin{array}{l}\text { Positive } \\
\text { existential } \\
\text { elimination } \\
\text { set }\end{array}$ & $\begin{array}{c}2.3 .1 \\
\text { (Prop. 1) } \\
\end{array}$ & & & & & $\begin{array}{c}2.3 .1 \\
\text { (Rem.) }\end{array}$ \\
\hline Equal extents & $\begin{array}{l}2.2 .3 \\
\text { (Cor.) } \\
\end{array}$ & & & & & \\
\hline
\end{tabular}

In the Main theorem many implications hold under the additional condition that a structure in question is weakly oligomorphic. This property is not easily recognizable for a given relational structure. We will now show that the Main theorem is applicable to all relational structures with a finite signature.

Lemma. Let $\mathbf{A}=\left(A,\left(\varrho_{\mathbf{A}}\right)_{\varrho \in R}\right)$ be a relational structure with a finite signature $R$. Then for every $m \in \mathbb{N}$ there exists an $s \in \mathbb{N}$ such that for every $\bar{a} \in A^{m}$ there exists a $\varphi(\bar{a}) \in \operatorname{pTh}^{(s)}(\bar{a})$ such that $\left(\mathrm{pTh}_{\mathbf{A}}^{(k)}(\bar{a})\right)^{\prime}=(\varphi(\bar{a}))^{\prime}$ in $\mathbb{K}_{m}(\mathbf{A})$.

Proof. Since $\mathrm{pTh}_{\mathrm{A}}^{(k)}(\bar{a}) \subseteq \Phi_{m}^{(k)}(\exists, \wedge, \vee,=, R)$, using 0.2.2(Corollary) we obtain that $\mathrm{pTh}_{\mathrm{A}}^{(k)}(\bar{a})$ contains, up to equivalence, finitely many formulae. Let $\varphi_{1}, \varphi_{2}, \ldots, \varphi_{l}$ be a transversal of these equivalence classes. Then the formula

$$
\varphi(\bar{a}) \equiv \varphi_{1} \wedge \varphi_{2} \wedge \cdots \wedge \varphi_{l}
$$

is the desired formula. Indeed,

$$
\begin{aligned}
\bar{b} \in(\varphi(\bar{a}))^{\prime} & \Leftrightarrow \bar{b} \vDash \varphi(\bar{a}) \Leftrightarrow \forall i \in\{1, \ldots, l\}: \quad \bar{b} \models \varphi_{i} \\
& \Leftrightarrow \bar{b} \vDash \operatorname{pTh}_{\mathbf{A}}^{(k)}(\bar{a}) \Leftrightarrow \bar{b} \in\left(\operatorname{pTh}_{\mathbf{A}}^{(k)}(\bar{a})\right)^{\prime} .
\end{aligned}
$$

Moreover, $l \leq L$, where $L$ is the number of equivalence classes of formulae in $\Phi_{m}^{(k)}(\exists, \wedge, \vee,=, R)$, so the quantifier depth of $\varphi(\bar{a})$ is bounded from above by $s=L \cdot k$. 
Proposition. If $\mathbf{A}$ is a relational structure over a finite signature and if there exists some $k \in \mathbb{N}$ such that for every $m \in \mathbb{N}$ we have that $\operatorname{Ext}\left(\mathbb{K}_{m}(\mathbf{A})\right)=\operatorname{Ext}\left(\mathbb{K}_{m}^{(k)}(\mathbf{A})\right)$, then $\mathbf{A}$ is weakly oligomorphic.

Proof. We will show that every context $\mathbb{K}_{m}(\mathbf{A})$ has just finitely many row intents $\{\bar{a}\}^{\prime}, \bar{a} \in A^{m}$. By the previous Lemma, we know that for $m \in \mathbb{N}$ there exists an $s$ such that for all $\bar{a} \in A^{m}$, there is a $\varphi(\bar{a}) \in \operatorname{pTh}_{\mathbf{A}}^{(s)}(\bar{a})$ with $\{\bar{a}\}^{\prime}=(\varphi(\bar{a}))^{\prime \prime}$. Since there are up to equivalence just finitely many formulae in $\operatorname{pTh}_{\mathbf{A}}^{(s)}(\bar{a})$, there are up to equivalence just finitely many different intents of the shape $(\varphi(\bar{a}))^{\prime \prime}, \bar{a} \in A^{m}$. Hence, the cross-table of $\mathbb{K}_{m}^{(k)}(\mathbf{A})$ has just finitely many different rows. Thus, $\mathbb{K}_{m}^{(k)}(\mathbf{A})$ has just finitely many extents, and, hence, $\operatorname{Ext}\left(\mathbb{K}_{m}(\mathbf{A})\right)$ is finite for all $m$.

\subsection{Endolocality and weak Krasner algebras}

Every relational structure $\mathbf{A}=\left(A,\left(\varrho_{A}\right)_{\varrho \in R}\right)$ can be considered as a generating system of the weak Krasner algebra $\left[\left(\varrho_{A}\right)_{\varrho \in R}\right]_{\text {WKA }}$. Such a generating system is not unique, so all relational structures can be partitioned into equivalence classes according to the weak Krasner algebra that they define.

Definition. Let $\mathbf{A}=\left(A,\left(\varrho_{\mathbf{A}}\right)_{\varrho \in R}\right)$ and $\tilde{\mathbf{A}}=\left(A,\left(\varrho_{\tilde{\mathbf{A}}}\right)_{\varrho \in \tilde{R}}\right)$ be relational structures. We say that $\mathbf{A}$ and $\tilde{\mathbf{A}}$ are WKA-equivalent if $\left[\left(\varrho_{\mathbf{A}}\right)_{\varrho \in R}\right]_{\mathrm{WKA}}=\left[\left(\varrho_{\tilde{\mathbf{A}}}\right)_{\varrho \in \tilde{R}}\right]_{\mathrm{WKA}}$.

This gives us the opportunity to study weak Krasner algebras with respect to a generating relational structure. If the relational structure is endolocal and weakly oligomorphic, then such a choice of a generating system remarkably simplifies the understanding of the structure of the weak Krasner algebra in question. In the following we will give the arguments that support this thesis.

1. If we have an endolocal structure over a finite signature, then the $m$-ary part of its weak Krasner algebra can be effectively described.

2. If we have a weakly oligomorphic homomorphism-homogeneous relational structure $\mathbf{A}=\left(A,\left(\varrho_{A}\right)_{\varrho \in R}\right)$, then the weak Krasner algebra defined by this structure is locally closed, that is

$$
\operatorname{Inv} \operatorname{End}(\mathbf{A})=\left[\left(\varrho_{\mathbf{A}}\right)_{\varrho \in R}\right]_{\mathrm{WKA}} \text {. }
$$

3. For a finite endolocal relational structure A over a finite signature it is easy to test whether a given relation is contained in the weak Krasner algebra described by $\mathbf{A}$.

Remark. Note that the property of being weakly oligomorphic for relational structures is stable under WKA-equivalence, that is, if a relational structure $\mathbf{A}$ is weakly oligomorphic, then every relational structure $\tilde{\mathbf{A}}$ that is WKA-equivalent to $\mathbf{A}$ is weakly oligomorphic, too. 
2.5.1. About argument 1, Let $\mathbf{A}=\left(A,\left(\varrho_{\mathbf{A}}\right)_{\varrho \in R}\right)$ be an endolocal relational structure over a finite signature $R$. We define a type function type for $_{\mathbf{A}} \mathbf{A}$ and $m \in \mathbb{N}$ as follows:

$$
\text { for } \bar{a} \in A^{m} \quad: \quad \operatorname{type}_{\mathbf{A}}(\bar{a}):=\left\{\varphi \in \Phi_{m}(\exists, \wedge, \vee,=, R) \mid \varphi \text { atom, } \bar{a} \vDash \varphi\right\} .
$$

As the order on types we take the usual inclusion.

Proposition 1. The type function type ${ }_{\mathbf{A}}$ defines by the Locality Principle the closure system of extents of $\mathbb{K}_{m}(\mathbf{A})$.

Proof. It suffices to show that for $\bar{a}, \bar{b} \in A^{m}$

$$
\text { type }_{\mathbf{A}}(\bar{a}) \sqsubseteq \operatorname{type}_{\mathbf{A}}(\bar{b}) \text { if and only if } \operatorname{pTh}_{\mathbf{A}}^{(0)}(\bar{a}) \subseteq \operatorname{pTh}_{\mathbf{A}}^{(0)}(\bar{b}) .
$$

Denote by $\operatorname{At}_{m}(R)$ the set of all atoms in $\Phi_{m}(\exists, \wedge, \vee,=, R)$ and suppose that $\operatorname{pTh}_{\mathbf{A}}^{(0)}(\bar{a}) \subseteq \operatorname{pTh}_{\mathbf{A}}^{(0)}(\bar{b})$. Then

$$
\operatorname{type}_{\mathbf{A}}(\bar{a})=\operatorname{pTh}_{\mathbf{A}}^{(0)}(\bar{a}) \cap \operatorname{At}_{m}(R) \subseteq \operatorname{pTh}_{\mathbf{A}}^{(0)}(\bar{b}) \cap \operatorname{At}_{m}(R)=\operatorname{type}_{\mathbf{A}}(\bar{b}) .
$$

For the other direction, suppose that $\operatorname{type}_{\mathbf{A}}(\bar{a}) \sqsubseteq \operatorname{type}_{\mathbf{A}}(\bar{b})$. Let, further, $\varphi \in$ $\operatorname{pTh}_{\mathbf{A}}^{(0)}(\bar{a})$. Then $\varphi$ is of the shape

$$
\varphi \equiv \bigvee_{i \in I} \bigwedge_{j \in J_{i}} \varphi_{i j}
$$

where $\varphi_{i j}$ are atoms. We know that $\bar{a} \vDash \varphi$, so $\bar{a} \vDash \bigvee_{i \in I} \bigwedge_{j \in J_{i}} \varphi_{i j}$. Then there exists an $i \in I$ such that $\bar{a} \vDash \bigwedge_{j \in J_{i}} \varphi_{i j}$, so it follows that for every $j \in J_{i}$ : $\bar{a} \vDash \varphi_{i j}$. By the assumption, $\bar{b} \vDash \varphi_{i j}$ for every $j \in J_{i}$, so $\bar{b} \vDash \bigwedge_{j \in J_{i}} \varphi_{i j}$, and, therefore, $\bar{b} \vDash \bigvee_{i \in I} \bigwedge_{j \in J_{i}} \varphi_{i j}$, implying that $\varphi \in \operatorname{pTh}_{\mathbf{A}}^{(0)}(\bar{b})$.

For a finite relational signature $R$, there can be only finitely many different atoms, and, therefore, finitely many different possible images of type ${ }_{\mathrm{A}}$. The realizable types correspond to join-irreducible elements in $\operatorname{Ext}\left(K_{m}(\mathbf{A})\right)$ (cf. 2.1.2(Proposition 2)). Since $\mathbf{A}$ is endolocal and $R$ is finite, by the Main Theorem and 2.4(Proposition), it follows that $\mathbf{A}$ is weakly oligomorphic, so $\left[\left(\varrho_{\mathbf{A}}\right)_{\varrho \in R}\right]_{\text {WKA }}^{(m)}=$ $\operatorname{Ext}\left(\mathbb{K}_{m}(\mathbf{A})\right)$ and we are then able to describe the $m$-ary part of the given weak Krasner algebra.

However, it is left to describe a method for deciding whether a given type is realizable. For this purpose, let $t \mathrm{p} \subseteq \mathrm{At}_{m}(R)$ be a type. We are asking whether there exists an $\bar{a} \in A^{m}$ such that tp $=\operatorname{type}_{\mathrm{A}}(\bar{a})$. In order to answer this question, we consider the relational structure $\mathbf{B}=\left(B,\left(\varrho_{\mathbf{B}}\right)_{\varrho \in R}\right)$, where $B:=\left\{x_{1}, \ldots, x_{m}\right\}$ and $\varrho_{\mathbf{B}}:=\left\{\left(x_{i_{1}}, \ldots, x_{i_{\mathrm{ar}(\varrho)}}\right) \mid \varrho\left(x_{i_{1}}, \ldots, x_{i_{\mathrm{ar}(\varrho)}}\right) \in \operatorname{tp}\right\}$. Additionally, we define on $B$ relation $\theta$ by

$$
\theta:=\left\{\left(x_{i}, x_{j}\right) \mid\left(x_{i}=x_{j}\right) \in t p\right\}
$$


If $\theta$ is not an equivalence relation, then tp is not realizable. From now on, we can assume that $\theta$ is an equivalence relation. In the next step we factorize $\mathbf{B}$ by $\theta$ and obtain $\tilde{\mathbf{B}}=\left(B / \theta,\left(\varrho_{\tilde{\mathbf{B}}}\right)_{\varrho \in R}\right)$, where $\varrho_{\tilde{\mathbf{B}}}:=\left\{\left(\left[x_{i_{1}}\right]_{\theta}, \ldots,\left[x_{i_{\operatorname{ar}(\varrho)}}\right]_{\theta}\right) \mid\left(x_{i_{1}}, \ldots, x_{i_{\operatorname{ar}(\varrho)}}\right) \in \varrho_{\mathbf{B}}\right\}$. Further, let $\chi_{\theta}$ be the natural homomorphism from $\mathbf{B}$ to $\tilde{\mathbf{B}}$. If tp is realizable, then $\chi_{\theta}$ is a full homomorphism, i.e. for all $\varrho \in R$ and all $\left(x_{i_{1}}, \ldots, x_{i_{\operatorname{ar}(\varrho)}}\right) \in B^{\operatorname{ar}(\varrho)}$ we have

$$
\left(x_{i_{1}}, \ldots, x_{i_{\operatorname{ar}(\varrho)}}\right) \in \varrho_{\mathbf{B}} \Leftrightarrow\left(\left[x_{i_{1}}\right]_{\theta}, \ldots,\left[x_{i_{\operatorname{ar}(\varrho)}}\right]_{\theta}\right) \in \varrho_{\tilde{\mathbf{B}}} .
$$

Proposition 2. A type tp is realizable if and only if

1. $\theta$ is an equivalence relation on $B$;

2. $\chi_{\theta}$ is a full homomorphism;

3. $\tilde{\mathbf{B}}$ is embeddable into $\mathbf{A}$.

Proof. $(\Rightarrow)$ Suppose tp is realizable. Then there exists an $\bar{a}=\left(a_{1}, \ldots, a_{m}\right) \in A^{m}$ such that $\mathrm{tp}=\operatorname{type}_{\mathbf{A}}(\bar{a})$. It is clear that $\theta$ is en equivalence relation on $B$ and that $\chi_{\theta}$ is a full homomorphism. We define the mapping $\iota: B / \theta \rightarrow A$ by

$$
\iota\left(\left[x_{i}\right]_{\theta}\right):=a_{i}, \quad i \in\{1, \ldots, m\} .
$$

and we claim that this is an embedding of $\tilde{\mathbf{B}}$ into $\mathbf{A}$.

$\iota$ is well-defined and injective:

$$
\begin{aligned}
{\left[x_{i}\right]_{\theta}=\left[x_{j}\right]_{\theta} } & \Leftrightarrow\left(x_{i}, x_{j}\right) \in \theta \Leftrightarrow\left(x_{i}=x_{j}\right) \in \operatorname{tp}=\operatorname{type}_{\mathbf{A}}(\bar{a}) \\
& \Leftrightarrow a_{i}=a_{j} \Leftrightarrow \iota\left(\left[x_{i}\right]_{\theta}\right)=\iota\left(\left[x_{j}\right]_{\theta}\right)
\end{aligned}
$$

$\iota$ is a full homomorphism:

$$
\begin{aligned}
\left(\left[x_{i_{1}}\right]_{\theta}, \ldots,\left[x_{i_{\operatorname{ar}(\varrho)}}\right]_{\theta}\right) \in \varrho_{\tilde{\mathbf{B}}} & \Leftrightarrow\left(x_{i_{1}}, \ldots, x_{i_{\operatorname{ar}(\varrho)}}\right) \in \varrho_{\mathbf{B}} \\
& \Leftrightarrow \varrho\left(x_{i_{1}}, \ldots, x_{i_{\operatorname{ar}(\varrho)}}\right) \in \operatorname{tp}=\operatorname{type}_{\mathbf{A}}(\bar{a}) \\
& \Leftrightarrow\left(a_{i_{1}}, \ldots, a_{i_{\operatorname{ar}(\varrho)}}\right) \in \varrho_{\mathbf{A}} .
\end{aligned}
$$

$(\Leftarrow)$ Suppose that $\theta$ is en equivalence relation on $B$ and that $\chi_{\theta}$ is a full homomorphism. Let, further, $\tilde{\mathbf{B}}$ be embeddable into $\mathbf{A}$ and let $\iota$ be such an embedding. We define

$$
\bar{a}=\left(a_{1}, \ldots, a_{m}\right):=\left(\iota\left(\left[x_{i_{1}}\right]_{\theta}\right), \ldots, \iota\left(\left[x_{i_{\operatorname{ar}(\varrho)}}\right]_{\theta}\right)\right) .
$$

We will show that tp $=\operatorname{type}_{\mathbf{A}}(\bar{a})$. For this we have to distinguish two cases:

Case 1.

$$
\begin{aligned}
\varrho\left(x_{i_{1}}, \ldots, x_{i_{\operatorname{ar}(\varrho)}}\right) \in \operatorname{type}_{\mathbf{A}}(\bar{a}) & \Leftrightarrow\left(a_{i_{1}}, \ldots, a_{i_{\operatorname{ar}(\varrho)}}\right) \in \varrho_{\mathbf{A}} \Leftrightarrow\left(\left[x_{i_{1}}\right]_{\theta}, \ldots,\left[x_{i_{\operatorname{ar}(\varrho)}}\right]_{\theta}\right) \in \varrho_{\tilde{\mathbf{B}}} \\
& \Leftrightarrow\left(x_{i_{1}}, \ldots, x_{i_{\operatorname{ar}(\varrho)}}\right) \in \varrho_{\mathbf{B}} \Leftrightarrow \varrho\left(x_{i_{1}}, \ldots, x_{i_{\operatorname{ar}(\varrho)}}\right) \in \operatorname{tp} .
\end{aligned}
$$

\section{Case 2.}

$$
\left(x_{i}=x_{j}\right) \in \operatorname{type}_{\mathbf{A}}(\bar{a}) \Leftrightarrow a_{i}=a_{j} \Leftrightarrow\left[x_{i}\right]_{\theta}=\left[x_{j}\right]_{\theta} \Leftrightarrow\left(x_{i}, x_{j}\right) \in \theta \Leftrightarrow\left(x_{i}=x_{j}\right) \in \operatorname{tp} .
$$


The process of deciding whether tp is realizable goes then in three steps. In the first step, we check if $\theta$ is an equivalence relation. This problem is easily decidable, since $B$ is finite. In the case of a positive answer, the next step is to compute $\tilde{\mathbf{B}}$ and to test whether $\chi_{\theta}$ is a full homomorphism. Again, this is possible, because of the finiteness of $\mathbf{B}$ and $\tilde{\mathbf{B}}$. If this test also succeeds, then it remains to examine whether $\tilde{\mathbf{B}}$ is isomorphic to a substructure of $\mathbf{A}$. Depending on $\mathbf{A}$, this problem may or may not be decidable. However, we can decide it for all finite structures and for many interesting infinite ones (e.g. chains, universal structures, etc.). Note that the class of all finite structures that can be embedded into $\mathbf{A}$ in model-theoretic terms is called the age of $\mathbf{A}$.

2.5.2. About argument 2, We will now show that if we have a weakly oligomorphic homomorphism-homogeneous relational structure $\mathbf{A}=\left(A,\left(\varrho_{A}\right)_{\varrho \in R}\right)$ then the weak Krasner algebra defined by this structure is locally closed, that is

$$
\operatorname{Inv} \operatorname{End}(\mathbf{A})=\left[\left(\varrho_{\mathbf{A}}\right)_{\varrho \in R}\right]_{W K A} .
$$

Instead of directly showing(1), we will show that for every $m \in \mathbf{N}$

$$
(\operatorname{Inv} \operatorname{End}(\mathbf{A}))^{(m)}=\left[\left(\varrho_{\mathbf{A}}\right)_{\varrho \in R}\right]_{\text {WKA }}^{(m)} .
$$

Since $\mathbf{A}$ is a weakly oligomorphic, homomorphism-homogeneous relational structure from the Main Theorem it follows that $\mathbf{A}$ is endolocal. As we have already seen in 2.5.1, for weakly oligomorphic endolocal relational structures, we have that $\left[\left(\varrho_{\mathbf{A}}\right)_{\varrho \in R}\right]_{\text {WKA }}^{(m)}=\operatorname{Ext}\left(\mathbb{K}_{m}(\mathbf{A})\right)$. Now it is enough to show that

$$
\forall \bar{a} \in A^{m}: \quad\{\bar{a}\}^{\prime \prime}=\Gamma_{\operatorname{End}(\mathbf{A})}(\{\bar{a}\}) .
$$

Indeed, if this equality holds, then for every relation $\sigma \in(\operatorname{Inv} \operatorname{End}(\mathbf{A}))^{(m)}$ we have that

$$
\sigma=\bigcup_{\bar{a} \in \sigma} \Gamma_{\operatorname{End}(\mathbf{A})}(\{\bar{a}\})=\bigcup_{\bar{a} \in \sigma}\{\bar{a}\}^{\prime \prime} \in \operatorname{Ext}\left(\mathbb{K}_{m}(\mathbf{A})\right)=\left[\left(\varrho_{\mathbf{A}}\right)_{\varrho \in R}\right]_{\mathrm{WKA}}^{(m)}
$$

since $\bigcup_{\bar{a} \in \sigma}\{\bar{a}\}^{\prime \prime}$ is, in fact, a finite union.

Let us now proceed by proving $[2)$. First of all, since $\{\bar{a}\}^{\prime \prime} \in\left[\left(\varrho_{\mathbf{A}}\right)_{\varrho \in R}\right]_{\text {WKA }}^{(m)} \subseteq$ $(\operatorname{Inv} \operatorname{End}(\mathbf{A}))^{(m)}$, it follows that $\Gamma_{\operatorname{End}(\mathbf{A})}(\{\bar{a}\}) \subseteq\{\bar{a}\}^{\prime \prime}$. On the other hand, for $\bar{b} \in\{\bar{a}\}^{\prime \prime}$ we have that $\bar{b} \vDash \mathrm{pTh}_{\mathbf{A}}(\bar{a})$. In particular, $\bar{b} \vDash \mathrm{pTh}_{\mathbf{A}}^{(0)}(\bar{a})$. Let $\bar{a}=\left(a_{1}, \ldots, a_{m}\right)$. We define the mapping $f:\left\{a_{1}, \ldots, a_{m}\right\} \rightarrow A$ by $f\left(a_{i}\right):=b_{i}$, for $i \in\{1, \ldots, m\}$. It is easy to see that this is a local homomorphism that maps $\bar{a}$ to $\bar{b}$. Since A is homomorphismhomogeneous, it follows that $f$ can be extended to an endomorphism $g$ of A. But, from this it follows at once that $\bar{b} \in \Gamma_{\operatorname{End}(\mathbf{A})}(\{\bar{a}\})$.

2.5.3. About argument 3. Suppose that we have given a finite endolocal relational structure $\mathbf{A}=\left(A,\left(\varrho_{\mathbf{A}}\right)_{\varrho \in R}\right)$ over a finite signature.

Let $\sigma \subseteq A^{m}$ be a given relation. We know that $\left[\left(\varrho_{\mathbf{A}}\right)_{\varrho \in R}\right]_{\mathrm{WKA}}^{(m)}=\operatorname{Ext}\left(\mathbb{K}_{m}(\mathbf{A})\right)$. Since A is endolocal, we have a few ways to test whether $\sigma \in \operatorname{Ext}\left(\mathbb{K}_{m}(\mathbf{A})\right)$ : 
1. We check whether $\sigma=\sigma^{\prime \prime}$. For this we use the Locality Principle (1.1) and the type-function that was given in 2.5.1.

2. From the Main Theorem it follows that $\operatorname{Ext}\left(\mathbb{K}_{m}(\mathbf{A})\right)=\operatorname{Ext}\left(\mathbb{K}_{m}^{(0)}(\mathbf{A})\right)$, so we have to check whether $\sigma \in \operatorname{Ext}\left(\mathbb{K}_{m}^{(0)}(\mathbf{A})\right)$. Of course, $\mathbb{K}_{m}^{(0)}(\mathbf{A})$ is not a finite context, but it can be reduced to a finite one in the following way:

Every formula from $\Phi_{m}^{(0)}(\exists, \wedge, \vee,=, R)$ can be written as a conjunction of disjunctions of atoms from $\operatorname{At}_{m}(R)$. On the other hand, every formula of the shape

$$
\psi \equiv \bigwedge_{i \in I} \varphi_{i}
$$

is reducible, because $\{\psi\}^{\prime}=\left\{\varphi_{i} \mid i \in I\right\}^{\prime}$, so we have just to consider disjunctions of atoms. Since $R$ is finite, there are just finitely many atoms, and, hence, finitely many disjunctions of them. So, for our new context, we take just those columns from $\mathbb{K}_{m}^{(0)}(\mathrm{A})$ that correspond to disjunctions of atoms.

2.5.4. Weak Krasner algebras generated by endolocal structures. In the previous we have argued that weak Krasner algebras generated by weakly oligomorphic and endolocal relational structures have several interesting properties. This opens the question of the characterization of weak Krasner algebras that are generated by such structures. In the following we will study this question.

Proposition 1. Let $\mathbf{A}=\left(A,\left(\varrho_{\mathbf{A}}\right)_{\varrho \in R}\right)$ be a relational structure and let $\Psi$ be a positive existential elimination set for A. Define $\tilde{R}:=\cup_{m \in \mathbb{N}}\left\{[\varphi] \mid \varphi \in \Psi \cap \Phi_{m}(\exists, \wedge, \vee,=, R)\right\}$ and $\tilde{\mathbf{A}}:=\left(A,\left([\varphi]_{\tilde{\mathbf{A}}}\right)_{[\varphi] \in \tilde{R}}\right)$, where $[\varphi]_{\tilde{\mathbf{A}}}:=\varphi^{\mathbf{A}}$. Then

1. $\mathbf{A}$ and $\tilde{\mathbf{A}}$ are WKA-equivalent, and

2. $\Phi^{(0)}(\exists, \wedge, \vee,=, \tilde{R})$ is a positive existential elimination set for $\tilde{\mathbf{A}}$.

Proof. About 1. Let $\sigma \in\left[\left(\varrho_{\mathbf{A}}\right)_{\varrho \in R}\right]_{\mathrm{WKA}}^{(m)}$. Then there exists a $\varphi \in \Phi_{m}(\exists, \wedge, \vee,=, R)$ such that $\varphi^{\mathbf{A}}=\sigma$. On the other hand,

$$
\varphi^{\mathbf{A}}=[\varphi]_{\tilde{\mathbf{A}}} \in\left[\left([\varphi]_{\tilde{\mathbf{A}}}\right)_{[\varphi] \in \tilde{R}}\right]_{\mathrm{WKA}}^{(m)} .
$$

For the other direction, let $\left.\sigma \in\left[\left([\varphi]_{\tilde{\mathbf{A}}}\right)_{[\varphi] \in \tilde{R}}\right)\right]_{\mathrm{WKA}}^{(m)}$. Then there is a $\tilde{\varphi} \in \Phi_{m}(\exists, \wedge, \vee,=$ $, \tilde{R})$ such that $\tilde{\varphi}^{\tilde{\mathrm{A}}}=\sigma$.

We claim that for every $\tilde{\psi} \in \Phi_{m}(\exists, \wedge, \vee,=, \tilde{R})$ there exists a $\psi \in \Phi_{m}(\exists, \wedge, \vee,=, R)$ such that $\tilde{\psi}^{\tilde{\mathbf{A}}}=\psi^{\mathbf{A}}$, and we prove it by structural induction.

If $\tilde{\psi}$ is an atomic formula then either

$$
\begin{gathered}
\tilde{\psi} \equiv\left(x_{i}=x_{j}\right), \text { where } i, j \leq m, \text { or } \\
\tilde{\psi} \equiv[\mu]\left(x_{i_{1}}, \ldots, x_{i_{k}}\right), \text { where } k=\operatorname{ar}([\mu]) \text { and }\left\{i_{1}, \ldots, i_{k}\right\} \subseteq\{1, \ldots, m\}
\end{gathered}
$$


In the first case, $\psi \equiv\left(x_{i}=x_{j}\right)$. In the second one, we obtain

$$
\begin{aligned}
\left([\mu]\left(x_{i_{1}}, \ldots, x_{i_{k}}\right)\right)^{\tilde{\mathbf{A}}} & =\left\{\left(a_{1}, \ldots, a_{m}\right) \mid\left(a_{i_{1}}, \ldots, a_{i_{k}}\right) \in[\mu]_{\tilde{\mathbf{A}}}\right\} \\
& =\left\{\left(a_{1}, \ldots, a_{m}\right) \mid\left(a_{i_{1}}, \ldots, a_{i_{k}}\right) \vDash_{\mathbf{A}} \mu\right\} \\
& =\left(\mu\left(x_{i_{1}}, \ldots, x_{i_{k}}\right)\right)^{\mathbf{A}},
\end{aligned}
$$

so $\psi \equiv \mu\left(x_{i_{1}}, \ldots, x_{i_{k}}\right)$.

If $\tilde{\psi}$ is not an atomic formula then either

$$
\tilde{\psi} \equiv \bigwedge_{i \in I} \tilde{\psi}_{i} \text { or } \tilde{\psi} \equiv \bigvee_{i \in I} \tilde{\psi}_{i} \text { or } \tilde{\psi} \equiv\left(\exists x_{k}\right) \tilde{\psi}_{1}
$$

where $I$ is finite, $k>m$ and for every $\tilde{\psi}_{i} \in \Phi_{m}(\exists, \wedge, \vee,=, \tilde{R})$ exists a $\psi_{i} \in$ $\Phi_{m}(\exists, \wedge, \vee,=, R)$ such that $\tilde{\psi}_{i}^{\tilde{\mathbf{A}}}=\psi_{i}^{\mathbf{A}}$. In the first case, we can take $\psi \equiv \bigwedge_{i \in I} \psi_{i}$. Indeed,

$$
\tilde{\psi}^{\tilde{\mathbf{A}}}=\left(\bigwedge_{i \in I} \tilde{\psi}_{i}\right)^{\tilde{\mathbf{A}}}=\bigcap_{i \in I} \tilde{\psi}_{i}^{\tilde{\mathbf{A}}}=\bigcap_{i \in I} \psi_{i}^{\mathbf{A}}=\left(\bigwedge_{i \in I} \psi_{i}\right)^{\mathbf{A}} .
$$

Analogously, in the second case we can take $\psi \equiv \bigvee_{i \in I} \psi_{i}$. In the last case, we can take $\psi \equiv\left(\exists x_{k}\right) \psi_{1}$. Then $\left(a_{1}, \ldots, a_{m}\right) \in \tilde{\psi}_{1}^{\tilde{\mathbf{A}}}$ is equivalent to the fact that there exists an $a \in A$ such that for some valuation $v: X \rightarrow A$ with $v\left(x_{i}\right)=a_{i}, i=1, \ldots, m$ holds $\tilde{\mathbf{A}} \models_{v\left(x_{k} / a\right)} \tilde{\psi}_{1}$. This is, however, by the inductional hypothesis equivalent to the fact that there exists an $a \in A$ such that for some valuation $v: X \rightarrow A$ with $v\left(x_{i}\right)=a_{i}, i=1, \ldots, m$ holds $\mathbf{A} \models_{v\left(x_{k} / a\right)} \psi_{1}$, i.e. $\left(a_{1}, \ldots, a_{m}\right) \in \psi_{1}{ }^{\mathbf{A}}$.

Thus, there exists a $\varphi \in \Phi_{m}(\exists, \wedge, \vee,=, R)$ such that $\sigma=\tilde{\varphi}^{\tilde{\mathbf{A}}}=\varphi^{\mathbf{A}}$, and the other inclusion also holds.

About 2. Let $\tilde{\varphi} \in \Phi_{m}(\exists, \wedge, \vee,=, \tilde{R})$. Then, as shown above, there exists a $\varphi \in \Phi_{m}(\exists, \wedge, \vee,=, R)$ such that $\tilde{\varphi}^{\tilde{\mathbf{A}}}=\varphi^{\mathbf{A}}$. But, then there exist $\psi_{i j} \in \Psi$ such that

$$
\varphi^{\mathbf{A}}=\left(\bigvee_{i \in I} \bigwedge_{j \in J_{i}} \psi_{i j}\right)^{\mathbf{A}} .
$$

For each $\psi_{i j}$ of arity $k_{i j}$ take $\left[\psi_{i j}\right]\left(x_{1}, \ldots, x_{k_{i j}}\right)$ and note that

$$
\psi_{i j}^{\mathbf{A}}=\left(\left[\psi_{i j}\right]\left(x_{1}, \ldots, x_{k_{i j}}\right)\right)^{\tilde{\mathbf{A}}}=\left[\psi_{i j}\right]_{\mathbf{A}} .
$$

Then we obtain

$$
\tilde{\varphi}^{\tilde{\mathbf{A}}}=\left(\bigvee_{i \in I} \bigwedge_{j \in J_{i}} \psi_{i j}\right)^{\mathbf{A}}=\left(\bigvee_{i \in I} \bigwedge_{j \in J_{i}}\left[\psi_{i j}\right]\left(x_{1}, \ldots, x_{k_{i j}}\right)\right)^{\tilde{\mathbf{A}}},
$$

so $\tilde{\varphi} \equiv \bigvee_{i \in I} \bigwedge_{j \in J_{i}}\left[\psi_{i j}\right]\left(x_{1}, \ldots, x_{k_{i j}}\right) \in \Phi^{(0)}(\exists, \wedge, \vee,=, \tilde{R})$, and therefore, $\Phi^{(0)}(\exists, \wedge, \vee,=$ $, \tilde{R})$ is a positive existential elimination set for $\tilde{\mathbf{A}}$. 
Corollary 1. For every relational structure $\mathbf{A}=\left(A,\left(\varrho_{\mathbf{A}}\right)_{\varrho \in R}\right)$ there exists a WKAequivalent relational structure $\tilde{\mathbf{A}}=\left(A,\left(\varrho_{\tilde{\mathbf{A}}}\right)_{\varrho \in \tilde{R}}\right)$ such that $\Phi^{(0)}(\exists, \wedge, \vee,=, \tilde{R})$ is a positive existential elimination set for $\tilde{\mathbf{A}}$.

Proof. Clearly, $\Phi(\exists, \wedge, \vee,=, R)$ is a positive existential elimination set of every $R$-structure A. Hence, by Proposition 1, the claim follows.

Corollary 2. Every weakly oligomorphic relational structure $\mathbf{A}$ is WKA-equivalent to an endolocal relational structure.

However, for finite relational structures, we can make an even the stronger statement:

Proposition 2. Let $\mathbf{A}=\left(A,\left(\varrho_{A}\right)_{\varrho \in R}\right)$ be a relational structure over a finite set $A$. Then there exists a relational structure $\tilde{\mathbf{A}}=\left(A,\left(\varrho_{\tilde{A}}\right)_{\varrho \in \tilde{R}}\right)$ such that $\tilde{R}$ is finite, $\left[\left(\varrho_{A}\right)_{\varrho \in R}\right]_{\mathrm{WKA}}=$ $\left[\left(\varrho_{A}\right)_{\varrho \in \tilde{R}}\right]_{\mathrm{WKA}}$ and $\tilde{\mathbf{A}}$ is endolocal.

Proof. Suppose that $|A|=n$. Let $\mu \in \Phi_{m}(\exists, \wedge, \vee,=, R)$ for an arbitrary $m \in \mathbb{N}$. Then $\mu^{\mathbf{A}} \in\left[\left(\varrho_{A}\right)_{\varrho \in R}\right]_{\text {WKA }}^{(m)}$.

For each $\bar{a} \in \mu^{\mathbf{A}}$ we define the following two relations:

$$
\begin{array}{r}
\varepsilon_{\bar{a}}:=\left\{(i, j) \mid a_{i}=a_{j}\right\} \\
\sigma_{\mu^{\mathbf{A}, \bar{a}}}:=\left\{\bar{b} \in \mu^{\mathbf{A}} \mid \varepsilon_{\bar{b}} \supseteq \varepsilon_{\bar{a}}\right\}
\end{array}
$$

Then $\sigma_{\mu^{\mathrm{A}}, \bar{a}}=\varphi_{\mu^{\mathrm{A}}, \bar{a}}^{\mathbf{A}}$ for

$$
\varphi_{\mu^{\mathrm{A}}, \bar{a}} \equiv \mu\left(x_{1}, \ldots, x_{m}\right) \wedge\left(\bigwedge_{(i, j) \in \varepsilon_{\bar{a}}}\left(x_{i}=x_{j}\right)\right) .
$$

Therefore, $\sigma_{\mu^{\mathrm{A}}, \bar{a}} \in\left[\left(\varrho_{A}\right)_{\varrho \in R}\right]_{\mathrm{WKA}}^{(m)}$. Moreover,

$$
\mu^{\mathbf{A}}=\bigcup_{\bar{a} \in \mu^{\mathbf{A}}} \sigma_{\mu^{\mathbf{A}}, \bar{a}}=\left(\bigvee_{\bar{a} \in \mu^{\mathbf{A}}} \varphi_{\mu^{\mathbf{A}}, \bar{a}}\right)^{\mathbf{A}}
$$

From this it follows that $\Phi=\left\{\varphi_{\mu^{\mathrm{A}}, \bar{a}} \mid \mu \in \Phi_{m}(\exists, \wedge, \vee,=, R), m \in \mathbb{N}, \bar{a} \in \mu^{\mathbf{A}}\right\}$ is a positive existential elimination set for $\mathbf{A}$. In the following we will transform this positive existential elimination set, to obtain the desired endolocal relational structure.

Take $\varphi_{\mu^{\mathrm{A}}, \bar{a}}$, and consider its corresponding $\sigma_{\mu^{\mathrm{A}}, \bar{a}}$. We take the lexicographically smallest transversal $i_{1}, \ldots, i_{k_{\bar{a}}}$ of $\{1, \ldots, m\} / \varepsilon_{\overline{\varepsilon_{\bar{a}}}}$, and then we define the mapping

$$
\text { rep }:\{1, \ldots, m\} \rightarrow\left\{1, \ldots, k_{\bar{a}}\right\}: i \mapsto j \text {, whenever }[i]_{\varepsilon_{\bar{a}}}=\left[i_{j}\right]_{\varepsilon_{\bar{a}}} \text {. }
$$


Note that $k_{\bar{a}} \leq n$. Using this mapping, we define a new relation

$$
\delta_{\mu^{\mathbf{A}, \bar{a}}}:=\left\{\left(d_{1}, \ldots, d_{k_{\bar{a}}}\right) \mid\left(d_{\operatorname{rep}(1)}, \ldots, d_{\operatorname{rep}(m)}\right) \in \sigma_{\mu^{\mathbf{A}}, \bar{a}}\right\} .
$$

Note that $\delta_{\mu^{\mathbf{A}}, \bar{a}}=\left(\psi_{\mu^{\mathbf{A}}, \bar{a}}\right)^{\mathbf{A}}$, where

$$
\psi_{\mu^{\mathrm{A}}, \bar{a}} \equiv \varphi_{\mu^{\mathrm{A}}, \bar{a}}\left(x_{\mathrm{rep}(1)}, \ldots, x_{\mathrm{rep}(m)}\right),
$$

and, therefore, $\delta_{\mu^{\mathrm{A}}, \bar{a}} \in\left[\left(\varrho_{A}\right)_{\varrho \in R}\right]_{\mathrm{WKA}}^{\left(k_{\bar{a}}\right)}$.

Observe that

$$
\begin{gathered}
\Psi=\left\{\psi_{\mu^{\mathrm{A}}, \bar{a}}\left(x_{i_{1}}, \ldots, x_{i_{\bar{a}}}\right) \mid \mu \in \bigcup_{m \in \mathbb{N}} \Phi_{m}(\exists, \wedge, \vee,=, R), \bar{a} \in \mu^{\mathrm{A}},\right. \\
\left.i_{1}, \ldots, i_{k_{\bar{a}}} \text { is lexicographically smallest transversal of }\{1, \ldots, m\} / \varepsilon_{\bar{a}}\right\}
\end{gathered}
$$

is again a positive existential elimination set for $\mathbf{A}$, because

$$
\left(\varphi_{\mu^{\mathrm{A}}, \bar{a}}\right)^{\mathbf{A}}=\sigma_{\mu^{\mathbf{A}, \bar{a}}}=\left(\psi_{\mu^{\mathrm{A}}, \bar{a}}\left(x_{i_{1}}, \ldots, x_{i_{\bar{a}}}\right) \wedge\left(\bigwedge_{(i, j) \in \varepsilon_{\bar{a}}}\left(x_{i}=x_{j}\right)\right)\right)^{\mathbf{A}} .
$$

Hence,

$$
\mu^{\mathbf{A}}=\left(\bigvee_{\bar{a} \in \mu^{\mathbf{A}}}\left(\psi_{\mu^{\mathbf{A}}, \bar{a}}\left(x_{i_{1}}, \ldots, x_{i_{\bar{a}}}\right) \wedge\left(\bigwedge_{(i, j) \in \varepsilon_{\bar{a}}}\left(x_{i}=x_{j}\right)\right)\right)\right)^{\mathbf{A}} .
$$

Thus, every $\mu^{\mathbf{A}}$ is equivalent in $\mathbf{A}$ to a disjunction of conjunctions of formulae from $\Psi$.

There are finitely many relations of the shape $\delta_{\mu^{\mathrm{A}}, \bar{a}}=\left(\psi_{\mu^{\mathrm{A}}, \bar{a}}\left(x_{i_{1}}, \ldots, x_{i_{\bar{k}}}\right)\right)^{\mathbf{A}}$, because $k_{\bar{a}} \leq n$, for all $\bar{a}$. We define $\tilde{R}:=\left\{\left[\delta_{\mu^{\mathrm{A}}, \bar{a}}\right] \mid \mu \in \Phi_{m}(\exists, \wedge, \vee,=, R), m \in \mathbb{N}, \bar{a} \in \mu^{\mathbf{A}}\right\}$ and $\tilde{\mathbf{A}}:=\left(A,\left(\varrho_{\tilde{A}}\right)_{\varrho \in \tilde{R}}\right)$, where $\left[\delta_{\mu^{\mathrm{A}}, \bar{a}}\right]_{\tilde{\mathbf{A}}}:=\delta_{\mu^{\mathrm{A}}, \bar{a}}=\left(\psi_{\mu^{\mathrm{A}}, \bar{a}}\left(x_{i_{1}}, \ldots, x_{i_{\bar{a}_{\bar{a}}}}\right)\right)^{\mathbf{A}}$.

From what was shown above, it follows that $\mathbf{A}$ and $\tilde{\mathbf{A}}$ are WKA-equivalent.

In order to see that $\Phi^{(0)}(\exists, \wedge, \vee,=, \tilde{R})$ is a positive existential elimination set for $\tilde{\mathbf{A}}$, take any $\tilde{\mu} \in \Phi^{(m)}(\exists, \wedge, \vee,=, \tilde{R})$ ( $m$ arbitrary). Then there exists a $\mu \in$ $\Phi^{(m)}(\exists, \wedge, \vee,=, R)$ with $\mu^{\mathbf{A}}=\tilde{\mu}^{\tilde{\mathbf{A}}}$. However, from (1), and from the definition of $\tilde{\mathbf{A}}$ it follows that

$$
\tilde{\mu}^{\tilde{\mathbf{A}}}=\left(\bigvee_{\bar{a} \in \mu^{\mathrm{A}}}\left(\left[\delta_{\mu^{\mathrm{A}}, \bar{a}}\right]\left(x_{i_{1}}, \ldots, x_{i_{\overline{k_{\bar{a}}}}}\right) \wedge\left(\bigwedge_{(i, j) \in \varepsilon_{\bar{a}}}\left(x_{i}=x_{j}\right)\right)\right)\right)^{\tilde{\mathbf{A}}} .
$$

From the Main Theorem, it follows that $\tilde{\mathbf{A}}$ is endolocal. 
2.5.5. Weakly oligomorphic weak Krasner algebras. As we already pointed out, the property of relational structures to be weakly oligomorphic is stable under WKA-equivalence. Hence, this is more a property of weak Krasner algebras than of relational structures. This motivates the following definition:

Definition. Let $W$ be a weak Krasner algebra. Then we say that $W$ is weakly oligomorphic if for every $m \in \mathbb{N}$ the $m$-ary part $W^{(m)}$ contains only finitely many relations.

Proposition 1. Let W be a weakly oligomorphic weak Krasner algebra over a countable basic set. Then $W$ is locally closed, i.e.

$$
W=\operatorname{Inv} \text { End } W .
$$

Proof. Let $\mathbf{A}=\left(A,\left(\varrho_{\mathbf{A}}\right)_{\varrho \in R}\right)$ a relational structure with the property $\left[\left(\varrho_{\mathbf{A}}\right)_{\varrho \in R}\right]_{W K A}=$ $W$. Since $W$ is weakly oligomorphic, it follows that the context $\mathbb{K}_{m}(\mathbf{A})$ has finitely many extents, because every extent is an intersection of elements from $\left[\left(\varrho_{\mathrm{A}}\right)_{\varrho \in R}\right]_{\mathrm{WKA}}^{(m)}$. Hence, $\mathbf{A}$ is weakly oligomorphic. By 2.5 .4 (Corollary 2), it follows that $\mathbf{A}$ is WKA-equivalent to an endolocal and weakly oligomorphic relational structure $\tilde{\mathbf{A}}$. From the Main Theorem and from the fact that $\mathbf{A}$ is countable, it follows that $\tilde{\mathbf{A}}$ is homomorphism-homogeneous. Finally, from 2.5 .2 we obtain that

$$
\text { Inv End } W=\operatorname{Inv} \text { End } \tilde{\mathbf{A}}=W \text {. }
$$



.... when you have eliminated the impossible, whatever remains, however improbable, must be the truth.

\section{Chapter 3.}

(Sir Arthur Conan Doyle)

\section{Homomorphism-homogeneous relational structures}

This chapter is dedicated to a systematic study of homomorphism-homogeneous relational structures. Using the results collected in the Main Theorem, in 3.1 we derive a criterion for characterizing relational structures of this kind. This criterion is called the minimal witness criterion and it is forming the basis of a method for the elimination of all non homomorphism-homogeneous relational structures from a given class of relational structures. In 3.2 we turn our attention to binary homomorphism-homogeneous structures and we use the minimal witness criterion in order to characterize all transitive relations that have the one-point extension property and this, by the Main Theorem, gives us a characterization of homomorphism-homogeneous transitive relations on countable basic sets. In the sequel we treat tolerance relations. In particular, we describe a strategy for the constructive enumeration of homomorphism-homogeneous tolerance relations, and we find a number of infinite families of such relations.

\subsection{Minimal witness criterion}

Let us continue our efforts to find a useful criterion for a relational structure not to have the one-point extension property.

3.1.1. Witness. From the Main Theorem we know that every homomorphismhomogeneous relational structure has the weak one-point extension property, and that every relational structure on a countable basic set with the weak onepoint extension property is homomorphism-homogeneous. However, if a relational structure does not posses some property, then there has to exist a counterargument, and a counterargument for the weak one-point extension property will be the existence of what we will call a witness.

More formally, a witness is a quadruple $\left(\mathbf{B}_{1}, \mathbf{B}_{2}, f, c\right)$, such that $\mathbf{B}_{1}$ is a finite substructure of $\mathbf{A}, c$ is a weak center of $\mathbf{B}_{1}$ in $A, \mathbf{B}_{2}$ is a substructure of $\mathbf{A}$, and $f: \mathbf{B}_{1} \rightarrow \mathbf{B}_{\mathbf{2}}$ is surjective, but $f$ cannot be extended to $\mathbf{B}_{\mathbf{1}} \cup\{c\}$.

Our next aim is to define a quasi-order on witnesses. For this purpose, we need to introduce two auxiliary relations - a homomorphic image and a subwitness, that will be denoted by $\rightarrow$ and $\leq$, respectively.

We say that a witness $\left(\mathbf{B}_{3}, \mathbf{B}_{4}, g, d\right)$ is a homomorphic image of a witness $\left(\mathbf{B}_{1}, \mathbf{B}_{2}, f, c\right)$, and write $\left(\mathbf{B}_{1}, \mathbf{B}_{2}, f, c\right) \rightarrow\left(\mathbf{B}_{3}, \mathbf{B}_{4}, g, d\right)$, if there exist epimorphisms 
$h_{1}, h_{2}$ and $h_{3}$, such that the following diagram commutes:

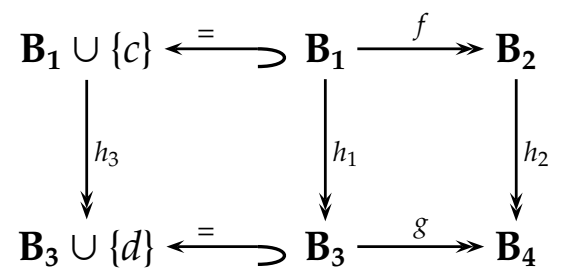

We say that a witness $\left(\mathbf{B}_{1}, \mathbf{B}_{2}, f, c\right)$ is a subwitness of a witness $\left(\mathbf{B}_{3}, \mathbf{B}_{4}, g, c\right)$, and write $\left(\mathbf{B}_{1}, \mathbf{B}_{2}, f, c\right) \leq\left(\mathbf{B}_{3}, \mathbf{B}_{4}, g, c\right)$, if $\mathbf{B}_{1}$ is a substructure of $\mathbf{B}_{3}, \mathbf{B}_{2}$ is a substructure of $\mathbf{B}_{4}$ and the following diagram commutes:

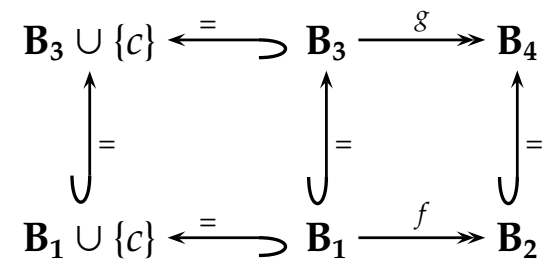

Once these two relations on witnesses are established, we are ready to introduce a quasi-order on witnesses.

3.1.2. Quasi-order on witnesses. We say that a witness $\left(\mathbf{B}_{1}, \mathbf{B}_{2}, f, c\right)$ is below a witness $\left(\mathbf{B}_{3}, \mathbf{B}_{4}, g, d\right)$, and write $\left(\mathbf{B}_{1}, \mathbf{B}_{2}, f, c\right) \leq\left(\mathbf{B}_{3}, \mathbf{B}_{4}, g, d\right)$ if there exist a witness $\left(\mathbf{B}_{5}, \mathbf{B}_{6}, h, d\right)$ such that the following holds:

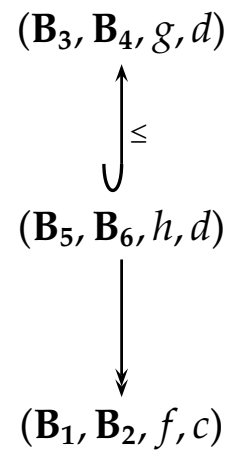

From the definition of the relation "below", it follows immediately that it is reflexive, so if we succeed to show that it is also transitive, "below" is going to be a quasi-order we were looking for.

So, let $\left(\mathbf{B}_{1}, \mathbf{D}_{1}, f_{1}, c_{1}\right) \leq\left(\mathbf{B}_{2}, \mathbf{D}_{2}, f_{2}, c_{2}\right)$ and $\left(\mathbf{B}_{2}, \mathbf{D}_{2}, f_{2}, c_{2}\right) \leq\left(\mathbf{B}_{3}, \mathbf{D}_{3}, f_{3}, c_{3}\right)$. Then there exist witnesses $\left(\mathbf{B}_{4}, \mathbf{D}_{4}, f_{4}, c_{2}\right)$ and $\left(\mathbf{B}_{5}, \mathbf{D}_{5}, f_{5}, c_{3}\right)$ such that the following 
diagram commutes:

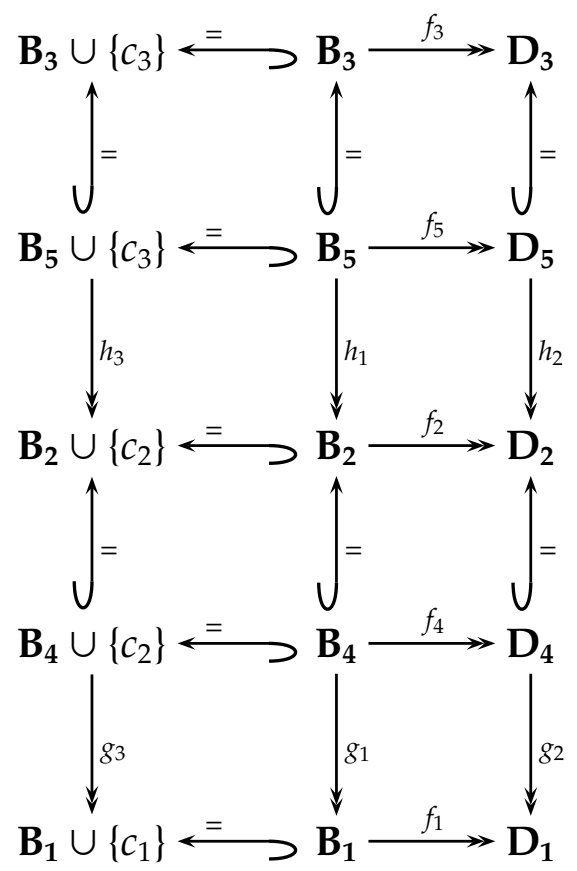

We define now $\overline{f_{5}}: h_{1}^{-1}\left(\mathbf{B}_{4}\right) \rightarrow h_{2}^{-1}\left(\mathbf{D}_{4}\right)$ by $\bar{f}_{5}(x)=f_{5}(x)$, and claim:

1. $\left(h_{1}^{-1}\left(\mathbf{B}_{4}\right), h_{2}^{-1}\left(\mathbf{D}_{4}\right), \overline{f_{5}}, c_{3}\right) \leq\left(\mathbf{B}_{3}, \mathbf{D}_{3}, f_{3}, c_{3}\right)$, and

2. $\left(h_{1}^{-1}\left(\mathbf{B}_{4}\right), h_{2}^{-1}\left(\mathbf{D}_{4}\right), \bar{f}_{5}, c_{3}\right) \rightarrow\left(\mathbf{B}_{1}, \mathbf{D}_{1}, f_{1}, c_{1}\right)$.

Observe now the following diagram:

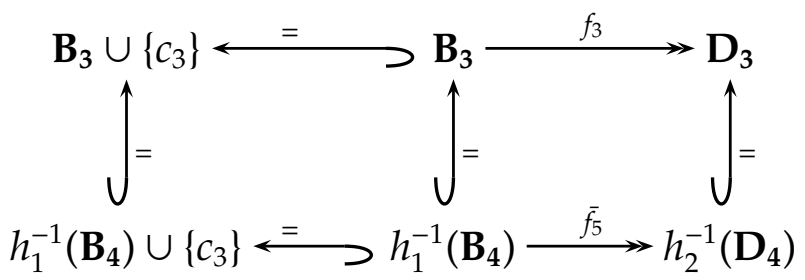

Its left hand side is obviously commuting. For the right hand side, take arbitrary $x \in h_{1}^{-1}\left(B_{4}\right)$. Then

$$
\bar{f}_{5}(x)=f_{5}(x)=f_{3}(x),
$$

so the above given diagram is commuting, so $\left(h_{1}^{-1}\left(\mathbf{B}_{4}\right), h_{2}^{-1}\left(\mathbf{D}_{4}\right), \overline{f_{5}}, c_{3}\right)$ is a subwitness of $\left(\mathbf{B}_{3}, \mathbf{D}_{3}, f_{3}, c_{3}\right)$.

For the proof of the second claim, it suffices to show that this diagram commutes:

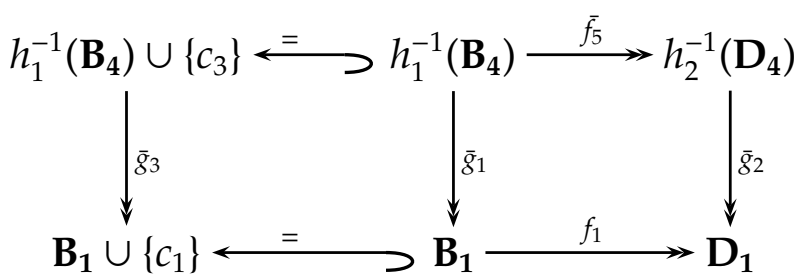


where

$$
\begin{array}{lll}
\bar{g}_{1}(x)=g_{1}\left(h_{1}(x)\right) & \text { for } \quad x \in h_{1}^{-1}\left(B_{4}\right), \\
\bar{g}_{2}(x)=g_{2}\left(h_{2}(x)\right) & \text { for } \quad x \in h_{2}^{-1}\left(D_{4}\right), \\
\bar{g}_{3}(x)=g_{3}\left(h_{3}(x)\right) & \text { for } \quad x \in h_{1}^{-1}\left(B_{4}\right) \cup\left\{c_{3}\right\} .
\end{array}
$$

Take $x \in h_{1}^{-1}\left(B_{4}\right)$. Then

$$
\begin{aligned}
\bar{g}_{2}\left(\bar{f}_{5}(x)\right) & =\bar{g}_{2}\left(f_{5}(x)\right)=g_{2}\left(h_{2}\left(f_{5}(x)\right)\right)=g_{2}\left(f_{2}\left(h_{1}(x)\right)\right) \\
& =g_{2}\left(f_{4}\left(h_{1}(x)\right)\right)=f_{1}\left(g_{1}\left(h_{1}(x)\right)\right)=f_{1}\left(\bar{g}_{1}(x)\right),
\end{aligned}
$$

and the right hand side of the diagram commutes. For the left hand side, one has to show that $\bar{g}_{3}\left(c_{3}\right)=c_{1}$ and for every $x \in h_{1}^{-1}\left(B_{4}\right)$, we have that $\bar{g}_{3}(x)=\bar{g}_{1}(x)$.

Clearly, $\bar{g}_{3}\left(c_{3}\right)=g_{3}\left(h_{3}\left(c_{3}\right)\right)=g_{3}\left(c_{2}\right)=c_{1}$. For $x \in h_{1}^{-1}\left(B_{4}\right)$, we obtain

$$
\bar{g}_{3}(x)=g_{3}\left(h_{3}(x)\right)=g_{1}\left(h_{1}(x)\right)=\bar{g}_{1}(x) .
$$

Since both claims hold, we conclude that $\left(\mathbf{B}_{1}, \mathbf{D}_{1}, f_{1}, c_{1}\right) \leq\left(\mathbf{B}_{3}, \mathbf{D}_{3}, f_{3}, c_{3}\right)$, so relation "below" is transitive, and it is a quasi-order on witnesses.

3.1.3. Isomorphic witnesses. We say that a witness $\left(\mathbf{B}_{1}, \mathbf{B}_{2}, f, c\right)$ is isomorphic to a witness $\left(\mathbf{B}_{3}, \mathbf{B}_{4}, g, d\right)$, and write $\left(\mathbf{B}_{1}, \mathbf{B}_{2}, f, c\right) \cong\left(\mathbf{B}_{3}, \mathbf{B}_{4}, g, d\right)$ if there exist isomorphisms $\varphi_{1}, \varphi_{2}$ and $\varphi_{3}$ such that the following diagram commutes:

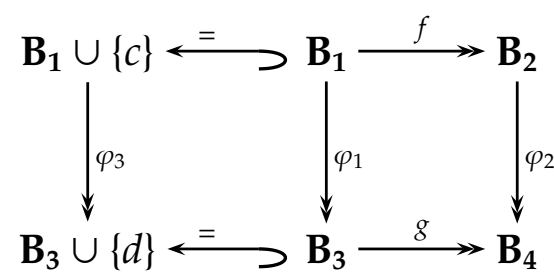

Proposition 1. Let $\left(\mathbf{B}_{1}, \mathbf{B}_{2}, f, c\right)$ and $\left(\mathbf{B}_{3}, \mathbf{B}_{4}, g, d\right)$ be witnesses. If $\left(\mathbf{B}_{1}, \mathbf{B}_{2}, f, c\right) \leq$ $\left(\mathbf{B}_{3}, \mathbf{B}_{4}, g, d\right)$ and $\left(\mathbf{B}_{3}, \mathbf{B}_{4}, g, d\right) \leq\left(\mathbf{B}_{1}, \mathbf{B}_{2}, f, c\right)$, then $\left(\mathbf{B}_{1}, \mathbf{B}_{2}, f, c\right) \cong\left(\mathbf{B}_{3}, \mathbf{B}_{4}, g, d\right)$. 
Proof. From the given conditions it follows that this diagram commutes:

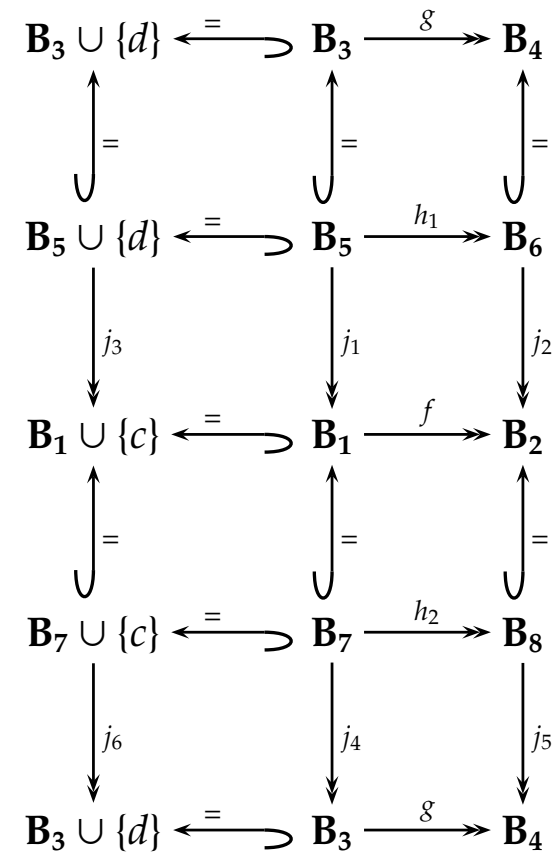

Note that from this diagram it follows that

$$
\begin{aligned}
& \left|B_{3}\right| \leq\left|B_{7}\right| \leq\left|B_{1}\right| \leq\left|B_{5}\right| \leq\left|B_{3}\right| \quad \text { and } \\
& \left|B_{4}\right| \leq\left|B_{8}\right| \leq\left|B_{2}\right| \leq\left|B_{6}\right| \leq\left|B_{4}\right|
\end{aligned}
$$

so

$$
\begin{aligned}
& \left|B_{3}\right|=\left|B_{7}\right|=\left|B_{1}\right|=\left|B_{5}\right| \text { and } \\
& \left|B_{4}\right|=\left|B_{8}\right|=\left|B_{2}\right|=\left|B_{6}\right| .
\end{aligned}
$$

Hence, $B_{5}=B_{3}, B_{7}=B_{1}, B_{6}=B_{4}$ and $B_{8}=B_{2}$, so this allows us to simplify the given diagram to

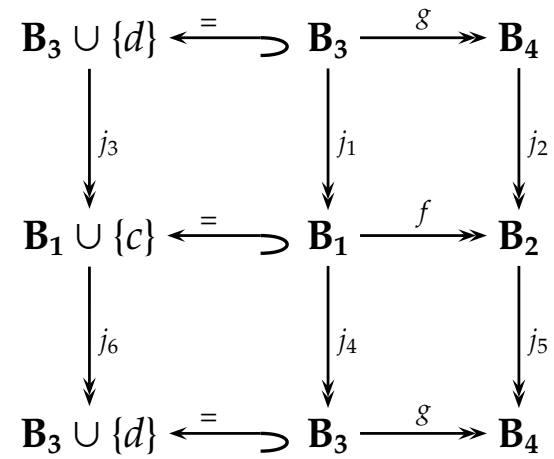

where all $j_{i}$ 's are both bijections and homomorphisms. We will show that $j_{1}, j_{2}$ and $j_{3}$ are isomorphism. It suffices to show that $j_{1}$ is an embedding (for other two mappings it can be proved analogously).

As we have already seen, $j_{1}$ is a bijective homomorphism, so $\left|B_{3}\right|=\left|B_{1}\right|$, and for every $\varrho \in R,\left|\varrho_{\mathbf{B}_{3}}\right| \leq\left|\varrho_{\mathbf{B}_{1}}\right|$. Since $j_{4}$ is also a bijective homomorphism, we obtain 
that $\left|B_{1}\right|=\left|B_{3}\right|$, and for every $\varrho \in R,\left|\varrho_{\mathbf{B}_{1}}\right| \leq\left|\varrho_{\mathbf{B}_{3}}\right|$. Hence, $\left|B_{1}\right|=\left|B_{3}\right|$ and for every $\varrho \in R,\left|\varrho_{\mathbf{B}_{1}}\right|=\left|\varrho_{\mathbf{B}_{3}}\right|$, so $J_{1}$ is an embedding, and moreover, it is an isomorphism.

Thus, $\left(\mathbf{B}_{1}, \mathbf{B}_{2}, f, c\right) \cong\left(\mathbf{B}_{3}, \mathbf{B}_{4}, g, d\right)$.

3.1.4. Minimal witness criterion. We say that $\left(\mathbf{B}_{1}, \mathbf{B}_{2}, f, c\right)$ is a minimal witness if it is a minimal element in the quasi-order of witnesses, i.e. for any other witness $\left(\mathbf{B}_{3}, \mathbf{B}_{4}, g, d\right)$ holds that whenever $\left(\mathbf{B}_{3}, \mathbf{B}_{4}, g, d\right) \leq\left(\mathbf{B}_{1}, \mathbf{B}_{2}, f, c\right)$, then $\left(\mathbf{B}_{1}, \mathbf{B}_{2}, f, c\right) \leq$ $\left(\mathbf{B}_{3}, \mathbf{B}_{4}, g, d\right)$.

\section{Minimal witness criterion:}

A structure A has weak one-point extension property

if and only if it has no minimal witnesses.

Proposition 1. Let $\mathbf{A}=\left(A,\left(\varrho_{\mathbf{A}}\right)_{\varrho \in R}\right)$ be such that exactly one relation in $R$ is binary and symmetric, and all others are unary. If $\left(\mathbf{B}_{1}, \mathbf{B}_{2}, f, c\right)$ is a minimal witness, then $f$ is bijective.

Proof. Let $\left(\mathbf{B}_{1}, \mathbf{B}_{2}, f, c\right)$ be a minimal witness, and suppose that $f$ is not bijective. Let $B_{3}$ be a transversal of $B_{1} / \operatorname{ker} f$, let $B_{4}:=B_{2}$ and let $g:=f \uparrow_{B_{3}}$.

Note that then $\mathbf{B}_{3}<\mathbf{B}_{1}$. In the next step, we prove that $\left(\mathbf{B}_{3}, \mathbf{B}_{4}, g, c\right)$ is a witness. For this we have to show that

- $c$ is a weak center of $\mathbf{B}_{3}$, and

- $g$ cannot be extended to $\mathbf{B}_{3} \cup\{c\}$.

Since $c$ is a weak center of $\mathbf{B}_{1}$, we have that for every $b \in B_{1}$ there exists a $\varrho \in R$ (which is in this case precisely the unique binary relation in $R$ ), and a $\pi \in \operatorname{Sym}\{1,2\}$ such that $(c, b)^{\pi} \in \varrho_{\mathbf{A}}$. Take any $b \in B_{3}$. Then $b \in B_{1}$, and $c$ is a weak center of $\mathbf{B}_{3}$ by definition.

Suppose now that $g$ can be extended to $\mathbf{B}_{3} \cup\{c\}$. Then there exists a $d$ such that

$$
\tilde{g}(x)= \begin{cases}g(x), & \text { if } x \in B_{3} \\ d, & \text { if } x=c .\end{cases}
$$

is an extension of $g$ to $\mathbf{B}_{3} \cup\{c\}$. We claim that then

$$
\tilde{f}(x)= \begin{cases}f(x), & \text { if } x \in B_{1} \\ d, & \text { if } x=c .\end{cases}
$$

is an extension of $f$ to $\mathbf{B}_{1} \cup\{c\}$. In order to prove this claim, we distinguish two cases: $\varrho$ is unary and $\varrho$ is binary.

If $\varrho$ is unary, then if $c \in \varrho_{\mathbf{A}}$, then $\tilde{g}(c) \in \varrho_{\mathbf{A}}$, so $d \in \varrho_{\mathbf{A}}$, and $\tilde{f}(c)=d \in \varrho_{\mathbf{A}}$. However, if $\varrho$ is binary, then take $(c, x) \in \varrho_{\mathbf{A}}$, where $x \in B_{1} \cup\{c\}$. If $x \in B_{3} \cup\{c\}$, then $(\tilde{f}(c), \tilde{f}(x))=(\tilde{g}(c), \tilde{g}(x)) \in \varrho_{\mathbf{A}}$. We consider now the case $x \in B_{1} \backslash B_{3}$. Then there exists exactly one $x^{\prime} \in B_{3}$ such that $f\left(x^{\prime}\right)=f(x)$, so

$$
(\tilde{f}(c), \tilde{f}(x))=(\tilde{g}(c), f(x))=\left(\tilde{g}(c), f\left(x^{\prime}\right)\right)=\left(\tilde{g}(c), g\left(x^{\prime}\right)\right)=\left(\tilde{g}(c), \tilde{g}\left(x^{\prime}\right)\right) .
$$


Since $c$ is a weak center of $\mathbf{B}_{3}$ and $\varrho$ is symmetric, we have $\left(c, x^{\prime}\right) \in \varrho_{\mathbf{A}}$ and $\left(x^{\prime}, c\right) \in \varrho_{\mathbf{A}}$. Further we obtain $\left(\tilde{g}(c), \tilde{g}\left(x^{\prime}\right)\right) \in \varrho_{\mathbf{A}}$, so $(\tilde{f}(c), \tilde{f}(x)) \in \varrho_{\mathbf{A}}$. Hence, $\tilde{f}$ is an extension of $f$ and we arrive to a contradiction.

Therefore, $\left(\mathbf{B}_{3}, \mathbf{B}_{4}, g, c\right)$ is a witness. Moreover, the diagram

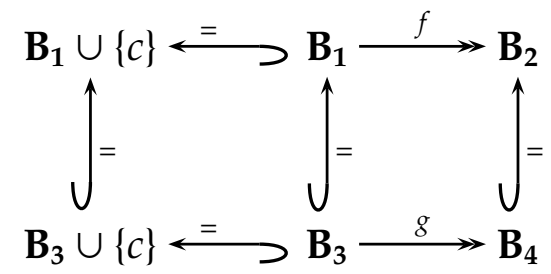

commutes, so having in mind that $\mathbf{B}_{3}<\mathbf{B}_{1}$, we conclude that $\left(\mathbf{B}_{3}, \mathbf{B}_{4}, g, c\right)$ is a proper subwitness of $\left(\mathbf{B}_{1}, \mathbf{B}_{2}, f, c\right)$, and $\left(\mathbf{B}_{1}, \mathbf{B}_{2}, f, c\right)$ cannot be a minimal one, which is a contradiction.

Lemma. Let $\mathbf{A}=\left(A,\left(\varrho_{\mathbf{A}}\right)_{\varrho \in R}\right)$ be such that $R$ consists of exactly one binary antisymmetric transitive relation $\sigma$. If $\left(\mathbf{B}_{1}, \mathbf{B}_{2}, f, c\right)$ is a witness, then for every equivalence class $S$ of ker $f$ either $\forall x \in S: \quad(c, x) \in \sigma$ or $\forall x \in S: \quad(x, c) \in \sigma_{\mathbf{A}}$.

Proof. We will prove the claim indirectly. Suppose that there exists an equivalence class $S$ of $\operatorname{ker} f$ such that there are $y, z \in S$, where $(y, c) \in \sigma_{\mathbf{A}}$ and $(c, z) \in \sigma_{\mathbf{A}}$.

Case 1. $S$ is the unique equivalence class of that kind. From $(y, c) \in \sigma_{\mathbf{A}}$ and $(c, z) \in \sigma_{\mathbf{A}}$, it follows that $(y, z) \in \sigma_{\mathbf{A}}$, so $(f(y), f(z)) \in \sigma_{\mathbf{A}}$. We define

$$
\tilde{f}(x)= \begin{cases}f(x), & \text { if } x \in B_{1} \\ f(y), & \text { if } x=c\end{cases}
$$

and we show that $\tilde{f}$ is an extension of $f$ to $\mathbf{B}_{\mathbf{1}} \cup\{c\}$. Take $(c, x) \in \sigma_{\mathbf{A}}$. Then

$$
(\tilde{f}(x), \tilde{f}(y))= \begin{cases}(f(y), f(x)), & \text { if } x \in B_{1} \\ (f(y), f(y)), & \text { if } x=c .\end{cases}
$$

If $x=c$, then from $(y, z) \in \operatorname{ker} f \cap \sigma_{\mathbf{A}}$ it follows that $(f(y), f(z)) \in \sigma_{\mathbf{A}}$ and $f(y)=f(z)$, so $(f(y), f(y)) \in \sigma_{\mathbf{A}}$. On the other side, if $x \in B_{1}$, then from $(c, x) \in \sigma_{\mathbf{A}}$ and $(y, c) \in \sigma_{\mathbf{A}}$, we obtain $(y, x) \in \sigma_{\mathbf{A}}$, so $(f(y), f(x)) \in \sigma_{\mathbf{A}}$. For $(x, c) \in \sigma_{\mathbf{A}}$ one can prove it analogously.

Hence, $\tilde{f}$ is indeed an extension, so $\left(\mathbf{B}_{1}, \mathbf{B}_{2}, f, c\right)$ is not a witness, which is a contradiction.

Case 2. There exists at least one more equivalence class $T$ of $\operatorname{ker} f$ of the given kind. Then there exist $u, v \in T$ such that $(u, c) \in \sigma_{\mathbf{A}}$ and $(c, v) \in \sigma_{\mathbf{A}}$. From $(u, c) \in \sigma_{\mathbf{A}}$ and $(c, z) \in \sigma_{\mathbf{A}}$, it follows that $(u, z) \in \sigma_{\mathbf{A}}$, so $(f(u), f(z)) \in \sigma_{\mathbf{A}}$. Also, from $(y, c) \in \sigma_{\mathbf{A}}$ and $(c, v) \in \sigma_{\mathbf{A}}$, we obtain $(y, v) \in \sigma_{\mathbf{A}}$, so $(f(y), f(v)) \in \sigma_{\mathbf{A}}$. On the other hand, $(f(z), f(u))=(f(y), f(v)) \in \sigma_{\mathbf{A}}$, so by antisymmetry of $\mathbf{A}$, we obtain $f(z)=f(u)$, which is a contradiction, since $S \neq T$.

Proposition 2. Let $\mathbf{A}=\left(A,\left(\varrho_{\mathbf{A}}\right)_{\varrho \in R}\right)$ be such that $R$ consists of exactly one binary antisymmetric transitive relation $\sigma$. If $\left(\mathbf{B}_{1}, \mathbf{B}_{2}, f, c\right)$ is a minimal witness, then $f$ is bijective. 
Proof. Suppose that $f$ is not bijective and let $B_{3}$ be a transversal of $B_{1} / \operatorname{ker} f$. Moreover, define $B_{4}:=B_{2}$, and $g:=f \uparrow_{B_{3}}$. Our goal is to show that $\left(\mathbf{B}_{3}, \mathbf{B}_{4}, g, c\right)$ is a witness. It is easy to see that $c$ is a weak center of $\mathbf{B}_{3}$, so it remains to show that $g$ cannot be extended to $\mathbf{B}_{\mathbf{3}} \cup\{c\}$.

Suppose the opposite, i.e. that there exists a $d \in A$ such that

$$
\tilde{g}(x)= \begin{cases}g(x), & \text { if } x \in B_{3} \\ d, & \text { if } x=c .\end{cases}
$$

is an extension of $g$ to $\mathbf{B}_{\mathbf{3}} \cup\{c\}$. We claim that

$$
\tilde{f}(x)= \begin{cases}f(x), & \text { if } x \in B_{1} \\ d, & \text { if } x=c\end{cases}
$$

is then an extension of $f$ to $\mathbf{B}_{\mathbf{1}} \cup\{c\}$. Take $(c, x) \in \sigma_{\mathbf{A}}$, where $x \in \mathbf{B}_{\mathbf{1}} \cup\{c\}$ (for $(x, c) \in \sigma_{\mathbf{A}}$ one can prove it analogously). Then

$$
(\tilde{f}(c), \tilde{f}(y))= \begin{cases}(d, f(x)), & \text { if } x \in B_{1} \\ (d, d)), & \text { if } x=c .\end{cases}
$$

If $x=c$, then from $(c, c) \in \sigma_{\mathbf{A}}$, it follows $(d, d)=(\tilde{g}(c), \tilde{g}(c)) \in \sigma_{\mathbf{A}}$. On the other hand, if $x \in B_{1}$, then there exists a unique $x^{\prime} \in B_{3}$, such that $\left(x, x^{\prime}\right) \in \operatorname{ker} f$. Then from the previous Lemma, it follows that $\left(c, x^{\prime}\right) \in \sigma_{\mathbf{A}}$, so $\left(d, g\left(x^{\prime}\right)\right)=(\tilde{g}(c), \tilde{g}(x)) \in \sigma_{\mathbf{A}}$, i.e. $(d, f(x))=\left(d, f\left(x^{\prime}\right)\right)=\left(d, g\left(x^{\prime}\right)\right) \in \sigma_{\mathbf{A}}$. Hence, $\left(\mathbf{B}_{1}, \mathbf{B}_{2}, f, c\right)$ is not a witness, which is a contradiction.

It follows that $\left(\mathbf{B}_{3}, \mathbf{B}_{4}, g, c\right)$ is a witness, and moreover, it is a proper subwitness of $\left(\mathbf{B}_{1}, \mathbf{B}_{2}, f, c\right)$, so $\left(\mathbf{B}_{1}, \mathbf{B}_{2}, f, c\right)$ cannot be minimal - a contradiction.

Thus, $f$ is bijective.

\subsection{Binary homomorphism-homogeneous relations}

In this section we turn our attention to the application of the minimal witness criterion to the binary relations.

3.2.1. Minimal witnesses for antisymmetric transitive relations. Let there be given a relational structure $\mathbf{A}=(A, \varrho)$ such that $\varrho$ is an antisymmetric transitive relation. Our goal is to describe possible minimal witnesses for such relational structures. By 3.1.4(Proposition 2) we obtain that for every minimal witness $\left(\mathbf{B}_{1}, \mathbf{B}_{2}, f, c\right)$ of $\mathbf{A}$ holds that $f$ is bijective. 
Let, further, $\left(\mathbf{B}_{1}, \mathbf{B}_{2}, f, c\right)$ be an arbitrary minimal witness in $\mathbf{A}$. Since $c$ is a weak center of $\mathbf{B}_{\mathbf{1}}$, it follows that for every $b \in B_{1}$ either $(b, c) \in \varrho$ or $(c, b) \in \varrho$, so we can split $B_{1}$ into a disjoint union $B_{11} \dot{\cup} B_{12}$, given by

$$
\begin{aligned}
& B_{11}:=\left\{b \in B_{1} \mid(b, c) \in \varrho\right\} \\
& B_{12}:=\left\{b \in B_{1} \mid(c, b) \in \varrho\right\} .
\end{aligned}
$$

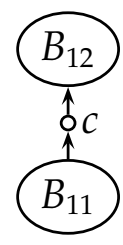

Moreover, $f$ induces a splitting of $B_{2}$ into corresponding parts $B_{21}:=$ $f\left(B_{11}\right)$ and $B_{22}:=f\left(B_{12}\right)$, and we know that there is no element $d$ such that

$$
\forall b \in B_{21}:(b, d) \in \varrho \text { and } \forall b^{\prime} \in B_{22}:\left(d, b^{\prime}\right) \in \varrho .
$$

In order to make the analysis that follows reader friendly, we will distinguish two kinds of elements in A: for $x \in A$ with a property that $(x, x) \in \varrho$ we will say that $x$ has a loop in $\varrho$ and we will draw it as $\mathbf{\Delta}$; otherwise, we will say that $x$ is loopless in $\varrho$ and present it by $\Delta$. However, if the nature of the element in question is not important, we will simply draw o. Additionally, for $B \subseteq A$, we will call a $b \in B$ a sink for $B$ if there is no $a \in B \backslash\{b\}$ such that $(b, a) \in \varrho$. We will also use the notion of independent set. For a $B \subseteq A$ we say that it is independent in $\mathbf{A}$ if for every two distinct elements $b_{1}, b_{2} \in B$ neither $\left(b_{1}, b_{2}\right) \in \varrho$ nor $\left(b_{2}, b_{1}\right) \in \varrho$.

Claim 1. $B_{11}$ and $B_{12}$ are independent sets.

We will first show that the claim holds for $B_{11}$. Suppose that $B_{11}$ is not an independent set. Then the set $B_{11}^{M}$ of all sinks of $\mathbf{B}_{11}$ is a proper subset of $B_{11}$. Define $\tilde{B}_{1}:=B_{11}^{M} \cup B_{12}, \tilde{B}_{2}:=f\left(\tilde{B}_{1}\right)$, and $\tilde{f}:=f \uparrow_{\tilde{B}_{1}}$. We claim that $\left(\tilde{\mathbf{B}}_{1}, \tilde{\mathbf{B}}_{2}, \tilde{f}, c\right)$ is again a witness, and moreover, a proper subwitness of $\left(\mathbf{B}_{1}, \mathbf{B}_{2}, f, c\right)$.

It is clear that $c$ is a weak center of $\tilde{\mathbf{B}}_{1}$. It is left to show that $\tilde{f}$ cannot be extended to $\tilde{\mathbf{B}}_{1}$. Suppose the opposite, i.e. that there exists a $d \in A$ such that

$$
\hat{f}(x)= \begin{cases}\tilde{f}(x), & \text { if } x \in \tilde{B}_{1} \\ d, & \text { if } x=c\end{cases}
$$

is an extension of $\tilde{f}$. Note that $(c, d)$ is then not of the shape $(\boldsymbol{\Lambda}, \Delta)$. Further, for $b \in B_{11}^{M}$ we obtain $(f(b), d)=(\hat{f}(b), \hat{f}(c)) \in \varrho$. On the other hand, for $b \in B_{12}$ holds $(c, b) \in \varrho$, so $(d, f(b))=(\hat{f}(c), \hat{f}(b)) \in \varrho$. Moreover, for every $b \in B_{11} \backslash B_{11}^{M}$, there is a $b^{M} \in B_{11}^{M}$ such that $\left(b, b^{M}\right) \in \varrho$, so $\left(f(b), f\left(b^{M}\right)\right) \in \varrho$, and by transitivity of $\varrho$, it follows that $(f(b), d) \in \varrho$. Hence, there is a $d \in A$ such that $f$ can be extended, implying that $\left(\mathbf{B}_{1}, \mathbf{B}_{2}, f, c\right)$ is not a witness, which is a contradiction. Hence, $B_{11}$ is an independent set and one can prove analogously that $B_{12}$ is also an independent set.

Claim 2. $B_{11}$ and $B_{12}$ cannot be empty sets at the same time. If $B_{1 i}, i=1,2$, is a singleton, then its single element is loopless. Otherwise, $B_{1 i}, i=1,2$, has at least two elements. 


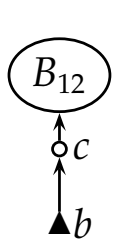

If $B_{11}$ and $B_{12}$ are empty at the same time, then $\left(\mathbf{B}_{1}, \mathbf{B}_{2}, f, c\right)$ is not a witness. Without loss of generality, suppose that $B_{11}$ is not empty, and suppose, contrary to our claim, that $B_{11}$ has precisely one element $b$ with a loop. We claim that $f$ can be then extended to

$$
\hat{f}(x)= \begin{cases}f(x), & \text { if } x \in B_{1} \\ f(b), & \text { if } x=c\end{cases}
$$

Clearly, $\hat{f}$ is a homomorphism, and $\left(\mathbf{B}_{1}, \mathbf{B}_{2}, f, c\right)$ is not a witness, which is a contradiction.

Claim 3. $B_{21}$ and $B_{22}$ are independent sets.

Suppose that $B_{21}$ is not an independent set. Then the set $B_{21}^{M}$ of all sinks of $B_{21}$ is a proper subset of $B_{21}$. Define $\tilde{B}_{2}:=B_{21}^{M} \cup B_{22}, \tilde{B}_{1}:=f^{-1}\left(\tilde{B}_{2}\right)$, and $\tilde{f}:=f \uparrow_{\tilde{B}_{1}}$. It is easy to show that $\left(\tilde{\mathbf{B}}_{1}, \tilde{\mathbf{B}}_{2}, \tilde{f}, c\right)$ is again a witness, and moreover, a proper subwitness of $\left(\mathbf{B}_{1}, \mathbf{B}_{2}, f, c\right)$, which leads us to a contradiction as in the proof of the Claim 1.

From Claims 2 and 3 it follows directly

Claim 4. $B_{21}$ and $B_{22}$ cannot be empty sets at the same time. If $B_{2 i}, i=1,2$, is a singleton, then its single element is loopless. Otherwise, $B_{2 i}, i=1,2$, has at least two elements.

Note that if the single element has a loop, then the mapping that maps the whole $B_{1}$ onto it is a homomorphism that extends $f$.

Claim 5. If $B_{2 i}, i=1,2$ is not empty, then

- either it is a loopless singleton,

- or it has precisely two elements,

- or it consists of $k$ elements with a loop, $k \geq 3$, and $c$ is loopless.

For the proof of the claim we distinguish two cases, depending on the nature of $c$. It is enough prove it for $B_{21}$, since the proof for $B_{22}$ is analogous.

Case 1. Let $c$ have a loop. We will show that if $B_{21}$ has at least two elements, then it has precisely two elements. Suppose that $B_{21}$ has at least three elements. We choose an element $b_{1}$ in the following manner:

- if $B_{21}$ contains an element that has a loop and its preimage under $f$ also has a loop, then let $b_{1}$ be this element;

- if non of the elements of $B_{11}$ has a loop and $B_{21}$ contains an element that has a loop, then
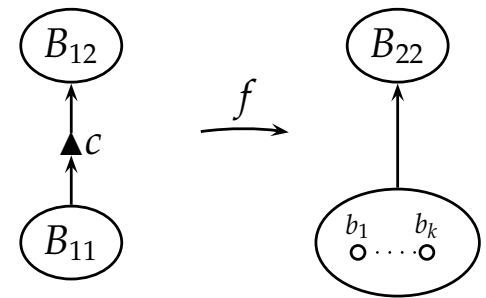
let $b_{1}$ be this element;

- if non of the elements of $B_{11}$ has a loop and non of the elements of $B_{21}$ has a loop, then let $b_{1}$ be any element of $B_{21}$. 
The rest of the elements of $B_{21}$ we denote by $b_{2}, \ldots, b_{k}, k \geq 3$.

Since $\left(\mathbf{B}_{1}, \mathbf{B}_{2}, f, c\right)$ is a minimal witness, it follows that $\left(\mathbf{B}_{1} \backslash\left\{f^{-1}\left(b_{1}\right)\right\}, \mathbf{B}_{2} \backslash\right.$ $\left\{b_{1}\right\}, f\left\lceil_{B_{1} \backslash\left\{f^{-1}\left(b_{1}\right)\right\}}, c\right)$ is not a witness, so there exists a $d_{1} \in A$ such that

$$
f_{1}(x)= \begin{cases}f(x), & \text { if } x \in B_{1} \backslash\left\{f^{-1}\left(b_{1}\right)\right\} \\ d_{1}, & \text { if } x=c\end{cases}
$$

is an extension of $f \Upsilon_{B_{1} \backslash\left\{f^{-1}\left(b_{1}\right)\right\}}$ to $\mathbf{B}_{1} \backslash\left\{f^{-1}\left(b_{1}\right)\right\} \cup\{c\}$. Analogously, we conclude that $\left(\mathbf{B}_{\mathbf{1}} \backslash\left\{f^{-1}\left(b_{2}\right)\right\}, \mathbf{B}_{\mathbf{2}} \backslash\left\{b_{2}\right\}, f \uparrow_{B_{1} \backslash\left\{f^{-1}\left(b_{2}\right)\right\}}, c\right)$ is not a witness, so there exists a $d_{2} \in A$ such that

$$
f_{2}(x)= \begin{cases}f(x), & \text { if } x \in B_{1} \backslash\left\{f^{-1}\left(b_{2}\right)\right\} \\ d_{2}, & \text { if } x=c\end{cases}
$$

is an extension of $f \Upsilon_{\mathbf{B}_{1} \backslash\left\{f^{-1}\left(b_{2}\right)\right\}}$ to $\left(\mathbf{B}_{1} \backslash\left\{f^{-1}\left(b_{2}\right)\right\}\right) \cup\{c\}$. Note that then both $d_{1}$ and $d_{2}$ have a loop, so the mapping

$$
g(x)= \begin{cases}f(x), & \text { if } x \in B_{12} \\ d_{1}, & \text { if } x=f^{-1}\left(b_{1}\right) \\ d_{2}, & \text { if } x=f^{-1}\left(b_{2}\right)\end{cases}
$$

is a homomorphism.

We claim that $\left(\mathbf{B}_{12} \cup\left\{f^{-1}\left(b_{1}\right), f^{-1}\left(b_{2}\right)\right\}, \mathbf{B}_{22} \cup\left\{d_{1}, d_{2}\right\}, g, c\right)$

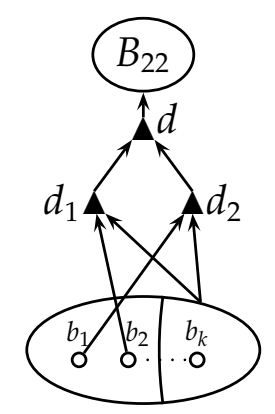
is a witness.

Suppose that it is not. Then there exists a $d \in A$ such that $g$ can be extended to

$$
\tilde{g}(x)= \begin{cases}g(x), & \text { if } x \in B_{12} \cup\left\{f^{-1}\left(b_{1}\right), f^{-1}\left(b_{2}\right)\right\} \\ d, & \text { if } x=c .\end{cases}
$$

Note that $d$ also has a loop. Moreover, $f$ can be then extended to

$$
\tilde{f}(x)= \begin{cases}f(x), & \text { if } x \in B_{1} \\ d, & \text { if } x=c,\end{cases}
$$

so $\left(\mathbf{B}_{1}, \mathbf{B}_{2}, f, c\right)$ would not be a witness - a contradiction.

We will show even more, i.e. that

$$
\left(\mathbf{B}_{12} \cup\left\{f^{-1}\left(b_{1}\right), f^{-1}\left(b_{2}\right)\right\}, \mathbf{B}_{22} \cup\left\{d_{1}, d_{2}\right\}, g, c\right) \leq\left(\mathbf{B}_{1}, \mathbf{B}_{2}, f, c\right) .
$$

For this purpose, observe the diagram

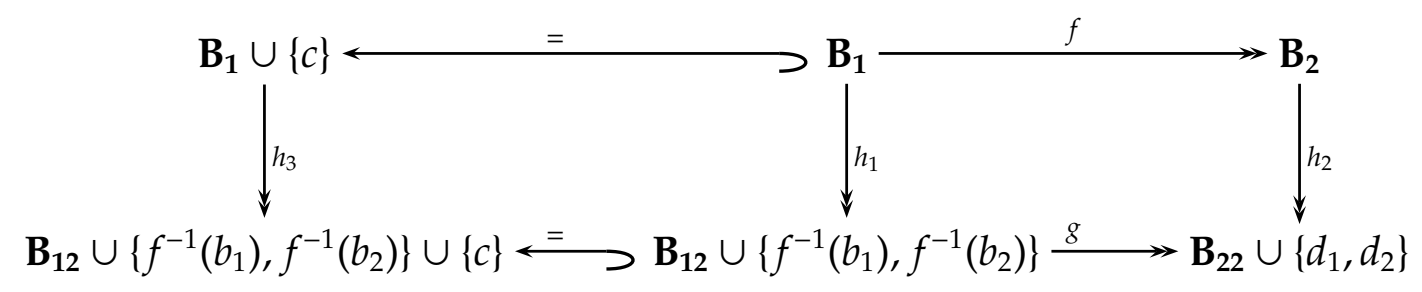


where

$$
\begin{aligned}
& h_{1}(x)= \begin{cases}x, & \text { if } x \in B_{12} \cup\left\{f^{-1}\left(b_{2}\right)\right\} \\
f^{-1}\left(b_{1}\right), & \text { otherwise, }\end{cases} \\
& h_{2}(x)= \begin{cases}x, & \text { if } x \in B_{22} \\
d_{1}, & \text { if } x=b_{1}, \\
d_{2}, & \text { if } x=b_{2}, \\
d_{1} & \text { otherwise, }\end{cases} \\
& h_{3}(x)= \begin{cases}h_{1}(x), & \text { if } x \in B_{1} \\
c, & \text { if } x=c,\end{cases}
\end{aligned}
$$

and show that $h_{1}, h_{2}$ and $h_{3}$ are homomorphisms.

$h_{1}$ is a homomorphism: Let $x, y \in B_{1}$ be such that $(x, y) \in \varrho$. Then

1. If $x=y$ and $x \in B_{12} \cup\left\{f^{-1}\left(b_{2}\right)\right\}$, then we have that $\left(h_{1}(x), h_{1}(y)\right)=(x, y) \in \varrho$.

2. If $x=y$ and $x \in B_{11} \backslash\left\{f^{-1}\left(b_{2}\right)\right\}$, then we have that $\left(h_{1}(x), h_{1}(y)\right)=\left(f^{-1}\left(b_{1}\right), f^{-1}\left(b_{1}\right)\right)$. By the definition of $b_{1}$ we can now distinguish three cases:

- $\left(b_{1}, b_{1}\right) \in \varrho$ and $\left(f^{-1}\left(b_{1}\right), f^{-1}\left(b_{1}\right)\right) \in \varrho$;

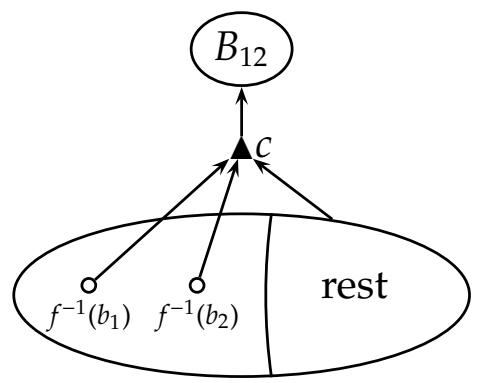

- $\left(b_{1}, b_{1}\right) \in \varrho$ and for all $i \in\{1, \ldots, k\}$, $\left(f^{-1}\left(b_{1}\right), f^{-1}\left(b_{1}\right)\right) \notin \varrho ;$

- for all $i \in\{1, \ldots, k\},\left(b_{1}, b_{1}\right) \notin \varrho$.

Note that the latter two cases cannot happen, since $x=y \in B_{11}$, so $\left(f^{-1}\left(b_{1}\right), f^{-1}\left(b_{1}\right)\right) \in \varrho$.

3. If $x \neq y$, then $x \in B_{11}$ and $y \in B_{12}$, so $h_{1}(x) \in\left\{f^{-1}\left(b_{1}\right), f^{-1}\left(b_{2}\right)\right\}$ and $h_{1}(y) \in B_{12}$, implying that $\left(h_{1}(x), h_{1}(y)\right) \in \varrho$.

$h_{2}$ is a homomorphism: Let $x, y \in B_{2}$ be such that $(x, y) \in \varrho$. Then

1. If $x=y$ and $x \in B_{22}$, then $\left(h_{2}(x), h_{2}(y)\right)=$ $(x, y) \in \varrho$.

2. If $x=y$ and $x \in B_{21}$, then

$$
\left(h_{2}(x), h_{2}(y)\right)= \begin{cases}\left(d_{1}, d_{1}\right), & \text { if } x \in B_{21} \backslash\left\{b_{2}\right\} \\ \left(d_{2}, d_{2}\right), & \text { if } x=b_{2} .\end{cases}
$$

Since $\left(d_{1}, d_{1}\right),\left(d_{2}, d_{2}\right) \in \varrho$, it follows that

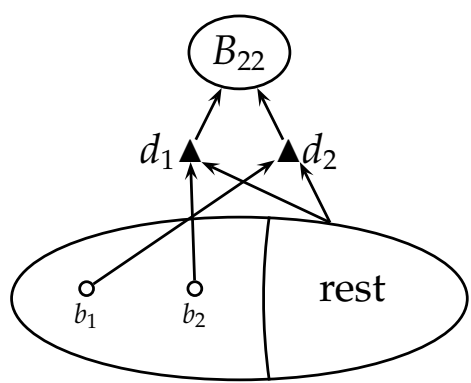
$\left(h_{2}(x), h_{2}(y)\right) \in \varrho$. 
3. If $x \neq y$, then $x \in B_{21}$ and $y \in B_{22}$, so $h_{2}(x) \in\left\{d_{1}, d_{2}\right\}$ and $h_{2}(y) \in B_{22}$, implying that $\left(h_{2}(x), h_{2}(y)\right) \in \varrho$.

$h_{3}$ is a homomorphism: Let $x, y \in B_{1} \cup\{c\}$ be such that $(x, y) \in \varrho$. Then

1. If $x, y \in B_{1}$, then $\left(h_{3}(x), h_{3}(y)\right)=\left(h_{1}(x), h_{1}(y)\right) \in \varrho$.

2. If $x=c$, then $y \in B_{12} \cup\{c\}$, so

$$
\left(h_{3}(x), h_{3}(y)\right)= \begin{cases}(c, y), & \text { if } y \in B_{12} \\ (c, c), & \text { if } y=c .\end{cases}
$$

In both cases we conclude that $\left(h_{3}(x), h_{3}(y)\right) \in \varrho$.

3. If $y=c$ and $x \in B_{11}$, then $h_{3}(x) \in B_{11}$, so $\left(h_{3}(x), h_{3}(y)\right) \in \varrho$.

It is left to show that the given diagram commutes, i.e. that $h_{2} \circ f=g \circ h_{1}$, since the other quadrangle in this diagram commutes by construction. Let $x \in B_{1}$. Then

$$
\begin{aligned}
h_{2} \circ f(x) & =h_{2}(f(x))=\left\{\begin{array}{ll}
f(x), & \text { if } f(x) \in B_{22} \\
d_{1}, & \text { if } f(x)=b_{1} \\
d_{2}, & \text { if } f(x)=b_{2} \\
d_{1}, & \text { otherwise }
\end{array}= \begin{cases}f(x), & \text { if } x \in B_{12} \\
d_{1}, & \text { if } x=f^{-1}\left(b_{1}\right) \\
d_{2,} & \text { if } x=f^{-1}\left(b_{2}\right) \\
d_{1}, & \text { otherwise }\end{cases} \right. \\
& =\left\{\begin{array}{ll}
g(x), & \text { if } x \in B_{12} \\
g(x), & \text { if } x=f^{-1}\left(b_{1}\right) \\
g(x), & \text { if } x=f^{-1}\left(b_{2}\right) \\
d_{1}, & \text { otherwise }
\end{array}= \begin{cases}g(x), & \text { if } x \in B_{12} \cup\left\{f^{-1}\left(b_{1}\right), f^{-1}\left(b_{2}\right)\right\} \\
d_{1}, & \text { otherwise. }\end{cases} \right.
\end{aligned}
$$

On the other hand,

$$
\begin{aligned}
g \circ h_{1}(x)=g\left(h_{1}(x)\right) & = \begin{cases}g(x), & \text { if } x \in B_{12} \cup\left\{f^{-1}\left(b_{1}\right), f^{-1}\left(b_{2}\right)\right\} \\
g\left(f^{-1}\left(b_{1}\right)\right), & \text { otherwise. }\end{cases} \\
& = \begin{cases}g(x), & \text { if } x \in B_{12} \cup\left\{f^{-1}\left(b_{1}\right), f^{-1}\left(b_{2}\right)\right\} \\
d_{1}, & \text { otherwise. }\end{cases}
\end{aligned}
$$

Hence, the given diagram commutes. Thus, (1) holds, implying that $\left(\mathbf{B}_{1}, \mathbf{B}_{2}, f, c\right)$ cannot be a minimal witness, which leads to a contradiction. Hence, $B_{21}$ has at most two elements.

Case 2. Let $c$ be loopless. We will first show that if $B_{21}$ has at least one loopless element, then it has at most two elements. Suppose to the contrary that $B_{21}$ has at least three elements. Let $b_{1}$ be a loopless element. We choose an element $b_{2}$ in the following manner: 
- if $B_{21}$ contains an element that has a loop and its preimage under $f$ also has a loop, then let $b_{2}$ be this element;

- if non of the elements of $B_{11}$ has a loop and $B_{21}$ contains an element that has a loop, then
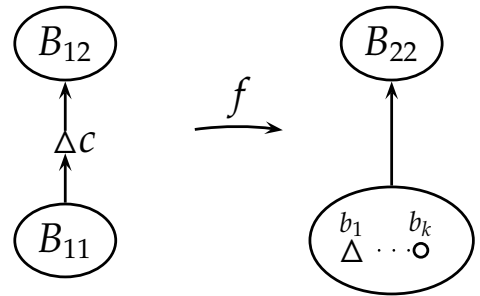
let $b_{2}$ be this element;

- if non of the elements of $B_{11}$ has a loop and non of the elements of $B_{21}$ has a loop, then let $b_{2}$ be any element of $B_{21}$.

The rest of the elements of $B_{21}$ we denote by $b_{2}, \ldots, b_{k}, k \geq 3$.

Since $\left(\mathbf{B}_{1}, \mathbf{B}_{2}, f, c\right)$ is a minimal witness, it follows that $\left(\mathbf{B}_{1} \backslash\left\{f^{-1}\left(b_{2}\right)\right\}, \mathbf{B}_{2} \backslash\right.$ $\left\{b_{2}\right\}, f\left\lceil_{B_{1} \backslash\left\{f^{-1}\left(b_{2}\right)\right\}}, c\right)$ is not a witness, so there exists a $d \in A$ such that

$$
f_{2}(x)= \begin{cases}f(x), & \text { if } x \in B_{1} \backslash\left\{f^{-1}\left(b_{2}\right)\right\} \\ d, & \text { if } x=c\end{cases}
$$

is an extension of $f\left\lceil_{B_{1} \backslash\left\{f^{-1}\left(b_{1}\right)\right\}}\right.$ to $\left(\mathbf{B}_{\mathbf{1}} \backslash\left\{f^{-1}\left(b_{1}\right)\right\}\right) \cup\{c\}$.

Note that then the mapping

$$
g(x)= \begin{cases}f(x), & \text { if } x \in B_{12} \cup\left\{f^{-1}\left(b_{2}\right)\right\} \\ d, & \text { if } x=f^{-1}\left(b_{1}\right)\end{cases}
$$

is a homomorphism. We claim that $\left(\mathbf{B}_{12} \cup\right.$ $\left.\left\{f^{-1}\left(b_{1}\right), f^{-1}\left(b_{2}\right)\right\}, \mathbf{B}_{22} \cup\left\{d, b_{2}\right\}, g, c\right)$ is a witness.

Suppose that it is not. Then there exists a

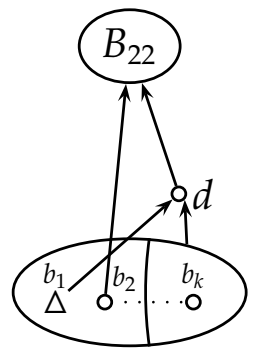
$\tilde{d} \in A$ such that $g$ can be extended to

$$
\tilde{g}(x)= \begin{cases}g(x), & \text { if } x \in B_{12} \cup\left\{f^{-1}\left(b_{1}\right), f^{-1}\left(b_{2}\right)\right\} \\ \tilde{d}, & \text { if } x=c .\end{cases}
$$

Moreover, $f$ can be then extended to

$$
\tilde{f}(x)= \begin{cases}f(x), & \text { if } x \in B_{1} \\ \tilde{d}, & \text { if } x=c,\end{cases}
$$

so $\left(\mathbf{B}_{1}, \mathbf{B}_{2}, f, c\right)$ would not be a witness - a contradiction.

We will show even more, i.e. that

$$
\left(\mathbf{B}_{12} \cup\left\{f^{-1}\left(b_{1}\right), f^{-1}\left(b_{2}\right)\right\}, \mathbf{B}_{22} \cup\left\{d, b_{2}\right\}, g, c\right) \leq\left(\mathbf{B}_{1}, \mathbf{B}_{2}, f, c\right) .
$$


For this purpose, observe the diagram

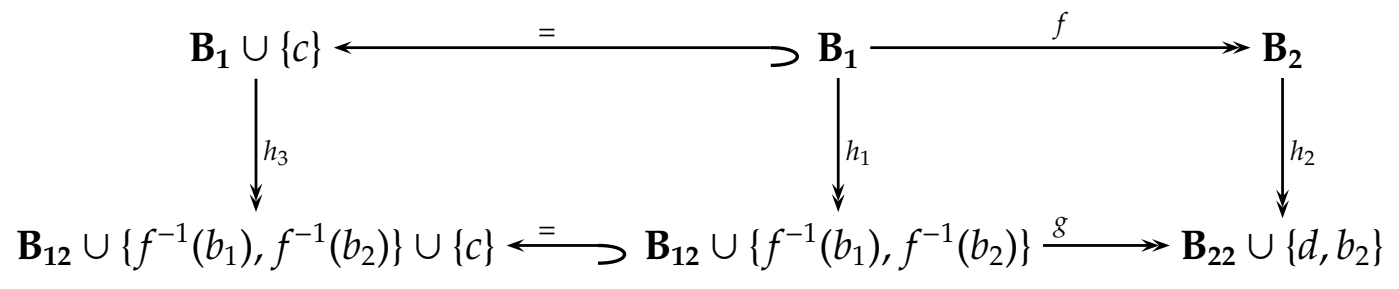

where

$$
\begin{aligned}
& h_{1}(x)= \begin{cases}x, & \text { if } x \in B_{12} \cup\left\{f^{-1}\left(b_{1}\right)\right\} \\
f^{-1}\left(b_{2}\right), & \text { otherwise, }\end{cases} \\
& h_{2}(x)= \begin{cases}x, & \text { if } x \in B_{22} \\
d, & \text { if } x=b_{1}, \\
b_{2} & \text { otherwise, }\end{cases} \\
& h_{3}(x)= \begin{cases}h_{1}(x), & \text { if } x \in B_{1} \\
c, & \text { if } x=c,\end{cases}
\end{aligned}
$$

and show that $h_{1}, h_{2}$ and $h_{3}$ are homomorphisms.

$h_{1}$ is a homomorphism: Let $x, y \in B_{1}$ be such that $(x, y) \in \varrho$. Then

1. If $x=y$ and $x \in B_{12} \cup\left\{f^{-1}\left(b_{1}\right)\right\}$, then we have that $\left(h_{1}(x), h_{1}(y)\right)=(x, y) \in \varrho$.

2. If $x=y$ and $x \in B_{11} \backslash\left\{f^{-1}\left(b_{1}\right)\right\}$, then we have that $\left(h_{1}(x), h_{1}(y)\right)=\left(f^{-1}\left(b_{2}\right), f^{-1}\left(b_{2}\right)\right)$. Since in $B_{11} \backslash\left\{f^{-1}\left(b_{1}\right)\right\}$ exists an element with a loop, we have that $\left(f^{-1}\left(b_{2}\right), f^{-1}\left(b_{2}\right)\right) \in \varrho$, so it follows $\left(h_{1}(x), h_{1}(y)\right)=(x, y) \in \varrho$.

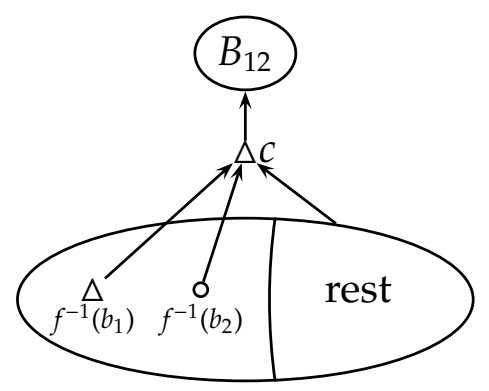

3. If $x \neq y$, then $x \in B_{11}$ and $y \in B_{12}$, so $h_{1}(x) \in$ $\left\{f^{-1}\left(b_{1}\right), f^{-1}\left(b_{2}\right)\right\}$ and $h_{1}(y) \in B_{12}$, implying that $\left(h_{1}(x), h_{1}(y)\right) \in \varrho$.

$h_{2}$ is a homomorphism: Let $x, y \in B_{2}$ be such that $(x, y) \in \varrho$. Then 
1. If $x=y$ and $x \in B_{22}$, then $\left(h_{2}(x), h_{2}(y)\right)=$ $(x, y) \in \varrho$.

2. If $x=y$ and $x \in B_{21}$, then $x \neq b_{1}$, so we obtain $\left(h_{2}(x), h_{2}(y)\right)=\left(b_{2}, b_{2}\right)$. Since in $B_{21}$ there exist an element with a loop, it follows that $\left(b_{2}, b_{2}\right) \in$ $\varrho$. Since $\left(d_{1}, d_{1}\right) \in \varrho$ and $\left(d_{2}, d_{2}\right) \in \varrho$, we have that $\left(h_{2}(x), h_{2}(y)\right) \in \varrho$.

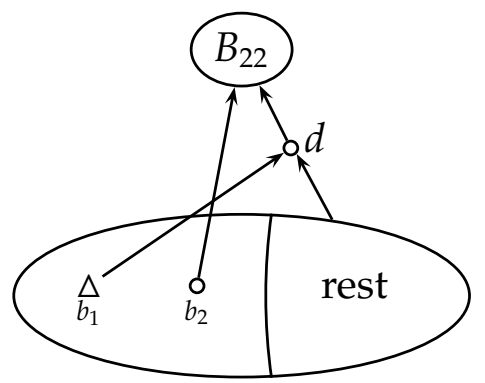

3. If $x \neq y$, then $x \in B_{21}$ and $y \in B_{22}$, so $h_{2}(x) \in\left\{d, b_{2}\right\}$ and $h_{2}(y) \in B_{22}$, implying that $\left(h_{2}(x), h_{2}(y)\right) \in \varrho$.

$h_{3}$ is a homomorphism: Let $x, y \in B_{1} \cup\{c\}$ be such that $(x, y) \in \varrho$. Then

1. If $x, y \in B_{1}$, then $\left(h_{3}(x), h_{3}(y)\right)=\left(h_{1}(x), h_{1}(y)\right) \in \varrho$.

2. If $x=c$, then $y \in B_{12}$, so $\left(h_{3}(x), h_{3}(y)\right)=\left(c, h_{1}(y)\right)=(x, y) \in \varrho$.

3. If $y=c$, then $x \in B_{11}$, so $h_{3}(x) \in B_{11}$, and $\left(h_{3}(x), h_{3}(y)\right) \in \varrho$.

It is left to show that the given diagram commutes, i.e. that $h_{2} \circ f=g \circ h_{1}$, since the other quadrangle in this diagram commutes by construction. Let $x \in B_{1}$. Then

$$
h_{2} \circ f(x)=h_{2}(f(x))= \begin{cases}f(x), & \text { if } f(x) \in B_{22} \\ d, & \text { if } f(x)=b_{1} \\ b_{2}, & \text { otherwise }\end{cases}
$$

On the other hand,

$$
\begin{aligned}
g \circ h_{1}(x)=g\left(h_{1}(x)\right) & = \begin{cases}g(x), & \text { if } x \in B_{12} \cup\left\{f^{-1}\left(b_{1}\right)\right\} \\
g\left(f^{-1}\left(b_{2}\right)\right), & \text { otherwise. }\end{cases} \\
& = \begin{cases}f(x), & \text { if } f(x) \in B_{22} \\
d, & \text { if } f(x)=b_{1} \\
b_{2}, & \text { otherwise }\end{cases}
\end{aligned}
$$

Hence, the given diagram commutes. Thus, (2) holds, implying that $\left(\mathbf{B}_{1}, \mathbf{B}_{2}, f, c\right)$ cannot be a minimal witness, which leads to a contradiction. Hence, if $B_{21}$ has a loopless element, then it has at most two elements.

We continue our analysis under the assumption that all elements of $B_{21}$ have a loop. Then $B_{21}$ has at least two elements. We claim that $B_{21}$ can then have $k$ elements, for any $k \geq 2$. For the proof, consider the following structure:

Let $k \in \mathbb{N}, k \geq 2$. Let, further, $A=\left\{c, a_{1}, \ldots, a_{k}, b_{1}, \ldots, b_{k}, d_{1}, \ldots, d_{k}\right\}$. We define $\varrho$ as

$$
\begin{gathered}
\varrho=\left\{\left(a_{i}, a_{i}\right) \mid i \in\{1, \ldots, k\}\right\} \cup\left\{\left(b_{i}, b_{i}\right) \mid i \in\{1, \ldots, k\}\right\} \cup\left\{\left(a_{i}, c\right) \mid i \in\{1, \ldots, k\}\right\} \cup \\
\left\{\left(b_{j}, d_{i}\right) \mid i \in\{1, \ldots, k\}, j \in\{1, \ldots, k\} \backslash\{i\}\right\} \cup\left\{\left(a_{j}, d_{i}\right) \mid i \in\{1, \ldots, k\}\right\}
\end{gathered}
$$


We show that $\left(\mathbf{B}_{1}, \mathbf{B}_{2}, f, c\right)$ is a minimal witness, where $B_{1}=\left\{a_{1}, \ldots, a_{k}\right\}, B_{2}=\left\{b_{1}, \ldots, b_{k}\right\}$ and $f: a_{i} \mapsto b_{i}, i \in\{1, \ldots, k\}$. Note first that $\left(\mathbf{B}_{1}, \mathbf{B}_{2}, f, c\right)$ has no proper subwitness. So, every witness $\left(\tilde{\mathbf{B}}_{1}, \tilde{\mathbf{B}}_{2}, \tilde{f}, \tilde{c}\right) \leq\left(\mathbf{B}_{1}, \mathbf{B}_{2}, f, c\right)$ has to be a homomorphic image of $\left(\mathbf{B}_{1}, \mathbf{B}_{2}, f, c\right)$. Note

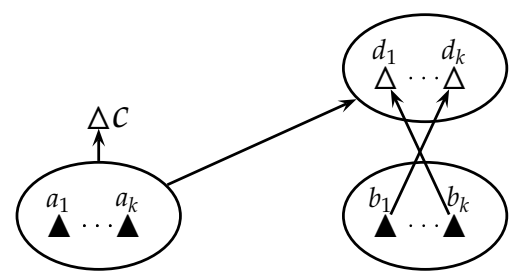
that $\tilde{\mathbf{B}}_{2}$ has to be a substructure without a weak center. Since it has to be a homomorphic image of $\mathbf{B}_{2}$, it must consist of elements from loops. Reasoning in the same way, we conclude that $\tilde{\mathbf{B}}_{1}$ also consists of elements with loops. From this it follows that $\tilde{B}_{1}, \tilde{B}_{2} \subseteq\left\{a_{1}, \ldots, a_{k}, b_{1}, \ldots, b_{k}\right\}$. If for some $b_{i}$, we have that $b_{i} \notin \tilde{B}_{2}$, then $\tilde{B}_{2}$ has the weak center $d_{i}$, so $\tilde{B}_{2} \supseteq\left\{b_{1}, \ldots, b_{k}\right\}$, and, therefore, $\tilde{B}_{2}=\left\{b_{1}, \ldots, b_{k}\right\}$. Analogously, $\tilde{B}_{1}$ has to be a $k$-element subset of $\left\{a_{1}, \ldots, a_{k}, b_{1}, \ldots, b_{k}\right\}$ that is not containing all $b_{i}{ }^{\prime}$ s. The minimal witness obtained in this way is isomorphic to $\left(\mathbf{B}_{1}, \mathbf{B}_{2}, f, c\right)$, so $\left(\mathbf{B}_{1}, \mathbf{B}_{2}, f, c\right)$ is indeed a minimal witness.

The claim can be analogously shown for $B_{22}$.

From the previous claims we can now conclude:

Claim 6. If for some $i=1,2, B_{1 i}$ is not empty, then it has as many elements as $B_{2 i}$, and among them at least as many loopless as $B_{2 i}$.

Possible minimal witnesses are given in the following table:

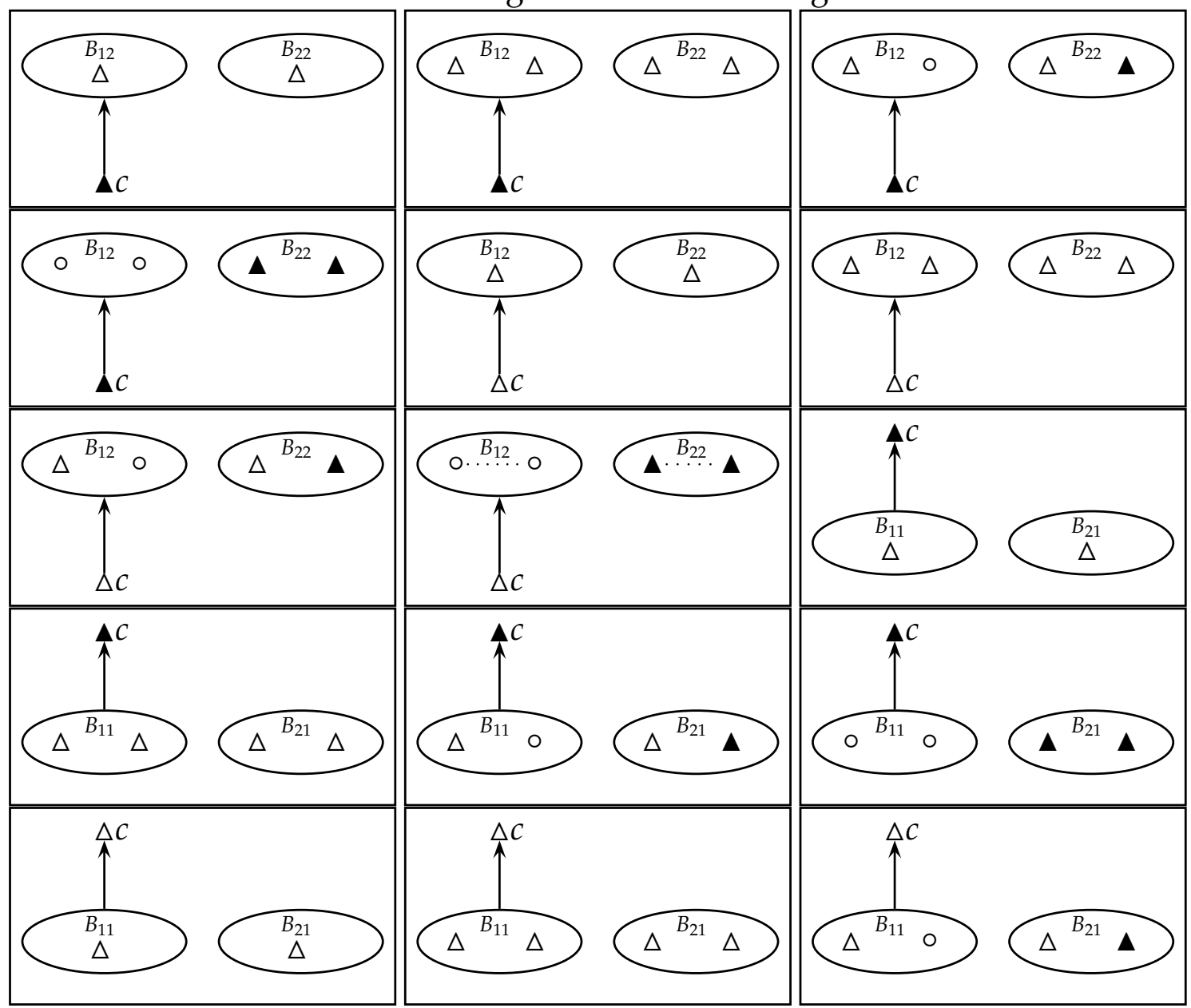


Chapter 3. Homomorphism-homogeneous relational structures

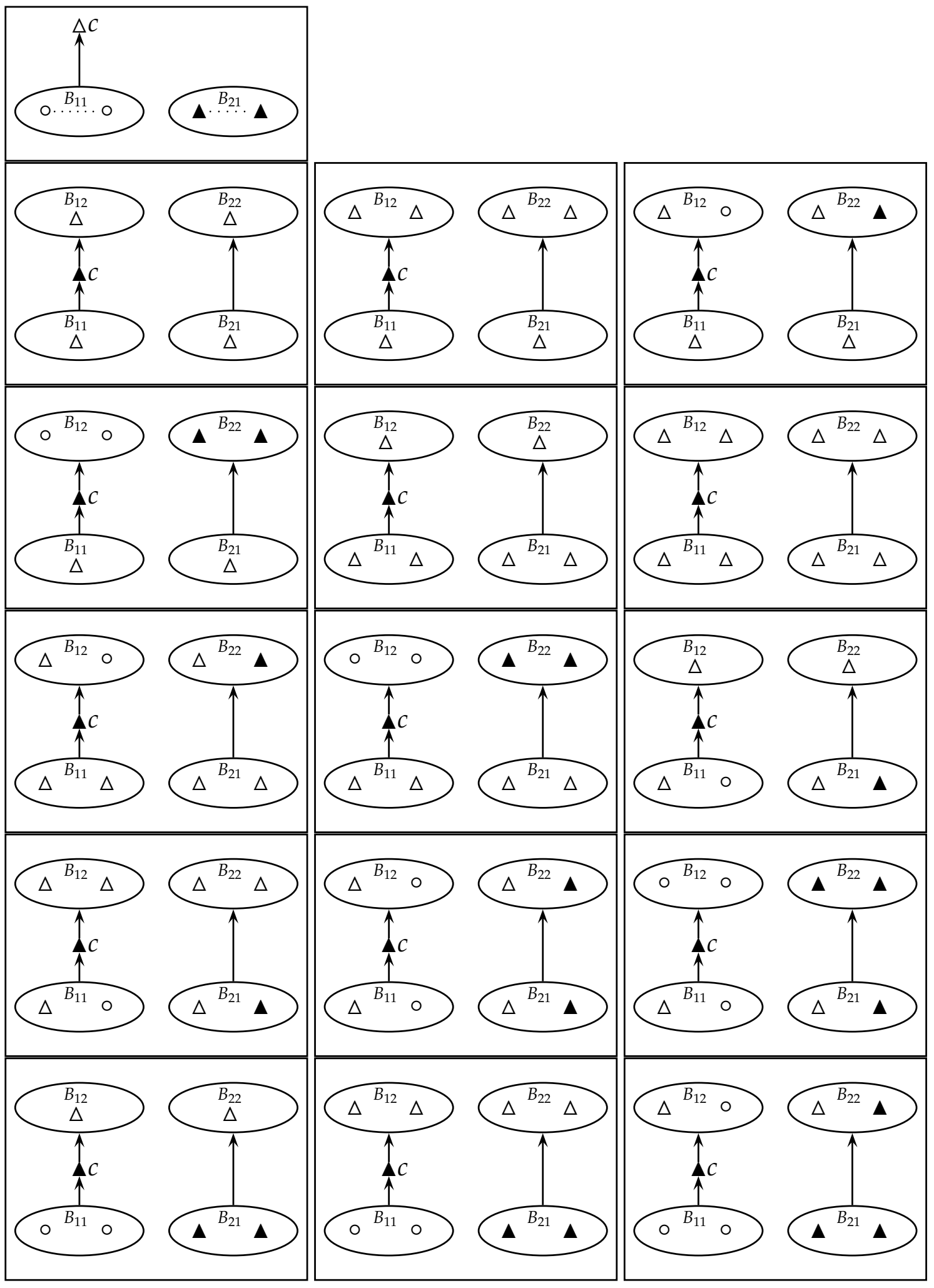




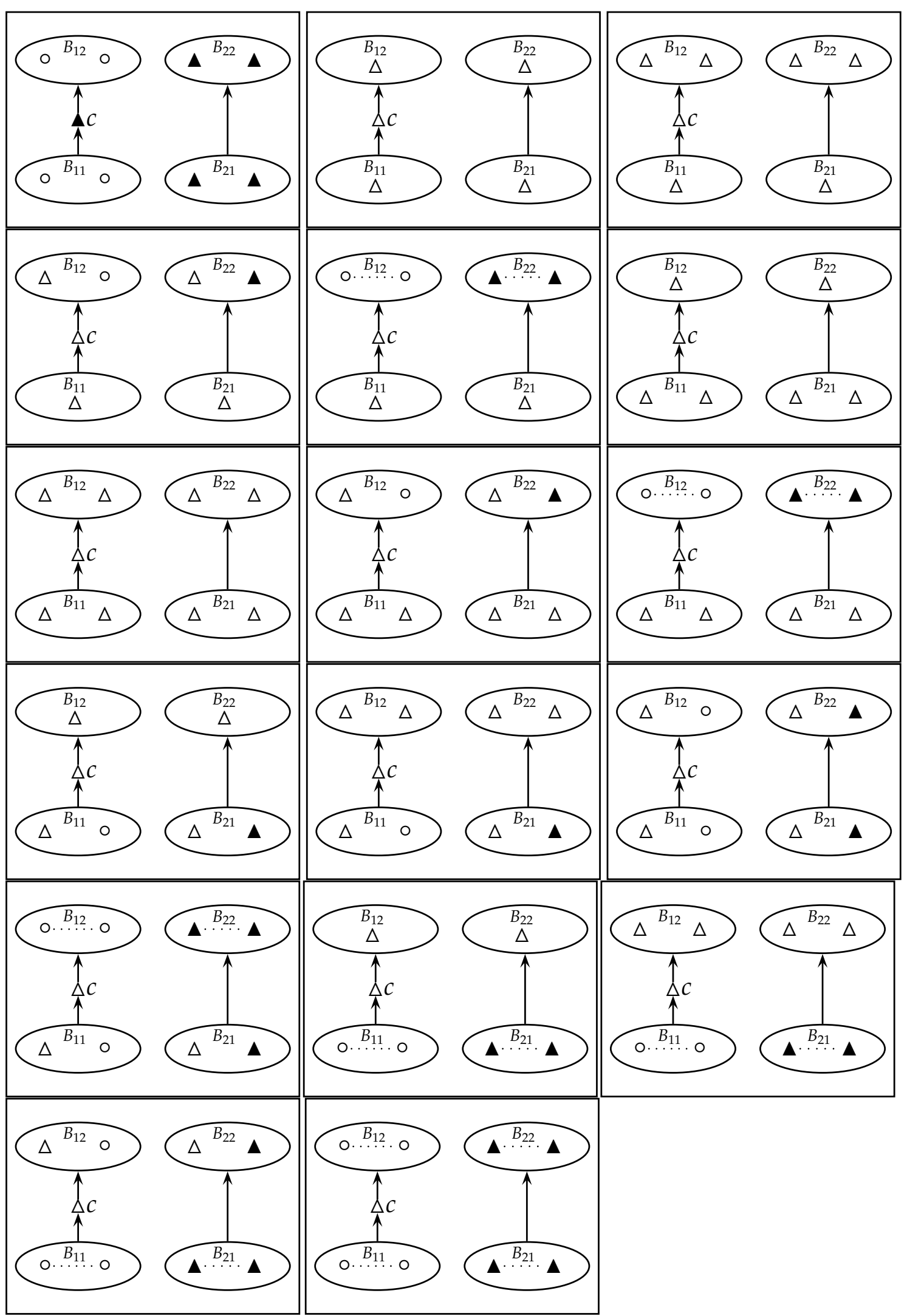

In order to demonstrate how these minimal witnesses can be used for the 
concrete characterization of homomorphism-homogeneous relations let us repeat the classification of homomorphism-homogeneous posets (cf. [28]).

Example. Let $(A, \varrho)$ be a poset. Instead of $(x, y) \in \varrho$, we will usually write $x \leq_{\varrho} y$. It is not hard to see that the relevant minimal witness for posets are as follows:

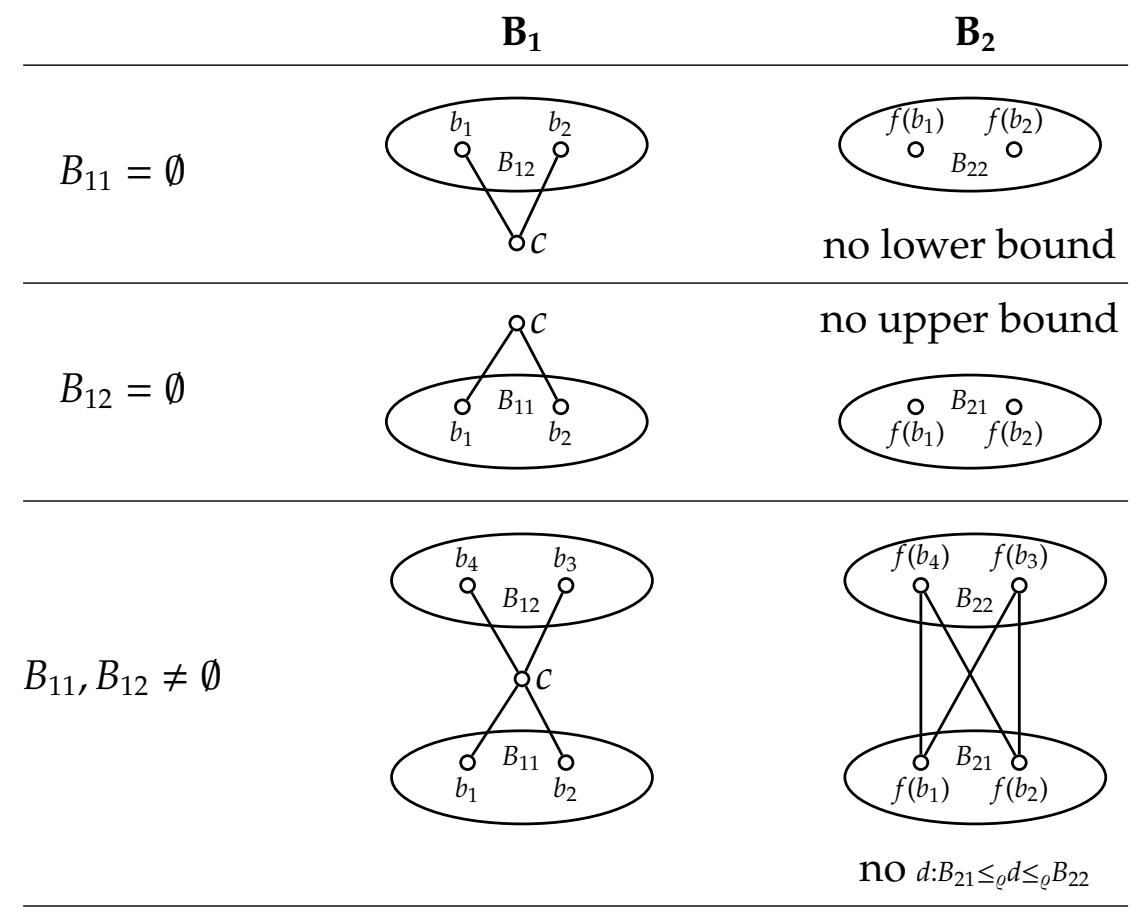

First of all, observe that chains contain no witnesses, so they all have weak onepoint extension property. If a poset is not a chain, then it contains at least two non-comparable elements. We now take in the consideration such a poset, and distinguish the following two cases:

Case 1. A poset contains no substructure of the shape

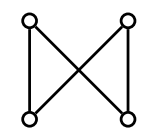

1. If no two-element antichain has an upper bound, and no two-element antichain has a lower bound, then such a poset is a disjoint union of chains.

2. If all two-element antichains have an upper bound, and no two-element antichain has a lower bound, then every two-element antichain has precisely one minimal upper bound, so such a poset is a dual tree.

3. If no two-element antichain has an upper bound, and all two-element antichains have a lower bound, then every two-element antichain has precisely one maximal upper bound, and such a poset is a tree. 
4. If all two-element antichains have an upper bound, and all two-element antichains have a lower bound, then every two-element antichain has precisely one minimal upper bound and one maximal lower bound, so such a poset is a tree "glued" with a dual tree by identifying vertices.

Case 2. A poset contains a substructure of the shape<smiles>C1OC12CO2</smiles>

Then there exists a two-element antichain that has an upper bound, and, therefore, all two-element antichains have an upper bound. Also, there exists a twoelement antichain that has a lower bound, so all two-element antichains have a lower bound. Since a poset contains no minimal witness, it follows that either no

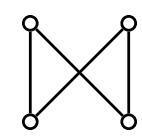

can be extended to

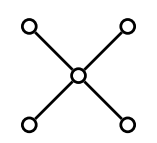

i.e. a poset is $X_{5}$-free, or in other words, it is a tree "glued" with a dual tree by connecting with edges, or it follows that all

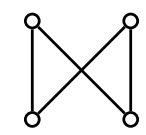

can be extended to

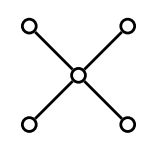

i.e. a poset is $X_{5}$-dense.

3.2.2. Minimal witnesses for transitive relations. After characterizing all minimal witnesses for antisymmetric transitive relations, we turn our attention to transitive relations. Let $\mathbf{A}=(A, \varrho)$ be such that $\varrho$ is a transitive relation. Note that every transitive relation has a retract that is an antisymmetric transitive relation. In order to see this, define an equivalence relation $\simeq$ as $\left(\varrho \cap \varrho^{-1}\right) \cup \Delta_{A}$ and a set $B$ as a transversal of $A / \simeq$. Then $\varrho \cap B^{2}$ is an antisymmetric transitive relation on $B$. Now we define $r: \mathbf{A} \rightarrow \mathbf{B}$ by mapping an $x \in A$ to the representative of $[x]_{\simeq}$ in $B$. It is not hard to see that $r$ is a retraction. Let $\iota$ be the right inverse embedding of $r$. 
Our goal is to show that $\mathbf{A}$ has the one-point extension property if and only if $\mathbf{B}$ does. From 2.3.2(Lemma 1), it is already known that if $\mathbf{A}$ has this property, then so does every retract of $\mathbf{A}$. Thus, it suffices to show that if $\mathbf{B}$ has this property, so does $\mathbf{A}$, or, in other words, if $\mathbf{A}$ has a witness, then $\mathbf{B}$ also has one.

Suppose that $\left(\mathbf{B}_{1}, \mathbf{B}_{2}, f, c\right)$ is a witness in $\mathbf{A}$. We will show that $\left(r\left(\mathbf{B}_{1}\right), r\left(\mathbf{B}_{2}\right), g, r(c)\right)$ is a witness in $\mathbf{B}$, where $g$ is such that the diagram

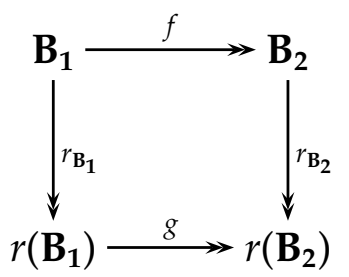

commutes and $r_{\mathbf{B}_{1}}, r_{\mathbf{B}_{2}}$ are corresponding restrictions of $r$. First of all, we show that such a $g$ exists. Note that $r\left(\mathbf{B}_{1}\right)$ is a retract of $\mathbf{B}_{1}$, so take the identical embedding $\iota_{\mathbf{B}_{1}}: r\left(\mathbf{B}_{1}\right) \rightarrow \mathbf{B}_{1}$ and define $g:=r_{\mathbf{B}_{2}} \circ f \circ \iota_{\mathbf{B}_{1}}$, where $\iota_{\mathbf{B}_{1}}$ is a restriction of $\iota$ on $B_{1}$. Then $g$ is a homomorphism and a surjection by construction.

Suppose that $\left(r\left(\mathbf{B}_{1}\right), r\left(\mathbf{B}_{2}\right), g, r(c)\right)$ for such a chosen $g$ is not a witness. Then there exists a $d \in A$ such that

$$
\tilde{g}(x)= \begin{cases}g(x), & \text { if } x \in r\left(B_{1}\right) \\ d, & \text { if } x=r(c),\end{cases}
$$

is an extension of $g$ to $r\left(\mathbf{B}_{\mathbf{1}}\right) \cup\{r(c)\}$. We will show that $f$ also has an extension, in particular, that

$$
\tilde{f}(x)= \begin{cases}f(x), & \text { if } x \in B_{1} \\ d, & \text { if } x=c,\end{cases}
$$

extends $f$ to $\mathbf{B}_{1} \cup\{c\}$. To show that $\tilde{f}$ is a homomorphism, w.l.o.g. take $(c, x) \in \varrho$, such that $x \in B_{1}$. Then $(r(c), r(x)) \in \varrho$. On the other hand, $r(c), r(x) \in r\left(B_{1}\right) \cup\{r(c)\}$, so $(d, \tilde{g}(r(c)))=(\tilde{g}(r(c)), \tilde{g}(r(x))) \in \varrho$. However,

$$
\tilde{g}(r(x))=\left(r_{\mathbf{B}_{2}} \circ f \circ \iota_{\mathbf{B}_{1}}\right)(r(x))=\left(r_{\mathbf{B}_{2}} \circ f\right)(r(x))=r_{\mathbf{B}_{2}}(f(r(x))),
$$

so $\left(d, r_{\mathbf{B}_{2}}(f(r(x)))\right) \in \varrho$. Note that if $f(r(x))$ is loopless, then, $r_{\mathbf{B}_{2}}(f(r(x)))=f(r(x))$, so $(d, f(r(x))) \in \varrho$. On the other hand, if $f(r(x))$ has a loop, then $\left(r_{\mathbf{B}_{2}}(f(r(x))), f(r(x))\right) \in$ $\varrho$, so by transitivity, we obtain $(d, f(r(x))) \in \varrho$. Moreover, if $x$ is loopless, then $r(x)=$ $x$, so $(d, f(x)) \in \varrho$. However, if $x$ has a loop, then $(r(x), x) \in \varrho$, so $(f(r(x)), f(x)) \in \varrho$, and finally, $(\tilde{f}(c), \tilde{f}(x))=(d, f(x)) \in \varrho$.

Hence, $\tilde{f}$ is indeed an extension, so $\left(\mathbf{B}_{1}, \mathbf{B}_{2}, f, c\right)$ cannot be a witness. Contradiction.

Corollary. A quasi-order has the one-point extension property if and only if it is the full preimage of a partial order with the one point extension property. 
3.2.3. Minimal witnesses for tolerance relations. Let $\mathbf{A}=(A, \varrho)$ be such that $\varrho$ is a tolerance relation, i.e. $\varrho$ is a reflexive symmetric binary relation. It is well known that every tolerance relation can be represented in the form of a graph with all possible loops, so we will use this opportunity to make this study more friendly to the reader. To every tolerance relation will be associated a drawing of its corresponding simple graph (meaning that we leave out all loops). From 3.1.4(Proposition 1) we already know that if $\left(\mathbf{B}_{1}, \mathbf{B}_{2}, f, c\right)$ is a minimal witness in $\mathbf{A}$, then $f$ is bijective. Since $c$ is a weak center of $\mathbf{B}_{\mathbf{1}}$, it follows that for every $b \in B_{1}$ holds that $(c, b),(b, c) \in \varrho$.

Note that tolerance relations that are binary central relations have no witnesses at all, since every substructure has a weak center (e.g. any central element), so they always have the one-point extension property. Therefore, we will concentrate in the se-

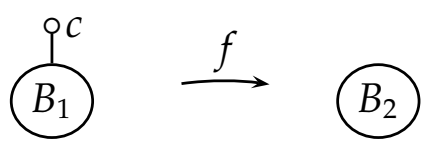
quel on noncentral tolerance relations.

We turn our attention now to a potential minimal witness $\left(\mathbf{B}_{1}, \mathbf{B}_{2}, f, c\right)$ that has the smallest cardinality of $B_{1}$. Then it is of the shape (1).

Using this observation, we will describe those tolerance relations that do not contain the above described minimal witness.

Proposition 1. Let $\mathbf{A}=(A, \varrho)$ a rela-

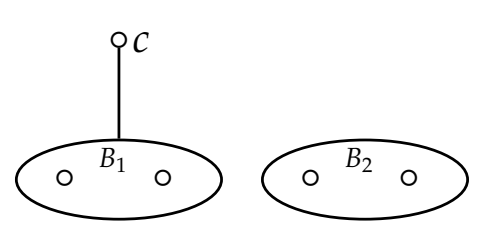
tional structure where $\varrho$ is a tolerance relation. Then $\mathbf{A}$ does not have a minimal witness of the shape (1) if and only if one of the following two is true:

- $\varrho$ is an equivalence relation or

- the graph of $\varrho$ is connected and of diameter at most 2.

Proof. Note that neither equivalence relations nor the connected graphs of diameter at most 2 have this witness. Suppose now that A does not have a minimal witness of the shape (1). We distinguish the following two cases:

Case 1. The graph $G$ of $\varrho$ is disconnected. We will show that $\varrho$ has to be transitive. Suppose that it is not. Then there exist elements $a_{1}, a_{2} \in A$ such that $\left(a_{1}, c\right),\left(c, a_{2}\right) \in \varrho$, but $\left(a_{1}, a_{2}\right) \notin \varrho$. Since $G$ is disconnected, it has at least two connected components. Let $b_{1}$ and $b_{2}$ be the elements from different components. Then for $B_{1}:=\left\{a_{1}, a_{2}\right\}, B_{2}:=\left\{b_{1}, b_{2}\right\}$ and $f: a_{i} \mapsto b_{i}, i=1,2$, we have that $\left(\mathbf{B}_{1}, \mathbf{B}_{2}, f, c\right)$ is a witness of the shape (1) - a contradiction. Hence, $\varrho$ is transitive, and, therefore, an equivalence relation.

Case 2. The graph $G$ of $\varrho$ is connected. Suppose that it has diameter greater than 2. Then there exist $b_{1}$ and $b_{2}$ of distance 3. Let $a_{1}$ and $a_{2}$ be of distance 2, i.e. there exists a $c$ such that $\left(a_{1}, c\right),\left(c, a_{2}\right) \in \varrho$. But now, for $B_{1}:=\left\{a_{1}, a_{2}\right\}, B_{2}:=\left\{b_{1}, b_{2}\right\}$ and 
$f: a_{i} \mapsto b_{i}, i=1,2$, we have that $\left(\mathbf{B}_{1}, \mathbf{B}_{2}, f, c\right)$ is a witness of the shape (1) - a contradiction. Hence, $G$ is of diameter at most 2 .

Our next goal is to describe minimal witnesses that are not of the shape (1).

Proposition 2. Let $\mathbf{D}=(D, \sigma)$ be such that $\sigma$ is a noncentral tolerance relation and $D$ is finite. Then there exists a structure $\mathbf{A}$ with a minimal witness $\left(\mathbf{B}_{\mathbf{1}}, \mathbf{B}_{\mathbf{2}}, f, c\right)$ such that $\mathbf{B}_{2} \cong \mathbf{D}$.

Proof. Suppose $D=\left\{d_{1}, \ldots, d_{k}\right\}$ and let $\tilde{D}:=\left\{\tilde{d}_{1}, \ldots, \tilde{d}_{k}\right\}$ be such that $D \cap \tilde{D}=\emptyset$ and $|D|=|\tilde{D}|=k$. Finally, let $d$ be such that $d \notin D \cup \tilde{D}$. Define

$$
\begin{aligned}
A & :=D \cup \tilde{D} \cup\{d\} \\
\varrho_{1} & :=\left\{\left(d, \tilde{d}_{i}\right) \mid i \in\{1, \ldots, k\}\right\} \\
\varrho_{2} & :=\left\{\left(d_{i}, \tilde{d}_{j}\right) \mid i, j \in\{1, \ldots, k\} \wedge i \neq j\right\} \\
\varrho & :=\sigma \cup \varrho_{1} \cup \varrho_{1}^{-1} \cup \varrho_{2} \cup \varrho_{2}^{-1} \cup \Delta_{A}
\end{aligned}
$$

Further, define $B_{1}:=\left\{d, d_{2}, \ldots, d_{k}\right\}$ and $B_{2}:=\left\{d_{1}, \ldots, d_{k}\right\}$. Let $c:=\tilde{d}_{1}$ and let

$$
f: \mathbf{B}_{1} \rightarrow \mathbf{B}_{2}: x \mapsto \begin{cases}d_{1}, & \text { if } x=d \\ x, & \text { otherwise, }\end{cases}
$$

Then $\left(\mathbf{B}_{1}, \mathbf{B}_{2}, f, c\right)$ is a witness of the structure $\mathbf{A}=(A, \varrho)$. In the next step we show that every minimal witness of $\mathbf{A}$ is of the shape $\left(\tilde{\mathbf{B}}_{1}, \mathbf{B}_{2}, \tilde{f}, \tilde{c}\right)$. It is enough to show that every substructure $\mathbf{S}$ of $\mathbf{A}$ without a weak center contains $\mathbf{B}_{\mathbf{2}}$. Indeed, if there exists a $d_{i} \in B_{2}$ such that $d_{i} \notin S$, then $\tilde{d}_{i}$ is a weak center of $S$.

3.2.4. Homomorphism-homogeneous tolerance relations. Our goal now is to describe a practicable way for the constructive enumeration of all homomorphism-homogeneous tolerance relations on a given finite set $A$. In the previous subsection we have shown that homomorphism-homogeneous tolerance relations with a disconnected corresponding graph are equivalence relations, so it is left to characterize those that have a connected graph. This can be done by filtering out all those relations that are not homomorphism-homogeneous. Moreover, we have to check just those tolerance relations whose corresponding graph is of diameter 2. Note that all tolerance relations whose graph is of diameter 1 are central relations, and, therefore, homomorphism-homogeneous. Let $\varrho$ be a tolerance relation on $A$. In order to show that graph $G$ of $\varrho$ is not homomorphism-homogeneous, we have to show that it contains a minimal witness. In the following we describe a strategy for finding minimal witnesses:

For this purpose, we introduce a quasi-order on substructures of $\mathbf{A}=(A, \varrho)$. For substructures $\mathbf{D}_{1}, \mathbf{D}_{2}$ of $\mathbf{A}$ we say that $\mathbf{D}_{\mathbf{1}}$ is below $\mathbf{D}_{2}$ and write $\mathbf{D}_{\mathbf{1}} \leq \mathbf{D}_{\mathbf{2}}$ if there exists a substructure $\mathbf{D}_{3}$ of $\mathbf{D}_{2}$ and a surjective homomorphism $h: \mathbf{D}_{3} \rightarrow \mathbf{D}_{1}$.

It is not hard to see that the relation $\leq$ is a quasi-order and that the corresponding equivalence relation is the isomorphism relation. Moreover, if $\left(\mathbf{B}_{1}, \mathbf{B}_{2}, f, c\right)$ 
is a minimal witness of $\mathbf{A}$, then $\mathbf{B}_{\mathbf{2}}$ is a minimal substructure with respect to $\leq$ among all those substructures that do not have a weak center. Therefore, when we search for minimal witnesses in $\mathbf{A}$, it is natural to find first among all substructures without a weak center the minimal ones with respect to $\leq$ (i.e. $\leq$-minimal substructures). Once we found them, we look for their bijective homomorphic preimages in A that have a weak center. If this search is successful, then we know that $\mathbf{A}$ is not homomorphism-homogeneous.

The problem of finding bijective homomorphic preimages of substructures in A can be reduced to the problem of finding bijective homomorphic images of substructures in $\overline{\mathbf{A}}=\left(A, A^{2} \backslash \varrho\right)$ :

Lemma. Let $\left(A_{1}, \varrho_{1}\right)$ and $\left(A_{2}, \varrho_{2}\right)$, where $\varrho_{1} \subseteq A_{1}^{2}$ and $\varrho_{2} \subseteq A_{2}^{2}$, be relational structures and $f: A_{1} \rightarrow A_{2}$ a bijective homomorphism. Let $\overline{\varrho_{1}}=A_{1}^{2} \backslash \varrho_{1}$ and $\overline{\varrho_{2}}=A_{2}^{2} \backslash \varrho_{2}$. Then $f^{-1}: A_{2} \rightarrow A_{1}$ is a homomorphism from $\left(A_{2}, \overline{\varrho_{2}}\right)$ to $\left(A_{1}, \overline{\varrho_{1}}\right)$.

Proof. Let $\left(a_{1}, a_{2}\right) \in \bar{\varrho}_{2}$. Since $a_{1}, a_{2} \in A_{2}$ and $f$ is bijective, it follows that there exist $b_{1}=f^{-1}\left(a_{1}\right)$ and $b_{2}=f^{-1}\left(a_{2}\right)$. We prove indirectly that $\left(b_{1}, b_{2}\right) \in \overline{\varrho_{1}}$.

If $\left(b_{1}, b_{2}\right) \notin \bar{\varrho}_{1}$, then $\left(b_{1}, b_{2}\right) \in \varrho_{1}$, so $\left(f\left(b_{1}\right), f\left(b_{2}\right)\right) \in \varrho_{2}$. On the other hand, $\left(f\left(b_{1}\right), f\left(b_{2}\right)\right)=\left(f\left(f^{-1}\left(a_{1}\right)\right), f\left(f^{-1}\left(a_{2}\right)\right)\right)=\left(a_{1}, a_{2}\right)$, so $\left(a_{1}, a_{2}\right) \in \varrho_{2}$, and, therefore, $\left(a_{1}, a_{2}\right) \notin \overline{\varrho_{2}}$, which is a contradiction.

We conclude that $f^{-1}: A_{2} \rightarrow A_{1}$ is a homomorphism from $\left(A_{2}, \overline{\varrho_{2}}\right)$ to $\left(A_{1}, \overline{\varrho_{1}}\right)$.

In general, the above described method works well only for rather small basic sets $A$, so we give a few refinements that speed up the whole process:

Note that homomorphism-homogeneous relational structures are closed with respect to retracts. Suppose that we have already computed all homomorphismhomogeneous relations on proper subsets of $\mathbf{A}$. Then we can simplify our search by computing several easily obtainable retracts of $\mathbf{A}$ and looking if they appear in the list that we have already computed. If one of them does not, then we know that $\mathbf{A}$ is not homomorphism homogeneous.

Before developing the next idea, let us set some terminology that will be used in the sequel. Given a relational structure $\mathbf{A}=(A, \varrho)$. Consider the relation $\bar{\varrho}=A^{2} \backslash \varrho$ and take the smallest equivalence relation $\theta$ that contains $\bar{\varrho}$. The equivalence classes of $\theta$ we will call components of $\mathbf{A}$. If $\mathbf{A}$ has just one component, then we call it connected.

The next idea is based on the observation that disconnected homomorphismhomogeneous tolerance relations can be composed from connected homomorphism-homogeneous tolerance relations.

Proposition 1. Let $\mathbf{A}=(A, \varrho)$ be such that $\varrho$ is a noncentral tolerance relation. Let $G_{1}, \ldots, G_{n}, n \geq 2$, be the components of $\mathbf{A}$. Then substructures $H$ of $\mathbf{A}$ without a weak center in $A$ are in one-to-one correspondence with tuples $\left(H_{1}, \ldots, H_{n}\right)$, where $H_{i}$ is a substructure of $\mathbf{G}_{\mathbf{i}}$ without a weak center in $G_{i}, i=1, \ldots, n$. Moreover, this correspondence is given by

$$
\begin{aligned}
H & \mapsto\left(H \cap G_{1}, \ldots H \cap G_{n}\right) \\
\left(H_{1}, \ldots, H_{n}\right) & \mapsto H_{1} \cup \cdots \cup H_{n} .
\end{aligned}
$$


Proof. Let $\mathbf{H}$ be a substructure of $\mathbf{A}$ without a weak center in $A$. First of all, if $H$ contains exactly one element from some component $G_{i}$, then this element of this component is a weak center of $H$ in $A$. Moreover, if for some $i$ we have that $H \cap G_{i}=\emptyset$, then any element of $G_{i}$ is a weak center of $\mathbf{H}$. Therefore, $H$ must contain at least two elements from each of the components of $\mathbf{A}$. Moreover, if for some $i, H \cap G_{i}$ has a weak center in $G_{i}$, then this weak center is going to be a weak center of $H$. Hence, the components of $H$ are $H_{1}, \ldots, H_{n}$, such that $\mathbf{H}_{i} \leq \mathbf{G}_{\mathbf{i}}$, $H_{i}$ has at least two elements and $H_{i}$ has no weak center in $G_{i}, i=1, \ldots, n$. On the other hand, for every choice of $H_{1}, \ldots, H_{n}$, such that $\mathbf{H}_{i} \leq \mathbf{G}_{\mathbf{i}}, H_{i}$ has at least two elements and $H_{i}$ has no weak center in $G_{i}, i=1, \ldots, n$, gives a substructure $\mathbf{H}$ without a weak center in $A$ such that $H_{i}$ 's are components of $H$. Indeed, if $H$ would have a weak center, then this weak center would be an element of some $G_{i}$, and, therefore, a weak center of the corresponding $H_{i}$, which is in a contradiction with a choice of $H_{i}$. Thus, the desired one-to-one correspondence is established.

Proposition 2. Let $\mathbf{A}=(A, \varrho)$ be a homomorphism-homogeneous relational structure such that $\varrho$ is a noncentral tolerance relation and let $G_{1}, \ldots, G_{n}, n \geq 2$ be the components of $\mathbf{A}$. Then a substructure $\mathbf{H}$ that consist of the components $G_{1}, \ldots, G_{n-1}$ is also homomorphism-homogenous.

Proof. Let $G_{1}, \ldots, G_{n}, n \geq 2$, be the components of $\mathbf{A}$ and let $H=G_{1} \cup \cdots \cup G_{n-1}$. Suppose that $\mathbf{H}$ has a witness $\left(\mathbf{B}_{1}, \mathbf{B}_{2}, f, c\right)$. Without loss of generality, we can assume that $f$ is bijective. Clearly, the components of $H$ are $G_{1}, \ldots, G_{n-1}$. Since $B_{21}$ is a substructure of $\mathbf{H}$ that has no weak center, from Proposition 1 we know that $\mathbf{B}_{\mathbf{2}}$ corresponds to the $(n-1)$-tuple $\left(B_{21}, \ldots, B_{2, n-1}\right)$ where $\mathbf{B}_{\mathbf{2} \mathbf{i}}=\mathbf{B}_{\mathbf{2}} \cap \mathbf{G}_{\mathbf{i}}$ is a substructure of $\mathbf{G}_{\mathbf{i}}$ that has no weak center in $G_{i}$. Further, there exists a component $G_{i}(i \in\{1, \ldots, n-1\})$ such that $c \in G_{i}$. In the next step we take a substructure $\mathbf{B}_{\mathbf{2}}$ of $\mathbf{G}_{\mathbf{n}}$ without a weak center in $G_{n}$ and define the mapping $\hat{f}: B_{1} \cup B_{2 n} \rightarrow B_{21} \cup \cdots \cup B_{2 n}$ where

$$
\hat{f}: x \mapsto \begin{cases}f(x), & \text { if } x \in B_{1} \\ x, & \text { if } x \in B_{2 n}\end{cases}
$$

It is not hard to see that $\hat{f}$ is a bijective homomorphism. Moreover, $c$ is a weak center of $B_{1} \cup B_{2 n}$ and using Proposition 1 we obtain that $B_{21} \cup \cdots \cup B_{2 n}$ has no weak center in $A$. Thus, $\left(\mathbf{B}_{1} \cup \mathbf{B}_{2 n}, \mathbf{B}_{21} \cup \cdots \cup \mathbf{B}_{2 n}, \hat{f}, c\right)$ is a witness in $\mathbf{A}-$ contradiction.

Corollary. Let $\mathbf{A}=(A, \varrho)$ be a homomorphism-homogeneous relational structure such that $\varrho$ is a noncentral tolerance relation and let $G_{1}, \ldots, G_{n}, n \geq 2$ be the components of A. Then every $\mathbf{G}_{\mathbf{i}}, i \in\{1, \ldots, n\}$, is homomorphism-homogeneous.

We can now test the homomorphism homogeneity of relational structures $\mathbf{A}=(A, \varrho)$ where $\varrho$ is a noncentral tolerance relation, by removing one component of $A$ at the time and checking if the structure obtained in such a way is 
also homomorphism-homogeneous. In Appendix A the results of a computer enumeration of homomorphism-homogeneous tolerance relations is presented.

3.2.5. Examples of homomorphism-homogeneous tolerance relations. We finish our study of homomorphism-homogeneous tolerance relations by describing some infinite families of examples. We will denote by $S_{k}$ the star-graph on $k$ vertices (see (1)). The complete graph on $k$ vertices will be denoted by $K_{k}$.

Example 1. Let $G$ be a simple graph such that all connected components of $G$ are stargraphs, i.e. every component is isomorphic to some $S_{k}$. Let $A$ be the vertex set of $G$ and let $\varrho$ be the complement of the adjacency relation of $G$. Then $\mathbf{A}=(A, \varrho)$ is homomorphismhomogeneous.

Proof. First of all, it is obvious that the vertex sets of the connected components of $G$ are the components of $\mathbf{A}$. Denote them by $A_{1}, \ldots, A_{n}$. Then the minimal substructure without a weak center in A corresponds by 3.2.4(Proposition 1) to a tuple $\left(H_{1}, \ldots, H_{n}\right)$ where each $\mathbf{H}_{\mathbf{i}}, i=1, \ldots, n$, is a minimal substructure of $\mathbf{A}_{\mathbf{i}}$ with no weak center in $A_{i}$. Note that for every $i \in\{1, \ldots, n\}$ the restriction of $\varrho$ to $A_{i}$ is an equivalence relation with two equivalence classes, where at least one of the classes is a singleton. It is not hard to see that the transversals of these equivalence relations define minimal substructures of $\mathbf{A}_{\mathbf{i}}$ without a weak center in $A_{i}$. Thus, a minimal substructure of $\mathbf{A}$ without a weak center in $A$ is a union of transversals of $\varrho \uparrow_{A_{i}}$, for $i=1, \ldots, n$. So, all minimal substructures of $\mathbf{A}$ without a weak center in $A$ are mutually isomorphic and, therefore, they are $\leq$-minimal.

We turn our attention now to the bijective homomorphic preimages of described $\leq$-minimal substructures of $\mathbf{A}$. Let $H=\left(H, \varrho \uparrow_{H}\right)$ be one of them. Then $H^{2} \backslash \varrho \uparrow_{H}$ is the adjacency relation of the subgraph $G_{H}$ of $G$ induced by $H$. Then $G_{H} \cong n \cdot K_{2}$ ( $n$ disjoint copies of $K_{2}$ ). Finding bijective homomorphic preimages of $\mathbf{H}$ in $\mathbf{A}$ is the same as finding bijective homomorphic images of $G_{H}$ in $G$. Note that every such an image can contain at most one edge from each of the star-graphs in $G$. Otherwise, the corresponding homomorphism is not bijective. Thus, every bijective homomorphic image of $G_{H}$ in $G$ is isomorphic to $G_{H}$, and, in particular, it contains from every star-graph an edge. These are precisely the transversals of the components of $\mathbf{A}$, and, thus, no bijective homomorphic preimage of $\mathbf{H}$ has a weak center.

Example 2. Let $G$ be a simple graph such that all connected components of $G$ are cycles of length 5 , i.e. every component is isomorphic to $C_{5}$. Let $A$ be the vertex set of $G$ and let $\varrho$ be the complement of the adjacency relation of $G$. Then $\mathbf{A}=(A, \varrho)$ is homomorphism-homogeneous. 
Proof. Again, it is obvious that the vertex sets of the connected components of $G$ are the components of $\mathbf{A}$. Denote them by $A_{1}, \ldots, A_{n}$. Then the minimal substructure without a weak center in A corre-

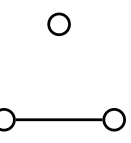
sponds again to a tuple $\left(H_{1}, \ldots, H_{n}\right)$ where each $\mathbf{H}_{\mathbf{i}}$, $i=1, \ldots, n$, is a minimal substructure of $\mathbf{A}_{\mathbf{i}}$ with no weak center in $A_{i}$. Note that for every $i \in\{1, \ldots, n\}$ the restriction of $\varrho$ to $A_{i}$ is $C_{5}$ with all possible loops. It is easy to see that minimal substructures of $\mathbf{A}_{\mathbf{i}}$ without a weak center in $A_{i}$ are then isomorphic and look as (2). A minimal substructure of $\mathbf{A}$ without a weak center in $A$ is a union of minimal substructures of $\mathbf{A}_{\mathbf{i}}$ without a weak center in $A_{i}$. Thus, all minimal substructures of $\mathbf{A}$ without a weak center in $A$ are mutually isomorphic and, therefore, they are $\leq$-minimal.

Let $H=\left(H, \varrho \uparrow_{H}\right)$ be one of the bijective homomorphic preimages of described $\leq$-minimal substructures of A. Then $H^{2} \backslash \varrho \uparrow_{H}$ is the adjacency relation of the subgraph $G_{H}$ of $G$ induced by $H$. Then $G_{H} \cong n \cdot S_{3}$ ( $n$ disjoint copies of $S_{3}$ ). As in the previous example, we find now all bijective homomorphic images of $G_{H}$ in $G$. Since $G$ contains no triangle, every bijective homomorphism maps every $S_{3}$ to another subgraph isomorphic to $S_{3}$. Two copies of $S_{3}$ cannot be mapped by a bijective homomorphism into the same copy of $C_{5}$, so we conclude that every bijective homomorphic image of $n \cdot S_{3}$ will be again isomorphic to $n \cdot S_{3}$. Thus, every bijective homomorphic preimage of $\mathbf{H}$ has no weak center.

Example 3. Let $G$ be a connected simple graph that is obtained by taking $n$ copies of $S_{3}, n \geq 3$, then taking from each copy a vertex of degree 1 and identifying them (see (3) ). Let $A$ be the vertex set of $G$ and let $\varrho$ be the complement of the adjacency relation of $G$. Then $\mathbf{A}=(A, \varrho)$ is homomorphism-homogeneous.

Proof. Let $\mathbf{U}$ be a minimal substructure of $\mathbf{A}$ without a weak center in $A$. We denote the vertices of $A$ as it is shown in (3). Note that if any of $v_{i}{ }^{\prime} \mathrm{s}$ is not contained in $U$, then corresponding $w_{i}$ is going to be a weak center of $U$. So, all $v_{i}$ 's are in $U$, but since they are inducing a substructure in $\mathbf{A}$ with a weak center in $A$, we conclude that $U$ contains more vertices. Further, denote by $G_{U}$ the subgraph of $G$ that is induced by $U$. We consider the following two cases:

Case 1. $u \in U$. Note that $\left(u, v_{i}\right) \notin \varrho$ and for all $i$ we have that $\left(w_{i}, v_{i}\right) \notin \varrho$, so the substructure induced by $u, v_{1}, \ldots v_{n}$ in $\mathbf{A}$ has no weak center in $A$, and therefore it is a minimal one. Then $\varrho \cap U^{2}$ is an equivalence relation with two equivalence classes: $\left\{v_{1}, \ldots, v_{n}\right\}$ and $\{u\}$. Note that $U$ induces a star graph $S_{n+1}$ in $G$ and consider the bijective homomorphic im-

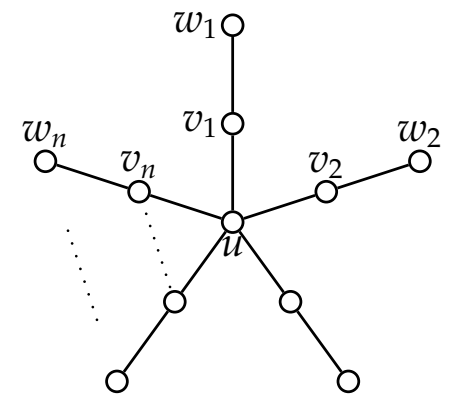
ages of $G_{U}$. The central vertex of the star 
has to be mapped to itself, since it has the highest degree in $G$ (it is unique). Therefore, the rest has to be mapped to $v_{i}$ 's, so the image is the graph itself.

Case 2. $u \notin U$. Then $U$ contains some of $w_{i}$ 's. If some $w_{j} \notin U$, then $v_{j}$ is going to be a weak center of $\mathbf{U}$ in $A$, so all $w_{i}$ 's are in $U$. Hence $U=\left\{v_{1}, \ldots, v_{n}, w_{1}, \ldots, w_{n}\right\}$ and the subgraph induced by $U$ in $G_{U}$ is isomorphic to $n \cdot K_{2}$. Since for every $i$ we have that $\left(v_{i}, w_{i}\right) \notin \varrho$ and $\left(u, v_{i}\right) \notin \varrho$, we conclude that $\mathbf{U}$ has no weak center in $A$. Consider now the bijective homomorphic images of $G_{U}$ in $G$. If $u$ does not appear in the bijective homomorphic image, then the image has to be equal to $G_{U}$. Otherwise, one of the $w_{i}{ }^{\prime} \mathrm{s}$ is not appearing in the image. But, such an image contains then the vertex set $\left\{u, v_{1}, \ldots, v_{n}\right\}$ and, thus, it has no weak center in $\mathbf{A}$.

Example 4. Let $G$ be a simple graph whose one connected component has the vertex set $V \cup W_{1} \cup \cdots \cup W_{n}, n \geq 3$, such that $V=\left\{v_{1} \ldots, v_{n}\right\}$ and the minimal degree of the vertices in $G_{V}$ is 2 in $G_{V}$. Moreover, $W_{i} \cup\left\{v_{i}\right\}$ induces a star with the center $v_{i}$, for $i=1, \ldots, n$, and all vertices of $W_{i}$ have degree 1 . The other connected components of $G$ are star-graphs. Let $A$ be the vertex set of $G$ and let $\varrho$ be the complement of the adjacency relation of $G$. Then $\mathbf{A}=(A, \varrho)$ is homomorphism-homogeneous.

Proof. Let $\mathbf{U}$ be a minimal substructure of $\mathbf{A}$ without a weak center in $A$. If for some $i, i \in\{1, \ldots, n\}$, we have that $v_{i} \notin U$, then all vertices of $W_{i}$ will be weak centers of $\mathbf{U}$ in $A$. Hence, $V \subseteq U$. Further, from every star-graph component of $G$, exactly two adjacent vertices have to be in $U$. Otherwise, $U$ has a weak center in $A$. To sum up, $G_{U}$ contains of $G_{V}$ and several copies of $K_{2}$, as many as there are star-graph connected components in $G$. It is not hard to see that $\mathbf{U}$ has no weak center in $A$. Consider now any bijective homomorphic image of $G_{U}$ in $G$. It is clear that $G_{V}$ has to be mapped to itself and that no two copies of $K_{2}$ can be mapped to the same star-graph component. Hence, the bijective homomorphic images of $G_{U}$ are isomorphic to $G_{U}$.

Example 5. Let $V=\left\{v_{1}, \ldots, v_{n}\right\}$ and let $W=\left\{w_{1}, \ldots, w_{n}\right\}, n \geq 2$. Let $A=V \cup W$ and let

$$
\varrho=V^{2} \cup W^{2} \cup\left\{\left(v_{i}, w_{i}\right) \mid i \in\{1, \ldots, n\} \cup\left\{\left(w_{i}, v_{i}\right) \mid i \in\{1, \ldots, n\}\right\} .\right.
$$

Then $\mathbf{A}=(A, \varrho)$ is homomorphism-homogeneous.

Proof. Let $G$ be the graph obtained from A by removing all loops. Then $G$ is isomorphic to the Cartesian sum $K_{2}+K_{n}$. In the following we will identify substructures of $\mathbf{A}$ with their corresponding induced subgraphs of $G$. We distinguish three possible types of minimal substructure of $\mathbf{A}$ without a weak center in $A$ :

1. $U=\left\{v_{i}, v_{j}, w_{i}, w_{j}\right\}$, where $i \neq j$ and $i, j \in\{1, \ldots, n\}$,

2. $U=\left\{v_{i}, v_{j}, w_{i}, w_{k}\right\}$, where $i, j, k$ are distinct elements of $\{1, \ldots, n\}$, 
3. $U=\left\{v_{i}, v_{j}, w_{k}, w_{l}\right\}$, where $i, j, k, l$ are distinct elements of $\{1, \ldots, n\}$.

The second case appears only for $n \geq 3$ and the third case appears only for $n \geq 4$. It is not hard to see that the first case defines the $\leq$-minimal substructures in $\mathbf{A}$ without a weak center in $A$. If $U=\left\{v_{i}, v_{j}, w_{i}, w_{j}\right\}$, then $U$ induces a cycle of length 4 in $G$. We can see at once that the possible bijective homomorphic preimages of a cycle of length 4 in $G$ are the paths of 4 vertices or two copies of $K_{2}$. However, all paths of four vertices are induced by a set of the shape as given in the second case. Moreover, all induced subgraphs of $G$ that are isomorphic to $2 \cdot K_{2}$ are induced by a set of shape as given in the third case. Hence no bijective homomorphic preimage of $\mathbf{U}$ in $\mathbf{A}$ has a weak center in $A$. 


\section{Appendix A.}

\section{Catalogue of small homomorphism-homogeneous tolerance relations}

In this appendix we will list all noncentral connected homomorphism-homogeneous tolerance relations on a basic set $A$ of cardinality at most 9 . The computations were done using the packages nauty (cf. [31]) and GAP (cf. [13]) in conjunction with GRAPE (cf. [51]). Each of the relations will be represented in the form of a connected graph. In order to draw them in the most compact and clearly organized way, we will introduce some additional rules for simplifying their graphical representation and avoiding the appearance of a large number of edges in the graphs of relations in question.

It is clear that some of relations have isomorphic graphs, so we will always put one representative in the list and, for this reason, we will avoid the labeling of the vertices of a graph. In order to make drawings as simple as possible, we make them as hierarchical diagrams.

Definition. The hierarchical diagram is a tuple $\left(\left(V_{i}\right)_{i=1}^{k},\left(E_{i}\right)_{i=1}^{k}, \tilde{E}, c, \tilde{c}\right)$ where

1. $V_{1}, \ldots, V_{k}$ are nonempty pairwise disjoint sets,

2. $E_{i} \subseteq V_{i} \times V_{i}$,

3. $\tilde{E} \subseteq\{1, \ldots, k\}^{2} \backslash \Delta_{\{1, \ldots, k\}}$,

4. $c: V \rightarrow\{w, b\}$, where $V=\bigcup_{i=1}^{k} V_{i}$, and

5. $\tilde{c}: \tilde{E} \rightarrow\{s, d\}$.

Every hierarchical diagram $\left(\left(V_{i}\right)_{i=1}^{k},\left(E_{i}\right)_{i=1}^{k}, \tilde{E}, c, \tilde{c}\right)$ defines a binary relation $\varrho$ on $V \times V$, where $V=\bigcup_{i=1}^{k} V_{i}$, as follows:

$$
\varrho:=\bigcup_{i=1}^{k} E_{i} \cup \bigcup_{\substack{i, j=1 \\ i \neq j}}^{k} E_{i j}
$$

where

$$
E_{i j}= \begin{cases}V_{i} \times V_{j}, & \text { if } \tilde{c}((i, j))=s \\ \left\{(u, v) \in V_{i} \times V_{j} \mid c(u)=w \text { or } c(v)=w\right\}, & \text { otherwise. }\end{cases}
$$


We draw hierarchical diagram in the following way: a vertex $v$ we draw as $\circ$, if $c(v)=w$, and as $\bullet$, if $c(v)=b$. The elements of $E_{i}$ are drawn as the edges between corresponding vertices. The vertices from the same $V_{i}$ we draw into an oval box. We omit the oval box if $V_{i}$ is a singleton. The elements of $\tilde{E}$ define edges between oval boxes. If for $e \in \tilde{E}$ we have that $\tilde{c}(e)=s$, then we draw a usual edge, and if $\tilde{c}(e)=d$, then we draw a dashed edge. We avoid drawing loops.

\section{A.1. Relations on 4 elements}

There is, up to isomorphism, just one connected noncentral homomorphism-homogeneous tolerance relation.

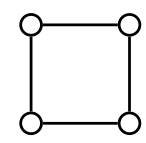

\section{A.2. Relations on 5 elements}

There are, up to isomorphism, three connected noncentral homomorphismhomogeneous tolerance relations.
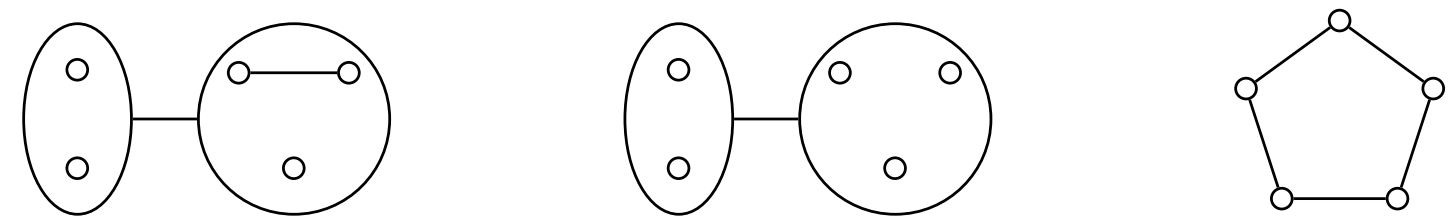

\section{A.3. Relations on 6 elements}

There are, up to isomorphism, seven connected noncentral homomorphismhomogeneous tolerance relations.
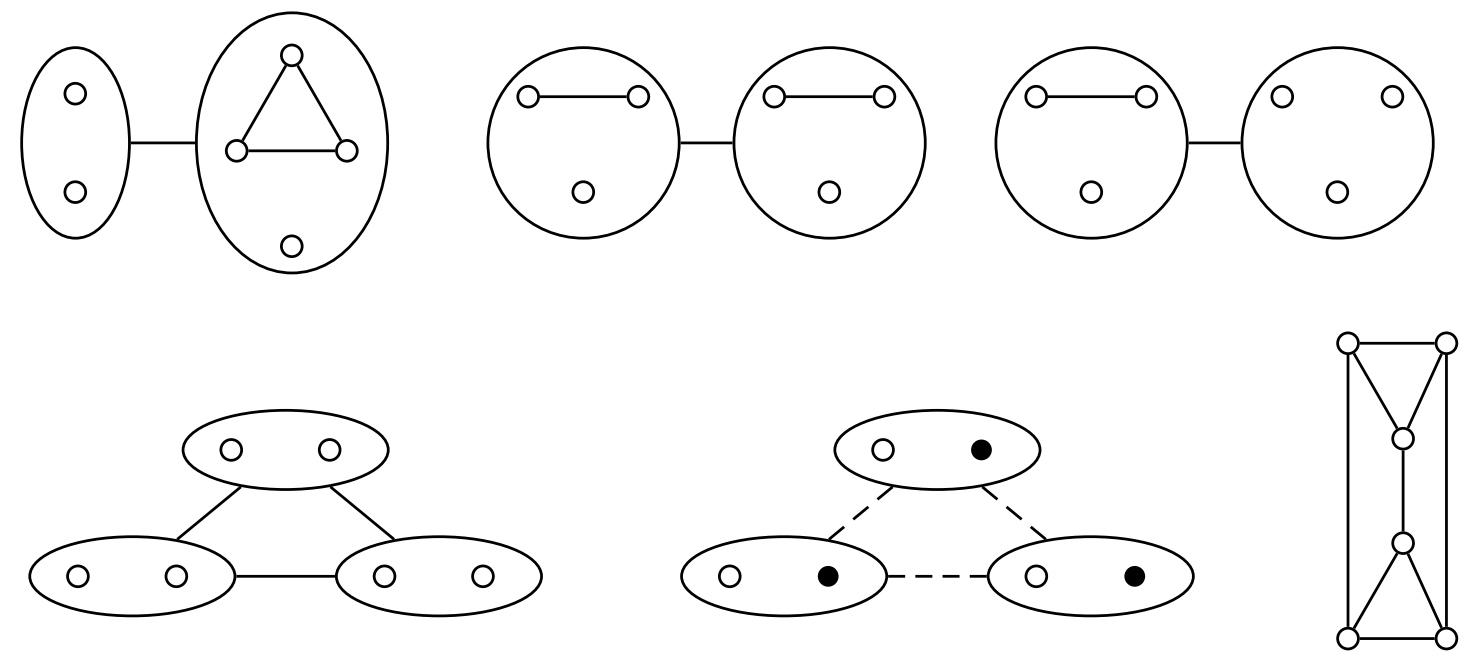


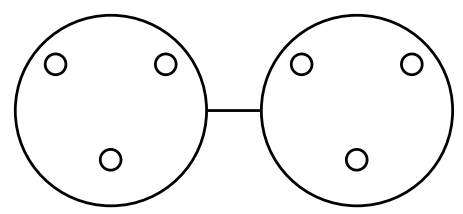

\section{A.4. Relations on 7 elements}

There are, up to isomorphism, eight connected noncentral homomorphismhomogeneous tolerance relations.
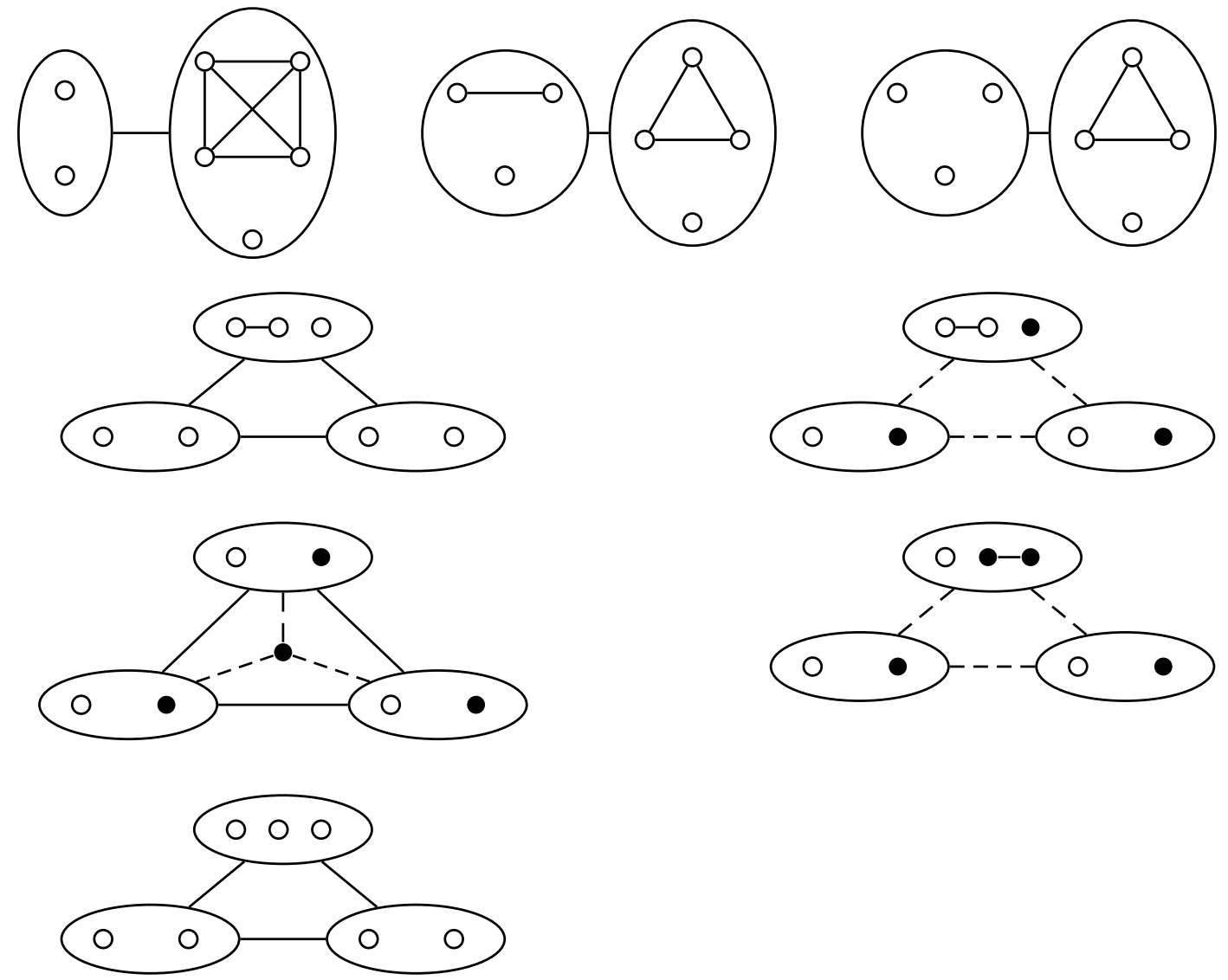

\section{A.5. Relations on 8 elements}

There are, up to isomorphism, twenty one connected noncentral homomorphismhomogeneous tolerance relations. 
Appendix A. Small homomorphism-homogeneous tolerance relations
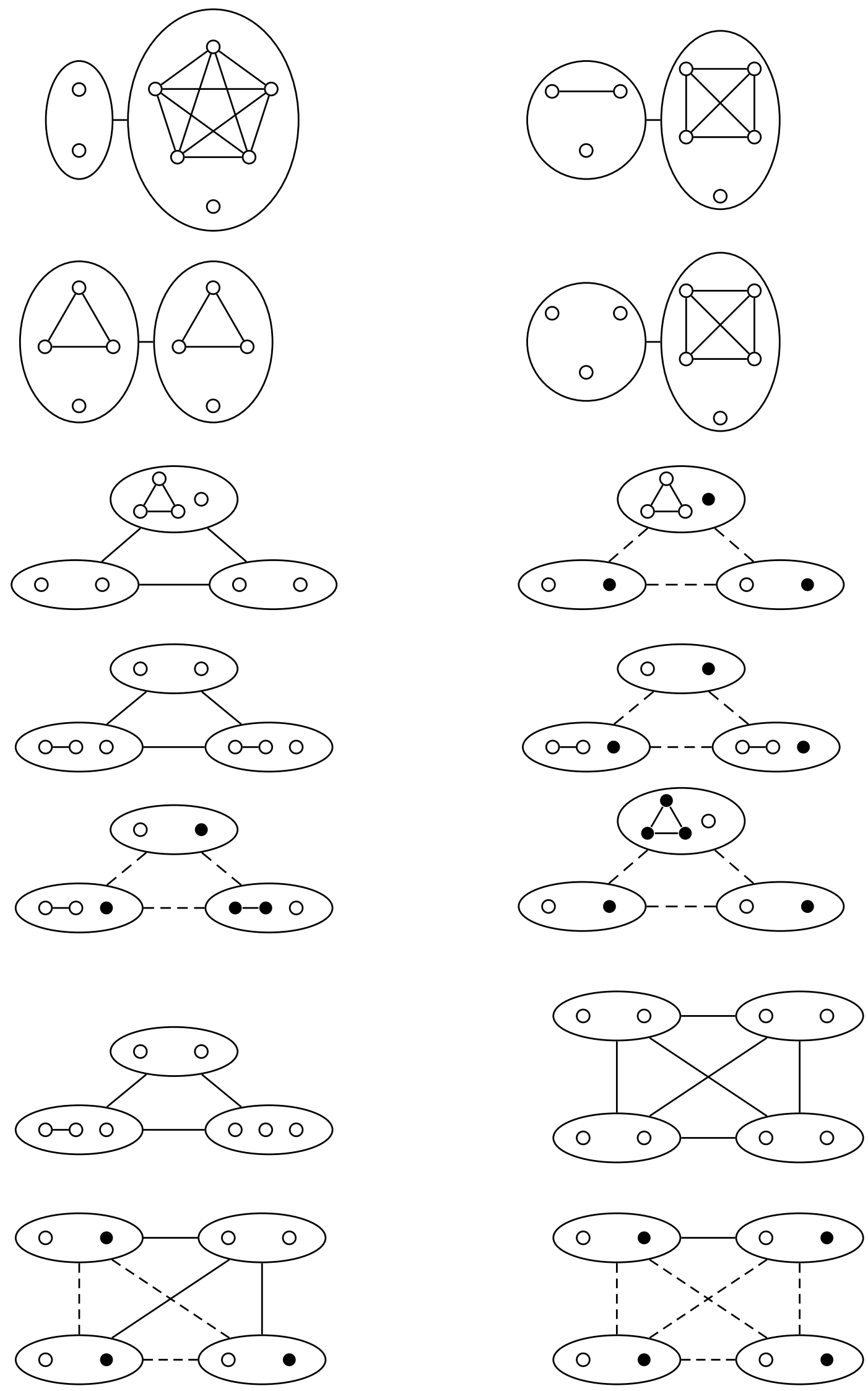

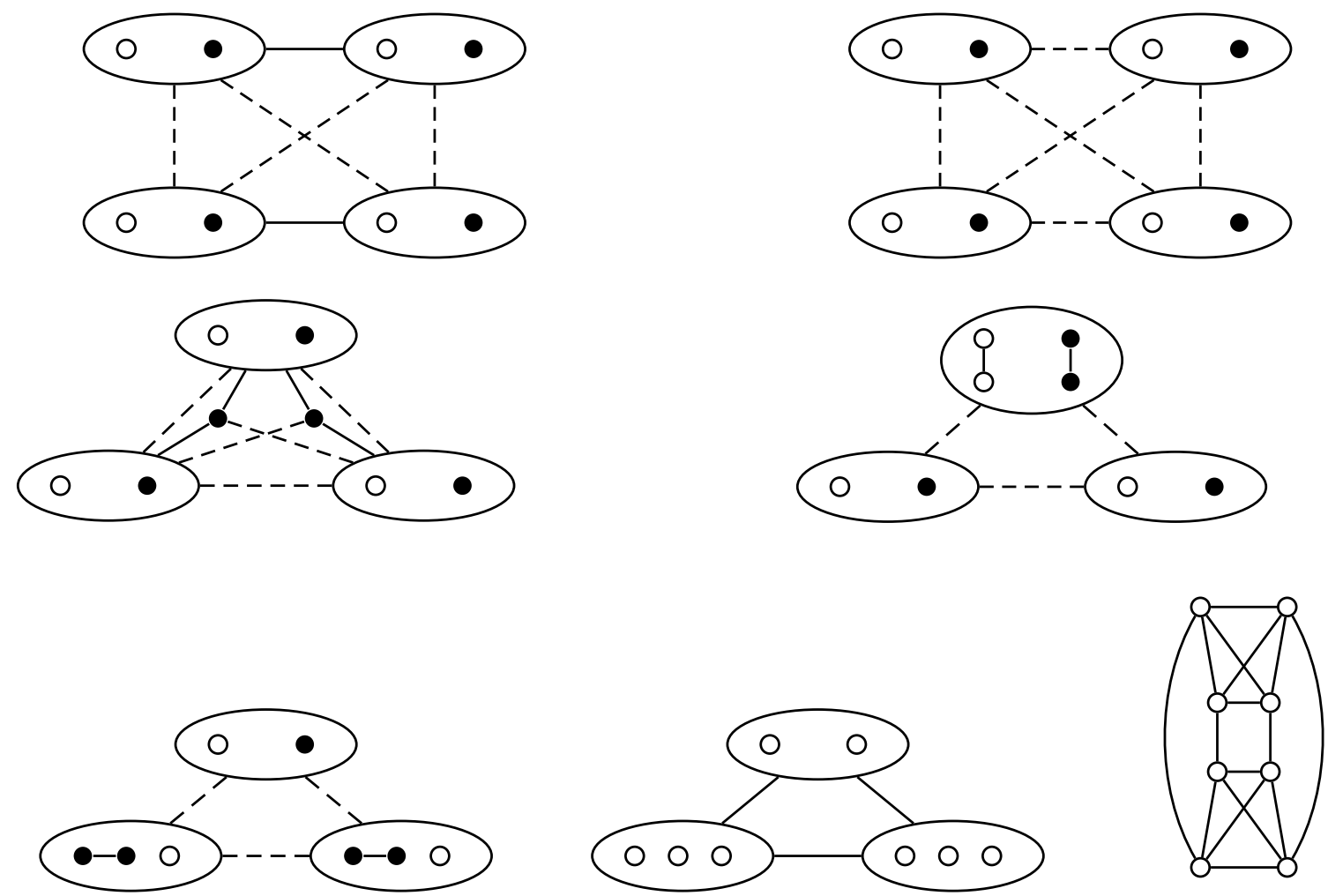

\section{A.6. Relations on 9 elements}

There are, up to isomorphism, forty seven connected noncentral homomorphismhomogeneous tolerance relations.
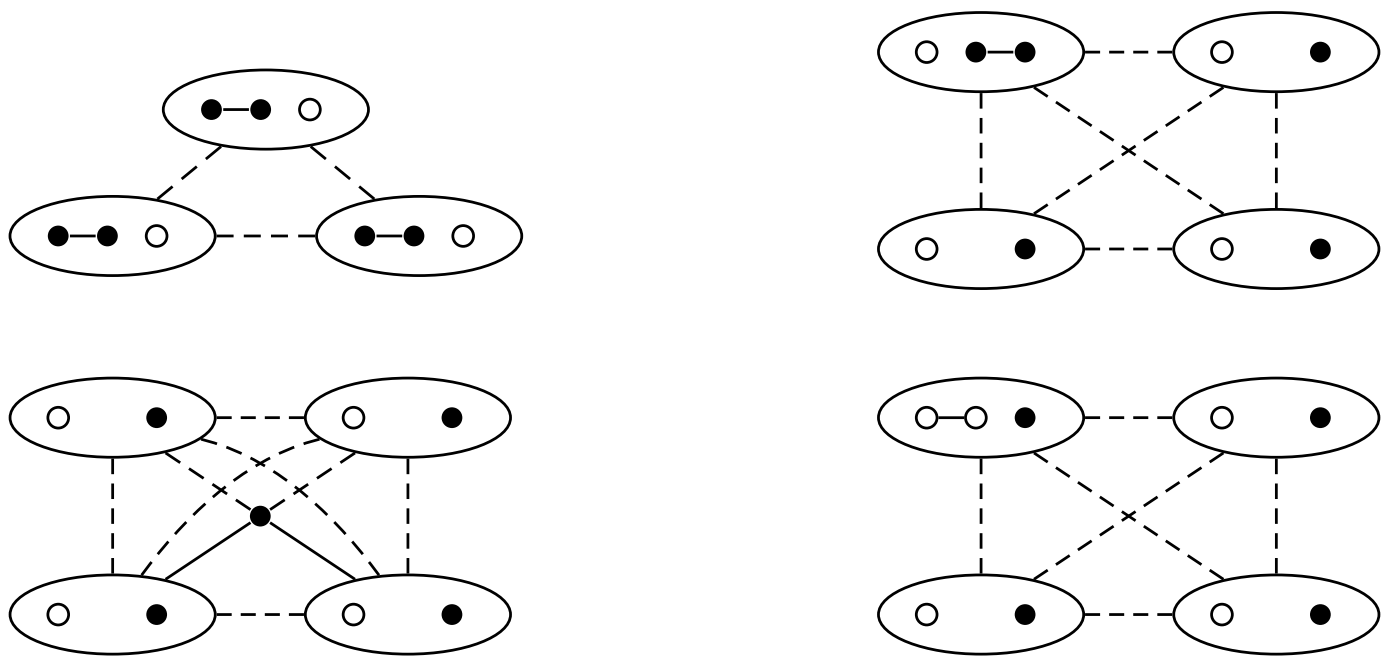

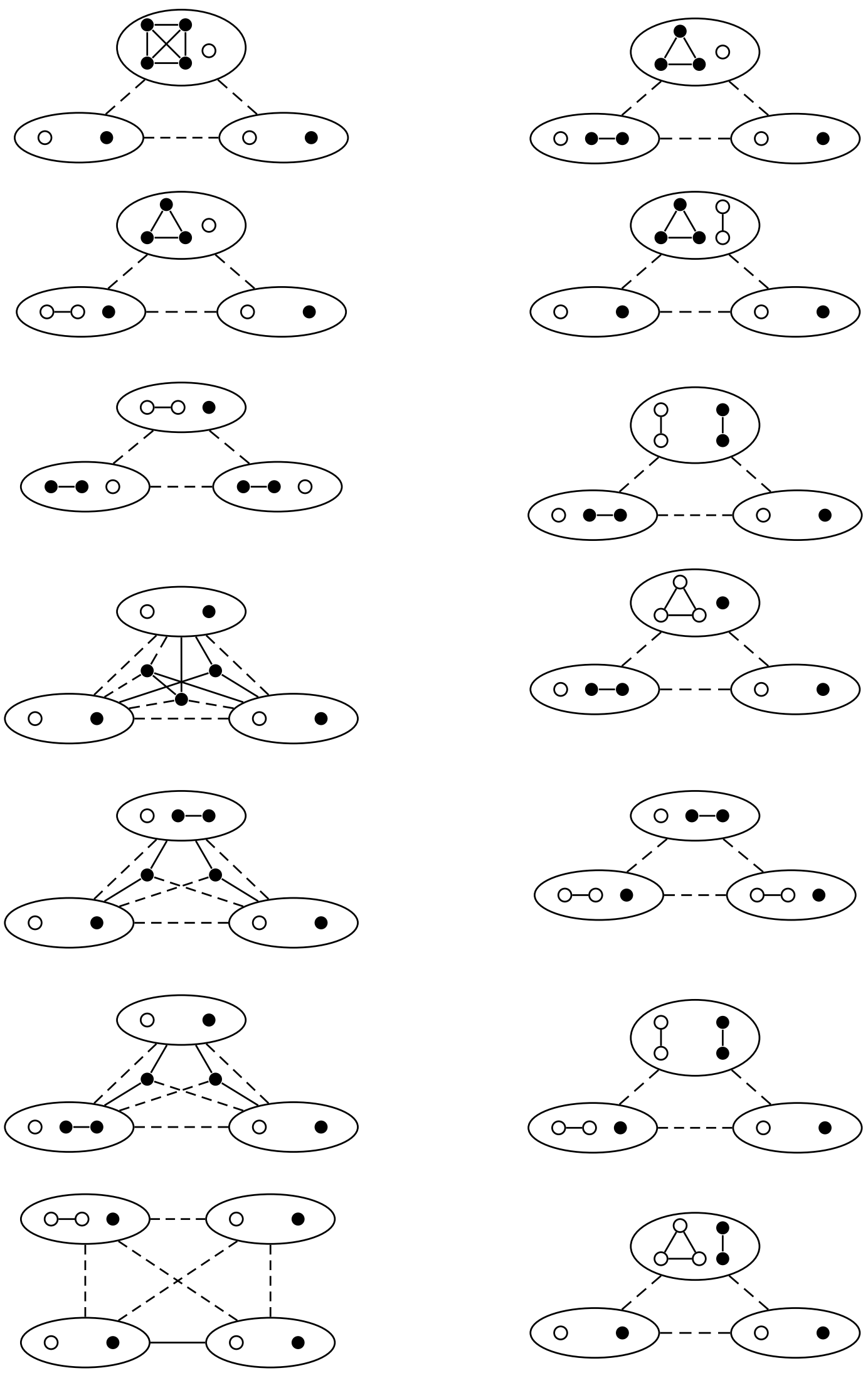

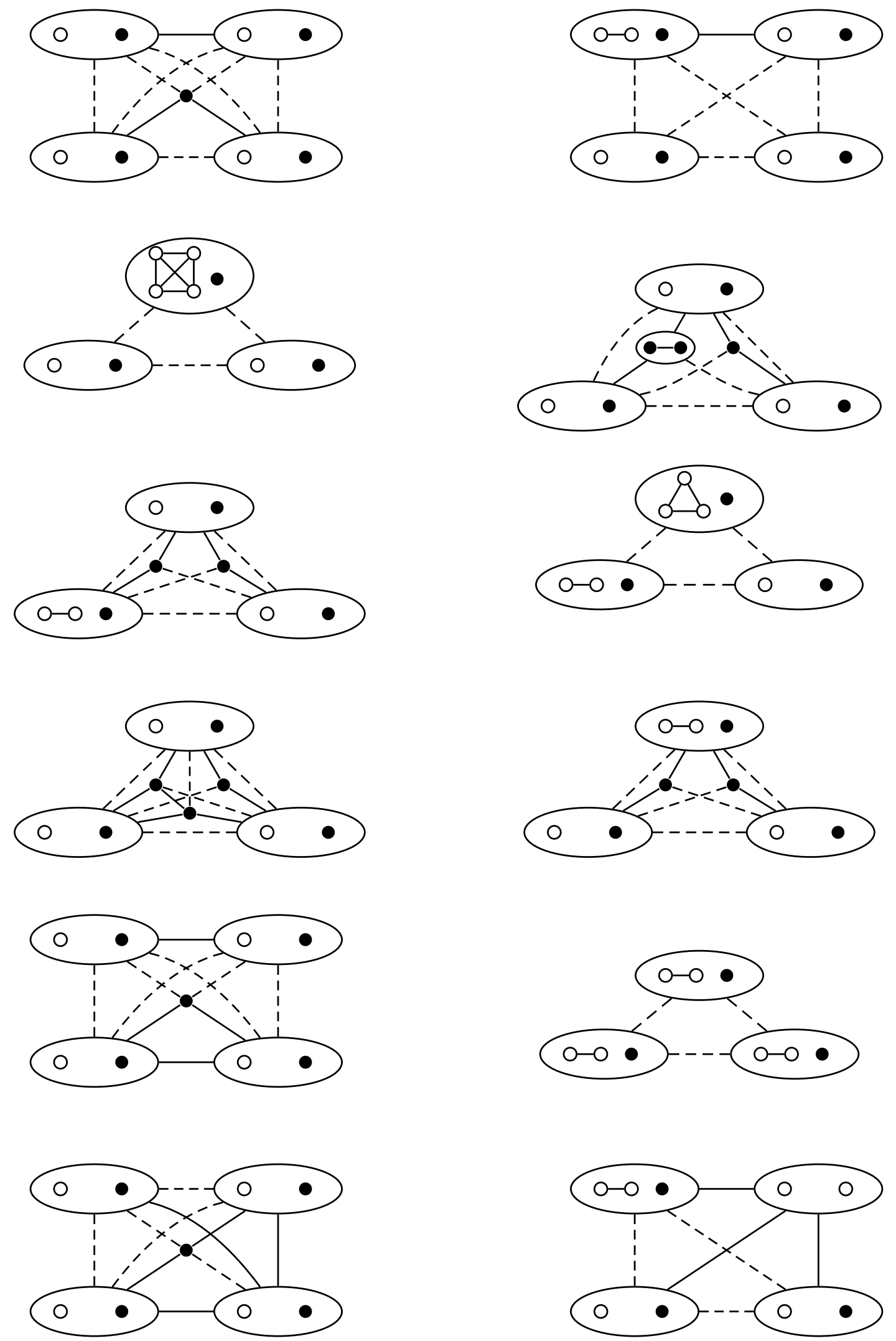

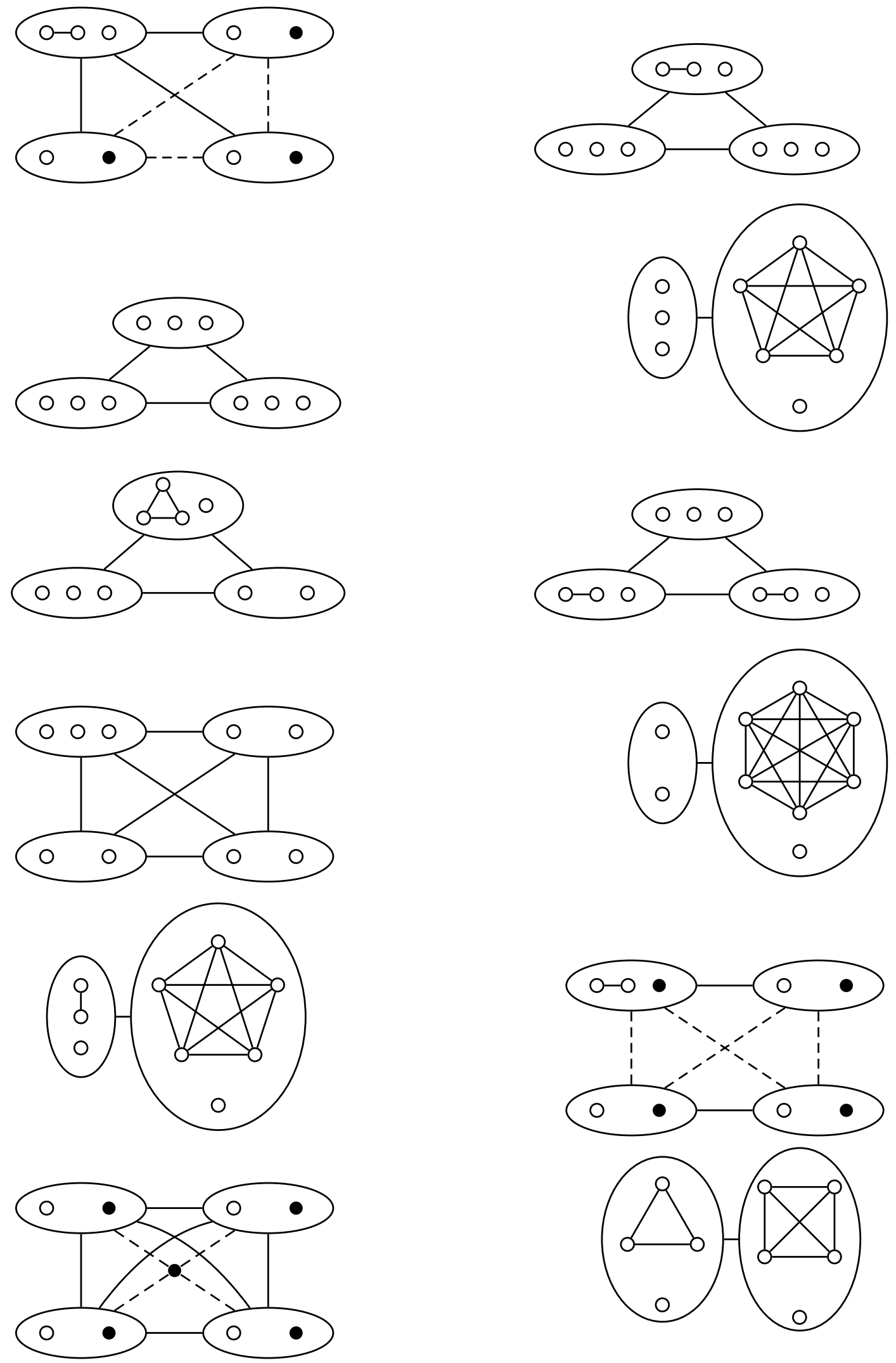
A.6. Relations on 9 elements
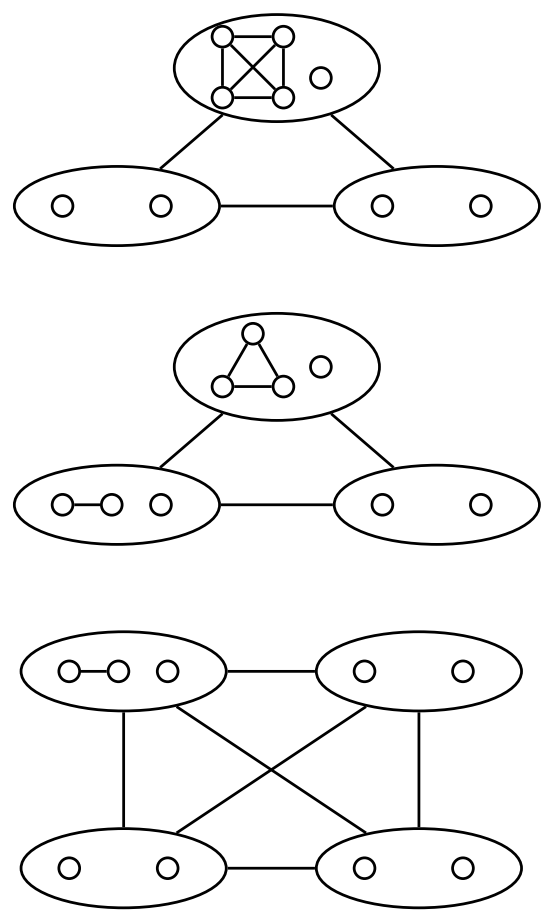
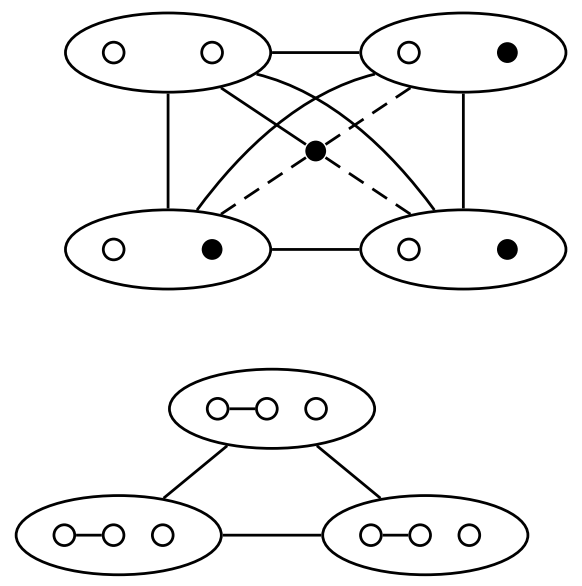

91 



\section{Appendix B.}

\section{Sažetak}

\section{Uvod}

Teorija klonova je moderna matematička disciplina koja proučava funkcionalne $\mathrm{i}$ relacione algebre. Ideje ugrađene $\mathrm{u}$ njene osnove dolaze iz nekoliko izvora. Jedan od njih je elementarna teorija 0/1 funkcija ([45]), koju je 1847. godine uveo Džordž Bul, predlažući da se iskazni veznici tretiraju kao diskretne istinitosne funkcije. Time je započeto izučavanje zatvorenih klasa istinitosnih funkcija u dvovrednosnoj i viševrednosnoj logici. Drugi važan izvor je klasična teorija Galoa. Inspirisana Erlangen programom Feliksa Klajna iz 1872. godine , ova teorija je evoluirala u opštu teoriju Galoa za funkcije i relacije. Pionirima ove oblasti se smatraju Mark Krasner([21, 47]) i Hoze Sebastiao e Silva([50, 48]), a njihova istraživanja se moraju sagledati u kontekstu napora matematike sa početka dvadesetog veka da uvede apstraktni koncept struktura, što je na kraju dovelo do razvoja opšte algebre i teorije modela. Teorija funkcionalnih algebri i opšta teorija Galoa za funkcije i relacije su se postepeno razvili u oblast koja je danas poznata kao teorija klonova(videti na pr. [19, 20],[15, 2, 52, 35]).

Osnovni problem teorije klonova je opis mreže klonova na datom skupu. Prvi značajniji rezultat $u$ ovoj oblasti objavio je Emil Leon Post $u$ [42, 43], gde je opisana mreža klonova na dvoelementnom skupu. To je ujedno i jedina do sada potpuno opisana mreža klonova, a već na troelementnom skupu ova mreža je uglavnom nepoznata i neistražena. Budući da se potpun opis mreže klonova u opštem slučaju smatra beznadežnim, istraživanja u ovoj oblasti se usredsređuju na izučavanje njenih podstruktura i aproksimacija.

Jedan od načina da se aproksimira mreža klonova jeste da se izučavaju $k$ ti delovi klonova i da se opišu njihove međusobne inkluzije. Stoga je i jedna od početnih tačaka ovog istraživanja bila izučavanje njihovih unarnih delova, odnosno, monoida transformacija. Prva klasa relacija, čiji monoidi endomorfizama i njihovi međusobni odnosi su izučavani, je bila klasa Rozenbergovih relacija ([36, 30, 34]). Ovakav izbor nije neočekivan, s obzirom da Rozenbergove relacije definišu maksimalne klonove. Ispostavilo se da su unarni delovi maksimalnih klonova skoro uvek neuporedivi, osim u nekoliko slučajeva (centralne, $h$-regularne relacije). U svakom slučaju, ad hoc metode koje su koršćene u ovoj studiji nisu bile dovoljne za dobijanje zadovoljavajućih rezultata u nekim od slučajeva u kojima se naslućivalo da inkluzije između monoida endomorfizama ipak postoje. Time se ukazala potreba za pronalaženjem novih metoda za rešavanje problema navedenog tipa, što je dovelo do razvoja lokalnih me- 
toda. One se pojavljuju prvi put u [37], gde su korišćene za izučavanje monoida endomorfizama centralnih relacija. Nakon toga, lokalne metode su uspešno primenjene na još nekoliko klasa relacija u [33]. U [29] je ukazano na iznenađujuću povezanost lokalnih metoda sa homomorfizam-homogenim relacijama. Svaki put kada radimo sa homomorfizam-homogenim relacijama možemo da koristimo lokalne metode, što je sugerisalo da postoji veza između homomorfizamhomogenosti i ciljeva ovog istraživanja.

Homomorfizam-homogenost je osobina relacionih struktura na koju su ukazali Piter Kameron i Jaroslav Nešetril u [5], kao na interesantno i prirodno uopštenje klasičnog pojma homogenosti. Problem utvrđivanja da li data relaciona struktura poseduje osobinu homogenosti ili ne je još uvek aktuelan, a do sada je dobijeno mnogo kombinatornih rezultata u ovoj oblasti $([14,24,49$, 23, 7, 3, 16]). Time je motivisano postavljanje i izučavanje sličnih pitanja u vezi sa homomorfizam homogenšću grafova i binarnih relacija, uopšte. U međuvremenu, pojavili su se i prvi rezultati na ovu temu. Homomorfizam-homogena stroga uređenja okarakterisali su Kameron i Debi Lokit u [4], dok je homomorfizam-homogena parcijalna uređenja u potpunosti opisao Dragan Mašulović u [28]. Nakon toga, pažnja je ponovo usmerena na grafove, što je dovelo do karakterizacije homomorfizamhomogenih konačnih turnira sa petljama u [18].

Klasična homogenost je polje velikog interesovanja zbog svoje povezanosti sa osobinom eliminacije kvantifikatora, zatim sa oligomorfnim grupama permutacija, itd. Štaviše, postoji veoma dobro razvijena teorija konstrukcija homogenih struktura, zahvaljujući radu Fraisea ([10, 11]). Sa druge strane, izučavanje homomorfizam-homogenih je, do sada, bio više kombinatorni problem rešavan iz čiste znatiželje.

Zahvaljujući do sada predstavljenim činjenicama, u mogućnosti smo da definišemo i ciljeve ove teze. Prvo, želimo da lokalne metode razvijemo u toj meri da ih je moguće koristiti u izučavanju unarnih delova klonova, uopšte. Drugo, cilj nam je da razvijemo vezu između homomomorfizam-homogenih struktura i lokalnih metoda. Konačno, naš treći cilj je razvoj sistematične teorije za klasifikaciju homomorfizam-homogenih struktura.

Teza počinje poglavljem posvećenim lokalnim metodama. U njemu je definisan Princip lokalnosti i uvedene su lokalne metode. U nastavku je pokazano kako se one mogu primeniti u izučavanju strukture slabih Krasnerovih algebri. Lokalne metode su razvijene za sve klase Rozenbergovih relacija. Na kraju poglavlja je dato nekoliko primera u kojima je demonstrirana primena ovih metoda.

U drugom poglavlju je sistematizovana upotreba Principa lokalnosti. Uveden je pojam $k$-endolokalnosti, $\mathrm{u}$ odnosu na koji su relacione strukture raspoređene $\mathrm{u}$ okviru hijerarhije. U sledećem koraku, $k$-endolokalne relacione strukture su izučavane sa tačke gledišta teorije modela. Klasični pojmovi teorije modela koji su korišćeni u sistematskoj studiji o homogenim strukturama su prilagođeni za izučavanje $k$-endolokalnih relacionih struktura. Na primer, pojmu $\omega$-kategoričnosti odgovara pojam slabe oligomorfnosti. Takođe, pojmu slabe homogenosti odgovara osobina proširenja za jednu tačku, dok eliminacionim skupovima od- 
govaraju pozitivni egzistencijalni eliminacioni skupovi. Ovo poglavlje kulminira sa Glavnom teoremom koja opisuje međusobne odnose između svih ovih pojmova. Time se uspostavlja veza između homomorfizam-homogenih relacionih struktura i pojmova iz teorije modela, čime se omogućava izučavanje homomorfizam-homogenih struktura korišćenjem model-teoretskih i algebarskih metoda. Iz Glavne teoreme proističu i važne posledice $u$ vezi sa teorijom klonova. Na primer, slabo oligomorfne slabe Krasnerove algebre na konačnom skupu su uvek lokalno zatvorene, čime se uopštava deo Glavne teoreme teorije klonova na slabo oligomorfne relacione strukture. Sa druge strane, naša Glavna teorema omogućava korišćenje lokalnih metoda na svim slabim Krasnerovim algebrama na konačnim skupovima.

U trećem poglavlju se teorija razvijena u drugom poglavlju koristi za razvoj sistematične metode za karakterizaciju homomorfizam-homogenih relacionih struktura. Ključni pojam u ovom delu teze je pojam minimalnih svedoka. U pitanju su specijalne podkonfiguracije koje su zabranjene u svim relacionim strukturama koje imaju osobinu proširenja za jednu tačku. Ako je za datu klasu relacionih struktura moguće pronaći sve minimalne svedoke do na izomorfizam, onda su time, na neki način, okarakterisane sve strukture iz date klase koje imaju osobinu proširenja za jednu tačku. Ovde su opisani svi minimalni svedoci do na izomofizam za klasu antisimetričnih tranzitivnih binarnih relacija, odnosno, za strukture oblika $(A, \varrho)$, gde je $\varrho$ binarna antisimetrična tranzitivna relacija. Ovi rezultati su zatim korišćeni za karakterizaciju svih tranzitivnih binarnih relacija sa osobinom proširenja za jednu tačku, čime su uopšteni rezultati Kamerona, Lokit i Mašulovića za stroga i parcijalna uređenja ([4, 28]). Takođe su na neki način opisani i minimalni svedoci za refleksivne simetrične binarne relacije, budući da se kod njih svaka konfiguracija može pojaviti kao potencijalni svedok. U svakom slučaju, ovakav ishod je bio očekivan, s obzirom da je $u$ [46] pokazano da je problem utvrđivanja homomorfizam-homogenosti za refleksivne simetrične binarne relacije co-NP kompletan. Ipak, homomorfizam-homogene refleksivne simetrične binarne relacije na konačnim skupovima su relativno retke. U Prilogu A su date, do na izomorfizam, sve netrivijalne homomorfizam-homogene refleksivne simetrične binarne relacije na skupu od najviše devet elemenata.

U nastavku dajemo detaljniji pregled poglavlja u kojima su izloženi originalni rezultati ove disertacije.

\section{Prvo poglavlje: Lokalne metode za relacione strukture}

Kao što smo već napomenuli, glavni cilj teorije klonova je proučavanje strukture mreže klonova. Jedan od mogućih pristupa ovom problemu je particija ove mreže u monoidalne intervale, odnosno, intervale nad unarnim delovima klonova. Ovim se problem izučavanja mreže klonova deli na izučavanje strukture monoidalnih intervala (kao na pr. $u$ [22]), sa jedne, i izučavanje međusobnih veza između ovih intervala, sa druge strane. Jedan od osnovnih problema koji 
se javlja u izučavanju međusobnih veza između ovih intervala je sledeći:

Neka su $\varrho$ i $\sigma$ relacije na istom skupu $A$.

$$
\text { Ispitati da li je End }\{\varrho\} \subseteq \text { End }\{\sigma\} \text {. }
$$

Sličan problem se javio u istraživanju čiji rezultati su objavljeni u [1] i [27], a koje je uključivalo i problem opisivanja strukture poseta tragova maksimalnih klonova. Rešavanje ovog problema se bazira na ispitivanju sadržanosti monoida endomorfizama jedne $u$ monoidu endomorfizama druge relacije, za svaki par Rozenbergovih relacija. Sličan problem su postavili i Pešel i Radelecki, a u vezi sa karakterizacijom svih kvazi-uređenja koja su invarijantna za datu unarnu algebru. Sve ovo je motivisalo nezavisno izučavanje problema (1).

Početni rezultati u ovoj oblasti su dobijeni u [30, 33, 34, 37, 38]. Međutim, metode koje su primenjivane su bile uglavnom ad hoc karaktera i zavisile su od konkretnih relacija sa kojima je rađeno. U ovom poglavlju je razvijen opšti pristup problemu, bez obzira na prirodu datih relacija. Ključna ideja je korišćenje tzv. lokalne metode koja se bazira na na pokazivanju da je potreban i dovoljan uslov za inkluziju između monoida endomorfizama dve relacije postojanje izvesnog načina kodiranja jedne relacije u drugu. Istraživanje je pokazalo da ova inkluzija uglavnom zavisi od relacije $\varrho$. Efikasnost procesa ispitivanja postojanja inkluzije zavisi od izbora načina kodiranja relacije $\varrho \mathrm{u}$ relaciju $\sigma$, a kodiranje koje mi koristimo zavisi samo od $\varrho$. Sledi formalan opis ove metode.

Princip lokalnosti. U pitanju je način definisanja sistema zatvorenja. U opštem slučaju, dat nam je prostor tačaka $A$ i uređen skup $\mathbf{T}=(\mathbb{T}, \sqsubseteq)$ mogućih opažanja koje nazivamo tipovima i koje može da prikupi posmatrač koji stoji u datoj tački. Ovakav posmatrač je ništa drugo do funkcija koja preslikava svaku tačku datog prostora u skup opažanja koje je moguće napraviti iz te tačke. Ovakvu funkciju zovemo tip-funkcija i označavamo je sa type ${ }_{\mathbf{A}}$.

Princip lokalnosti definiše na prostoru A skup implikacija na sledeći način:

Za tačke $\bar{a}$ and $\bar{b}$ implikacija $\bar{a} \rightarrow \bar{b}$ važi ako type $\mathbf{A}(\bar{a}) \sqsubseteq \operatorname{type}_{\mathbf{A}}(\bar{b})$.

Ovim je definisan sistem zatvorenja na prostoru A i za taj sistem kažemo da je definisan Principom lokalnosti.

Neka je data relacija $\varrho$ na konačnom skupu $A$. Naš cilj je da opišemo sistem zatvorenja (Inv End $\{\varrho\})^{(m)}$, za $m \in \mathbb{N}$ korišćenjem Principa lokalnosti. Za dati uređeni skup opažanja $\mathbf{T}=(\mathbb{T}, \sqsubseteq)$, funkcija type ${ }_{\rho}: A \rightarrow \mathbb{T}$ naziva se tip-funkcija za $\varrho$ i $m$ ako definiše sistem zatvorenja (Inv End $\{\varrho\})^{(m)}$ korišćenjem Principa lokalnosti.

Za svaku relaciju $\sigma \subseteq A^{m}$ važi End $\{\varrho\} \subseteq \operatorname{End}\{\sigma\}$ ako i samo ako za svako $\bar{a}, \bar{b} \in A^{m}$ ako $\bar{a} \in \sigma$ i type $_{\varrho}(\bar{a}) \sqsubseteq \operatorname{type}_{\varrho}(\bar{b})$, onda $\bar{b} \in \sigma$.

U nastavku poglavlja konstruisane su tip-funkcije za svih šest klasa Rozenbergovih relacija. 


\section{Drugo poglavlje: Endolokalne relacione strukture}

U prethodnom poglavlju sve tip-funkcije su dobijene ad hoc metodama, pa našu studiju ovde nastavljamo prelaskom na sistematičan pristup problemu.

Neka je data relaciona signatura $R$. Sa $\Phi(\exists, \wedge, \vee,=, R)$ označavamo skup svih pozitivnih egzistencijalnih formula nad $R$, dok sa $\Phi_{m}(\exists, \wedge, \vee,=, R)$ označavamo skup svih pozitivnih egzistencijalnih formula nad $R$ čije su slobodne promenljive sadržane u skupu $\left\{x_{1}, \ldots, x_{m}\right\}$. Slično tome, sa $\Phi^{(k)}(\exists, \wedge, \vee,=, R)$ onačavamo skup svih pozitivnih egzistencijalnih formula čija dubina kvantifikacije je najviše k. Dalje, definišemo kontekste $\mathbb{K}_{m}(\mathbf{A}):=\left(A^{m}, \Phi_{m}(\exists, \wedge, \vee,=, R), \models\right)$ i $\mathbb{K}_{m}^{(k)}(\mathbf{A}):=$ $\left(A^{m}, \Phi_{m}^{(k)}(\exists, \wedge, \vee,=, R), \models\right)$, gde je $\models$ relacija definisana na sledeći način:

Definicija. Kažemo da $m$-torka $\bar{a}$ zadovoljava formulu $\varphi$ i pišemo $\bar{a} \vDash \varphi$ ako postoji valuacija $v$ takva da $v\left(x_{i}\right)=a_{i}$, za sve $i \in\{1, \ldots, m\}$ i $\mathbf{A} \vDash_{v} \varphi$.

Navedeni kontekst definiše Galoa vezu između skupova $m$-torki i skupova formula. Galoa zatvoreni skupovi $m$-torki nazivaju se ekstenti, a Galoa zatvoreni skupovi formula intenti. Galoa operator za intente je takođe označen sa $\mathrm{pTh}_{\mathrm{A}}$.

Definicija. Kažemo da je relaciona struktura A slabo oligomorfna ako za svako $m \in \mathbb{N}$ kontekst $\mathbb{K}_{m}(\mathbf{A})$ ima samo konačno mnogo različitih ekstenata.

Definicija. Relaciona struktura $\mathbf{A}=\left(A,\left(\varrho_{\mathbf{A}}\right)_{\varrho \in R}\right)$ naziva se $k$-endolokalnom ako za svako $m \in \mathbb{N}$ i svaku $\sigma \subseteq A^{m}$ važi

$$
\sigma=\sigma^{\prime \prime} \text { ako i samo ako } \forall \bar{a}, \bar{b} \in A^{m}: \bar{a} \in \sigma \wedge \bar{b} \vDash \operatorname{pTh}_{\mathrm{A}}^{(k)}(\bar{a}) \Rightarrow \bar{b} \in \sigma .
$$

Ako je relaciona struktura 0-endolokalna, onda je nazivamo samo endolokalna.

Definicija. Neka je $\mathbf{A}=\left(A,\left(\varrho_{\mathbf{A}}\right)_{\varrho \in R}\right)$ relaciona struktura. Skup $\Psi \subseteq \Phi(\exists, \wedge, \vee,=, R)$ se naziva pozitivni egzistencijalni eliminacioni skup za $\mathbf{A}$ ako za svaku formulu $\varphi\left(x_{1}, \ldots, x_{m}\right) \in \Phi(\exists, \wedge, \vee,=, R)$ postoji formula $\varphi^{*}\left(x_{1}, \ldots, x_{m}\right) \in \Phi(\exists, \wedge, \vee,=, R)$ koja je disjunkcija konjukcija formula iz $\Psi$ i $\varphi$ je ekvivalentno sa $\varphi^{*}$ u A.

Definicija. Kažemo da relaciona struktura A ima osobinu proširenja za jednu tačku ako za svaku konačnu podstrukturu $\mathbf{B}$ od $\mathbf{A}$, svako $b \in A \backslash B$ i svaki homomorfizam $f: \mathbf{B} \rightarrow \mathbf{A}$, postoji homomorfizam $g: \mathbf{B} \cup\{b\} \rightarrow \mathbf{A}$ koji proširuje $f$.

Definicija. Data je relaciona struktura $\mathbf{A}=\left(A,\left(\varrho_{\mathbf{A}}\right)_{\varrho \in R}\right)$ i njena konačna podstruktura $\mathbf{B}=\left(B,\left(\varrho_{\mathbf{B}}\right)_{\varrho \in R}\right)$. Kažemo da je $c \in A$ slabi centar za $\mathbf{B}$ ako

za svako $b \in B$ postoji $\varrho \in R, b_{3}, \ldots, b_{\operatorname{ar}(\varrho)} \in B$ i $\pi \in \operatorname{Sym}\{1, \ldots, \operatorname{ar}(\varrho)\}$ takvi da

$$
\left(c, b, b_{3}, \ldots, b_{\operatorname{ar}(\varrho)}\right)^{\pi} \in \varrho_{\mathbf{A}} .
$$

Definicija. Kažemo da relaciona struktura $\mathbf{A}=\left(A,\left(\varrho_{A}\right)_{\varrho \in R}\right)$ ima osobinu slabog proširenja za jednu tačku ako za svaku konačnu podstrukturu B od A, svaki homomorfizam $f: \mathbf{B} \rightarrow \mathbf{A}$ i svaki slabi centar $c$ od $\mathbf{B}$ postoji homomorfizam $g: \mathbf{B} \cup\{c\} \rightarrow \mathbf{A}$ takav da $g \uparrow_{B}=f$. 
Definicija. Strukturu D zovemo homomorfizam-homogenom ako se svaki lokalni homomorfizam od D može proširiti do endomorfizma od D.

Glavna teorema daje vezu između svih gore uvedenih pojmova:

Teorema. Neka je $\mathbf{A}=\left(A,\left(\varrho_{\mathbf{A}}\right)_{\varrho \in R}\right)$ relaciona struktura. Tada važe relacije date $u$ sledećem dijagramu:

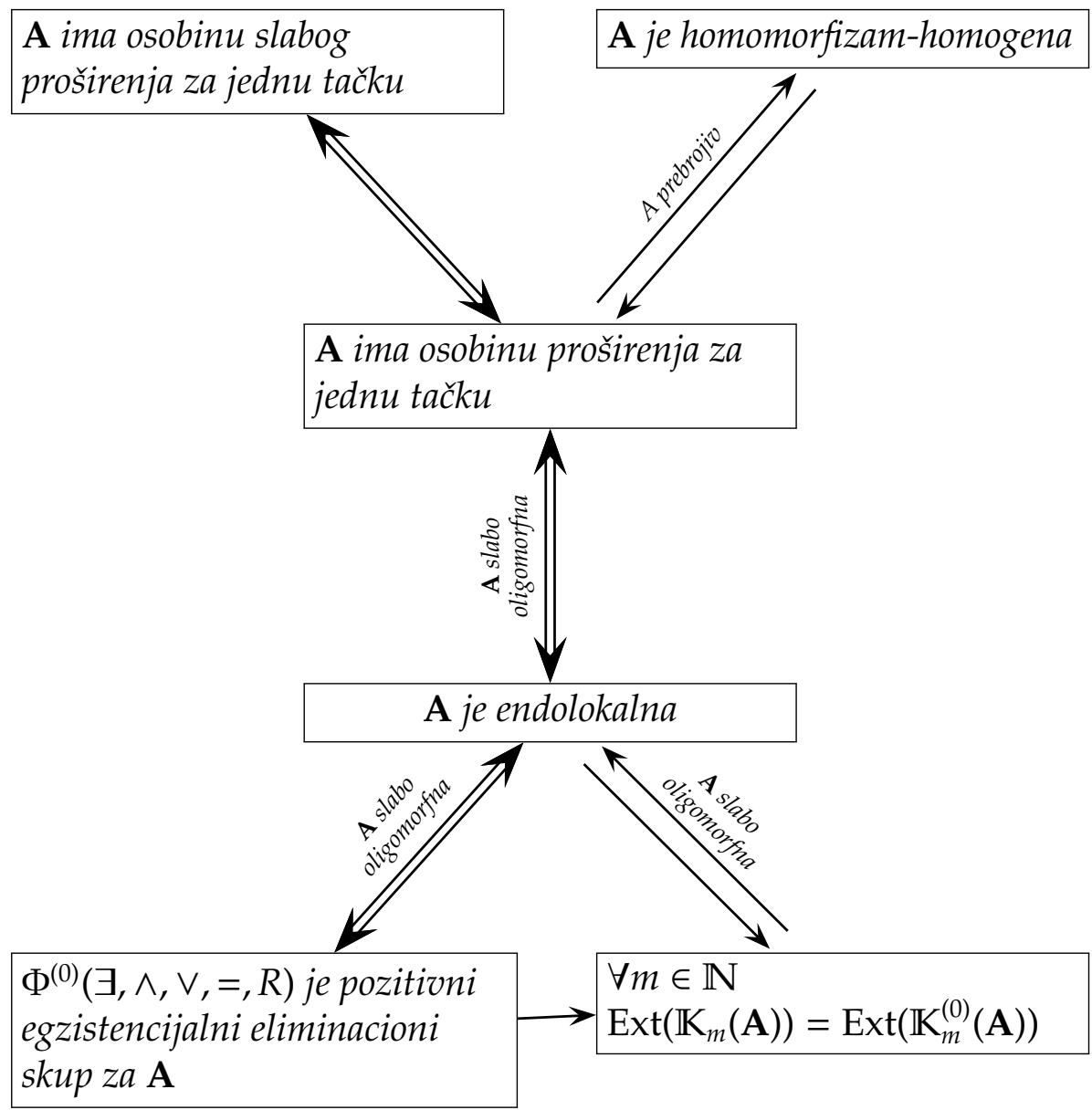

Gore razvijena teorija nam omogućava da izvedemo sledeće interesantne posledice za teoriju klonova:

1. Ako je data endolokalna struktura sa konačnom signaturom, onda se $m$-arni deo njene slabe Krasnerove algebre može efektivno opisati.

2. Ako je data slabo oligomorfna homomorfizam-homogena relaciona struktura $\mathbf{A}=\left(A,\left(\varrho_{A}\right)_{\varrho \in R}\right)$, onda je slaba Krasnerova algebra definisana ovom strukturom lokalno zatvorena, tj.

$$
\operatorname{Inv} \operatorname{End}(\mathbf{A})=\left[\left(\varrho_{\mathbf{A}}\right)_{\varrho \in R}\right]_{\text {WKA }} \text {. }
$$

3. Za konačnu endolokalnu relacionu strukturu A sa konačnom signaturom može se lako testirati da li je data relacija sadržana u slaboj Krasnerovoj algebri opisanoj sa $\mathbf{A}$. 


\section{Treće poglavlje: Homomorfizam-homogene relacione strukture}

Ovo poglavlje je posvećeno sistematskoj studiji homomorfizam-homogenih relacionih struktura. Korišćenjem rezultata sakupljenih u Glavnoj teoremi, izvodimo kriterijum za karakterizaciju relacionih struktura ove vrste. Spomenuti kriterijum nazivamo kriterijum minimalnog svedoka i on je baza za metod eliminacije svih relacionih struktura koje nisu homomorfizam-homogene, a nalaze se $u$ datoj klasi relacionih struktura.

Definicija. Neka je A relaciona struktura. Svedokom nazivamo uređenu četvor$\mathrm{ku}\left(\mathbf{B}_{1}, \mathbf{B}_{2}, f, c\right)$, gde je $\mathbf{B}_{1}$ konačna podstruktura od $\mathbf{A}, c$ je slabi centar za $\mathbf{B}_{\mathbf{1}} \mathbf{u} \mathbf{A}$, $\mathbf{B}_{2}$ je podstruktura od $\mathbf{A}$, i $f: \mathbf{B}_{1} \rightarrow \mathbf{B}_{2}$ je surjekcija, koja ne može da se proširi do $\mathbf{B}_{1} \cup\{c\}$.

Na skupu svih svedoka date relacione strukture može se definisati kvaziuređenje tako da za svaka dva uporediva svedoka, postojanje većeg implicira postojanje manjeg. Minimalni svedok je minimalni element $\mathrm{u}$ ovom kvaziuređenju. Ako je data klasa relacionih struktura za koju želimo da pronađemo sve homomorfizam-homogene relacione strukture, onda to možemo učiniti tako što ćemo do na izomorfizam opisati sve moguće minimalne svedoke. Struktura koja ne sadrži nijedan od minimalnih svedoka je homomorfizam-homogena. Ovaj kriterijum nazivamo kriterijumom minimalnog svedoka.

Za klasu relacionih struktura $(A, \varrho)$, gde je $A$ proizvoljan skup, a $\varrho$ antisimetrična tranzitivna relacija, izlistani su svi mogući minimalni svedoci do na izomorfizam (videti od strane 69). Korišćenjem ovih rezultata okarakterisane su homomorfizam-homogene tranzitivne relacije i, specijalno, homomorfizamhomogena kvazi-uređenja.

U nastavku su opisani minimalni svedoci za refleksivne simetrične relacije i data je metoda za konstruktivno prebrojavanje ovakvih homomorfizam-homogenih relacija na malim konačnim skupovima. U Prilogu A su date do na izomorfizam sve netrivijalne homomorfizam-homogene refleksivne simetrične relacije na skupu do 9 elemenata. 



\section{Bibliography}

[1] E. Aichinger, D. Mašulović, R. Pöschel, and J. S. Wilson. Completeness for concrete near-rings. J. Algebra, 279(1):61-78, 2004.

[2] V. G. Bodnarčuk, L. A. Kalužnin, V. N. Kotov, and B. A. Romov. Galois theory for Post algebras. I, II. Kibernetika (Kiev), (3):1-10; ibid. 1969, no. 5, $1-9,1969$.

[3] P. J. Cameron. Homogeneous permutations. Electron. J. Combin., 9(2):Research paper 2, 9 pp. (electronic), 2002/03. Permutation patterns (Otago, 2003).

[4] P. J. Cameron and D. Lockett. Posets, homomorphisms and homogeneity. to appear in Disc. Math.

[5] P. J. Cameron and J. Nešetřil. Homomorphism-homogeneous relational structures. Combin. Probab. Comput., 15(1-2):91-103, 2006.

[6] C. C. Chang and H. J. Keisler. Model theory, volume 73 of Studies in Logic and the Foundations of Mathematics. North-Holland Publishing Co., Amsterdam, third edition, 1990.

[7] G. L. Cherlin. The classification of countable homogeneous directed graphs and countable homogeneous n-tournaments. Mem. Amer. Math. Soc., 131(621):xiv+161, 1998.

[8] B. A. Davey and H. A. Priestley. Introduction to lattices and order. Cambridge Mathematical Textbooks. Cambridge University Press, Cambridge, 1990.

[9] K. Denecke, M. Erné, and S. L. Wismath, editors. Galois connections and applications, volume 565 of Mathematics and its Applications. Kluwer Academic Publishers, Dordrecht, 2004.

[10] R. Fraïssé. Sur certaines relations qui généralisent l'ordre des nombres rationnels. C. R. Acad. Sci. Paris, 237:540-542, 1953.

[11] R. Fraïssé. Theory of relations, volume 145 of Studies in Logic and the Foundations of Mathematics. North-Holland Publishing Co., Amsterdam, revised edition, 2000. With an appendix by Norbert Sauer.

[12] B. Ganter and R. Wille. Formal concept analysis. Springer-Verlag, Berlin, 1999. Mathematical foundations, Translated from the 1996 German original by Cornelia Franzke. 
[13] The GAP Group. GAP - Groups, Algorithms, and Programming, Version 4.4.10, 2007.

[14] A. Gardiner. Homogeneous graphs. J. Combinatorial Theory Ser. B, 20(1):94102, 1976.

[15] D. Geiger. Closed systems of functions and predicates. Pacific J. Math., 27:95-100, 1968.

[16] J. J. Gol'fand and M. H. Klin. On k-homogeneous graphs. In Algorithmic studies in combinatorics (Russian), pages 76-85, 186 (errata insert). "Nauka", Moscow, 1978.

[17] W. Hodges. A shorter model theory. Cambridge University Press, Cambridge, 1997.

[18] A. Ilić, D. Mašulović, and U. Rajković. Finite homomorphism-homogeneous tournaments with loops. J. Graph Theory, 59(1):45-58, 2008.

[19] S. V. Jablonskiǔ. Functional constructions in a $k$-valued logic. Trudy Mat. Inst. Steklov., 51:5-142, 1958.

[20] S. V. Jablonskiı̌, G. P. Gavrilov, and V. B. Kudrjavcev. Funktsii algebry logiki i klassy Posta. Izdat. "Nauka”, Moscow, 1966.

[21] M. Krasner. Une généralisation de la notion de corps. J. Math. pure et appl. (Liouville Journal), 17:367-385, 1938.

[22] A. A. Krokhin. Monoidal intervals in lattices of clones. Algebra i Logika, 34(3):288-310, 364, 1995.

[23] A. H. Lachlan. Countable homogeneous tournaments. Trans. Amer. Math. Soc., 284(2):431-461, 1984.

[24] A. H. Lachlan and R. E. Woodrow. Countable ultrahomogeneous undirected graphs. Trans. Amer. Math. Soc., 262(1):51-94, 1980.

[25] D. Lau. Bestimmung der Ordnung maximaler Klassen von Funktionen der k-wertigen Logik. Z. Math. Logik Grundlag. Math., 24(1):79-96, 1978.

[26] D. Lau. Function algebras on finite sets. Springer Monographs in Mathematics. Springer-Verlag, Berlin, 2006. A basic course on many-valued logic and clone theory.

[27] D. Mašulović. On a completeness criterion for 0-symmetric near-rings. preprint MATH-AL-17-2002, TU Dresden, 2002.

[28] D. Mašulović. Homomorphism-homogeneous partially ordered sets. Order, 24(4):215-226, 2007. 
[29] D. Mašulović. On endomorphism monoids of partial orders and central relations. Novi Sad J. Math., 38(1):111-125, 2008.

[30] D. Mašulović and M. Pech. On traces of maximal clones. Novi Sad J. Math., 35(1):161-185, 2005.

[31] B. D. McKay. nauty User's Guide (Version 2.4). Department of Computer Science, Australian National University, Canberra, 2007.

[32] M. Pech. Local methods for Rosenberg relations. to appear in Algebra Universalis.

[33] M. Pech. On local methods for traces of maximal clones. preprint MATHAL-02-2005, TU Dresden, 2005.

[34] M. Pech and D. Mašulović. On the height of the poset of endomorphism monoids of regular relations. Journal of Multiple-Valued Logic and Soft Computing, 15(1):81-94, 2009.

[35] B. Poizat. Théorie de Galois pour les algèbres de Post infinitaires. Z. Math. Logik Grundlag. Math., 27(1):31-44, 1981.

[36] M. Ponjavić. On traces of maximal clones. Master's thesis, University of Novi Sad, 2003.

[37] M. Ponjavić. On the structure of the poset of endomorphism monoids of central relations. In Contributions to general algebra. 16, pages 189-197. Heyn, Klagenfurt, 2005.

[38] M. Ponjavić and D. Mašulović. On chains and antichains in the partially ordered set of traces of maximal clones. In Contributions to general algebra. 15, pages 119-134. Heyn, Klagenfurt, 2004.

[39] R. Pöschel. Galois connections for operations and relations. In Galois connections and applications, volume 565 of Math. Appl., pages 231-258. Kluwer Acad. Publ., Dordrecht, 2004.

[40] R. Pöschel and L. A. Kalužnin. Funktionen- und Relationenalgebren, volume 15 of Mathematische Monographien [Mathematical Monographs]. VEB Deutscher Verlag der Wissenschaften, Berlin, 1979. Ein Kapitel der diskreten Mathematik. [A chapter in discrete mathematics].

[41] R. Pöschel and S. Radeleczki. Private communication.

[42] E. L. Post. Introduction to a General Theory of Elementary Propositions. Amer. J. Math., 43(3):163-185, 1921. 
[43] E. L. Post. The Two-Valued Iterative Systems of Mathematical Logic. Annals of Mathematics Studies, no. 5. Princeton University Press, Princeton, N. J., 1941.

[44] I. Rosenberg. Über die funktionale Vollständigkeit in den mehrwertigen Logiken. Struktur der Funktionen von mehreren Veränderlichen auf endlichen Mengen. Rozpravy Československé Akademie Věd Ǩada Matematických a Př́rodních Věd, 80(4):93, 1970.

[45] I. Rosenberg. Clones of Boolean functions. Rapports de recherche du départment de mathématiques et de statistique, Université de Montréal, 1988.

[46] M. Rusinov and P. Schweitzer. Homomorphism-homogeneous graphs. submitted for publication, 2008.

[47] A. Schleiermacher. Über einen Satz von Krasner. I. Österreich. Akad. Wiss. Math.-Natur. Kl. Sitzungsber. II, 205:111-140 (1997), 1996.

[48] A. Schleiermacher. Über einen Satz von Krasner. II. Österreich. Akad. Wiss. Math.-Natur. Kl. Sitzungsber. II, 206:47-116 (1998), 1997.

[49] J. H. Schmerl. Countable homogeneous partially ordered sets. Algebra Universalis, 9(3):317-321, 1979.

[50] J. Sebastião e Silva. Para uma teoria geral dos homomorфsmos. In Obras de José Sebastião e Silva, volume Vol. I., pages 135-335. Instituto Nacional de Investigacao Cientifica, Lissabon, 1985.

[51] L. H. Soicher. The GRAPE package for GAP, Version 4.3, 2006.

[52] L. Szabó. Concrete representation of related structures of universal algebras. I. Acta Sci. Math. (Szeged), 40(1-2):175-184, 1978.

[53] Á. Szendrei. Clones in universal algebra, volume 99 of Séminaire de Mathématiques Supérieures [Seminar on Higher Mathematics]. Presses de l’Université de Montréal, Montreal, QC, 1986. 


\section{Biografija}

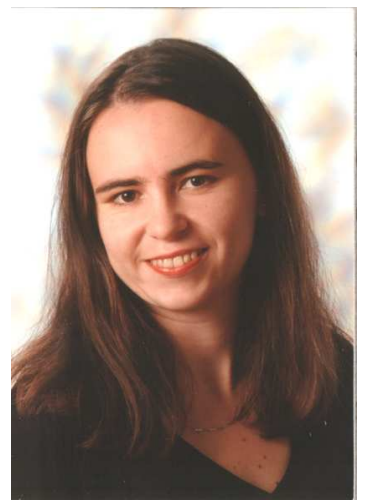

Rođena sam 8.maja 1977.godine u Novom Sadu. Nakon osnovne škole, koju sam pohađala u Futogu, nastavila sam školovanje na specijalnom matematičkom usmerenju u gimnaziji "Jovan Jovanović Zmaj"u Novom Sadu. Studije matematike na Prirodno-matematičkom fakultetu Univerziteta u Novom Sadu sam upisala 1996.godine i uspešno ih okončala 2000.godine sa prosečnom ocenom 9,68. Iste godine sam upisala postdiplomske studije iz oblasti Diskretne matematike. Položila sam sve ispite sa prosečnom ocenom 10,00. Zvanje magistra matematičkih nauka sam stekla 18.septembra 2003.godine, odbranom teze "Tragovi maksimalnih klonova".

Od marta 2001.godine sam zaposlena kao asistent-pripravnik, a od oktobra 2005.godine kao asistent na Departmanu za matematiku i informatiku Univerziteta u Novom Sadu. Boravila sam više puta na Institutu za algebru Univerziteta za tehnologije u Drezdenu, Nemačka, između ostalih dva puta kao stipendista DAAD-a (školske 2002/2003 i 2005/2006 godine) i dva puta kao stipendista DFG-a (u okvuru programa GK334, novembra 2003. i u letnjem semestru 2005.godine). U leto 2008.godine sam boravila po pozivu na Departmanu za matematiku Univerziteta "Ben Gurion"u Be'er Shevi, Izrael. Učestvovala sam na više naučnih konferencija u zemlji i inostranstvu, gde sam imala nekoliko izlaganja. Oblasti mog naučnog interesovanja su teorija klonova, diskretna matematika i teorija modela. Autor ili koautor sam pet naučnih radova .

Novi Sad, mart 2009.godine

Maja Pech

\section{Curriculum vitae}

I was born on May, $8^{\text {th }} 1977$ in Novi Sad. After the primary school, that I attended in Futog, I continued with my education at the secondary school "Jovan Jovanović Zmaj", Novi Sad where I enrolled in the special mathematical profile. In year 1996 I entered the studies of mathematics at the Faculty of Science and Mathematics of the University of Novi Sad and finished them successfully in 2000 with the average mark 9.68. Postgraduate studies in Discrete Mathematics I entered in in the same year and passed all the exams with the average mark 10.00. The title of Master of Science I received on September, $18^{\text {th }}$ 2003, after I defended the thesis "Traces of maximal clones".

Since March 2001 I was have been working as junior teaching assistant and since October 2005 as teaching assistant at the Department of Mathematics and Informatics, University of Novi Sad. I visited on several occasions the Institut of algebra, TU Dresden, Germany. Among others, I two these visits were supported by DAAD (2002/2003 and 2005/2006) and two visits were supported by DFG 
(November 2003 and summer semester 2005). In the summer 2008 I had an invited stay at the Department of mathematics, Ben Gurion University, Be'er Sheva, Izrael. I attended a number of scientific conferences at home and abroad, were I gave several talks. My scientific interests are in clone theory, discrete mathematics and model theory. I am the author and coauthor of five scientific papers.

Novi Sad, March 2009

Maja Pech 


\section{UNIVERZITET U NOVOM SADU \\ PRIRODNO-MATEMATIČKI FAKULTET \\ KLJUČNA DOKUMENTACIJSKA INFORMACIJA}

\section{Redni broj:}

RBR

\section{Identifikacioni broj:}

IBR

Tip dokumentacije: Monografska dokumentacija

TD

Tip zapisa: Tekstualni štampani materijal

\section{TZ}

Vrsta rada: Doktorska disertacija

VR

Autor: mr Maja Pech (rođena Ponjavić)

AU

Mentor: dr Dragan Mašulović, vanredni profesor

MN

Naslov rada: Local methods for relational structures and their weak Krasner algebras (Lokalne metode za relacione strukture i njihove slabe Krasnerove algebre)

MR

Jezik publikacije: engleski

JP

Jezik izvoda: engleski/srpski

JI

Zemlja publikovanja: Republika Srbija

ZP

Uže geografsko područje: Vojvodina

UGP 
Godina: 2009.

GO

Izdavač: Autorski reprint

IZ

Mesto i adresa: Novi Sad, Prirodno-matematički fakultet, Trg D. Obradovića 4 MA

Fizički opis rada:3/xviii+106/53/6/166/20/2

(broj poglavlja/strana/lit. citata/tabela/slika/dijagrama/priloga)

FO

Naučna oblast: Matematika

NO

Naučna disciplina: Diskretna matematika

ND

Ključne reči: lokalne metode, relacione strukture, teorija klonova, veze Galoa, monoidi endomorfizama, slabe Krasnerove algebre, homomorfizam-homogenost, Rozenbergove relacije, endolokalnost, proširenje za jednu tačku.

PO

UDK:

Čuva se: u biblioteci Departmana za matematiku i informatiku, Novi Sad ČU,

Važna napomena: Sažetak teze na srpskom jeziku dostupan je u Prilogu B.

VN

Izvod: U ovoj tezi su razvijene lokalne metode koje se mogu koristiti za izučavanje unarnih delova klonova (ili, ekvivalentno, slabih Krasnerovih algebri). Korišćenjem jezika teorije modela i Galoovih veza uspostavljen je odnos između homomorfizam-homogenih relacionih struktura i lokalnih metoda, preko pojma endolokalnosti. Dobijeni teoretski rezultati su upotrebljeni za razvoj sistematske teorije za klasifikaciju homomorfizam-homogenih struktura.

IZ

Datum prihvatanja teme od strane NN veća: 15.01 .2009 . 
DP

Datum odbrane:

DO

Članovi komisije:

KO

Predsednik: dr Igor Dolinka, redovni profesor PMF u Novom Sadu

Mentor: dr Dragan Mašulović, vanredni profesor PMF u Novom Sadu

Član: dr Ratko Tošić, redovni profesor Fakulteta tehničkih nauka u Novom Pazaru

Član: dr Ivica Bošnjak, docent PMF u Novom Sadu

Član: dr Reinhard Pöschel, redovni profesor Univerziteta za tehnologiju u Drezdenu, Nemačka 


\section{UNIVERSITY OF NOVI SAD \\ FACULTY OF SCIENCE \\ KEY WORDS DOCUMENTATION}

Accession number:

ANO

Identification number:

INO

Document type: Monograph type

DT

Type of record: Printed text

TR

Contents Code: PhD thesis

CC

Author: Maja Pech (née Ponjavić), MSc

AU

Mentor: dr Dragan Mašulović, associate professor

MN

Title: Local methods for relational structures and their weak Krasner algebras

XI

Language of text: English

LT

Language of abstract: English/Serbian

LA

Country of publication: Republic of Serbia

$\mathrm{CP}$

Locality of publication: Vojvodina

LP

Publication year: 2009 
Publisher: Author's reprint

PU

Publ. place: Novi Sad, Faculty of Science and Mathematics, Trg D. Obradovića 4

PP

Physical description:3/xviii+106/53/6/166/20/2

(chapters/pages/literature/tables/figures/diagrams/appendices)

PD

Scientific field: Mathematics

SF

Scientific discipline: Discrete mathematics

SD

Key words:local methods, relational structure, clone theory, Galois connections, endomorphism monoids, weak Krasner algebras, homomorphism-homogeneity, Rosenberg relations, endolocality, one-point extension.

UC:

Holding data: Library of the Department of Mathematics and Informatics, Novi Sad

HD:

Important note:

IN:

Abstract: In this thesis local methods are made available as a tool to study the unary parts of clones (or, equivalently, the weak Krasner algebras). Using the language of model theory and Galois connections we develop a link between homomorphism-homogeneous relational structures and local methods, via the notion of endolocality. The theoretical results that are obtained are used to develop a systematic theory for the classification of homomorphism-homogeneous relational structures.

AB

Accepted by the Scientific Board on: Jan, 15 2009 


\section{Defended:}

Thesis defence board:

Chair: dr Igor Dolinka, full professor, Faculty of Science, Novi Sad

Mentor: dr Dragan Mašulović, associate professor, Faculty of Science, Novi Sad Member: dr Ratko Tošić, full professor, Faculty of Technical sciences, Novi Pazar

Member: dr Ivica Bošnjak, assistant professor, Faculty of Science, Novi Sad Member: dr Reinhard Pöschel, full professor, TU Dresden, Germany 